\title{
7II
}

\section{Design and realization of novel GaAs based laser concepts}

\author{
vorgelegt von \\ Diplom-Physiker \\ Tim David Germann \\ aus Berlin
Von der Fakultät II - Mathematik und Naturwissenschaften der Technischen Universität Berlin zur Erlangung des akademischen Grades
Doktor der Naturwissenschaften
- Dr. rer. nat. -
genehmigte Dissertation

Promotionsausschuss:

Vorsitzender: Prof. Dr. Michael Lehmann

Berichter/Gutachter: Prof. Dr. Dieter Bimberg

Berichter/Gutachter: Prof. Shun Lien Chuang, PhD

Tag der wissenschaftlichen Aussprache: 21.12.2011

Berlin 2012

D 83 

Meinem geliebten Sohn Emil Malo 


\section{Zusammenfassung}

Halbleiterlaser stellen die Grundlage für eine zunehmende Vielzahl von Anwendungen dar, die von der Informationsspeicherung und digitalen Kommunikation bis hin zur Materialbearbeitung reichen. Neuartige Konzepte überwinden bisherige Limitierungen und erschließen neue Anwendungsgebiete. Viele dieser Anwendungsgebiete verlangen nach kostengünstigen Bauelementen, die maximale Brillanz und hohe Ausgangsleistungen oder höchste Geschwindigkeiten erreichen.

Diese Arbeit stellt dar, wie essentielle Leistungsmerkmale von Halbleiterlasern durch das Design von Nanostrukturen und epitaktischen Wachstumsprozessen maßgeschneidert werden können. Hierbei wird auf alle Schritte der Laserherstellung eingegangen, vom Design über das Wachstum der Nanostrukturen mittels metallorganischer Gasphasenepitaxie (MOVPE), bis hin zur Herstellung und Charakterisierung kompletter Bauelemente. Durch die Nutzung industrieller Standards können alle entwickelten Prozesse auch auf die Massenproduktion übertragen werden.

Gepulster Hochleistungslaserbetrieb bis zu $8 \mathrm{~W}$ und eine extrem niedrige Schwellstromdichte von nur $66 \mathrm{~A} / \mathrm{cm}^{2}$ wird mit Quantenpunkt (QP)-basierten Kantenemittern bei $1.25 \mu \mathrm{m}$ durch das verbesserte Verständnis des QP-Wachstumsprozesses erreicht. Dieser neue Wachstumsprozess ermöglicht Kantenemitter bei $1.3 \mu \mathrm{m}$ für Telekommunikationsanwendungen auf Basis des etablierten InGaAs/GaAs Materialsystems. Im Zentrum dieses Fortschritts steht die detaillierte Untersuchung und Optimierung von nahezu allen Einzelschichten der Laserstruktur. Verschiedene Wellenleiterdesigns werden durch die Verwendung von $A l G a A s$ oder In GaP Mantelschichten und verschiedenen Dotierprofilen präzise den unterschiedlichen Anforderungen angepasst, entwickelt und realisiert.

Sogenannte vertical external-cavity surface-emitting lasers (VECSEL) ermöglichen Laserbetrieb im Dauerstrichmodus (CW) mit extrem hoher Leistung, perfekt zirkularsymmetrischer Strahlqualität und direkten Zugang zur Laserkavität. Das Konzept ist ideal für eine Vielzahl von Anwendungen, allerdings weisen konventionelle, quantenfilmbasierte VECSEL im Betrieb stets eine hohe Temperaturabhängigkeit auf. Hier werden erstmals VECSEL auf Basis von Submonolagenstrukturen und QP mittels MOVPE realisiert. Mit diesen optisch gepumpten VECSEL wird eine sehr große spektrale Bandbreite von $950 \mathrm{~nm}$ bis $1210 \mathrm{~nm}$ abgedeckt, wobei ein extrem temperaturstabiler Betrieb, aufgrund der Nutzung von QP, und Ausgangsleistungen bis zu 1,4 W CW erreicht werden.

Um die physikalische Limitierung von direkt modulierten, so genannten vertical-cavity surface-emitting lasers zu überwinden, wird ein neuartiges Design mit einem monolithisch 
integrierten elektro-optischen Modulator realisiert. Das Konzept zielt auf die Reflektivitätsmodulation des Auskoppelspiegels ab, um Limitierungen durch Ladungsträgertransport zu umgehen und höchste Modulationsgeschwindigkeiten zu erreichen. Schon erste Prototypen zeigen Leistungen geeignet für $6 \mathrm{~Gb} / \mathrm{s}$ schnelle Datenübertragungen. Eine weiterführende Analyse der Modulationsmechanismen offenbart ein außerordentlich hohes, intrinsisches Bandbreitenpotential von bis zu $56 \mathrm{GHz}$. 


\section{Abstract}

Semiconductor lasers represent the backbone for an increasing variety of applications ranging from information storage and communication, to material treatment. Novel concepts are pushing the limits and are enabling new application areas. Many of these areas demand low-cost laser devices with high-brilliance and high-power light output or high-speed performance.

This thesis demonstrates how key performance characteristics of semiconductor lasers can be tailored using nanostructure design and epitaxial growth. All aspects of laser fabrication are discussed, from design to growth of nanostructures using metal-organic vapor-phase epitaxy (MOVPE), to fabrication and characterization of complete devices. By employing industrial tools, all developed processes are compatible with mass production.

Pulsed high power laser operation up to $8 \mathrm{~W}$ and a ultra-low lasing threshold of $66 \mathrm{~A} / \mathrm{cm}^{2}$ is achieved with electrically pumped quantum dots (QD)-based edge emitters at $1.25 \mu \mathrm{m}$ due to an improved understanding of the QD growth process. This novel process enables $1.3 \mu \mathrm{m}$ edge emitters for telecom applications in the established InGaAs/GaAs system. At the heart of these achievements is the careful investigation and optimization of nearly all layers of the laser device structure. Designs are altered and precisely tuned by employing $A l G a A s$ or $I n G a P$ claddings and varied doping schemes in order to develop new waveguides to meet different requirements.

High-power vertical external-cavity surface-emitting lasers (VECSELs) promise continuous-wave $(\mathrm{CW})$ lasing with perfect circular beam quality, plus direct access to the cavity. While the concept is ideal for a multitude of applications, conventional quantumwell based systems exhibit problematic temperature sensitivity during operation. Here, for the first time, VECSELs with sub-monolayer structures and QDs as active layers are realized by MOVPE. These optically pumped devices cover a wide spectral range from $950 \mathrm{~nm}$ to $1210 \mathrm{~nm}$, and achieve excellent temperature-stable CW lasing due to the use of QDs and CW output powers of up to $1.4 \mathrm{~W}$.

In order to overcome the physical limitations of directly modulated vertical-cavity surface-emitting lasers, a novel concept with a monolithically integrated electro-optical modulator is realized. The concept investigates the possibility of modulating the reflectivity of the top mirror to achieve highest modulation speeds, which are not limited by carrier transport. First prototypes prove to be suitable for up to $6 \mathrm{~Gb} / \mathrm{s}$ data transmission. Subsequent in-depth analysis of the modulation mechanisms reveals an outstanding intrinsic bandwidth potential of up to $56 \mathrm{Ghz}$. 


\section{Publications}

Research, which has already been published:

- A. Strittmatter, T. D. Germann, T. Kettler, K. Posilovic, U. W. Pohl, und D. Bimberg, Alternative precursor metal-organic chemical vapor deposition of InGaAs/GaAs quantum dot laser diodes with ultralow threshold at $1.25 \mu \mathrm{m}$, Applied Physics Letters 88, 262104 (2006)

Selected for the Virtual Journal of Nanoscale Science \& Technology, 14, 2 (2006)

- R. Seguin, A. Schliwa, T. D. Germann, S. Rodt, K. Pötschke, A. Strittmatter, M. Winkelnkemper, T. Hammerschmidt, P. Kratzer, U. W. Pohl und D. Bimberg, Control of fine-structure splitting and excitonic binding energies in selected individual InAs/GaAs quantum dots, Applied Physics Letters 89, 263109 (2006)

Selected for the Virtual Journal of Nanoscale Science \& Technology, 15, 2 (2007)

- T. D. Germann, A. Strittmatter, T. Kettler, K. Posilovic, U. W. Pohl, und D. Bimberg, MOCVD of InGaAs/GaAs quantum dots for lasers emitting close to 1.3 $\mu \mathrm{m}$, Journal of Crystal Growth 298, 591 (2007), (Proc. of the $13^{\text {th }}$ International Conference on Metal Organic Vapor Phase Epitaxy (ICMOVPEXIII), Miyazaki, Japan 2006)

- R. Seguin, A. Schliwa, T. D. Germann, S. Rodt, K. Pötschke, U. W. Pohl, D. Bimberg, Ex-situ control of fine-structure splitting and excitonic binding energies in single InAs/GaAs quantum dots, Physics of Semiconductors, AIP Conf. Proc. 893, 919-920 (2007)

- T. D. Germann, A. Strittmatter, J. Pohl, U. W. Pohl, D. Bimberg, J. Rautiainen, M. Guina, and O. G. Okhotnikov, High-power semiconductor disk laser based on InAs/GaAs submonolayer quantum dots, Applied Physics Letters 92, 101123 (2008)

Selected for the Virtual Journal of Nanoscale Science \& Technology, 17, 12 (2008) 
- A. Strittmatter, T. D. Germann, J. Pohl, U. W. Pohl, D. Bimberg, J. Rautiainen, A. Guina, and O. G. Okhotnikov, $1040 \mathrm{~nm}$ vertical external cavity surface emitting laser based on InGaAs quantum dots grown in Stranski-Krastanow regime, Electronics Letters 44, 290-291 (2008)

Selected for the Virtual Journal of Nanoscale Science \& Technology, 17, 8 (2008)

- T. D. Germann, A. Strittmatter, J. Pohl, U. W. Pohl, D. Bimberg, J. Rautiainen, M. Guina, and O. G. Okhotnikov, Temperature-stable operation of a quantum dot semiconductor disk laser, Applied Physics Letters 93, 051104 (2008)

Selected for the Virtual Journal of Nanoscale Science \& Technology, 18, 7 (2008)

- A. Strittmatter, T. D. Germann, T. Kettler, K. Posilovic, J. Pohl, U. W. Pohl, and D. Bimberg, Suppression of the wavelength blue shift during overgrowth of InGaAs-based quantum dots, Journal of Crystal Growth 310, 5066-5068 (2008), (Proc. of the $14^{\text {th }}$ International Conference on Metal Organic Vapor Phase Epitaxy (ICMOVPE-XIV), Metz, France 2008)

- T. D. Germann, A. Strittmatter, U. W. Pohl, D. Bimberg, J. Rautiainen, M. Guina, and O. G. Okhotnikov, Quantum-dot semiconductor disk lasers, Journal of Crystal Growth 310, 5182-5186 (2008), (Proc. of the $14^{\text {th }}$ International Conference on Metal Organic Vapor Phase Epitaxy (ICMOVPE-XIV), Metz, France 2008)

- T. D. Germann, A. Strittmatter, A. Mutig, A. M. Nadtochiy, J. A. Lott, S. A. Blokhin, L. Ya. Karachinsky, V. A. Shchukin, N. N. Ledentsov, U. W. Pohl, and D. Bimberg, Monolithic electro-optically modulated vertical cavity surface emitting laser with 10 Gbps open-eye operation, Physica Status Solidi (C), 7, 2552-2554 (2010), (Proc. of the International Symposium on Compound Semiconductors (ISCS), Santa Barbara, USA 2009)

- C.-Y. Lu, S.-W. Chang, S. L. Chuang, T. D. Germann, and D. Bimberg, Metalcavity surface-emitting microlaser at room temperature, Applied Physics Letters 96, 251101 (2010)

- C.-Y. Lu, S.-W. Chang, S. L. Chuang, T. D. Germann, U. W. Pohl, and D. Bimberg, CW substrate-free metal-cavity surface microemitters at $300 \mathrm{~K}$, Semiconductor Science and Technology 26, 014012 (2010) 
- S.-W. Chang, C.-Y. Lu, S. L. Chuang, T. D. Germann, U. W. Pohl, and D. Bimberg, Theory of Metal-cavity Surface-emitting Microlasers and Comparison with Experiment, IEEE Journal of Selected Topics in Quantum Electronics 99, 1-12 (2011)

- A. Lenz, H. Eisele, J. Becker, L. Ivanova, E. Lenz, F. Luckert, K. Pötschke, A. Strittmatter, J.-H. Schulze, T. D. Germann, U. W. Pohl, D. Bimberg, and M. Dähne, Atomic structure and optical properties of submonolayer InAs depositions in GaAs, Journal of Vacuum Science and Technology B 29, 04D104 (2011)

- S. L. Chuang, C.-Y. Lu, S.-W. Chang, T. D. Germann, U. W. Pohl, and D. Bimberg, Low Thermal Impedance of Substrate-Free Metal Cavity SurfaceEmitting Microlasers, Photonics Technology Letters 23, 1031 (2011) 


\section{Conferences}

Research, which has already been presented at conferences:

- T. D. Germann, A. Strittmatter, T. Kettler, K. Posilovic, K. Schatke, K. Pötschke, D. Feise, U. W. Pohl, and D. Bimberg, Entwicklung der Oberflächenmorphologie von InGaAs/GaAs - Quantenpunktstrukturen, Abstracts of the "Epitaxie von III-V-Halbleitern" workshop of the Deutsche Gesellschaft für Kristallwachstum und Kristallzüchtung (DGKK), Duisburg, Germany (2005)

- T. D. Germann, A. Strittmatter, T. Kettler, K. Posilovic, and D. Bimberg, Stacking of InGaAs/GaAs-based quantum dots for long-wavelength laser diodes, semiconductor physics section at spring-meeting of the German Physical Society (DPG), Dresden, Germany (2006)

- T. D. Germann, A. Strittmatter, T. Kettler, K. Posilovic, U. W. Pohl, and D. Bimberg, Transferring achievements of quantum dot test structures to laser devices, semiconductor physics section at spring-meeting of the German Physical Society (DPG), Regensburg, Germany (2007)

- T. D. Germann, A. Strittmatter, U. W. Pohl, and D. Bimberg, Nanoscale Stacking of InGaAs/GaAs Quantum Dots, Symposium on Vacuum based Science and Technology by Deutsche Vakuumgesellschaft (DVG), Greifswald, Germany (2007)

- T. D. Germann, A. Strittmatter, J. Pohl, U. W. Pohl, and D. Bimberg, Design und Wachstum von Quantenpunkten für Hochleistungs - VECSEL, Abstracts of the "Epitaxie von III-V-Halbleitern" workshop of the Deutsche Gesellschaft für Kristallwachstum und Kristallzüchtung (DGKK), Marburg, Germany (2007)

- T. D. Germann, A. Strittmatter, J. Pohl, U. W. Pohl, D. Bimberg, J. Rautiainen, M. Guina, and O.G. Okhotnikov Quantum-Dot Semiconductor Disk-Lasers, Ext. Abstracts of the International Nano-Optoelectronics Workshop (iNOW), Tokyo, Japan (2008) 
- T. D. Germann, A. Strittmatter, A. Mutig, A. M. Nadtochiy, J. A. Lott, S. A. Blokhin, L. Ya. Karachinsky, V. A. Shchukin, N. N. Ledentsov, U. W. Pohl, and D. Bimberg, Development of an electro-optically modulated vertical cavity surface emitting laser, Ext. Abstracts of the International Nano-Optoelectronics Workshop (iNOW), Stockholm and Berlin, Sweden and Germany (2009)

- T. D. Germann, J.-H. Schulze, A. Mutig, A. M. Nadtochy, S. A. Blokhin, J. A. Lott, V. A. Shchukin, N. N. Ledentsov, U. W. Pohl, and D. Bimberg, Realization of monolithic electro-optically modulated vertical cavity surface emitting lasers, $15^{\text {th }}$ International Conference on Metal Organic Vapor Phase Epitaxy (ICMOVPE-XV), Lake Tahoe, USA (2010)

- T. D. Germann, U. W. Pohl, D. Bimberg, C.-Y. Lu, S.-W. Chang, and S. L. Chuang, MOVPE of a metal-cavity surface-emitting laser operating $\mathrm{cw}$ at room temperature, $15^{\text {th }}$ International Conference on Metal Organic Vapor Phase Epitaxy (ICMOVPE-XV), late news contribution, Lake Tahoe, USA (2010) 


\section{Patents}

Invention disclosures reported to the Technische Universität Berlin:

- A. Strittmatter, T. D. Germann, J.-H. Schulze, A. Schliwa, U. W. Pohl, V. A. Gaisler,

Verfahren zur Positionierung von Nanostrukturen

submitted, 12/2010

accepted by IPAL $\mathrm{GmbH}$ for patent process

Application was filed on 03/30/2011 with title LAYER ASSEMBLY

as United States Patent Application Number 13/076,166

- A. Strittmatter, J.-H. Schulze, T. D. Germann,

Halbleiter-Laserdioden und optische Verstärker mit lateral strukturierter

Gewinnzone

submitted, 02/2011

accepted by IPAL $\mathrm{GmbH}$ for patent process

Application was filed on 06/28/2011 with title Elektrooptisches Bauelement as German Patent Application Number 102011078176.5 


\section{Awards}

Awards received for accomplished research:

- Best poster award, $2^{\text {nd }}$ place at the International Nano-Optoelectronic Workshop (iNOW) 2008, Japan

T. D. Germann, A. Strittmatter, J. Pohl, U. W. Pohl, D. Bimberg, J. Rautiainen, M. Guina, and O. G. Okhotnikov,

Quantum-Dot Semiconductor Disk-Lasers

- Best poster award, $1^{\text {st }}$ place at the International Nano-Optoelectronic Workshop (iNOW) 2010, China

C.-Y. Lu, S.-W. Chang, S. L. Chuang, T. D. Germann, U. W. Pohl, and D. Bimberg,

Substrate-Free Metal Cavity Surface Emitting Microlaser at Room Temperature

- Dimitris N. Chorafas Foundation Award 2010, Germany

for exceptional doctoral research on the

Design and realization of novel semiconductor laser concepts 



\section{Contents}

1 Introduction 1

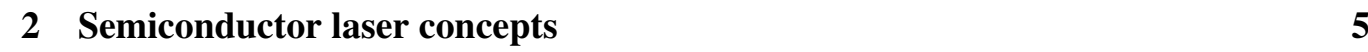

2.1 Evolution of semiconductor lasers $\ldots \ldots \ldots \ldots \ldots$

2.2 Gain concepts . . . . . . . . . . . . . . . . . . . . 6 6 6

$2.2 .1 \quad$ Quantum wells $\ldots \ldots \ldots \ldots \ldots$. . . . . . . . . . . . 6

2.2 .2 Quantum dots . . . . . . . . . . . . . . . 7

2.2 .3 Sub-monolayer structures $\ldots \ldots \ldots \ldots$

2.3 Semiconductor laser basics . . . . . . . . . . . . . . . . . . . . . 10

2.3 .1 Edge-emitting lasers $\ldots \ldots \ldots \ldots \ldots \ldots$

2.3 .2 Surface-emitting lasers $\ldots \ldots \ldots \ldots \ldots$. . . . . . . . . 12

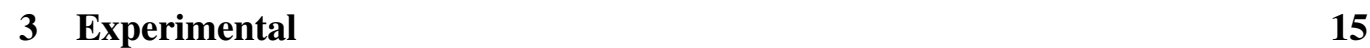

$3.1 \quad$ Metal Organic Vapor Phase Epitaxy $\ldots \ldots \ldots \ldots$. . . . . . . 15

$3.1 .1 \quad$ QD growth process $\ldots \ldots \ldots \ldots \ldots \ldots$

3.1 .2 Wafer temperature calibration . . . . . . . . . . . . 18

3.2 Optical characterization . . . . . . . . . . . . . . . . . . . . . . . . . 19

3.3 Sample structure design $\ldots \ldots \ldots \ldots \ldots$. . . . . . . . . . . 21

4 MOVPE processes 25

4.1 Quantum dot growth . . . . . . . . . . . . . . . . . 25

$4.1 .1 \quad$ QD optimization close to $1300 \mathrm{~nm}$. . . . . . . . . . . . . 25

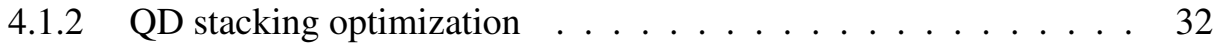

4.2 Doping and material quality $\ldots \ldots \ldots \ldots$. . . . . . . . . 40

$4.2 .1 \mathrm{GaAs} \ldots \ldots \ldots \ldots \ldots \ldots \ldots$

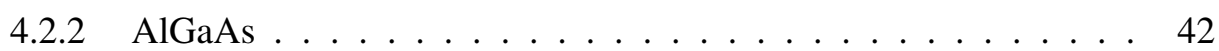

$4.2 .3 \quad \mathrm{InGaP} \ldots \ldots \ldots \ldots \ldots \ldots \ldots$

5 Edge-emitting quantum dot lasers 49

$5.1 \quad$ Broad waveguide lasers . . . . . . . . . . . . . . . . . . . . . . 49

$5.1 .1 \quad$ Aluminum-free laser design $\ldots \ldots \ldots$. . . . . . . . 57

$5.2 \quad$ Laser diodes at $1.3 \mu \mathrm{m} \ldots \ldots \ldots$. . . . . . . . . . . 6 60

$5.2 .1 \quad$ QD blue shift suppression . . . . . . . . . . . . . . 63

5.2 .2 Substrate size effects $\ldots \ldots \ldots \ldots \ldots$. . . . . . . 67 
$5.2 .3 \quad 1.3 \mu \mathrm{m}$ laser design $\ldots \ldots \ldots \ldots \ldots$

5.3 Summary and Outlook $\ldots \ldots \ldots \ldots$. . . . . . . . . . 73

$6 \quad$ High-power vertical external-cavity surface-emitting lasers 75

$6.1 \quad$ Device mounting and characterization . . . . . . . . . . . . . . 77

$6.1 .1 \quad$ ORC VECSEL setup . . . . . . . . . . . . . . . . . . . 77

$6.1 .2 \quad$ TU-Berlin VECSEL setup $\ldots \ldots \ldots \ldots$. . . . . . . . . 77

6.2 Initial nonlinear gain structure design $\ldots \ldots \ldots \ldots$. . . . . . 80

$6.2 .1 \quad$ Active media . . . . . . . . . . . . . . . . . 80

$6.2 .2950 \mathrm{~nm}$ SML-VECSEL $\ldots \ldots \ldots \ldots \ldots$. . . . . . . . . 81

$6.2 .3 \quad 1040 \mathrm{~nm}$ SML-VECSEL $\ldots \ldots \ldots \ldots$. . . . . . . . . . 83

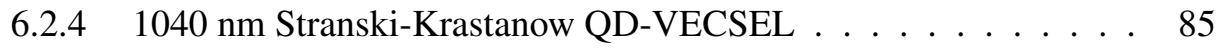

6.2 .5 Temperature dependence and lifetime $\ldots \ldots \ldots \ldots \ldots$

6.3 Long wavelength gain structure design $\ldots \ldots \ldots$. . . . . . . . . . . 94

$6.3 .1 \quad 1210 \mathrm{~nm}$ Stranski-Krastanow QD-VECSEL . . . . . . . . . . . . 94

6.3 .2 Temperature stability . . . . . . . . . . . . . . . . . . . 97

6.4 Conclusions . . . . . . . . . . . . . . . . . . . . . . . 101

$\begin{array}{lll}7 & \text { Electro-optically modulated vertical-cavity surface-emitting lasers } & 103\end{array}$

$7.1 \quad$ Electro-optical modulator . . . . . . . . . . . . . . . . . . . . . . . . . . 104

7.1 .1 Quantum confined Stark effect . . . . . . . . . . . . . . . . 104

7.1 .2 Modulator design . . . . . . . . . . . . . . . . . . 105

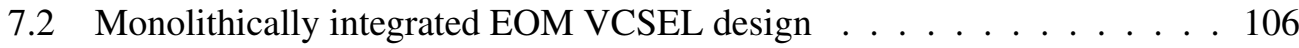

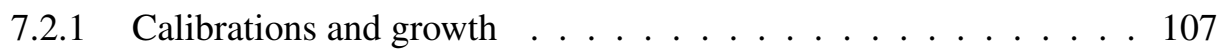

7.2 .2 Processing and characterization $\ldots \ldots \ldots \ldots \ldots$

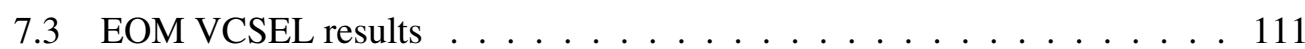

$7.3 .1 \quad$ High speed operation $\ldots \ldots \ldots \ldots \ldots$

7.3 .2 Static modulation . . . . . . . . . . . . . . . . . . . . . 113

7.3 .3 Limitation analysis . . . . . . . . . . . . . . . . . . . . . 114

7.3 .4 Resonant electro-optic modulation . . . . . . . . . . . . . . 116

7.4 Conclusions . . . . . . . . . . . . . . . . . . . . 123

\begin{tabular}{lll}
\hline & Summary and Outlook & 127
\end{tabular}

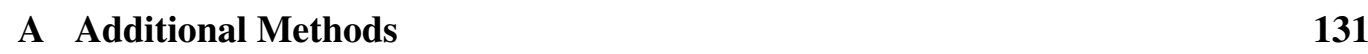

A.1 MOVPE growth . . . . . . . . . . . . . . . . . . . . . . . 131

A.1.1 In-situ control . . . . . . . . . . . . . . . . . . . . 131

A.1.2 Process design tools . . . . . . . . . . . . . . . . 132

A.2 Sample characterization . . . . . . . . . . . . . . . . . 133 
A.2.1 Microscopic characterization . . . . . . . . . . . . 133

A.2.2 Volume-crystal characterization . . . . . . . . . . . . . . 134

A.3 Sample structure designs . . . . . . . . . . . . . . . . . . . . 135

A.4 Transfer matrix simulation . . . . . . . . . . . . . . . . . . . . 136

A.5 Processing and device characterization . . . . . . . . . . . . . 136

A.5.1 Edge-emitter fast-process . . . . . . . . . . . . . . . . 137

A.5.2 Edge emitter characterization . . . . . . . . . . . . 137

\begin{tabular}{ll}
\hline References & 139
\end{tabular}

\begin{tabular}{ll}
\hline Acknowledgments & 163
\end{tabular} 



\section{Chapter 1}

\section{Introduction}

Lasers are devices that emit radiation through an amplification process by stimulated emission of photons. The term laser as an acronym for Light Amplification by Stimulated Emission of Radiation was first introduced in the 1950s by G. Gould [Gou59], however A. Schalow and C. Townes had theoretically developed the idea of a laser as an infrared or optical maser prior to the term being coined [Sch58]. The first successful experimental demonstration of this principle was the ruby laser in 1960 by T. H. Maiman [Mai60].

Currently a wide variety of different realizations of the laser principle exist. The most widespread type is the semiconductor laser diode. Commercial use of these semiconductor lasers at room temperature (RT) in everyday life is made possible by the breakthrough invention of the double hetero structure (DHS) concept, which saw the Nobel prize awarded to H. Kroemer and Z. Alferov in 2000 for the theoretical proposal and first experimental realization [Kro63, Alf69, Alf71]. This fundamental concept is further enhanced by the introduction of separate confinement heterostructure $(\mathrm{SCH})$ laser designs, which have significantly improved device performance by separating optical and electrical confinement within the structure. Cost efficient manufacturing of such SCH-laser designs based on semiconductor nanostructures only became possible since the 1980s through industrial scale epitaxial crystal growth. Laser diodes based on these concepts are essential for present-day global infrastructure such as optical data interconnects, high density optical storage media such as CD, DVD, and Blu-Ray discs, novel medical applications such as tumor treatment, high precision measurement systems and material treatment.

In order to satisfy many kinds of applications with diverging requirements several device concepts evolved. The most common laser designs are based on quantum wells (QWs) as active light emitting semiconductor nanostructures. Besides QWs, which are essentially a very thin DHS with an enclosed layer thickness of only a few nanometers, other nanostructure concepts such as quantum dots (QDs) have been employed as active media in lasers since the 1990s [Kir94]. QDs are predicted to enable temperature stability, highest gain and differential gain, as well as lowest threshold current densities [Ara82, Asa86]. Due to the inhomogeneous size distribution of QDs grown in the epitaxially predominant Stranski-Krastanow (SK) growth mode [Str38], the gain of a single QD layer is distributed over a wide spectral range. Thus, the narrow peak gain of an individual QW layer is, in almost all cases, superior to the peak gain of a single QD layer. However, for laser designs 
requiring a perfect match of spectral gain with a narrow cavity resonance the broad QD gain proves to be a huge advantage. Additionally, QDs from a single material system such as InGaAs/GaAs are tunable over a wide spectral range while sustaining good optical quality and gain for laser applications. In order to combine high peak gain and the intrinsic advantages of the three-dimensional QD confinement within one structure, so called sub-monolayer (SML) structures were proposed, and were recently used for first laser applications [Hop07, Ger08b].

The work described here focuses on the development of advanced epitaxial QD growth processes and the realization of three completely different $G a A s$ based laser design concepts by metal-organic vapor-phase epitaxy (MOVPE). As a result of the strongly diverging MOVPE growth process objectives a multitude of challenges are faced, which are outlined below:

Growth mechanisms of In GaAs SK-QDs are investigated in detail with the goal of long wavelength lasing around $1.3 \mu \mathrm{m}$. The challenge was to develop a SK-QD growth process employing MOVPE, which provides stacked QDs with sufficient gain for laser applications at that wavelength. Two key issues needed to be overcome to push high quality $\operatorname{In} G a A s$ QDs towards $1.3 \mu \mathrm{m}$ : maintenance of a high areal QD density of at least $10^{10} \mathrm{~cm}^{-2}$, an increase of total gain via a stacking process for closely grouped QD layers. This minimum QD density value is based on molecular beam epitaxy (MBE) data, as prior to this work only MBE grown lasers at this wavelength had been reported [Huf98, Muk99, Lot00].

The only reports of MOVPE grown QD lasers at $1.3 \mu \mathrm{m}$ emerged recently using a sophisticated antimony mediated growth process and 5 to 10 QD layers [Gui07, Gui08]. For the realization of MOVPE grown QD lasers at $1.3 \mu \mathrm{m}$ the main obstacle is the unintended blue shift of the QD emission upon overgrowth and annealing. The goal of this research was the development of a MOVPE process for long wavelength edge-emitting InGaAs-QD lasers with a complete suppression of any blue shift upon device integration and without the need for additional mediating materials.

The second device project was aimed at the realization of high-power vertical externalcavity surface-emitting lasers (VECSELs) based on novel gain material concepts for emission wavelengths at $940 \mathrm{~nm}, 1040 \mathrm{~nm}$, and $1220 \mathrm{~nm}$. Existing VECSELs based on QW gain material suffer from poor temperature stability due to the complex interaction of gain chip cavity, external cavity, and narrow spectral gain [Kuz99, Tro04]. Here, alternative QD and SML based VECSEL are developed to overcome this inherent limitation. The main challenge for MOVPE growth is the development of extremely thick active cavities including $>10$ active layers of either SK-QDs or SML structures to compensate for the lower peak gain compared to QWs.

In order to overcome the physical modulation-speed limitation of directly-modulated vertical-cavity surface-emitting lasers (VCSELs), the novel concept of a monolithically- 
integrated electro-optical modulator (EOM) was investigated and fabricated. This EOM VCSEL requires the ultra-precise alignment of active-cavity and modulator-cavity resonances to $<1 \mathrm{~nm}$ within an extensive device design that contains close to 400 individual layers and has a total thickness of several microns. Additionally, to allow interpretation of results from such a multi-cavity EOM VCSEL concept, extensive characterization is required to assess the intrinsic device properties.

\section{Chapters of this work are organized as follows:}

Chapter 2 An introduction into the fundamental concepts of semiconductor laser designs and the physical properties of the employed nanostructures is given.

Chapter 3 Employed experimental methods are described with special focus on the requirements of epitaxial process optimization and the basic QD growth process.

Chapter 4 Long wavelength In GaAs QD growth process optimization for applications at $1.3 \mu \mathrm{m}$ is investigated. Notably, the role of the V/III ratio was found to be crucial for the long-term stability of QD properties during subsequent QD overgrowth and annealing. Results of MOVPE grown material properties used for device fabrication and limits of possible doping levels are outlined.

Chapter 5 Experimental results from the developed edge-emitting laser-diodes using the highly optimized new QD growth process are presented. Complete blue-shift suppression is achieved for long wavelength QDs. Stacks of QD layers emitting at $1.3 \mu \mathrm{m}$ are grown which show complete wavelength stability upon overgrowth or annealing at $615^{\circ} \mathrm{C}$ and are used within a laser device.

Chapter 6 For the first time, MOVPE grown VECSELs using SMLs and QDs as active media are realized. Results of VECSEL for $950 \mathrm{~nm}, 1040 \mathrm{~nm}$, and $1210 \mathrm{~nm}$ emission wavelengths are presented. QD VECSEL especially benefit from the very broad gain spectrum, enabling almost perfect temperature stability during operation.

Chapter 7 The realization of an EOM VCSEL for high-speed applications is shown. An in-depth device characterization is presented along with the growth and optimization challenges encountered for this $\approx 400$ layer dual cavity design. Modeling and measurements reveal existing prototype limitations and predict an intrinsic bandwidth potential of $56 \mathrm{GHz}$ for future devices. 



\section{Chapter 2}

\section{Semiconductor laser concepts}

Fundamentals of $G a A s$-based laser designs and the investigated $(\operatorname{In})(G a) A s$ gain media concepts are discussed within this chapter. $(A l) G a A s$ is the material system which is primarily employed for the infrared spectral range. Due to its versatility and ability to form dielectric mirrors for vertically emitting devices, $(A l) G a A s$ forms the basis for a wide range of applications in the near infrared spectrum, and is well-established for industrial mass production.

\subsection{Evolution of semiconductor lasers}

Since its inception, some of the main goals behind semiconductor laser development have been the creation of new designs to achieve reduction of the lasing threshold, increase in modulation speed, and higher output power. Well known examples in everyday life include the AlGaAs laser diodes operated in continuous-wave mode (CW) at $780 \mathrm{~nm}$ employed for compact discs, and at $848 \mathrm{~nm}$ for laser computer mice. Optical interconnects driving the Internet rely completely on infrared semiconductor laser technology, and steady demand exists for higher modulation speeds and more cost-efficient devices. All of these examples are based on QW active media. In parallel to this quasi standard in today's industry, more sophisticated nanostructures such as QDs have been introduced as a step to improving laser performance and to unlocking new application areas. While the evolution of QD lasers started In the 1990s, the first QD devices are just now entering the market.

Lithographic techniques and chemical wet etching with subsequent overgrowth were used to fabricate the first QD lasers. These structures showed pulsed lasing at $77 \mathrm{~K}$ with extremely high $j_{t h}$ of $7.6 \mathrm{kA} / \mathrm{cm}^{2}$ [Hir94]. A significant advance in terms of reducing $j_{t h}$ was the use of self organized QD growth in the Stranski-Krastanow growth mode (SK) [Str38], which allowed for an essential reduction in the defect density within the QD layer. A 942 nm SK-QD laser using MBE growth was first developed by Kirstaedter et al., and demonstrated a significantly reduced $j_{t h}$ of $120 \mathrm{~A} / \mathrm{cm}^{2}$ at $77 \mathrm{~K}$ and $950 \mathrm{~A} / \mathrm{cm}^{2}$ at RT [Led94, Kir94]. This breakthrough started a series of reports on improved MBE-grown SK-QD lasers with $j_{t h}$ down to $19 \mathrm{~A} / \mathrm{cm}^{2}$, realized with aluminum-oxide confinement layers and emission wavelength up to $1.3 \mu \mathrm{m}$ [Les99, Par00]. While the extremely low threshold characteristics and long wavelength emission around $1.3 \mu \mathrm{m}$ of these QD lasers 
were predominantly achieved by MBE-grown devices (an overview can be found in [Kai06]), the first successful MOVPE-based fabrication of SK-QD lasers emerged in 1997 [Hei97b]. Steady development of QD devices in the following years enabled success in significantly improved MOVPE-based SK-QD laser processes to close the gap to MBE devices [Kai04, Str06].

\subsection{Gain concepts}

Besides $(A l) G a A s$ itself, the dominant active material for $G a A s$-based devices are In $G a A s$ quantization layers. Electronic states show quantization effects if at least one dimension of an enclosed nanostructure is reduced to less than the de Broglie wavelength of the confined charge carrier (electron: $\lambda=h / p \leq 30 \mathrm{~nm}$ ). Such nanostructures of reduced dimensionality can be epitaxially grown as thin insertions within a matrix material, e.g. indium containing material can be grown as pseudomorphically strained layers. Due to the reduced band gap energy of InGaAs as compared to GaAs, electrical confinement is easily achieved. In order to fabricate customized active layers different approaches can be taken. The active layer concepts employed within devices described in this work are briefly outlined below.

\subsubsection{Quantum wells}

Physically a QW is realized if a sufficiently thin layer confines charge carriers in the dimension perpendicular to its surface, while in-plane movements within the layer are possible. In contrast to bulk material, the density of states changes from a square root to a step-like function, thus the density of states becomes constant for the energy intervals between energetic eigenstates. The resulting electronic properties of such a QW can be tuned by its thickness, and are not solely dependent on material properties, such as is the case for bulk layers.

The main obstacles for highest QW quality growth are smooth interfaces [Bim92] and dislocation free layers in heavily strained systems. Nevertheless, growth of $\mathrm{QW}$ in the In GaAs/(Al)GaAs and GaAs/AlGaAs is well-established and understood. QW energy levels, optical gain, and other properties can be analytically calculated [Mak96]. For positive values ( $g_{\text {material }} \geq 0$ ), the material gain of a $\mathrm{QW}$ can be closely approximated by a logarithmic function using an empirical gain coefficient $g_{0}$, pump current-density $j$, transparency current-density $j_{t r}$, and by neglecting saturation and occupation of excited states [Col95, Bac07].

$$
g_{\text {material }}=g_{0} \cdot \ln \frac{j}{j_{t r}}
$$


Optical gain of a single QW is often sufficient to reach lasing threshold, whereas for high power applications or vertical designs multi $Q W$ stacks (MQW) can be used as active media. From the point of view of epitaxial-growth, the number of QWs and the thickness of the spacers within a MQW are limited only by the total incorporated strain. This can be partially addressed by the introduction of strain-compensating layers. Thus, the spectral tuning range of QWs is also mainly limited by strain, as maximum thickness and lattice mismatch of a grown layer are given by the accumulated elastic energy required for dislocation formation, and thus relaxation of the grown QW layer.

\subsubsection{Quantum dots}

QDs confine charge carriers in all three dimensions within the length of the de Broglie wavelength, and the density of states in QDs is described by a $\delta$-function instead of the step function of QWs. Consequently, they are often called zero-dimensional structures. Thus, discrete energy levels comparable to that of a single atom exist for a QD. Using the density-matrix theory Asada at al. calculated the QD gain and predicted up to ten-fold higher values for QDs as compared to equivalently thick QWs [Asa86]. However, this advantage is limited by the fact that the occupied volume of QDs is significantly smaller than that of a QW. For a very high QD density of $1 \times 10^{11} \mathrm{~cm}^{-2}$ and lateral QD base area of $100 \mathrm{~nm}^{2}$ the volume is one tenth of an equally high QW. As a consequence, the overlap with the confined optical field, known as the confinement factor $\Gamma$, shrinks. Thus, the modal gain $g$ is reduced equivalently as it is given by the product of the confinement factor and the material gain.

$$
g=g_{\text {material }} \cdot \Gamma
$$

The gain is also affected by the inhomogeneous broadening of the QD energy spectra to several tens of meV, caused by the size distribution of the QD ensemble. Consequently, only a part of the available QDs can contribute to the cavity mode of a laser. Additionally, finite confinement potentials render the escape of carriers into the barrier possible. An analytical description of the gain spectrum $g(\hbar \omega)$ is discussed by Bimberg et al. based on the work of Yan et al. and Chuang [Bim98, Yan90, Chu09, Asr01]. Although it is beyond the scope of the current work, profound overviews on QD properties, applications and the Stranski-Krastanow (SK) growth mode [Str38] can be found in these books: [Bim98, Gru02, Bim08]. A theoretical model on the strain driven QD island formation has been published by Shchukin et al. [Shc95, Shc98a].

Currently, the basic physical mechanisms of the QD 2-D to 3-D transition after reaching a critical layer thickness of $\approx 1.7$ monolayers (MLs) of InAs are understood [Leo94, Hei98a]. However, epitaxial QD growth processes are not yet able to completely control for all QD properties, such as emission wavelength and areal density independently of each other. Thus it is of fundamental interest to advance knowledge on the influence of QD 


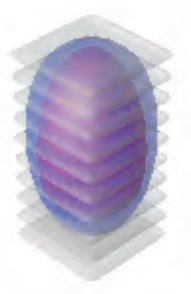

(a)

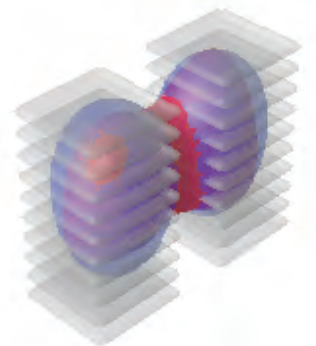

(b)

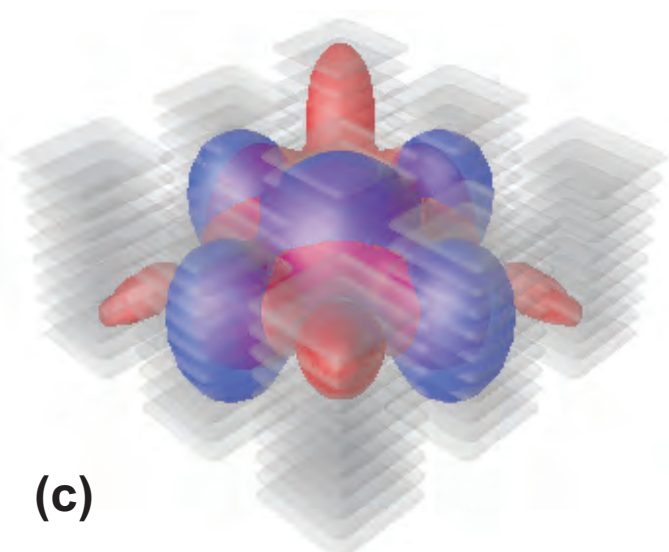

Figure 2.1: Schematic of the lateral coupling of InAs/GaAs SML structures based on calculations of electron (blue) and hole (red) wave-functions by Dr. Andrei Schliwa. In the vertical plane InAs islands are separated by $3 \mathrm{ML} \mathrm{GaAs}$, laterally a distance of $2 \mathrm{~nm}$ is set. (a) Isolated 10-fold SML structure. (b) A pair of SML structures shows lateral coupling. (c) Wave functions extend laterally across the $3 \times 3$ SML configuration.

growth parameters on final structural and optical QD properties. During the overgrowth process QDs become three-dimensional inclusions within the surrounding semiconductor matrix crystal. But in contrast to QW epitaxy, which shows always a flat growth front, all QD layer overgrowth processes start with a three dimensionally shaped, and thus rough surface, which needs to be flattened during spacer growth prior to the following QD layer being grown. In consequence, advanced growth processes are necessary to deal with this challenge.

As previously mentioned, the inherent QD size distribution as a result of the selforganized SK process causes QD layers to show a much broader luminescence when compared to QWs. Thus, the peak gain per QD layer is lower than for QWs, and QD stacking is often required. Whereas the broad spectral gain width can be used to provide a unique advantage for QD-based VECSELs, as is shown in chapter6. Another intrinsic advantage of QDs is the very widely accessible spectral range. A single material system such as InGaAs/GaAs enables the fabrication of lasers for the entire wavelength range from $\approx 1000 \mathrm{~nm}$ up to $\approx 1350 \mathrm{~nm}$ by tuning QD growth parameters.

\subsubsection{Sub-monolayer structures}

In contrast to QWs and QDs, research on SML structures as active media is rather young. Comprehensive reviews on SML self-organization, optical properties, and applications have been published by Krestnikov et al. and Ledentsov et al. [Kre01, Led07]. SML structures consist of a superlattice of SML depositions of low band-gap material separated 

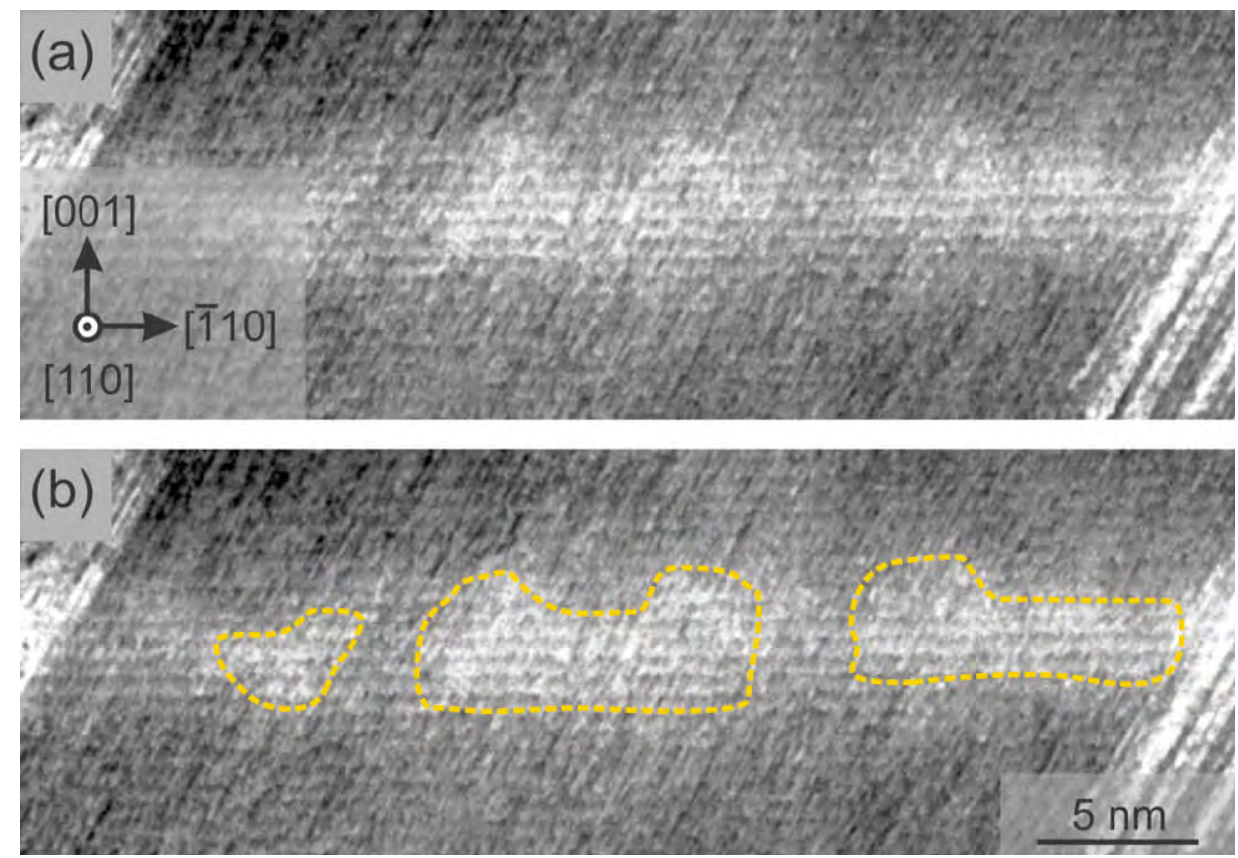

Figure 2.2: (a) and (b) are identical XSTM images, in (b) InAs-rich agglomerations are indicated by yellow dashed lines as a guide to the eye. Close-view filled-state XSTM images of a 10-fold SML stack with $0.5 \mathrm{ML}$ InAs and $1.5 \mathrm{ML}$ GaAs spacers per iteration, taken at 110 cleavage surface using $V_{S}=-2.6 \mathrm{~V}$ and $I_{T}=60 \mathrm{pA}$. This work has been published in [Len11].

by few MLs of thick matrix material spacer layers. All iterated SML depositions consist of less material than is needed to form a complete ML on the surface. Thus, the initial SML deposition is obliged to form islands where the size of these SML islands is defined by the minimization of the total energy of the system. While the surface energy component depends only on the amount of deposited material, the boundary and strain energies depend on the island size [Shc99, Kre01]. For all subsequently iterated SML depositions the term $E_{\text {strain }}^{\text {interaction }}$ is additionally applied for the elastic energy resulting from the interaction of surface islands with buried islands, leading to correlations and anti-correlations within the SML structure [Shc98b]. While a single SML island is too small to confine an electron, the close correlation of these InAs islands in the vertical plane upon stacking ultimately leads to the formation of a three-dimensional structure capable of acting as a QD within the crystal (cf. schematic figure 2.1).

Theoretical models can explain the physics of SML formation and SML wave-functions, however, an understanding of epitaxial growth processes and their parameters in controlling SML optical characteristics is in the early stages. Structural investigations and optical characterization of MBE-grown InGaAs/GaAs SMLs describe the fabricated structures as a mixed state of QDs enclosed within a QW showing distinct QD properties, which are overlapped by the stronger QW luminescence at higher excitation levels [Xu03a, Xu03b]. 
MOVPE-grown SML structures described in this work use nominally the same amount of 0.5 ML InAs per SML deposition cycle as in the cited references, however the optical properties are dominated by $\mathrm{QW}$-like characteristics. A theoretical assessment predicts an intended ultra-high SML island density of $\approx 10^{12} \mathrm{~cm}^{-2}$, and an average distance between these InAs islands of only $2 \mathrm{~nm}$ for this amount of material. This narrow lateral separation allows for lateral electronic coupling between neighboring SML island stacks, leading to optical properties at high excitations which are comparable to very rough QWs [Xu06]. A schematic representation based on calculations by Andrei Schliwa is given in figure depicting the coupling of neighboring SML stacks 2.1. Investigations of our samples by cross sectional scanning tunneling microscopy (XSTM) confirmed the predicted narrow separation between adjacent InAs-rich agglomerations as shown in figure 2.2. Individual SML depositions are not resolved in the XSTM picture of this SML structure due to the very thin spacing of 1.5 ML GaAs, and the indium segregation in the growth direction. Detailed results from these SML investigations by XSTM are published in [Len11]. Similar SML structures are used as active media for the SML VECSELs presented in chapter 6

\subsection{Semiconductor laser basics}

All modern SCH-semiconductor laser designs have to achieve both confining photons, and confining charge carriers according to their respective wavelengths. In order to confine photons, the typical thickness of a cavity extends to a few hundred nanometers on the order of $\approx \lambda / n$ while electrons require nanostructures with dimensions about one order of magnitude smaller according to the de-Broglie wavelength $\lambda=h / p$. Thus, it is crucial to position nanostructures at the maximum of the confined optical field for optimum modal gain (cf. equation 2.2). The lasing threshold is achieved if the modal gain equals the internal losses $\alpha_{i}$ plus the mirror losses $\alpha_{\text {mirror }}$ of the laser. With mirror reflectivity $R_{x}$ and cavity length $L$ this can be written as:

$$
g\left(j_{t h}\right)=\alpha_{i}+\alpha_{\text {mirror }}=\alpha_{i}+\frac{1}{2 L} \ln \left(\frac{1}{R_{1} R_{2}}\right)
$$

Using equations 2.1 and 2.2 the threshold current-density $j_{t h}$ can be written as:

$$
j_{t h}=j_{t r} \cdot \exp \left(\frac{\alpha_{i}}{\Gamma g_{0}}+\frac{1}{\Gamma g_{0}} \cdot \frac{1}{2 L} \ln \left(\frac{1}{R_{1} R_{2}}\right)\right)
$$

The transparency current density is the current density which renders the laser neither optically absorbing, nor optically amplifying; thus the laser is transparent. To experimentally determine gain values and transparency current density $j_{t r}$ of edge emitters, several edge-emitters of different lengths were cleaved to enable the measurement of a set of lasers with different cavity lengths. By interpolating the threshold current density $j_{t h}$ values 
of the measured devices to infinite cavity length, the transparency current density can be directly determined from the following equation 2.4 .

$$
j_{t r}=\frac{j_{t h}(L=\infty)}{\exp \left(\frac{\alpha_{i}}{\Gamma g_{0}}\right)}
$$

Another important laser parameter is the internal quantum efficiency $\eta_{i n t}$, which is defined as the ratio of the internally-emitted photons to electron-hole pairs injected into the p-n junction of the laser diode. In the experiment, only the out-coupled photons, which are equivalent to the mirror losses $\alpha_{\text {mirror }}$, can be measured. The ratio of the out-coupled photons to the injected electron-hole pairs is defined as differential quantum efficiency $\eta_{\text {diff }}:$

$$
\eta_{\text {diff }}=\eta_{\text {int }} \cdot \frac{\alpha_{\text {mirror }}}{\alpha_{\text {mirror }}+\alpha_{i}}
$$

Experimentally $\eta_{\text {diff }}$ is deduced from the linear slope of the optical output power as a function of the pump current above the lasing threshold:

$$
\eta_{\text {diff }}=\frac{\Delta P}{\Delta I} \cdot \frac{e}{h \nu}
$$

Measured $\eta_{\text {diff }}$ values for lasers with different cavity lengths can then subsequently be used to determine $\eta_{i n t}$ and $\alpha_{i}$. Using the equation 2.6 and the definition of the mirror losses from equation $2.31 / \eta_{\text {diff }}$, this can be written as:

$$
\frac{1}{\eta_{\text {diff }}}=\frac{1}{\eta_{\text {int }}}-\left(\frac{1}{\ln \left(R_{1} R_{2}\right)} \cdot \frac{2 \alpha_{i}}{\eta_{\text {int }}}\right) \cdot L
$$

Now $1 / \eta_{d i f f}$ can be drawn as a function of $L$ and linearly fitted, this directly gives for $L=0$ a value for $1 / \eta_{\text {int }}$ and allows use of the slope to calculate $\alpha_{i}$.

Aside from using their fundamental physical device properties, semiconductor lasers can be assigned to two distinct groups:

- Edge-emitting lasers: Light within the cavity propagates parallel to the semiconductor wafer surfaces, and thus also parallel to all epitaxial grown layers. Cleaved wafer facets are used to reflect and/or couple out laser light. Device cavities of several $\mathrm{mm}$ in length can be fabricated due to light propagation along the active zone in order to increase total gain.

- Surface-emitting lasers: The cavity axis is perpendicular to the semiconductor wafer surface and light is coupled out through the epitaxial surface or the bottom of the wafer. As light propagation is also perpendicular to the active layers, the pumped active area and the achievable number of grown active layers limit output powers. 
In addition to this fundamental classification, a multitude of application-specific designs exist to generate appropriate device characteristics. For all theoretical concepts the design inherently defines the maximal laser properties which are attainable. Subsequently, growth and processing expertise need to attain the given intrinsic optimum e.g. by achieving maximum gain, eliminating defects, and providing sufficient cooling. In the following, the basic characteristics, possibilities, and limits for the fabricated lasers described within this work are discussed.

\subsubsection{Edge-emitting lasers}

For all edge-emitting lasers light propagation is in-plane, as epitaxial processes offer only vertical structuring, additional lateral confinement is required. While the vertical confinement is realized by epitaxially grown cladding layers with a lower refractive index, the lateral confinement is set up post-growth by etching stripes into the wafer surface. In this manner, index guiding in the vertical and lateral directions is achieved (gain guiding concepts are also possible). However, as the vertical extension of the waveguide is significantly smaller than the processed lateral extension, the asymmetric beam shape limits coupling capabilities to optical fibers. A current guiding effect is also realized by the etching of the top-cladding layer, which is limited by the fact that charge carriers can still diffuse laterally within the waveguide. By simply using cleaved facets as perfectly plane-parallel mirrors to form the resonator, no epitaxial or subsequently processed mirrors are required for laser operation. Many variants of this basic edge-emitter concept exist to enable specific features, such as single-mode operation by small ridge widths of only a few microns, or frequency selectivity by a distributed feedback laser design.

Horizontally emitting lasers within this work are solely broad-waveguide edge-emitters. This simple variant requires a minimized processing effort, enabling fast laser parameter feedback times ( $<2$ days) for the development of epitaxial processes as detailed in section A.5.1. However, these lasers are multimode devices that are not optimized for highest performance, but instead offer a valuable assessment of the epitaxial process quality. By cleaving the laser stripes as the last step of processing, different cavity lengths can be easily created in order to assess relevant characteristic laser parameters.

\subsubsection{Surface-emitting lasers}

In contrast to horizontally-emitting devices, all vertical-emitting designs require additional mirrors which are either epitaxially grown or externally mounted, or are added during processing. The biggest advantage to the vertical concepts is the possibility of a circular geometry, enabling a higher laser beam quality due to the resulting circular beam shape. 


\section{EOM VCSEL}

The very short cavity length of a VCSEL $(<1 \mu \mathrm{m})$ limits the modal gain and causes the mirror losses $\alpha_{\text {mirror }}$ to be the dominant optical loss factor (cf. equation 2.3) for the VCSEL concept. Consequently, VCSELs require mirror reflectivity from both facets well above $99 \%$, to enable reasonable threshold current-density levels. Such reflectivity levels are mostly realized by thick distributed Bragg reflectors (DBRs) with at least 20 pairs in the AlAs/GaAs system. Optical confinement for VCSEL is achieved by etching a circular mesa into the epitaxial structure to enable index guiding and charge-carrier confinement. In order to improve laser characteristics, an oxide aperture layer is often added to confine the applied current to the mesa center, to reduce leakage currents, and for mode selection. As electrically pumped VCSEL use, in most cases, a circular top contact surrounding the output mirror, the maximum output power is limited to milliwatt levels by the area, which can be homogeneously pumped. As soon as contacts are processed VCSELs can easily be tested on wafer without any device separation, due to the fact that light is emitted from the surface.

In this work a more complex variant of the VCSEL is realized, the EOM VCSEL, which includes a monolithically integrated modulator within a second cavity. To access the modulator section, conventional VCSEL processing is altered to realize a third circular middle contact. The same restrictions and advantages as those mentioned above apply to the EOM VCSEL design.

\section{VECSEL}

In contrast to all other laser designs within this work, VECSEL are usually optically pumped, and no lateral patterning or processing of the wafer is applied to the structure post-growth. Instead, the optically-irradiated area defines the actively pumped region of the laser, thus confining the charge-carrier generation to an almost circular spot. For optimum performance, this pump spot should match the focus diameter of the external mirror, which defines the diameter of the laser within the gain chip. In comparison to VCSEL designs, this VECSEL diameter can be much larger - up to hundreds of microns - and the active part of the cavity can be extended to more than a micron, depending on the pump laser absorption characteristics. In consequence, VECSELs enable very high output-power levels of up to several watts in CW mode. VECSEL limitations are given by the almost non-existent lateral confinement and the complex setup, requiring an external pump laser and optical alignment upon mounting the gain chip to an elaborate heat sink. In contrast to other monolithic designs, this external cavity design enables easy intra-cavity access e.g. for efficient frequency doubling. Thus, complete VECSELs are much larger than edge-emitters or VCSELs but offer flexibility, high brilliance and high power. 



\section{Chapter 3}

\section{Experimental}

Metal Organic Vapor Phase Epitaxy (MOVPE) is used within this work for the fabrication of all samples. Advanced designs require the fabrication of numerous calibration and optimization test samples in order to assess distinct material parameters and nanostructure properties preceding the final MOVPE device growth process itself. Particularly, active layers as QDs require extensive research to elaborate optimal growth parameters. The key characterization methods and equipment, as well as test-structure designs employed are briefly described within this chapter.

\subsection{Metal Organic Vapor Phase Epitaxy}

Commercial MOVPE systems have been available since the 1980s, and are now well established as the world's leading manufacturing technology for semiconductor nanostructures. The fundamental concept is based on the decomposition of metal-organic or hydride precursors in vapor-phase within a reactor containing substrates (wafers). Group III and group $\mathrm{V}$ material flows are computer-controlled and piped separately into the reactor to eliminate premature chemical reactions. Decomposition of precursor molecules within the reactor involves catalytic and pyrolytic processes in the gas phase and on surfaces. Adsorbed atoms and molecules diffuse on the substrate surface, and are subsequently integrated into the semiconductor surface, resulting in precisely controlled epitaxial mono-crystalline layer growth. Epitaxial layer thicknesses range from a monolayer to several microns and are reproducible with an accuracy of less than one nanometer, if growth parameters are properly calibrated and controlled. Nowadays large-scale manufacturing systems offer capacities of 16x4" or 69x2" substrates simultaneously. MOVPE principles and physics are described in more detail by Stringfellow [Str99].

Epitaxial growth of all samples in this work is carried out with a commercially available Aixtron 200/4 horizontal flow quartz glass research reactor system. Possible commercial reactor configurations range from a single quarter of a 2 " wafer up to $3 \times 2$ " or 4 " substrates. Heating up to $\approx 800^{\circ} \mathrm{C}$ is realized by a $1200 \mathrm{~W}$ infrared heater which is replaced during this work by a custom-built version with improved mirror geometries allowing for $\approx 25 \%$ faster heating times. As heating is indirect, through absorption of incident infrared light by a carbon susceptor-containing thermocouples, and the substrate is positioned on a separate 
gas-foil rotation wafer mount, all stated temperatures need to be adjusted by the significant thermal offset of the setup. This offset depends on several parameters such as reactor configuration, total reactor flow, substrate type and size, and reactor wall coating.

All stated temperatures overestimate the real wafer surface temperature by $\approx 20^{\circ} \mathrm{C}$ !

Temperature offset calibration is performed at $577^{\circ} \mathrm{C}$ using aluminum-coated eutectic silicon substrates as detailed in Section 3.1.2. The effects of substrate size on temperature offset are detailed in Section 5.2.2. Only commercially available epi-ready 2" GaAs $\left(\begin{array}{lll}1 & 0 & 0\end{array}\right)$ substrates with $400 \pm 20 \mu \mathrm{m}$ thickness and no off-cut are used without any pre-epitaxial preparation. Intentionally doped $\mathrm{N}^{+}$-substrates use silicon as donator for free charge carrier concentrations of $n \approx 3 \cdot 10^{18}$. The whole MOVPE system is modified multiple times for improvements, maintenance or to meet requirements of research projects. The MOVPE precursors of the system used during this work are:

\section{Group III}

$\begin{array}{lll}\text { trimethylgallium (TMGa) } & \mathrm{Ga}\left(\mathrm{CH}_{3}\right)_{3} & 2 \text { lines available } \\ \text { trimethylaluminium (TMAl) } & \mathrm{Al}\left(\mathrm{CH}_{3}\right)_{3} & 1 \text { line }+2^{\text {nd }} \text { line added recently } \\ \text { trimethylindium (TMIn) } & \mathrm{In}\left(\mathrm{CH}_{3}\right)_{3} & 2 \text { lines available } \\ \text { Group V } & & \\ \text { tertiarybutylarsine (TBAs) } & \mathrm{AsH}_{2} \mathrm{C}\left(\mathrm{CH}_{3}\right)_{3} & 2 \text { lines available } \\ \text { arsine } & \mathrm{AsH}_{3} & 1 \text { line, added during this work } \\ \text { tertiarybutylphophine (TBP) } & \mathrm{PH}_{2} \mathrm{C}\left(\mathrm{CH}_{3}\right)_{3} & 1 \text { line available } \\ \text { Dopants (one line each) } & & \\ \text { diethyltellurium (DETe) } & \mathrm{Te}\left(\mathrm{CH}_{2} \mathrm{CH}_{3}\right)_{2} & \text { n-dopant } \\ \text { silane in } \mathrm{H}_{2}(1 \% \text { or } 0.02 \%) & \mathrm{SiH}_{4} & \text { n-dopant, added during this work } \\ \text { carbon tetrabromide } & \mathrm{CBr}_{4} & \text { p-dopant } \\ \text { dimethylzinc (DMZn) } & \mathrm{Zn}\left(\mathrm{CH}_{3}\right)_{2} & \text { p-dopant }\end{array}$

The carrier gas used for all samples is Pd-diffused $\mathrm{H}_{2}$. For the most recent samples the Pd-cell purification system is replaced by an equivalent filter-based solution. The quartz-glass reactor is equipped with a separately purged transparent viewport opening, providing access for optical characterization of the wafer surface during growth, as detailed in A.1.1. Programming of all processes is based on commercial Aixtron CACE software. For debugging and growth parameter calculations, as well as for characterization results analyses, several custom-made software tools were programmed as detailed in A.1.2.

\subsubsection{QD growth process}

As the optimization of QD growth processes was extensively studied within this work, the fundamental QD growth process is discussed in detail here. The employed QD growth process includes several crucial steps, which significantly influence final QD properties. 
Impact of individual growth parameter changes are discussed in detail in Chapter 4 Table 3.1 depicts all fundamental steps of the QD growth process, including growth interruptions (GRI) and temperature ramps.

\begin{tabular}{lllrl}
$\#$ & Growth & Description & Temperature & Comment \\
\hline \hline 1. & GRI & cool down to $\mathrm{T}_{Q D}$ & to $\approx 515^{\circ} \mathrm{C}$ & $\begin{array}{l}\text { temperature stabilization, } \\
\text { arsenic conditioning }\end{array}$ \\
\hline 2. & In $(\mathrm{Ga}) \mathrm{As}$ & QD deposition & $515^{\circ} \mathrm{C}$ & $\begin{array}{l}\approx 1.7 \mathrm{ML} \text { In } A s \text { for } \\
\text { 2D }-3 \mathrm{D} \text { transition required }\end{array}$ \\
\hline 3. & GRI & QD formation & $515^{\circ} \mathrm{C}$ & $\begin{array}{l}\text { optional, without } \\
\text { arsenic stabilization }\end{array}$ \\
\hline 4. & In GaAs & strain reducing & $515^{\circ} \mathrm{C}$ & optional, $\leq 20 \%$ indium \\
& layer (SRL) & & \\
\hline 5. & GaAs & low temperature & $515^{\circ} \mathrm{C}$ & prevents indium loss \\
& & capping layer & & during heating \\
\hline 6. & GRI $(G a A s)$ & heat up to $\mathrm{T}_{\text {spacer }}$ & to $\approx 615^{\circ} \mathrm{C}$ & $\begin{array}{l}\text { growth during heating } \\
\text { is optional }\end{array}$ \\
\hline 7. & GaAs & spacer & & growth rate up to $2 \mu \mathrm{m} / \mathrm{h}$ \\
\hline 8. & GRI & annealing & $615^{\circ} \mathrm{C}$ & optional
\end{tabular}

Table 3.1: Employed steps of the QD growth process are listed. This fundamental sequence is used for all QD growth processes with individually tuned parameters as described in Chapter 4

A prerequisite for reproducible QD properties is a defect-free crystal surface with a welldefined arsenic partial pressure and stable temperature conditions within the reactor. Thus, all preceding growth steps need to achieve the very same wafer surface conditions for each QD layer. As shown in table 3.1 the first step is a GRI in order to condition reactor and wafer surface properties. As a result the temperature becomes stable and the arsenic saturation of surfaces inside the reactor is set. The employed QD material deposition speed is $\approx 0.5$ $\mu \mathrm{m} / \mathrm{h}$, which is rather high when compared to other reported QD growth processes [Hei02, Gui06b]. As 2-D to 3-D transition occurs immediately after reaching the critical layer thickness, QD formation happens in parallel with the QD material deposition. Information on the critical layer thickness of $\approx 1.7 \mathrm{ML}$ InAs is detailed within the dissertation of Frank Heinrichsdorff [Hei98a] and in [Leo94, Kro96b, Bim98]. The process presented results in a short QD deposition time of only a few seconds, aiming at a decoupling of material deposition time and subsequent GRI for QD formation. This optional GRI directly subsequent to the QD material deposition is used as a tuning parameter to produce larger QDs with a red-shifted spectral emission (cf. Figure 4.3). Further spectral red-shifting can be realized by an optional SRL, which reduces the lattice mismatch offset to the capping 
material, thus reducing the strain within the QDs (cf. Figure 4.4). Growth of the first covering layer on top of the QDs stops material redistribution between QDs of different size, thus QD size increase is halted. In parallel, this first covering layer on top of the QDs strongly influences QD shape evolution leading to the final QD geometry within the crystal [Son03, Cos06]. Consequently, the SRL step provides the strongest influence on final QD properties, depending on its composition and growth parameters (cf. Figure 4.5). Capping of the QD layer with GaAs before increasing temperature again is essential for preventing indium flushing at higher temperatures [Sas07, Kum11]. As this capping layer is grown at the same low temperature as the QDs themselves, it exhibits inferior material quality which likely contains a higher density of point defects than $\mathrm{GaAs}$ fabricated at the optimum temperature. As detailed in 4.1.2, such imperfect material promotes a spectral blue-shift of QD emission. To reduce defect densities in the proximity of active QD layers, the low-temperature capping layer needs to be as thin as possible but as thick as necessary to prevent QD degradation during heating. Subsequent to heating, spacer material is grown at a rather high growth rate to minimize the overgrowth time of buried QD layers. A final annealing GRI step can be used to heal out defects and to smooth the surface, but it is not obligatory if the QDs already show temperature stable properties and a good stacking behavior [Kai06, Yan06]. These QD growth process steps can be repeated to generate stacked QD structures. For stacking, a sufficiently thick high-quality spacer layer is required to achieve the same flat, strain and defect-free crystal surface as that for the initial layer. This ensures stable QD properties for subsequent QD layers.

\subsubsection{Wafer temperature calibration}

Reflectivity of silicon-aluminum eutectic wafers was monitored in-situ during heating to measure the $577^{\circ} \mathrm{C}$ eutectic transition point. For highest accuracy, heating speed was reduced to $\leq 0.1 \mathrm{~K} / \mathrm{s}$. Data to calculate the temperature offset was taken at $50 \%$ of initial reflectivity. Measurements with partially aluminum-coated eutectic substrates (circular coating at the wafer center $\approx 10 \mathrm{~mm}$ in diameter) show more than a $5 \mathrm{~K}$ increase in transition point temperature. This effect can be explained by Kirchhoff's law of thermal radiation as detailed in Section 5.2.2. The temperature offsets measured can additionally vary by $\approx 10$ degrees, depending on reactor configurations. Thus, results from temperature calibrations can offer only an approximation for the absolute wafer temperature. Regarding epitaxy, such temperature calibrations are essential for the identification of changes after maintenance work or configuration changes. 


\subsection{Optical characterization}

Optical measurement methods are standard for the vast majority of samples. Steady-state PL measurements are used as a principal tool to characterize the quality and properties of optically-active nanostructures, whereas surface reflectivity data is used to assess passive device-component parameters such as DBR or cavity position. An overview of the additional characterization setups employed within this work to analyze specific material characteristics is given within the appendix A.2. Detailed methods, equipment types, and data analysis can be found therein.

\section{Photoluminescence}

For RT measurements, samples were mounted to a metal heat-sink employing conductive silver. A closed-cycle helium-cryostat was used for temperature-dependent measurements $(\mathrm{T}=10 \mathrm{~K}-360 \mathrm{~K})$. Optical pumping was performed by a frequency doubled $\mathrm{Nd}: \mathrm{YVO}_{4}$ laser at $532 \mathrm{~nm}$ with $0.5 \mathrm{~W}$ output power. A prism within the optical path filters fundamental infrared laser emission. Diameter of the focused spot on the sample is $\approx 50 \mu \mathrm{m}$. A set of neutral density filters enable excitation densities of $0.5 \mathrm{~W} / \mathrm{cm}^{2}$ - OD4, $5 \mathrm{~W} / \mathrm{cm}^{2}$ - OD3, $50 \mathrm{~W} / \mathrm{cm}^{2}$ - OD2, $500 \mathrm{~W} / \mathrm{cm}^{2}$ - OD1 and $5 \mathrm{~kW} / \mathrm{cm}^{2}$ - OD0. Spectral filtering on the detection side was carried out by a $50 \mathrm{~cm}$ focal length monochromator equipped with a single 600 lines $/ \mathrm{mm}, 1 \mu \mathrm{m}$ blaze grating, yielding a linear dispersion of $\approx 3.3 \mathrm{~nm} / \mathrm{mm}$. A Northcoast liquid nitrogen cooled germanium pin-diode detector, coupled to a lock-in amplifier setup was used for detection.

RT measurements for all optically active samples were carried out at low and at high excitation densities to identify ground state (GS) luminescence and the convolution of excited states (ES), wetting layer (WL), and substrate luminescence separately. However, most samples were measured from OD3 to OD0 using an automated setup routine to monitor the onset of ES luminescence. To verify that OD3 excitation predominantly shows GS QD emission, an assessment of the mean exciton population of a QD is conducted.

First, the absorption for a standard PL test sample, as detailed in 3.3, with a single active layer is calculated. Refractive indices and extinction coefficients are taken from Aspnes et al. [Asp86]. Reflected intensity is calculated to $R \approx 30 \%$ using the transfer matrix method (cf. A.4) and material data for the pump laser wavelength at $532 \mathrm{~nm}$. Absorption within the $5 \mathrm{~nm} G a A s$ capping layer, the $25 \mathrm{~nm} A l_{0.7} G a_{0.3} A s$ top barrier layer, and other losses are neglected for this upper limit approximation. Absorption within the $300 \mathrm{~nm}$ thick GaAs matrix is calculated to $90.9 \%$ of the available light using equation 6.2 and an absorption coefficient of $\alpha \approx 8 \mu \mathrm{m}^{-1}$. Thus $3.18 \mathrm{~W}$ of the incident pump light are converted to about $8.5 \cdot 10^{18} s^{-1}$ electron hole pairs. 


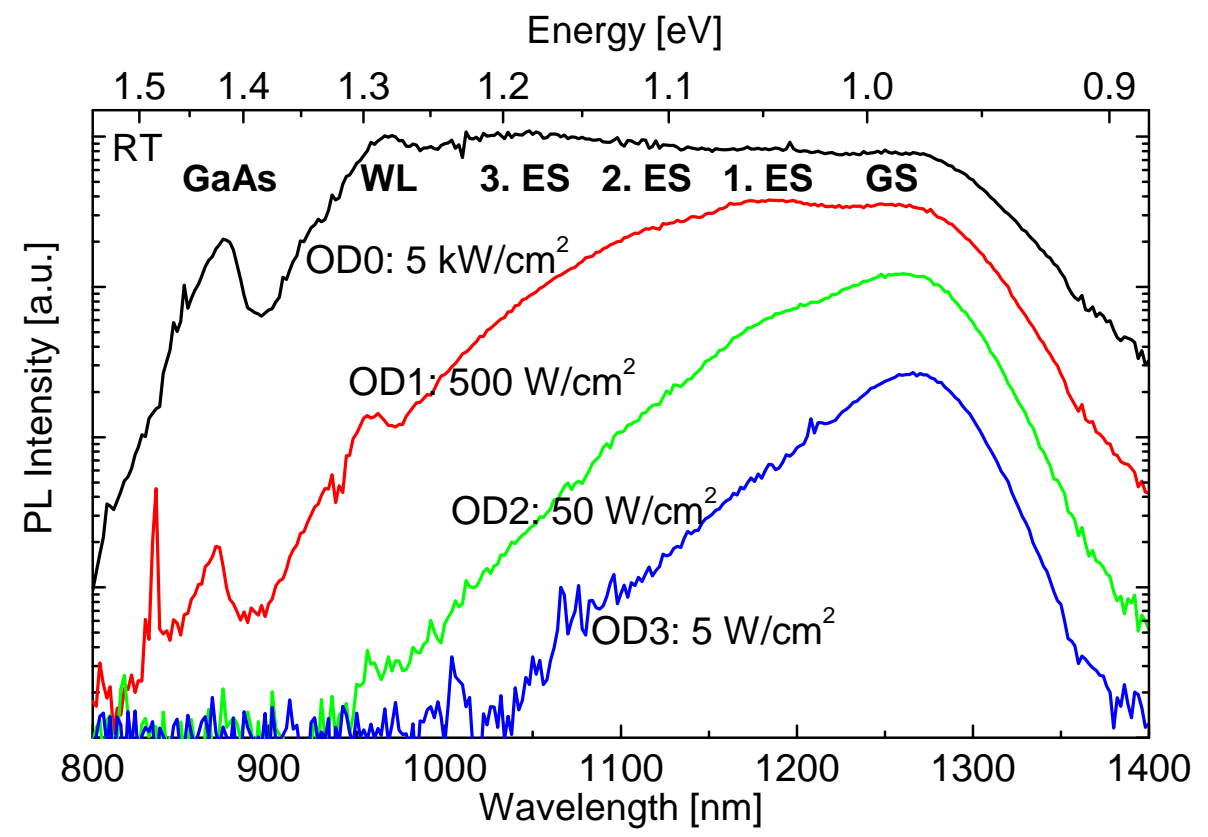

Figure 3.1: PL test sample with a single QD layer measured at RT at all four excitation densities. The onsets of multiple ES, WL, and GaAs luminescence become visible at higher excitation levels.

Secondly, the available electron-hole pairs are set in relation with the spontaneous single exciton recombination time and the QD density. Given a moderate QD density of $1 \cdot 10^{10} \mathrm{~cm}^{-2}$, the maximum number of supplied electron-hole pairs per QD is estimated to be $8.5 \cdot 10^{8} s^{-1}$. As the GS single exciton lifetime has been reported to be about $\tau_{\text {exciton }} \approx 1 \cdot 10^{-9} s$, on average less than one exciton populates a QD [Hei97c, Feu08]. Consequently, QD GS emission is dominant for the assumed QD density and photon lifetime. It should be noted, however, that these two values depend strongly on QD properties such as QD size. Nevertheless, it can be concluded that if ES emission dominates already at OD3, the QD density has to be in the range of $10^{9} \mathrm{~cm}^{-2}$ or lower. In contrast, for a good quality QD layer with a higher QD density of $4 \cdot 10^{10} \mathrm{~cm}^{-2}$ or more, OD2 excitation will also mainly show GS luminescence, as the GS will be occupied by an additional biexciton exhibiting an almost halved $\tau_{\text {exciton }}$ [Feu08]. At OD1, ES QD luminescence becomes clearly visible for all QD samples, and higher order ES and WL can emerge. For the even higher OD0 excitation density, a convolution of GS, all ES, WL, and substrate luminescence is measured. Figure 3.1 shows exemplary RT PL measurements at all four excitation densities of a PL test sample with a single QD layer. For these QDs close to $1.3 \mu \mathrm{m}$ the first ES already emerges at OD2, while GS luminescence still dominates. OD1 and OD0 data shows the onset of higher order ES, as well as WL and GaAs matrix emission. 
Thus, PL characterization measurements are a versatile and fast tool to assess QD properties in terms of spectral width and position, as well as the approximate QD density. In contrast to the microscopic determination of QD densities, this method is solely based on optically-active QDs. Thus information about non-radiative defects can be deduced from low excitation data, whereas maximum optical gain from samples can be concluded from high excitation measurements. The density of optically-active QDs can be deduced from the ES state onset. To enable comparable results independently of the active layer type or number, standardized PL test sample structures were used (cf. Section 3.3).

Further information on semiconductor optics and spectroscopy can be found in the textbook of C. F. Klingshirn [Kli07]. Details on QD spectroscopy are published by U. W. Pohl et al. [Poh08b].

\section{Reflectometry}

Surface reflectivity measurements were used for DBR and cavity length calibrations of test structures for vertical emitting devices. An optical spectrum analyzer and a white light source were both coupled via a 50/50 splitter to the same optical fiber. The apex of the optical fiber was equipped with a micro lens to focus incident light on the wafer and to capture reflected light from the surface. Measurements were calibrated with a gold-coated sample as a $100 \%$ standard. Gold offers the most efficient reflective coating for the infrared wavelength range with a stable absolute reflectance of $>98 \%$ between 1.0 and $1.3 \mu \mathrm{m}$, whereas towards $0.8 \mu \mathrm{m}$ reflectance decreases to $\approx 94 \%$ (values are for vertical incidence).

\subsection{Sample structure design}

For calibration of individual parts of complex device structures, convenient test sample designs are required. Often individual parts or smaller versions of the final device design are used for this purpose. Thus, separate characterization and tuning of required properties such as spectral position of mirror reflectivity, cavity resonance, and active layer emission or charge carrier densities becomes possible. Additionally, sample structures are essential for MOVPE growth parameter calibrations such as growth rates and material compositions, and enable the identification of error sources causing defects and surface roughness. All test sample structures need to be able to be grown quickly for optimization iterations, and to verify the desired design parameters without the influence of other parts of the final design. The most important test sample design is the PL test structure, which is discussed in the following subsection. All designs for other characterization methods are detailed within the appendix in A.3. 


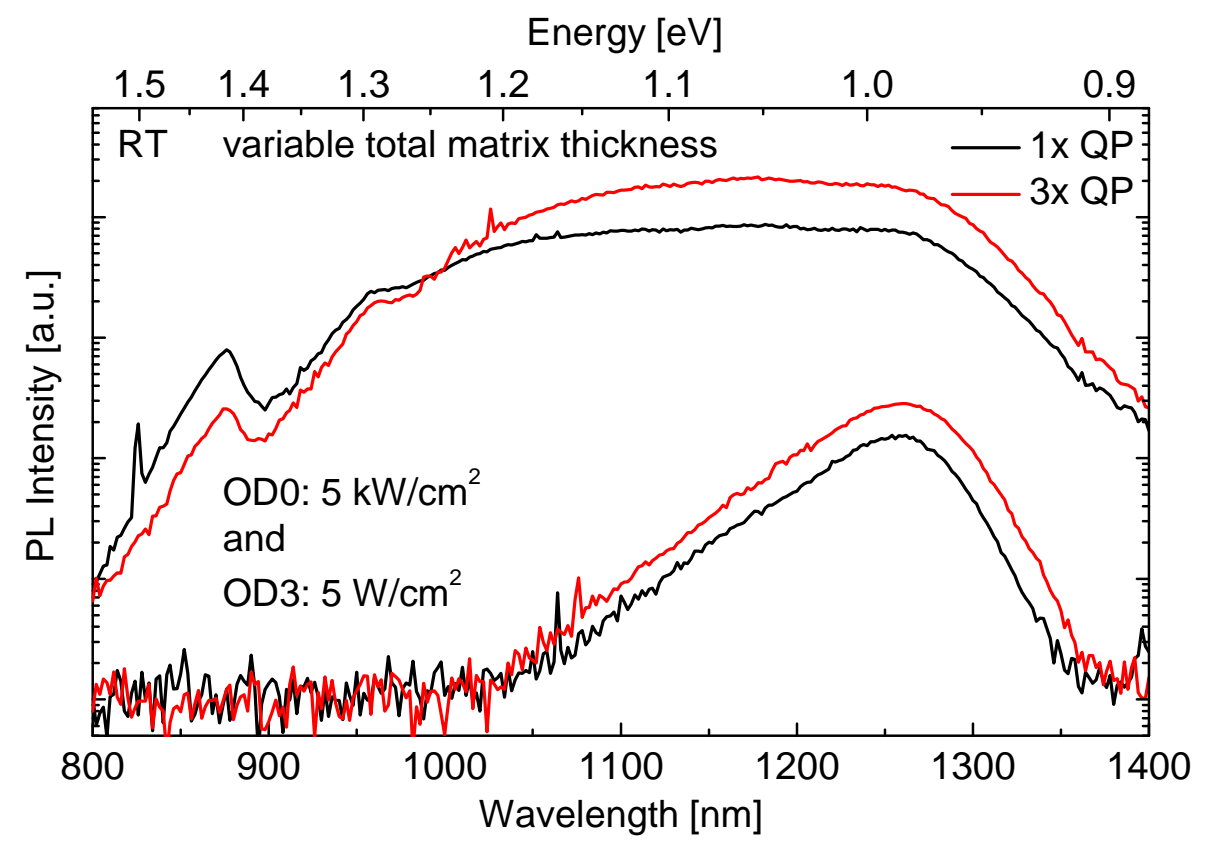

Figure 3.2: Spacer thickness between barriers and first/last QD layer is constant, but total matrix thickness varies by stacking of QD layers. OD3 PL increases due to the increased total absorber volume available for charge carrier generation.

\section{PL test structures}

The goal of the test structure design for PL measurements is to allow for direct comparison of results independently of active medium type or the number of active layers within the structure. For simple PL measurements it is sufficient to integrate an active layer into an absorbing matrix crystal. This allows for the generation of charge carriers, which can diffuse to the active layer and relax into the energetically lower confined states. As the amount of charge carriers available to the active medium depends directly on the absorbed pump light within the diffusion length from the active layers, equaling several microns in undoped GaAs [Cas73], a well-defined absorber volume is required for comparability between samples. This is ensured by two $A l G a A s$ charge carrier barriers bordering the absorbing $\mathrm{GaAs}$ matrix material containing all active layers. If the distance between these AlGaAs barriers depends on active layer stacking height, absorption properties are altered, thus direct PL comparisons are difficult. Regarding low excitation measurements (OD3), which do not saturate QD GS emission as calculated in Section 3.2, this becomes especially prominent. Figure 3.2 shows this significant effect. Here OD3 luminescence increase must be attributed to the sample thickness increase, as the single QD layer does not saturate and is able to collect all available charge carriers. PL test samples from this work feature active layers embedded in a standardized $300 \mathrm{~nm}$ thick GaAs matrix core. Thus, low excitation 


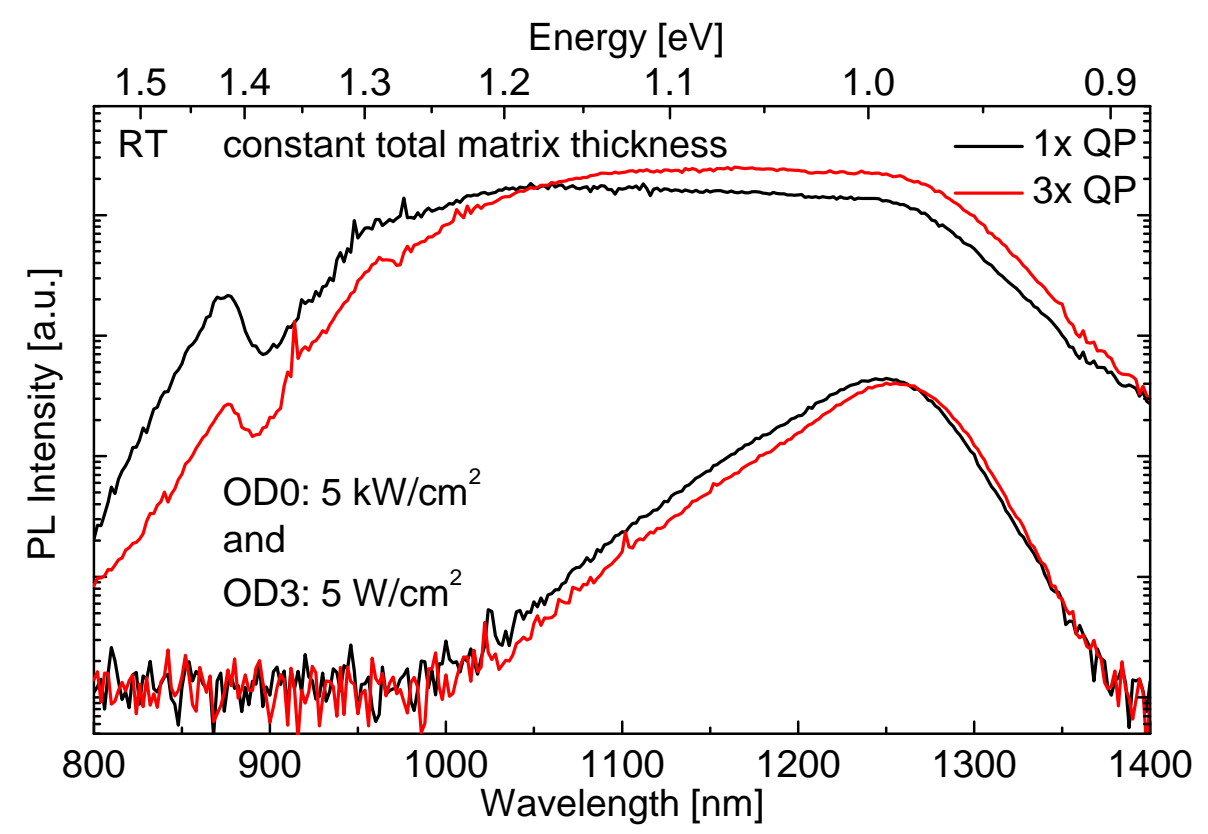

Figure 3.3: Total matrix thickness is kept constant to $300 \mathrm{~nm}$ independently on the number of QD layers. OD3 PL is almost constant while the OD0 PL increases due to the 3-fold stacking of QD layers.

OD3 measurements of samples containing differing numbers of active layers show identical results (cf. 3.3. If such OD3 measurements differ upon stacking, an increased number of non-radiative defects within the active layer stack can be concluded. The constant absorber thickness design for PL test samples enables efficient stacking optimization, and helps to identify and to eliminate defect sources from the QD growth process. For high excitation (OD0), this constant matrix sample design enables estimations of the increase in ground state gain achieved by stacking active layers. 



\section{Chapter 4}

\section{MOVPE processes}

This chapter discusses the influence of individual QD growth parameters and stacking challenges, along with the material quality and doping levels used for laser device growth. For these investigations numerous test samples are grown on a commercially available Aixtron 200/4 MOVPE system. Thus all processes are transferable to industrial mass production systems. The main difference between the employed system and industry production systems is the horizontal flow 3 times 2 inch reactor, versus large multi-wafer reactors for growth on dozens of wafers simultaneously. For economic reasons, all samples of this work are grown with a reactor configuration for either a single 2 inch or a quarter piece of a 2 inch wafer. Further details of the employed MOVPE system are outlined in 3.1

\subsection{Quantum dot growth}

QD growth optimization for laser applications aims at a maximized optical gain for every individual QD layer. Additionally, stacking optimization is required to generate uniform QD properties across all active layers. In contrast to QW growth, QD epitaxy needs to deal with a non-uniform lateral strain distribution due to the three-dimensional character of the QDs. In particular, the overgrowth of QD layers requires extensive optimization to achieve a perfectly flat surface for all subsequent layers. Details of the basic steps of the employed QD growth process sequence are given in 3.1 .1

\subsubsection{QD optimization close to $1300 \mathrm{~nm}$}

InGaAs or InAs QDs in a GaAs matrix with sufficient gain for laser applications are easily tunable within a spectral emission range from $1050 \mathrm{~nm}$ to $1200 \mathrm{~nm}$. To achieve QD emission close to $1300 \mathrm{~nm}$ with high QD areal density, a precise tuning of multiple growth parameters is needed to create a sufficiently large red shift. Achieving QD emission beyond $1300 \mathrm{~nm}$ is possible even with $\operatorname{In} G a A s$ QDs in a $G a A s$ matrix without any special capping layer, but is linked to a strong reduction in luminescence due to decreased QD density and increased defect formation [Pas04]. The crucial point, however, is to grow long wavelength QD layers closely stacked and with high enough optical quality to generate sufficient gain for laser devices. To assess the optical quality of the QDs and the surrounding matrix 


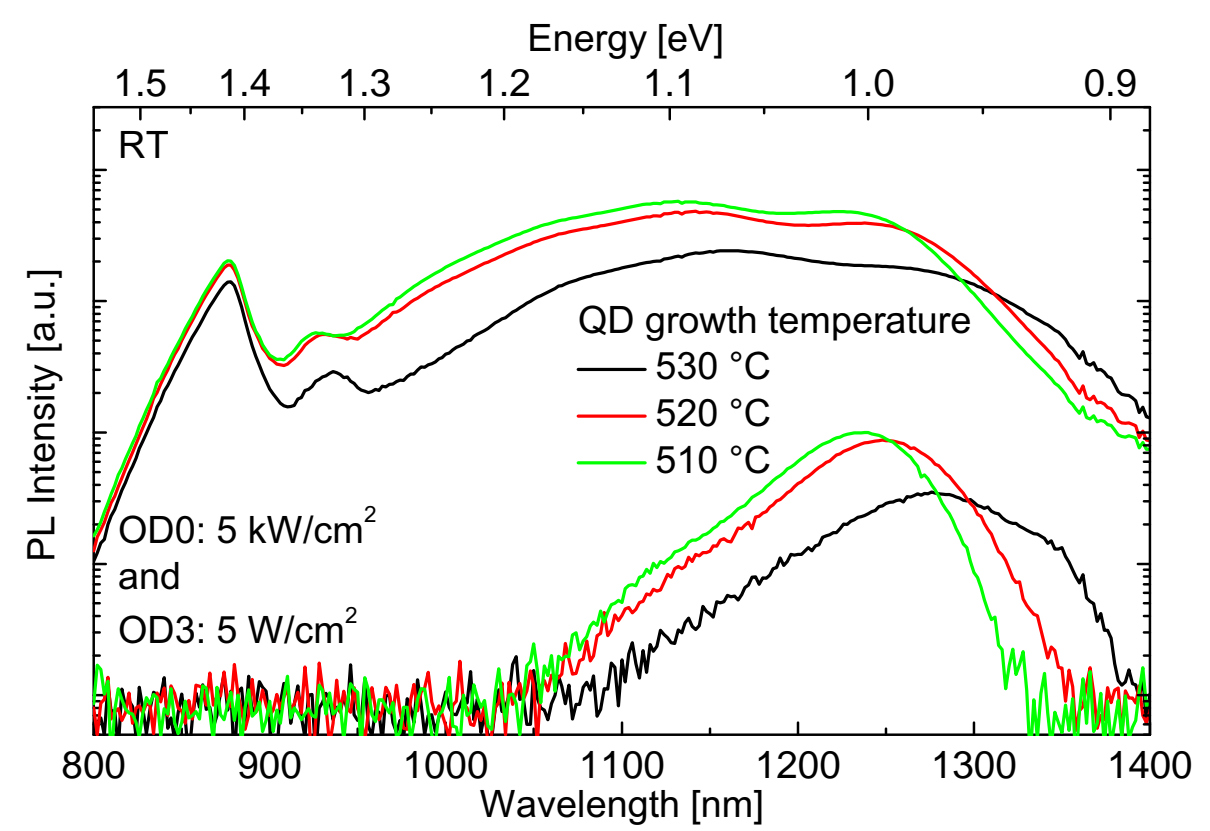

Figure 4.1: PL measurements of test samples with single $I n_{0.65} G a_{0.35} A s$ QD layer without SRL. The variation of the QD deposition temperature shows a PL increase for lower growth temperatures due to higher QD densities. Decreased PL associated with a strong red shift for higher temperatures indicates larger QDs and starting cluster formation.

material, standardized PL test samples are grown (cf. 3.3) and characterized using room temperature PL (cf. 3.2). The GaAs matrix thickness of these PL test structures is already set to the final waveguide thickness of the target laser structure. To investigate the influence of individual parameters usually only a single parameter is changed from run-to-run for the QD optimization process.

\section{QD growth temperature}

Early investigations of the QD formation process showed that higher growth temperatures lead to an increased QD base size, while simultaneously decreasing the QD density [Osh94]. Additionally, an increase in cluster formation with increased temperature was reported by Steinmetz et al. [Ste97]. Both phenomena are explained by the increased adatom diffusion, which is described by $D \propto \exp \left(-E_{D} / k T\right)$ with the activation energy $E_{D}$ for surface diffusion. Kinetic nucleation models show the influence of diffusivity on the QD density $N$ as $N \propto 1 / D$ [Ven84]. Thus the QD growth temperature can assist in creating lower QD densities for single QD applications, or to create larger QDs for long wavelength devices. This correlation can clearly be evidenced by PL measurements, as shown in Figure 4.1 . The change of the nominal reactor temperature from $530^{\circ} \mathrm{C}$ to $510^{\circ} \mathrm{C}$ during deposition 


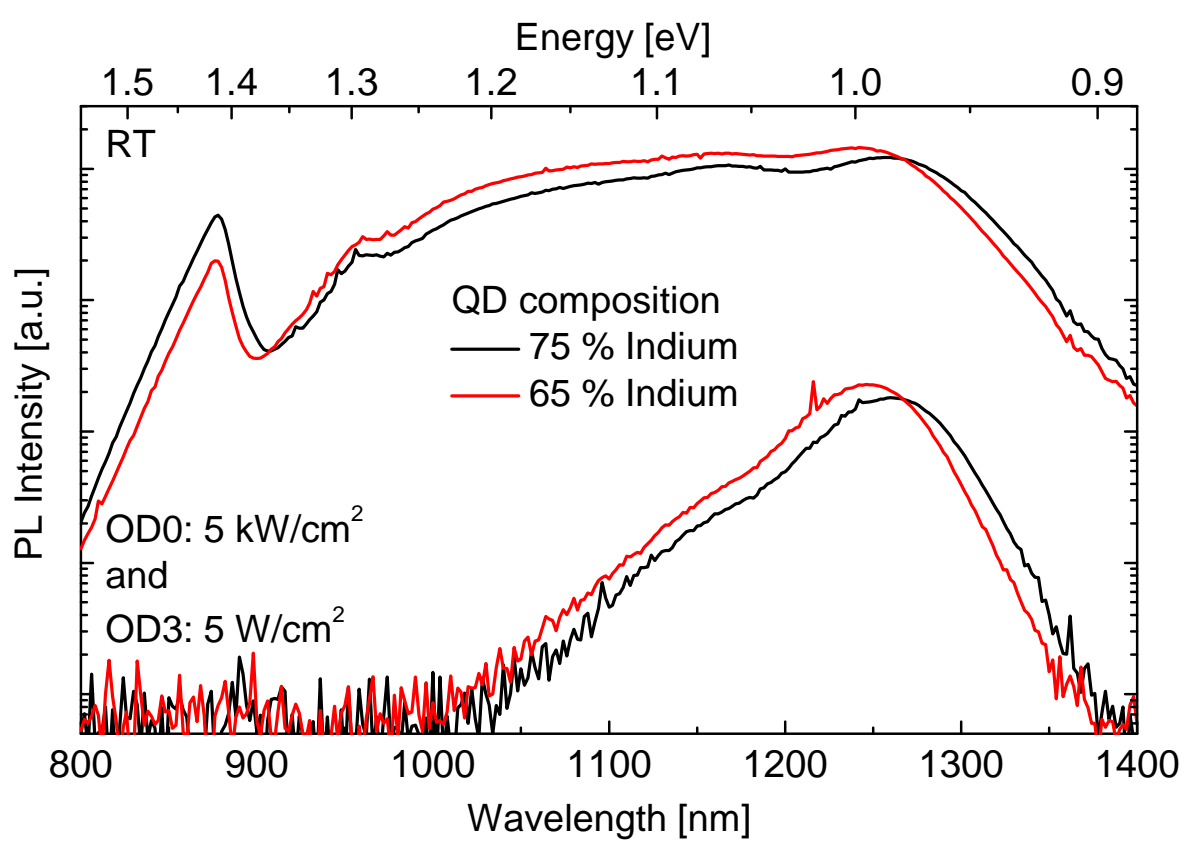

Figure 4.2: Single QD layer test samples with $I n_{x} G a_{1-x} A s$ QDs with $\mathrm{x}=0.65$ and 0.75 and a strain reducing $I n_{0.08} G a_{0.92} A s$ layer. The peak emission wavelength shifts $15 \mathrm{~nm}$ with increased indium content, while the luminescence decreases by $12 \%$.

and GRI of the QDs results in a $40 \mathrm{~nm}$ blue shift accompanied by a three-fold increase in intensity, which can be attributed to the formation of smaller QDs with a higher areal density. Regarding the significant spectral broadening and intensity loss from $520^{\circ} \mathrm{C}$ to $530^{\circ} \mathrm{C}$, it can be assumed that this corresponds to the start of dislocation cluster formation. Nevertheless, changes in growth temperature are not trivial due to cross influence on growth rate/thickness and V/III ratio, thus growth temperature changes necessitate subsequent careful parameter changes.

\section{QD composition}

The nominal composition of the QD material itself has only a minor influence on the final QD composition and on the maximal QD emission wavelength (cf. Figures 4.2 and 5.14). This can be explained by indium redistribution during the QD formation process. While the indium concentration within the QDs increases, the surrounding material exhibits a reduced indium concentration [Wal01]. Thus, long wavelength emission around $1300 \mathrm{~nm}$ has been achieved with pure $I n A s$ QDs as well as with $I n_{0.5} G a_{0.5} A s$ QDs [Muk94, Tat01]. Much more importantly is the QD capping process, which defines the final properties of the QDs within the matrix crystal [Gon04, Cos06]. This can be explained by indium-gallium phase separation during overgrowth with an In GaAs alloy, and by intermixing processes during 


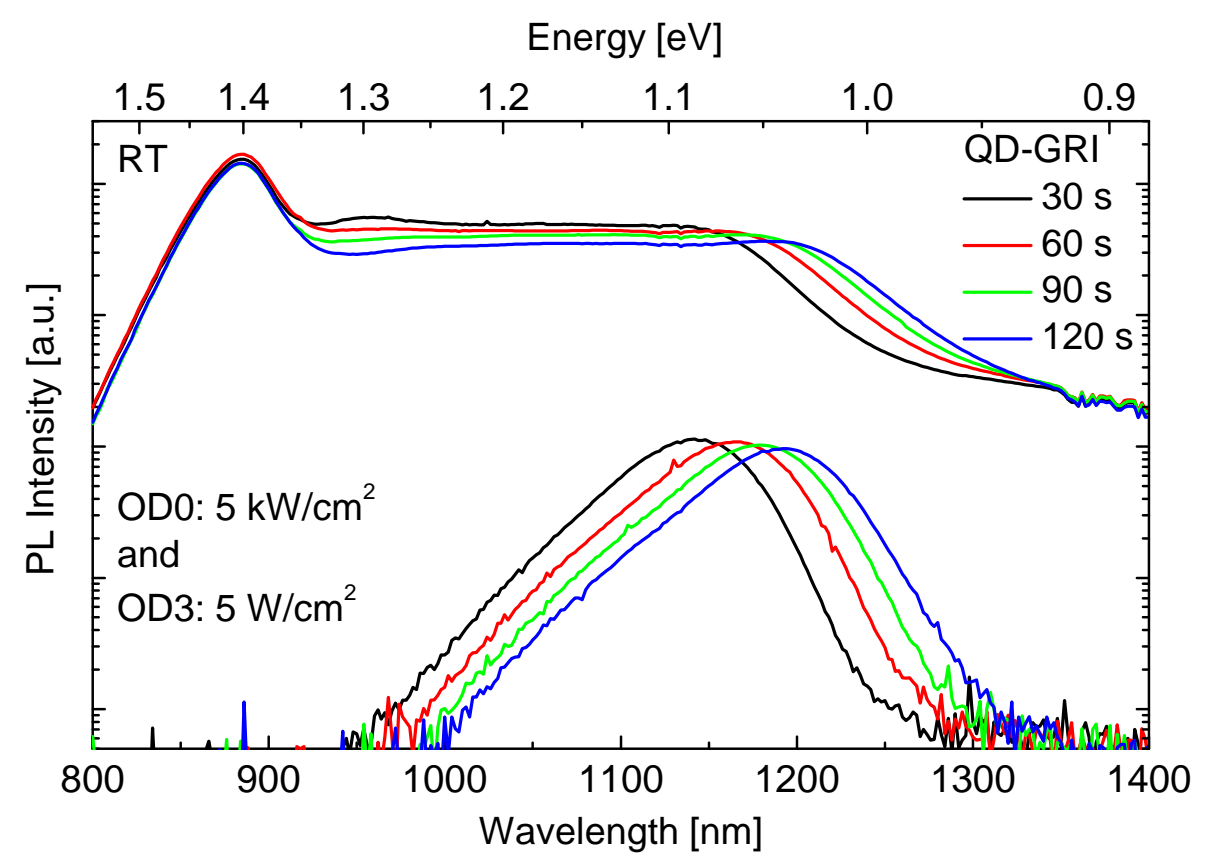

Figure 4.3: Influence of the GRI subsequent to the In $G a A s$-QD material deposition is investigated. The GRI is carried out without arsenic surface stabilization before covering the QDs with a GaAs capping layer. Red shifts with increasing GRI are measured to 22, 16, and $12 \mathrm{~nm}$ with almost constant OD3 peak luminescence.

the capping [Max00, How05]. These effects lead to indium accumulation within QDs, and QD compositions can differ significantly, depending on growth parameters, from the initial nominal material compositions [Len02, Lem04, Bim08]. For experiments contained within this work, In GaAs QDs with $65 \%$ or $75 \%$ indium are employed for all samples instead of pure InAs QDs, due to better control of the QD growth process with this ternary material. This enables more control over the QD ripening process using a long GRI step (2 to $3 \mathrm{~min}$ ), especially for QDs emitting close to $1.3 \mu \mathrm{m}$. In contrast, InAs QDs for long wavelength emission require adapted growth process parameters which often rely on very slow QD material growth rates and a short or no subsequent GRI, as QD formation already takes place during the QD material deposition step [Tat01, Par04].

\section{Growth interruption}

By using In GaAs QDs with short deposition times ( $<10 \mathrm{~s})$, the optional GRI directly following the deposition of the QD material becomes more important for the QD formation. This acts as a tuning parameter to control for the evolution of QD properties. The GRI is usually carried out without any arsenic stabilization of the surface to minimize surface mobility of indium adatoms, thus improving control of the QD 2-D to 3-D transition. 
During the GRI, exchange processes of indium and gallium atoms take place within the QD ensemble and the wetting layer (WL). These processes are driven by strain relaxation to minimize the total energy of the QD layer system.

Initially indium accumulates in the developing QDs originating from the grown In $G a A s$ layer. The strain energy within the initially thick InGaAs layer is reduced as transferred indium atoms support the three-dimensional QD formation where strain relaxation can take place by lattice bending. After this redistribution, the indium is distributed between a thin WL and the QDs with maximum indium concentration in the QD apices [Wal01]. This process has been evidenced by several groups with different techniques, e.g. XRD, HRTEM, STM [Kro96b, Wog97, Gra97].

The GRI can be used as an efficient parameter to tune the emission wavelength, while keeping the total composition constant, by employing In GaAs QDs. A longer GRI causes a red shift of the emission wavelength due to the formation of larger QDs, as shown in Figure 4.3. This effect is based on material redistribution between the QDs via the WL and is known as ripening. During this ripening process small QDs are dissolved while larger QDs accumulate material, thus the QD density decreases [Pöt04]. Driven by the need to minimize the total QD layer energy, the QD size increases towards a theoretically predicted stable equilibrium [Mo198, Shc99], although experimentally only metastable QD ensembles are observed. The QD size increase, and corresponding red shift, only works up to a certain limit that depends on the indium amount within the QDs and the growth parameters. If the QDs become too large they start to form dislocation clusters which grow further at the cost of other intact QDs driven by the stronger strain relaxation within these clusters. A strong indication of this point of dislocation cluster formation is a drop in PL luminescence as a consequence of a QD material or GRI increase. For the QD structures presented in this work GRI times ranging from $0 \mathrm{~s}$ to $180 \mathrm{~s}$ were employed, depending on the target wavelength.

\section{Strain reducing layer}

The most important step of the QD growth process is the capping procedure. During capping layer growth the QDs gain their final shape and composition. The resulting QD properties can subsequently only partially be tuned by high temperature annealing [Seg06]. During overgrowth, QD material is redistributed from the top to the sides of the QDs, resulting in reduced QD height and an elongated QD shape with respect to the initial uncapped shape [Son03, Cos06]. By tuning the capping layer growth parameters, QD properties can be changed within a certain range [Pas04]. To tune the emission to longest wavelengths a strain reducing capping layer (SRL) can be used in this step [Ust99, Tat01]. Such a SRL consists of a thin InGaAs QWs with a lower indium concentration than the QD material. Usually less than $20 \%$ indium is used in the SRL, since higher content 


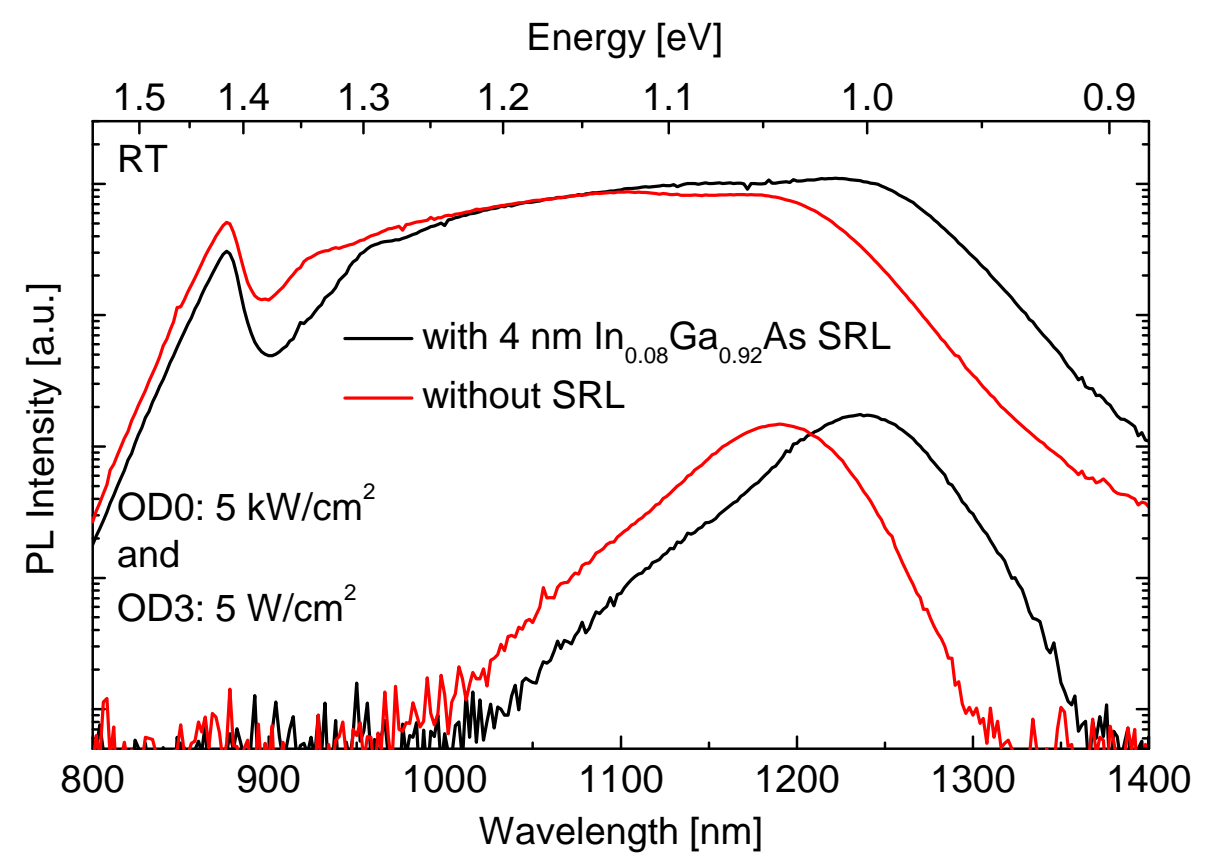

Figure 4.4: Influence of a $4 \mathrm{~nm} \operatorname{In}_{0.08} G a_{0.92} A s$ SRL grown as first capping layer on $\operatorname{In} G a A s$ QDs compared to a QD sample without SRL. The SRL causes a significant red shift from $1190 \mathrm{~nm}$ to $1240 \mathrm{~nm}$ while showing increased luminescence.

results in significantly reduced luminescence of the QD layer [Kai06]. The SRL is directly deposited on the QDs after the GRI. By employing a SRL, an intermediate step in terms of material strain between the highly strained QDs and the lattice constant defining GaAs is created. The additional indium close to the QDs reduces the chemical potential gradient, and consequently leads to the formation of QDs closer to the initial shape of the uncapped QDs which are higher and larger [Son03]. Such QDs embedded in a SRL show a red shifted emission (cf. Figure 4.4] [Max00]. An increase of the indium amount within the SRL leads to an increased QD emission red shift, as shown in Figure 4.5 due to the formation of larger QDs. Although locally reducing the strain of the QDs, the drawback of this technique is a total increase in strain within the complete structure. The larger QDs and increased strain that result makes it increasingly difficult to achieve a strain-free and perfectly flat surface with excellent crystalline quality upon overgrowth of the QDs. This is particularly true for devices where multiple QD layers are required such that the stacking becomes more and more difficult for QDs employing SRL.

\section{V/III ratio}

QD formation is driven by a total energy reduction of the system, leading to material transfer on the surface to the growing QDs. This process is limited by the surface free 


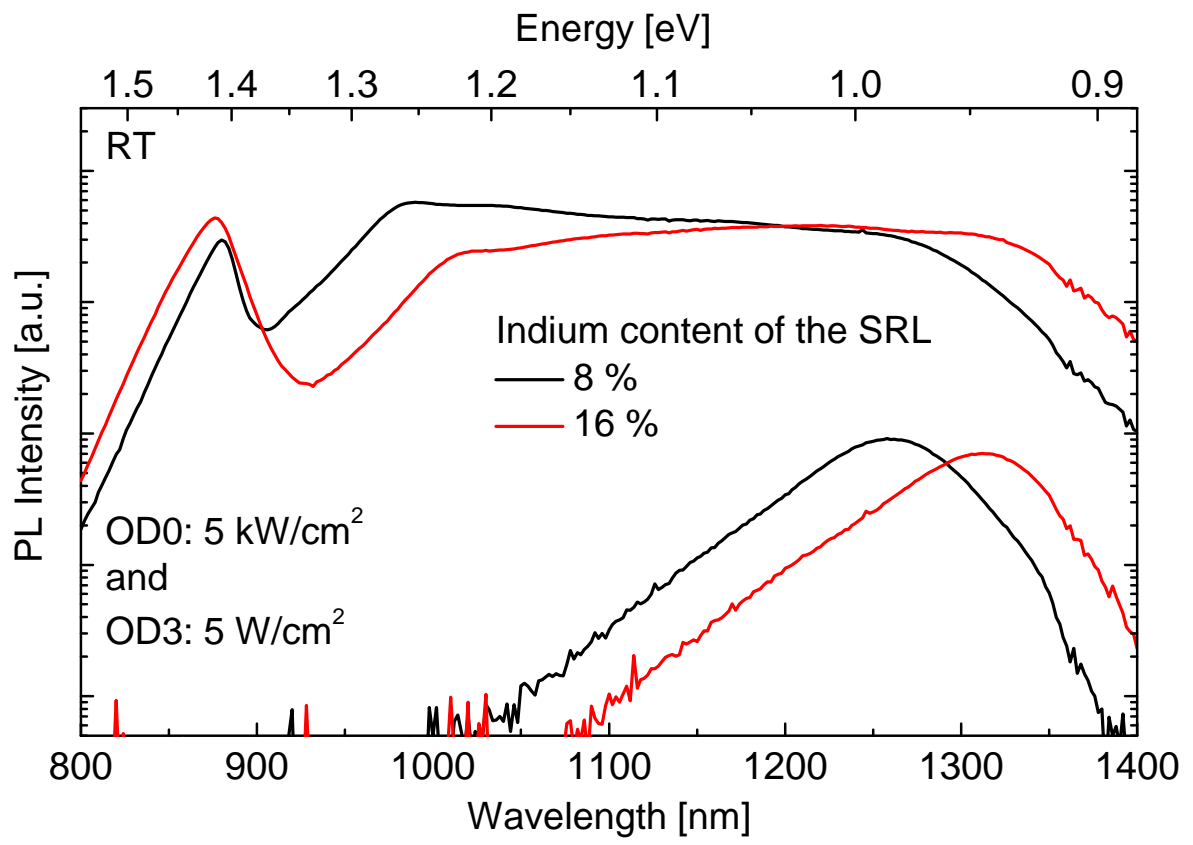

Figure 4.5: An indium content increase from $8 \%$ to $16 \%$ within the SRL causes a significant red shift of $54 \mathrm{~nm}$ to $1312 \mathrm{~nm}$ of the $I n_{0.65} G a_{0.35} A s$ QDs. This shift is linked to a QD peak luminescence decrease of $\approx 10 \%$.

energy of the adatoms, which depends on the arsenic partial pressure within the reactor [Qia88]. Thus the ratio of the group V to the group III precursor material supplied to the MOVPE reactor is a key growth parameter that is termed V/III ratio. While this ratio is a nominal value, the real or surface V/III ratio at the wafer surface can be significantly different. This is caused by material-specific decomposition rates, which are temperature dependent and influenced by catalytic effects in the gas phase and on the wafer surface. Thus the surface V/III ratio is sensitive to the growth temperature. Additionally, nominal values of $\mathrm{AsH}_{3}$ and TBA-based growth are difficult to compare, due to the completely different thermal decomposition properties of these precursors (the same applies to $\mathrm{PH}_{3}$ and TBP). Due to the fact that the surface V/III ratio cannot be easily accessed the nominal V/III ratios for TBAs and TBP are used within this work unless otherwise mentioned.

The V/III ratio has a significant influence on the formation of QDs and their properties up to a suppression of QD luminescence for extremely high V/III ratios [Hei97a]. The reduction of QD luminescence for high V/III ratios during QD deposition and GRI can be explained by the increased formation of large clusters due to the increased surface energies, and thus the higher mobility of indium adatoms [Leo98]. For In GaAs QDs in a GaAs matrix, Passaseo et al. showed that a strong shift in the emission wavelength is possible by changing the V/III ratio for the GaAs capping layer [Pas04]. A similar effect is found for the V/III ratio of the SRL as shown in Figure 4.6. This series shows that an optimum V/III 


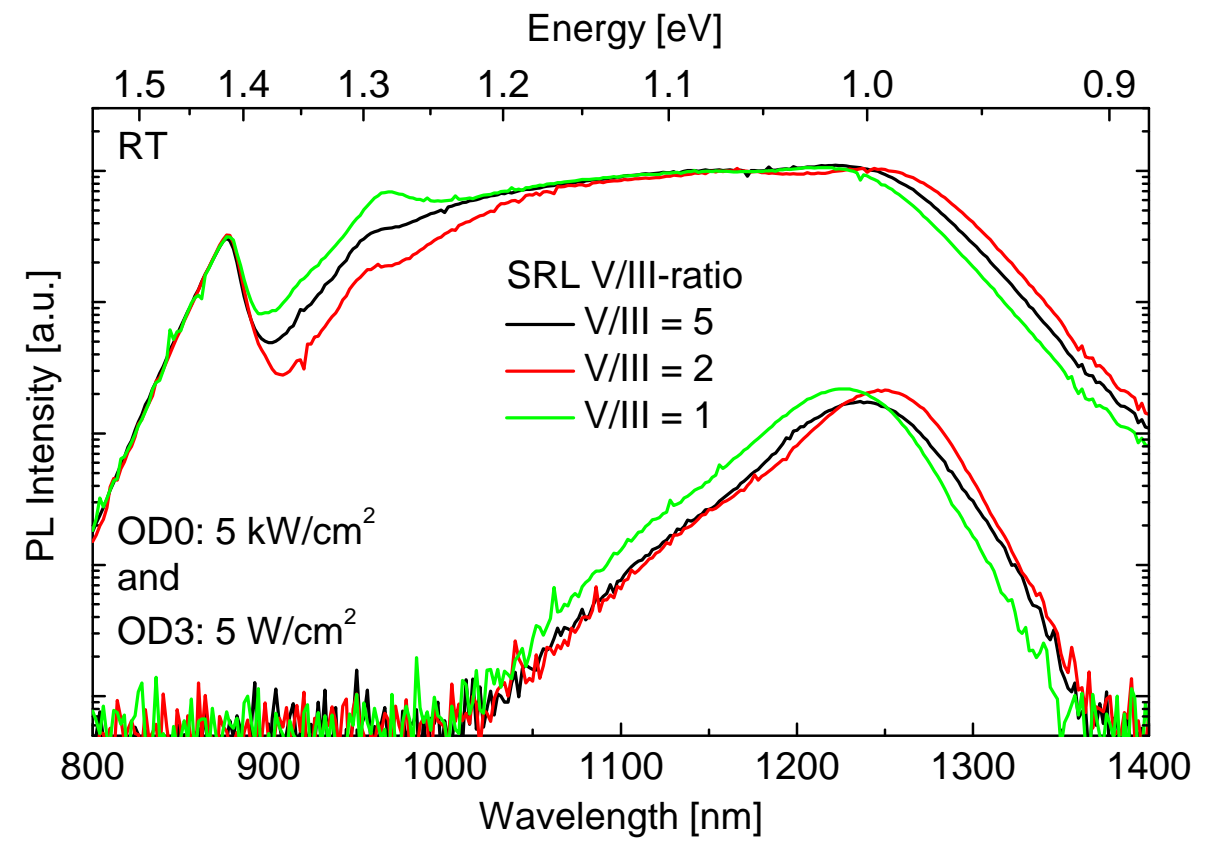

Figure 4.6: PL measurements of samples containing a single InGaAs QD layer covered by $I n_{0.08} G a_{0.92} A s$ SRLs grown at altered V/III ratios. This variation shows an optimum FWHM, red shift and intensity for a SRL V/III ratio of 2.

ratio exists for the QD capping process. An initial reduction from 5 to 2 resulted in a $14 \mathrm{~nm}$ red shift combined with a $21 \%$ intensity increase of the OD3 luminescence. Additionally, the reduction of the full width at half maximum (FWHM) from 82 to $72 \mathrm{~nm}$ shows that the QD ensemble became more homogeneous. A further reduction of the V/III ratio from 2 to 1 resulted in a strong $24 \mathrm{~nm}$ QD emission blue shift, combined with a WL red shift, and increase in WL intensity. This finding is attributed to suppression of the indium transport to the QDs from the SRL during overgrowth, due to the reduced arsenic supply. This led to the formation of a thicker InGaAs WL exhibiting a red shifted emission, and in smaller QDs, which are consequently blue shifted.

\subsubsection{QD stacking optimization}

The maximum density of optically-active QDs limits the optical gain of a single QD layer. Maximum SK-QD densities up to $10^{11} 1 / \mathrm{cm}^{2}$ can be achieved. Stacking of the QD layers with uniform properties is required to further increase the gain within a device. In GaAs QDs are usually embedded into a GaAs matrix which forms the cavity or waveguide. To use this available volume as efficiently as possible, the $G a A s$ spacer within the QD stack needs to be as thin as possible while maintaining excellent crystalline quality, ensuring a perfectly flat and strain-free surface for subsequent QD layers. A PL test structure 


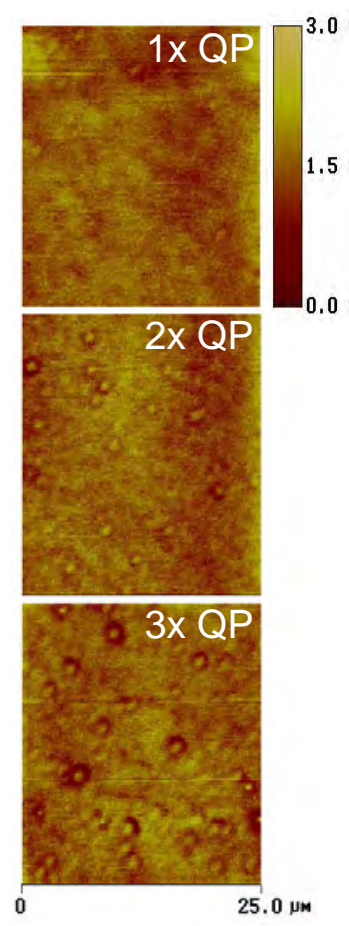

\section{Energy $[\mathrm{eV}]$}

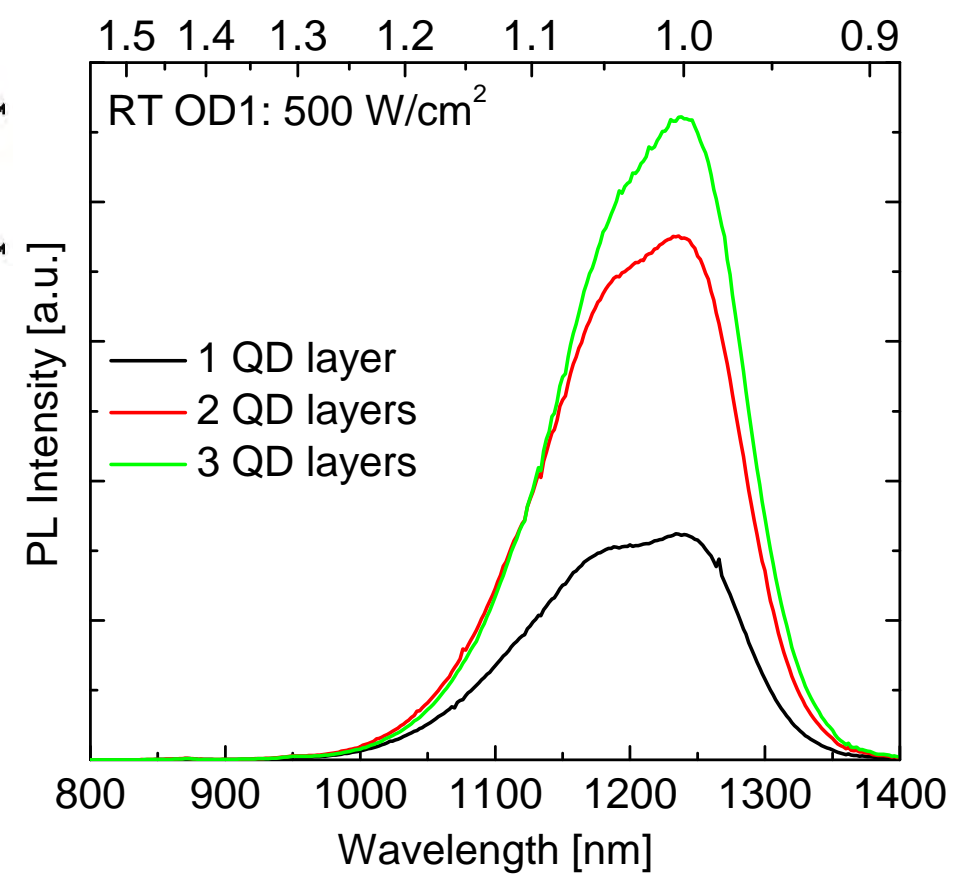

Figure 4.7: PL test samples with 1,2 or $3 I n_{0.65} G a_{0.35} A s$ QD layers with $I n_{0.08} G a_{0.92} A s$ SRL, GaAs spacer thickness is $45 \mathrm{~nm}$ and total matrix thickness is kept constant left: AFM surface images of the complete PL test structures show increasing roughness. right: Corresponding PL measurements at high excitation densities show a sub linear increase for more than 2 QD layers.

design with constant $G a A s$ matrix volume as described in 3.3 was employed to assess the stacking quality of multiple QD layers. Thus a change in the number of embedded identical QD layers does not affect the measured OD3 PL intensity unless additional non-radiative defects are incorporated, or the properties of subsequent QD layers change upon stacking. In contrast, high excitation PL measurements from these PL test samples show the scalability of the QD growth process, and should ideally show a ground state luminescence increase proportional to the number of grown QD layers (cf. Figure 3.3).

\section{Stacking height}

Effective stacking height of QD layers is limited by the total incorporated strain that increases with each additional InGaAs QD layer, and the defect formation upon QD integration into the surrounding GaAs Matrix. A single QD layer with its SRL has a thickness of about $5 \mathrm{~nm}$ with an average indium content of $25 \%$ to $30 \%$, which is well below the critical layer thickness of comparable InGaAs QWs [Eke91, Hsu92]. Due to the inhomogeneous indium distribution within the QD layer, local strain at individual QDs is increased and defects are more easily formed. This incorporated strain and the defect 


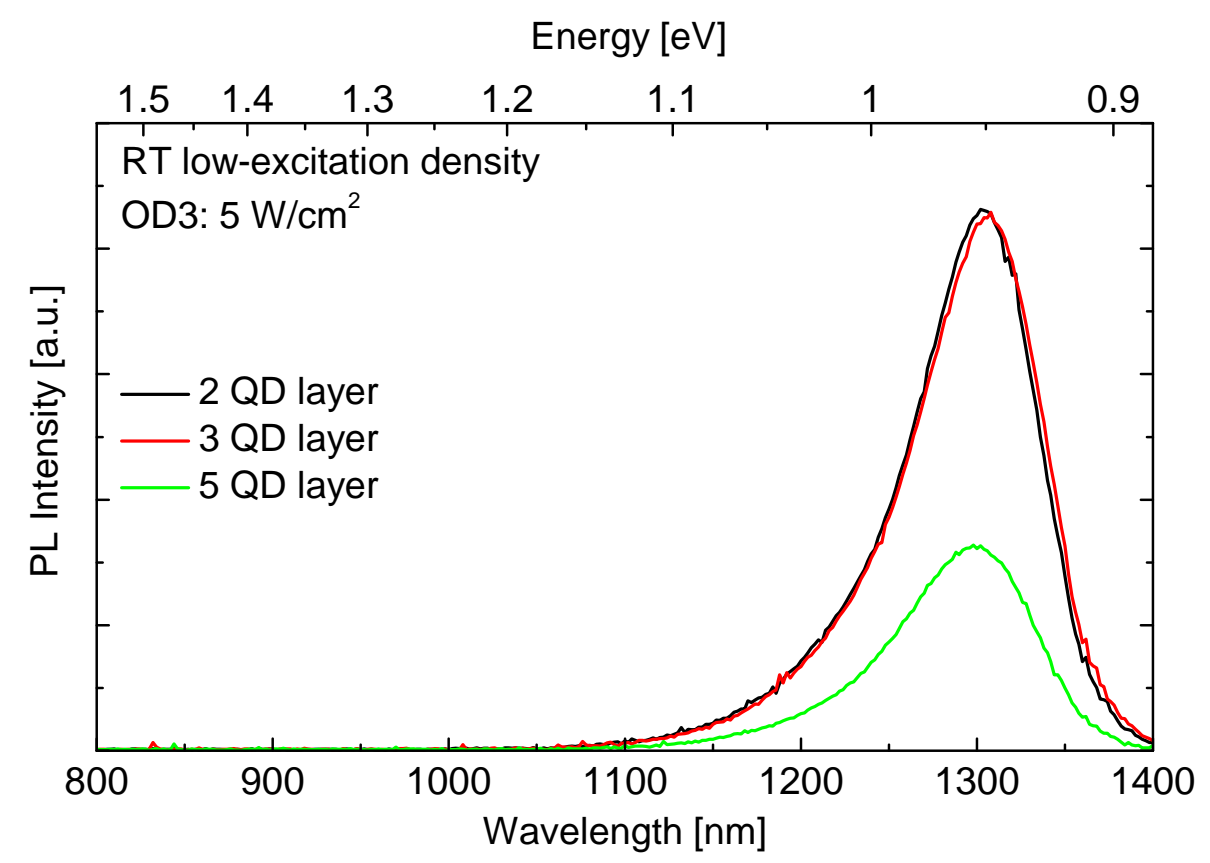

Figure 4.8: Evolution of low excitation density PL with an increasing number of stacked QD layers under non-ideal growth conditions. Total matrix thickness is kept constant.

propagation need to be overcome by effective spacer growth in order to achieve a perfectly flat surface before the deposition of any subsequent QD layer [Sel00]. To reduce the necessary spacer thickness, strain-compensating mechanisms can be introduced, but these can act as additional barriers and may cause problems at hetero-interfaces [Nun07, Suz08]. Also completely defect-free QDs create a strain field influencing the surrounding material which reaches about $25 \mathrm{~nm}$ depending on the QD size and composition [Han04a, Han04b]. Additionally, closely stacked QDs can electronically couple altering optical properties [Yu99]. If the distance to successively grown QD layers is short, the QD formation process of these layers is influenced by the strain field of buried QDs resulting in vertical correlation and altered QD properties in subsequent layers [Xie95, Eis99].

The maximum stacking height of QD layers depends primarily on the QD size and composition, but also on the on the capping process and the spacer growth, which are essential for the integration of the QDs within the crystal. Thus, optimization of the stacking process is a key element for efficient devices. Figure 4.7 shows the scalability of the luminescence at high-excitation density PL by stacking nominal identical QD layers. The third QD layer already showed a strong sub-linear scaling of the PL, which was associated by a significant increase in the surface roughness. This is evidenced by AFM micrographs of the PL test sample surfaces. The topmost QD layer in these samples was already at a distance of about $70 \mathrm{~nm}$ from the surface. Despite this distance, the surface 


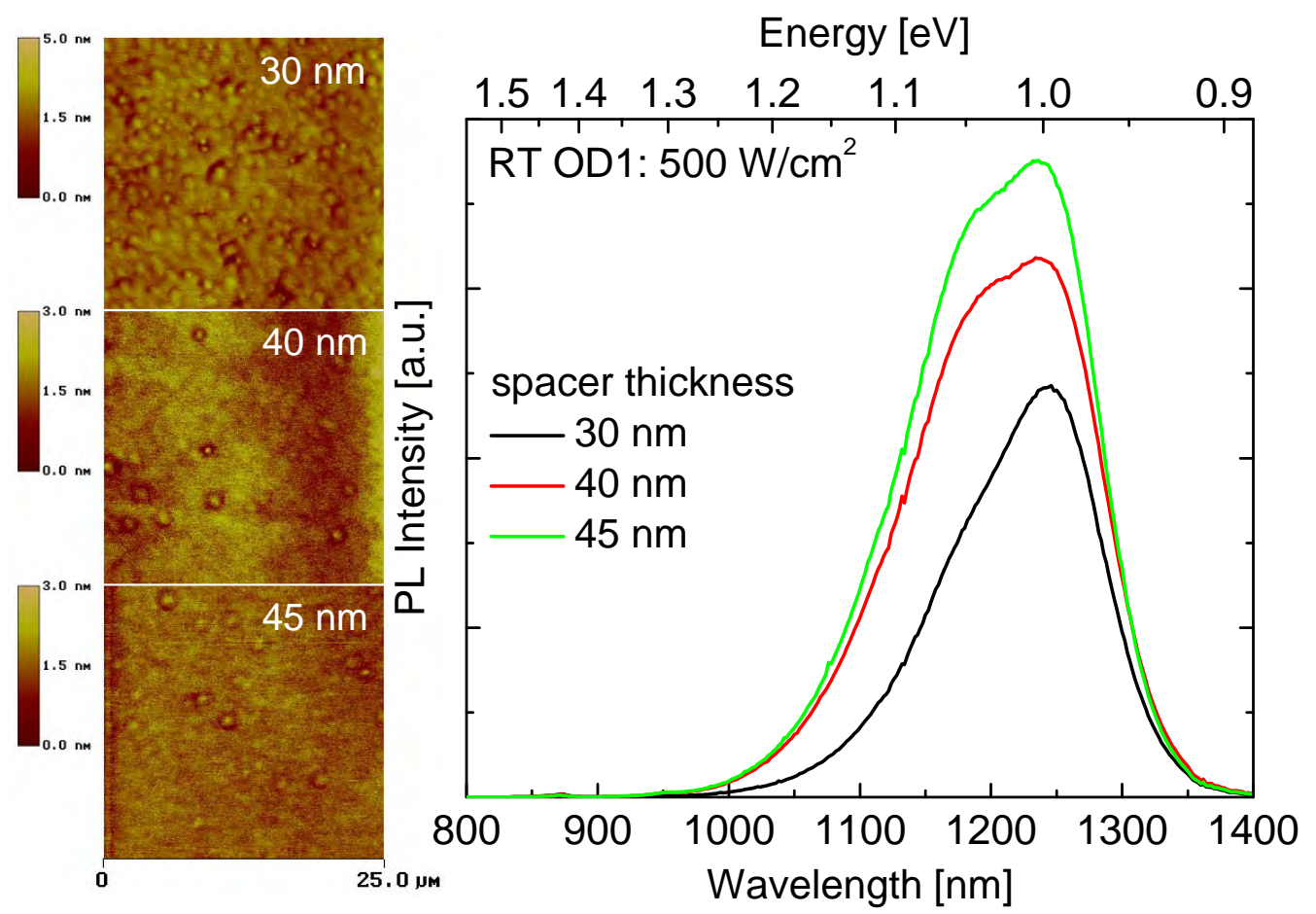

Figure 4.9: PL test samples with varied spacer thickness and two $I n_{0.65} G a_{0.35} A s$ QD layers with $I n_{0.08} G a_{0.92} A s$ SRL left: AFM test structure surface images show a significant reduction in the defect density with increased spacer thickness. right: Corresponding PL measurements show the luminescence increase for varied spacer layers.

defects of the AFM images originate from excessive large QDs or clusters within the active layers, as evidenced by Gradkowski et al. [Gra10]. Another way to assess defect densities upon QD stacking is by low-excitation density PL with photo-generated carrier densities well below QD GS. Figure 4.8 shows such measurements for QD samples emitting at 1.3 $\mu \mathrm{m}$. Samples with 2 or 3 QD layers showed the same luminescence intensity, whereas the optical output of the five-fold QD sample drastically decreased. This decline in ground state PL intensity corresponds to the increased formation of non-radiative defect states in subsequent QD layers caused by a non-ideal stacking growth process. This reinforces that the aim of any spacer growth process is to regain a perfectly smooth surface prior to the growth of a subsequent QD layer.

\section{Spacer growth}

Spacer growth is the most critical part of the QD stacking process. However, the first step of spacer growth is the crucial capping procedure of the previous QD layer, which defines the integration of these QDs into the matrix crystal. Consequently, the resulting surface morphology of this capping layer dictates the challenge for subsequent spacer 
growth in order to regain smooth surface quality. The proximity to the QDs requires the growth of excellent material quality, which is only possible at a sufficiently high growth temperature around $600^{\circ} \mathrm{C}$, whereas $\mathrm{QD}$ growth requires a lower temperature around $500^{\circ} \mathrm{C}$ [Sel00, Liu04]. Thus, spacer growth needs to include variable temperatures including controlled heating and cooling steps. To avoid degradation during such temperature changes, QD layers are capped by a GaAs layer grown at low temperature, and the surface is commonly stabilized by group $\mathrm{V}$ precursor flow to avoid evaporation from the wafer surface. A stack of uniform, uncoupled QD layers necessitates a minimal spacer thickness to eliminate any influence in terms of strain or electronic coupling of the initial QD layer on subsequent layers. This thickness depends on the type of QDs and the growth methods. MBE usually achieves a flat, defect-free surface with a thinner spacer than MOVPE and large, highly strained QDs require a thicker spacer than small QDs. Thin spacers lead to correlated or anti-correlated stacking behavior, which results in increased QD size for subsequent QD layers and vertical coupling effecting the polarization of the QD emission [Yu99, Wan04]. A $45 \mathrm{~nm}$ spacer proved to be the minimum for the investigated QDs for applications close to $1.3 \mu \mathrm{m}$. Figure 4.9 demonstrates the influence of the spacer thickness on the PL intensity and the surface quality of the test samples. An increase beyond $45 \mathrm{~nm}$ for spacers did not result in further improvements in PL intensity for the samples. Annealing steps at the spacer growth temperature can help to further improve the surface morphology [Yan06]. To dissolve overcritical clusters or large QDs, an indium flushing without arsenic stabilization and only partial capping of the QDs can lead to improved stacking behavior [Nun05]. Additionally, strain compensating GaP or GaNAs layers can partially suppress the localized strain field of the QDs and reduce overall strain of the stacked system to achieve a thinner spacer layer [Nun07, Suz08]. The downside of such methods is the integration of additional barriers and interfaces with strain compensating layers, or the non-maximized lateral QD density by the indium flushing method, due to the unused space previously occupied by large dots and clusters.

\section{Individual QD layer optimization}

The aim for QD stack optimization should be to have the same maximized QD quality in terms of density and homogeneity in every single QD layer. A new method was developed in this work to achieve this goal, which is the use of individual GRI times for the initial layer and subsequent layers. This technique takes into account the influence of buried QD layers and compensates for its influence in the following layer by adapting the GRI. Using individual GRI times is particularly effective to restrain the effects of larger QDs such as increased cluster formation in subsequent layers in combination with moderate spacer dimensions. Figure 4.10 shows three-fold QD stack samples with and without individual QD GRI. The results demonstrate a $25 \%$ increase in luminescence and a $75 \%$ reduction 


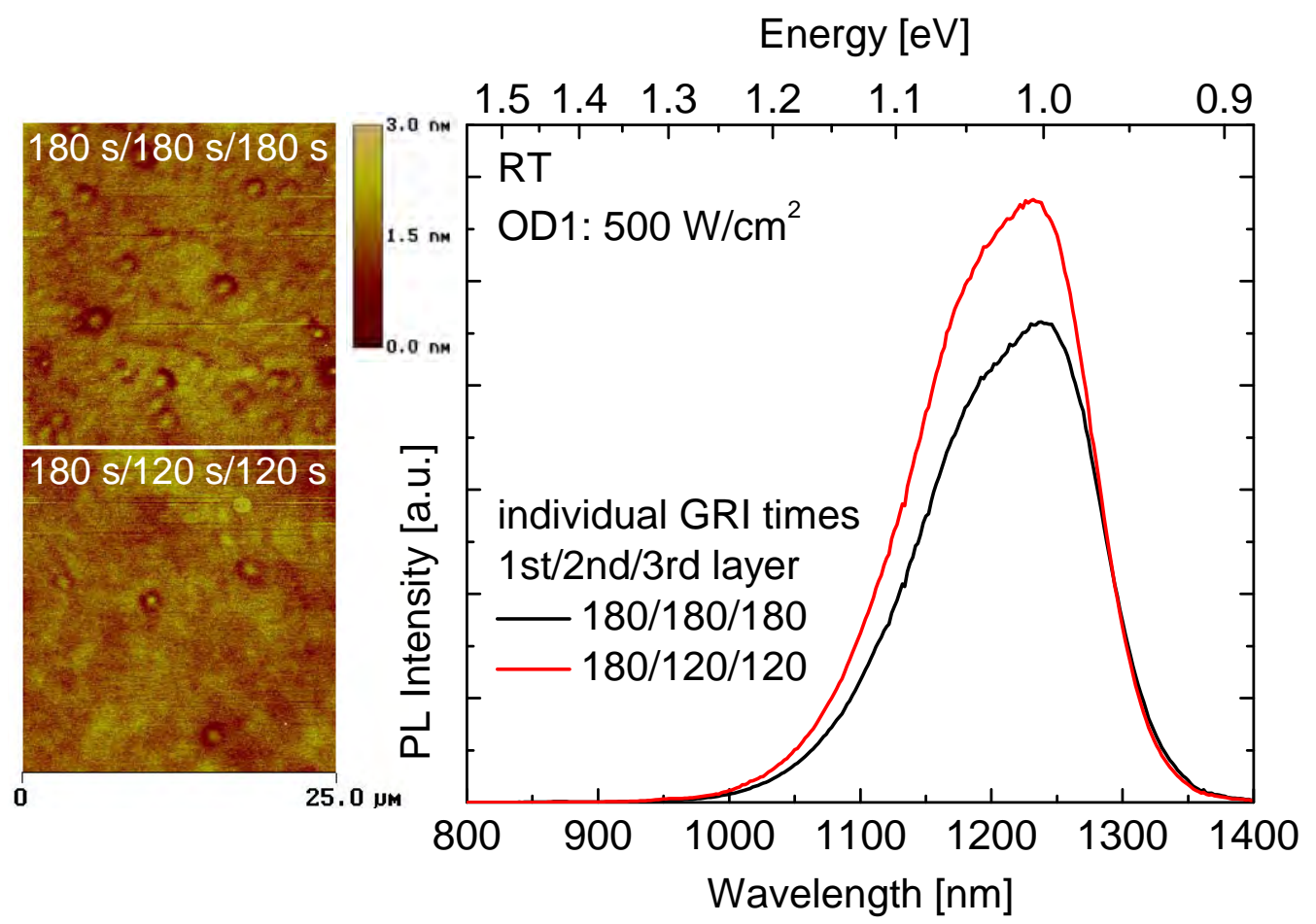

Figure 4.10: Comparison of three-fold $I n_{0.65} G a_{0.35} A s$ QD, $I n_{0.08} G a_{0.92} A s$ SRL PL test samples with constant GRI and individual GRI times and $45 \mathrm{~nm} \mathrm{GaAs}$ spacer. left: AFM surface images of the individual GRI sample show a reduction in defect density by $75 \%$ as compared to the sample with constant GRI times. right: Corresponding PL measurements illustrate a simultaneous increase in luminescence of $25 \%$ for individual GRI times.

in the surface defect density. This QD stacking optimization method was first presented at the annual III/V semiconductors epitaxy workshop 2005 [Ger05]. Ultralow threshold laser devices employing this technique have been fabricated and published by Strittmatter and Germann et al. [Str06, Ger07].

\section{V/III ratio}

As mentioned in 4.1.1 the V/III ratio is an important parameter for QD growth. However for the epitaxy of bulk layers this growth parameter is often underestimated. Usually a large $\mathrm{V} / \mathrm{III}$ is chosen to grow with an arsenic rich $\mathrm{c}(4 \times 4)$-GaAs surface reconstruction, resulting in a huge surplus of arsenic. Typical V/III values employing TMGa, TMAl, and TBAs precursors for GaAs growth and $A l A s$ growth are 15 and 30, respectively. As shown in previous work, the driving diffusion parameter for group III atoms in the AlGaAs system is the concentration of triply charged group III vacancies $\left(V_{I I I}\right)$ [Dep88]. In contrast to a monoatomic crystal such as silicon, the concentration of vacancies and other point defects is not a single value but varies within the narrow compositional range of the GaAs crystal 


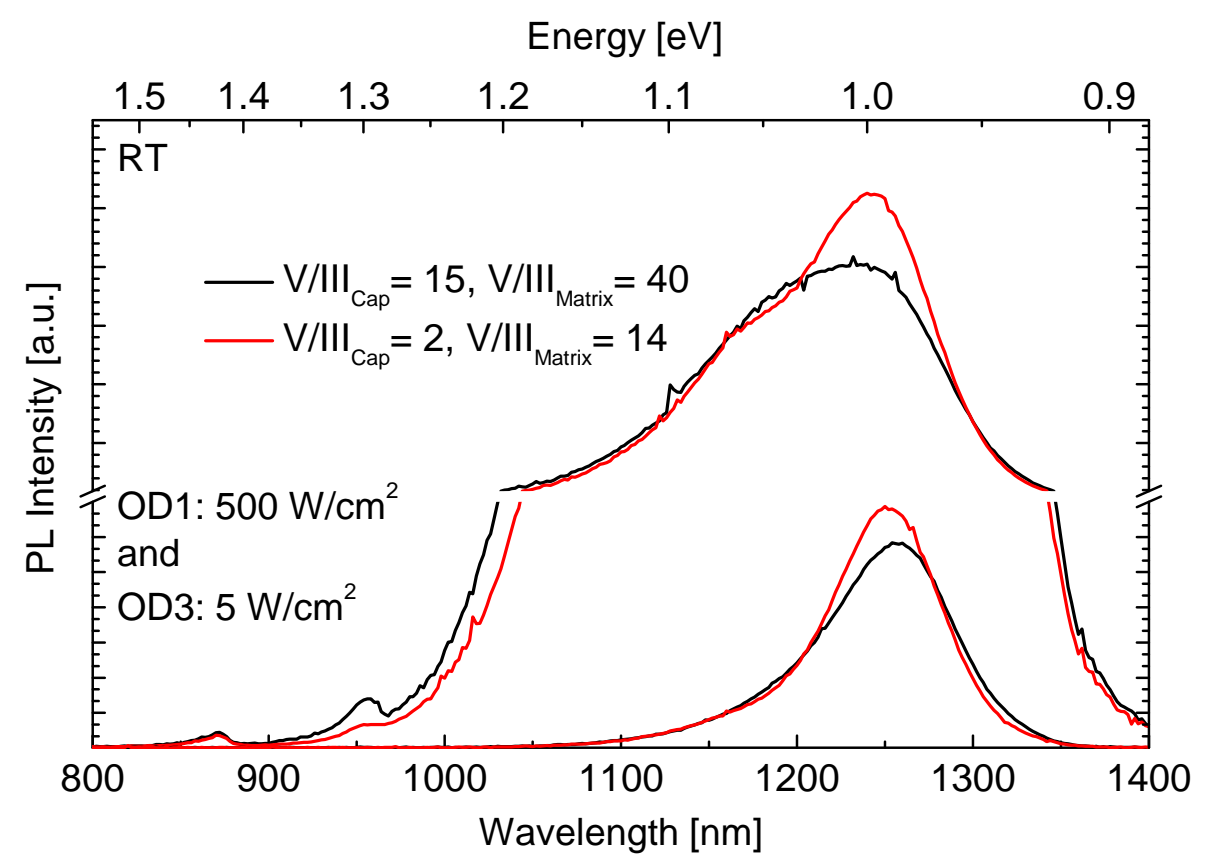

Figure 4.11: Variation of the GaAs matrix V/III ratio grown on top of every QD layer in a three-fold $I n_{0.65} G a_{0.35} A s$ QD, $I n_{0.08} G a_{0.92} A s$ SRL PL test sample. The GaAs spacer matrix is grown in two steps: capping layer at $\mathrm{QD}$ growth temperature $\left(520^{\circ} \mathrm{C}\right)$ and matrix layer at $615^{\circ} \mathrm{C}$. Reduction of the V/III ratio results in an improved luminescence of the stacked sample.

[Tan91]. This results in a thermal equilibrium of the point defect concentration that is dependent upon the material's vapor phase pressures. In an epitaxial reactor, pressure ratios are given by the V/III ratio, which can be chosen by the operator. Thus V/III ratios are a handle to influence point defect concentration and $V_{I I I}$-density within the crystal. Additionally, high V/III ratios lead to arsenic-rich surface reconstructions. In this way the surface represents a thin layer with the highest possible $V_{I I I}$-density. This becomes especially important for the growth of strained material where vacancy-driven diffusion adds up to the strain-driven diffusion. QDs with a locally increased strain within the crystal are especially vulnerable to this effect, resulting in unintentional indium diffusion out of the QDs into the surrounding GaAs matrix. This effect was used by Passaseo et al. [Pas04] to tune the emission wavelength of In GaAs QDs by changing the V/III ratio of the $G a A s$ capping layer. In Figure 4.11 the V/III ratio of the complete $G a A s$ matrix grown on top of every QD layer was changed in a three-fold sample, resulting in an increased peak luminescence and reduced FWHM from $86 \mathrm{~nm}$ to $74 \mathrm{~nm}$. This, in turn, resulted in a more efficient stacking behavior. These results are attributed to the reduced V/III ratio, which allows for a better integration of the QDs within the matrix crystal, and reduces the typical QD ensemble broadening. The question that arises is, at which distance to the QDs does this effect end? Figure 4.12 shows only the influence of the GaAs matrix V/III 


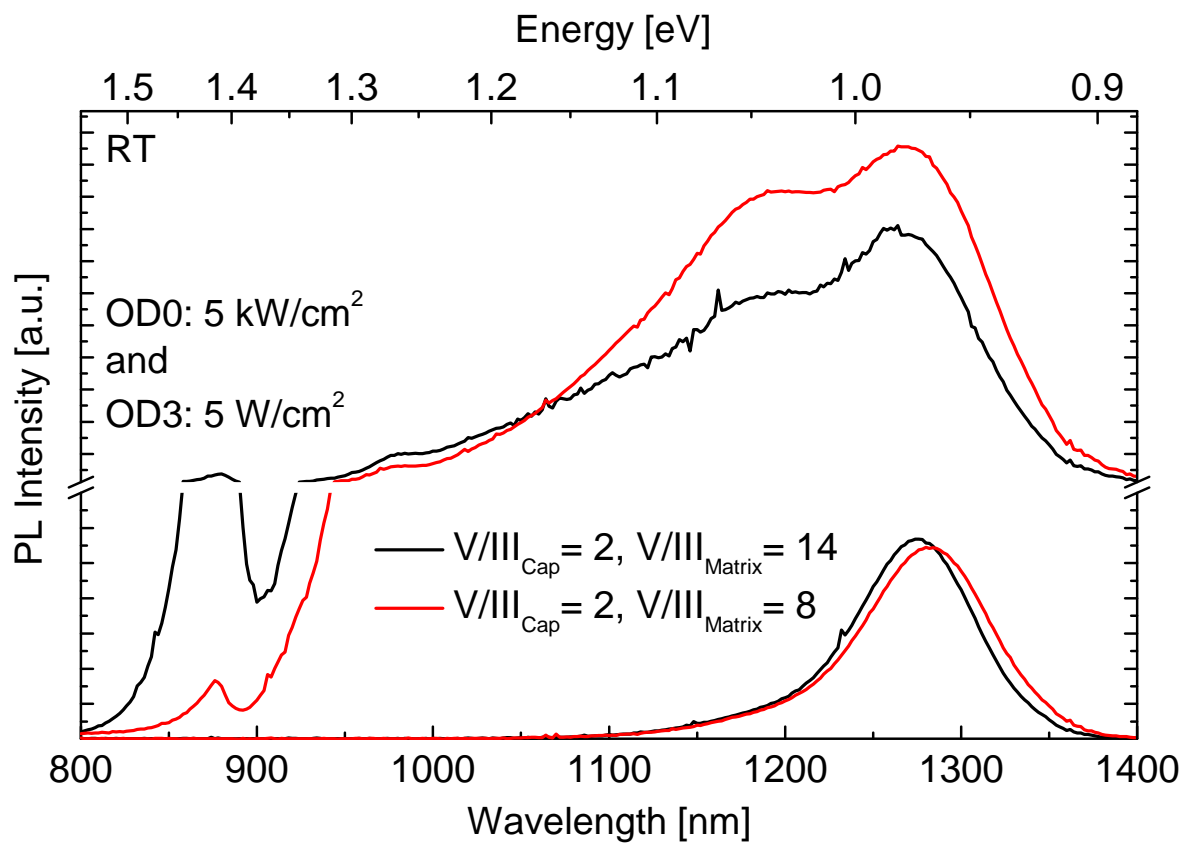

Figure 4.12: Comparison of three-fold $I n_{0.72} G a_{0.28} A s \mathrm{QD}, I n_{0.12} G a_{0.88} A s$ SRL PL test samples. Results of this reduction of the $\mathrm{GaAs}$ spacer V/III ratio are a $30 \%$ OD0 peak luminescence increase and an $8 \mathrm{~nm}$ red shift.

ratio used for spacer growth in a three-fold sample structure. This data demonstrates the important role of the V/III ratio during overgrowth of QD structures. While the FWHM of both samples differs only slightly, an $8 \mathrm{~nm}$ red shift is achieved which is attributed to the reduced indium inter diffusion, due to a reduced $V_{I I I}$-concentration generated during growth. Additionally, the reduction of the V/III ratio results in a significant improvement of the QD stacking quality, as evidenced by the $30 \%$ increase in peak luminescence for the high excitation density. Note: the employed QDs and SRL already contain an increased indium content with respect to the samples in Figure 4.11 . 


\subsection{Doping and material quality}

This section gives an overview of typical material qualities used for growth of laser devices within this work. This means that no record values are reported, which are only achievable under special conditions for singular samples and do not reflect material characteristics within complex structures for applications. Instead, realistic material properties are depicted which are sufficient for the outstanding laser performance of devices demonstrated in the following Chapters 5,6 and 7

Initial structures grown with the employed Aixtron 200/4 system were restricted to alternative precursors. TBAs as arsenic precursor and TBP as phosphorus precursor are employed for the group $\mathrm{V}$ elements, and $\mathrm{CBr}_{4}$ and DETe are used as $\mathrm{p}$ and $\mathrm{n}$ dopant sources, respectively. In contrast to hydride sources, metalorganic sources show a significantly lower vapor pressure. Thus, they are less hazardous in terms of fires or explosions. Nevertheless, $\mathrm{AsH}_{3}$ and $\mathrm{SiH}_{4}$ sources are well established in industry and can be advantageous in terms of cost and material quality. Consequently, a diluted $\mathrm{SiH}_{4}$ source was added in March 2006 to the MOVPE system. Later, in January 2008, the system was extended by an $\mathrm{AsH}_{3}$ line as an alternative to TBAs in order to broaden the experimental possibilities.

Doped and undoped samples were investigated by the van der Pauw method as described in the Appendix A.2.2 to assess free charge carrier concentrations and mobility. Results of the most relevant material compositions for the fabricated lasers are shown in Tables 4.1$G a A s, 4.2-A l_{0.8} G a_{0.2} A s$ and 4.4- lattice matched $I_{0.48} G a_{0.52} P$.

\subsubsection{GaAs}

As a binary material, growth of GaAs is mostly unproblematic for a wide range of possible growth parameters (cf. Table 4.1). Growth of GaAs with TBAs already demonstrates excellent intrinsic values down to background doping levels of $n=2.8 \cdot 10^{14} \mathrm{~cm}^{-3}$ at $615^{\circ} \mathrm{C}$ with $\mathrm{V} / \mathrm{III}=9$, which compare well to literature [Che87a, Lum87]. This value suffers from the poor charge carrier mobility achieved by the sample, indicating a compensation mechanism at low temperatures. An increased growth temperature and V/III ratio leads to an increase of the free charge carriers and RT mobility. In contrast to TBAs, $\mathrm{AsH}_{3}$ based growth shows comparable intrinsic doping levels combined with significantly improved charge carrier mobility for high V/III ratios. Good crystalline quality for both arsenic precursors is achievable up to growth rates of 2 to $3 \mu \mathrm{m} / \mathrm{h}$. As $A s H_{3}$ needs high temperatures for thermal decomposition, TBAs remains preferential for ultra-low temperature growth, whereas above $600^{\circ} \mathrm{C} \mathrm{AsH}_{3}$ can improve material quality and is simultaneously more cost-effective. Thus, for all samples of this work low temperature growth around $500^{\circ} \mathrm{C}$, e.g. for QD active layers, is always carried out with TBAs. Process 


\begin{tabular}{|c|c|c|c|c|c|c|c|c|}
\hline 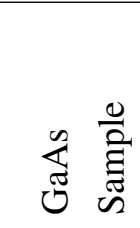 & $\underset{F}{\overrightarrow{3}}$ & $\begin{array}{l}\overrightarrow{\tilde{\Xi}} \\
\text { ڤ̆ }\end{array}$ & 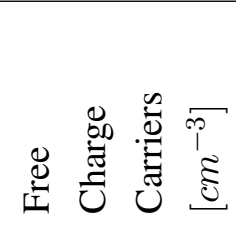 & 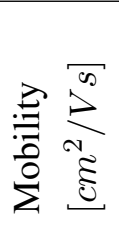 & 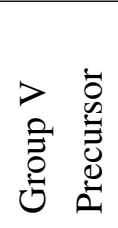 & 总 & 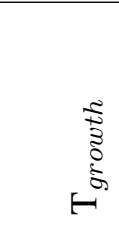 & 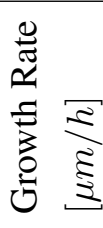 \\
\hline \multirow{2}{*}{ Np2372 } & RT & int. & $n=2.8 \cdot 10^{14}$ & 910 & TBAs & 9 & $615^{\circ} \mathrm{C}$ & 0.6 \\
\hline & $77 \mathrm{~K}$ & int. & $n=3.1 \cdot 10^{12}$ & 4430 & TBAs & 9 & $615^{\circ} \mathrm{C}$ & 0.6 \\
\hline \multirow{2}{*}{ Np3973 } & RT & int. & $n=2.8 \cdot 10^{15}$ & 2420 & TBAs & 14 & $700^{\circ} \mathrm{C}$ & 0.7 \\
\hline & $77 \mathrm{~K}$ & int. & $n=2.4 \cdot 10^{15}$ & 1080 & TBAs & 14 & $700^{\circ} \mathrm{C}$ & 0.7 \\
\hline \multirow{2}{*}{ Np3678 } & RT & int. & $n=1.4 \cdot 10^{15}$ & 2940 & $\mathrm{AsH}_{3}$ & 86 & $700^{\circ} \mathrm{C}$ & 2.2 \\
\hline & $77 \mathrm{~K}$ & int. & $n=6.3 \cdot 10^{14}$ & 6950 & $\mathrm{AsH}_{3}$ & 86 & $700^{\circ} \mathrm{C}$ & 2.2 \\
\hline \multirow{2}{*}{ Np4001 } & RT & int. & $n=9.5 \cdot 10^{14}$ & 3240 & $\mathrm{AsH}_{3}$ & 154 & $700^{\circ} \mathrm{C}$ & 2.0 \\
\hline & $77 \mathrm{~K}$ & int. & $n=2.9 \cdot 10^{14}$ & 45100 & $\mathrm{AsH}_{3}$ & 154 & $700^{\circ} \mathrm{C}$ & 2.0 \\
\hline Np2017 & RT & DETe & $n=5.5 \cdot 10^{18}$ & 1820 & TBAs & 15 & $700^{\circ} \mathrm{C}$ & 2.0 \\
\hline Np3692 & RT & $\mathrm{SiH}_{4}$ & $n=4.9 \cdot 10^{18}$ & 1480 & $\mathrm{AsH}_{3}$ & 206 & $700^{\circ} \mathrm{C}$ & 1.1 \\
\hline Np2018 & RT & $\mathrm{CBr}_{4}$ & $p=1.0 \cdot 10^{19}$ & 84 & TBAs & 5 & $615^{\circ} \mathrm{C}$ & 1.6 \\
\hline Np3688 & RT & DMZn & $p=4.7 \cdot 10^{18}$ & 88 & $\mathrm{AsH}_{3}$ & 175 & $700^{\circ} \mathrm{C}$ & 1.3 \\
\hline Np3705 & $\mathrm{RT}$ & $\mathrm{DMZn}$ & $p=1.7 \cdot 10^{19}$ & 60 & $\mathrm{AsH}_{3}$ & 175 & $700^{\circ} \mathrm{C}$ & 1.3 \\
\hline
\end{tabular}

Table 4.1: Hall-measurements of selected GaAs samples represent common properties of materials employed within the realized laser devices. This Table presents values achieved with corresponding growth parameters. Growth is not especially optimized for record-low intrinsic values. Doped samples show a smooth surface morphology and are suitable for device fabrication.

parts at growth temperatures of $600^{\circ} \mathrm{C}$ and above were switched to $\mathrm{AsH}_{3}$ as soon as it was available.

Initially, DETe was used as a donor source for highly doped n-material. The use of this alternative precursor results in unproblematic doping up to $n \approx 1 \cdot 10^{19} \mathrm{~cm}^{-3}$, however tellurium shows a memory effect which can cause unintentional doping of subsequent layers and samples [Hou86]. In the case of the employed Aixtron 200/4 system, reactorconditioning runs became necessary after the growth of heavily tellurium-doped samples. As soon as available, tellurium was replaced by $\mathrm{SiH}_{4}$, which allows for similar doping results up to $n \approx 5 \cdot 10^{18} \mathrm{~cm}^{-3}$, without the unintended memory effect. Beyond this level, a further increase of the supplied $\mathrm{SiH}_{4}$ causes a self-compensating effect, preventing higher doping levels due to silicon's group IV character. This allows for incorporation on both group III and group V sites.

$\mathrm{CBr}_{4}$ as a carbon acceptor source shows very good results up to $p \approx 1 \cdot 10^{19} \mathrm{~cm}^{-3}$ and exhibits a very low diffusivity [Mal88]. In MOCVD $\mathrm{CBr}_{4}$ becomes critical at high concentration levels due to its etching effects. In consequence material quality is affected, 
resulting in the formation of defects already visible at the wafer surface. DMZn achieved similar doping levels but needs to be regarded critically due to its high diffusion coefficient in GaAs [Gös81, v083]. As described in Section 4.1.2, group V partial pressure, and thus the V/III ratio, influences $V_{I I I}$ formation. Due to the vacancy-driven $Z n$-diffusion mechanism, diffusion coefficients depend on group $\mathrm{V}$ partial pressure during growth, heating, and growth interruptions. Additionally, the final $Z n$-diffusion length within the crystal relies on material temperature and $Z n$-concentrations.

\subsubsection{AIGaAs}

The lattice constant of $A l A s$ is only slightly larger than that of $G a A s(\approx 0.1 \%)$ [Ada85]. Thus, all ternary $A l_{x} G a_{1-x} A s$ compositions can easily be grown on $G a A s$ substrates up to a layer thickness of more than one micron. For very thick layers, carbon incorporation can be used for strain compensation [Maz96]. Carbon incorporation also takes place intrinsically as high levels of carbon acceptors often originate from the organic part of metal-organic precursor molecules and are incorporated during growth. The level of this intrinsic p-doping of nominally undoped layers can be influenced by growth parameters as the growth temperature and the V/III ratio [Got04]. Additionally, aluminum has a strong intrinsic affinity to oxygen, which makes it very sensitive for precursor impurities. Thus, the use of $\mathrm{AsH}_{3}$ with non-optimal quality can lead to increased oxygen incorporation, depending on the V/III ratio [Kak96], whereas TBAs-based growth shows a V/III ratio that is almost independent of oxygen concentration [Leu98]. Different growth conditions for $\mathrm{Al}_{0.8} \mathrm{Ga}_{0.2} \mathrm{As}$ layers employing TBAs and $\mathrm{AsH}_{3}$ were investigated by Hall measurements and are summarized in Table 4.2

TBAs-based $A l_{0.8} G a_{0.2} A s$ showed a significantly lower charge carrier concentration for a growth temperature of $615^{\circ} \mathrm{C}$ as compared to $700^{\circ} \mathrm{C}$. This is in good agreement with the reported carbon incorporation dependence during growth by Leu et al. [Leu98]. A variation of the V/III ratio showed only minor influence on the intrinsic doping properties. The best samples are grown with a fast growth rate of $2.5 \mu \mathrm{m} / \mathrm{h}$, achieving a low intrinsic charge carrier level of $p=2.1 \cdot 10^{16} \mathrm{~cm}^{-3}$, and high charge carrier mobility of $110 \mathrm{~cm}^{2} / \mathrm{Vs}$.

$A l_{0.8} G_{0.2} A s$ growth based on $\mathrm{AsH}_{3}$ produces similar results but allows for a wider range of growth parameters regarding growth rate and temperature. The best results are achieved at $700^{\circ} \mathrm{C}$, which can most likely be attributed to the more efficient thermal $\mathrm{AsH}_{3}$ decomposition. Thus, the effective V/III ratio on the wafer surface is increased, which leads to an improved metal-organic radical removal by the larger amount of available atomic hydrogen. Consequently, reduced growth temperatures require even higher nominal V/III ratios to realize optimum material quality [Cho98]. This is supported by a sample with a five-fold increased V/III ratio compared to sample Np4333 (low growth rate at $615^{\circ} \mathrm{C}$ ), which could not be measured with the available Hall setup due to its increased, 


\begin{tabular}{|c|c|c|c|c|c|c|c|c|}
\hline 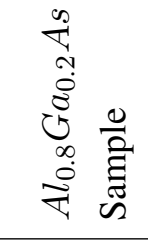 & $\overrightarrow{\widetilde{\Xi}}$ & 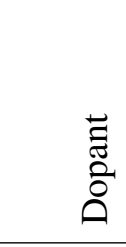 & 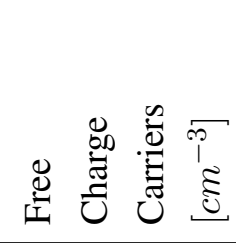 & 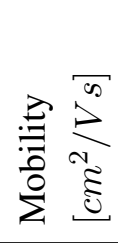 & 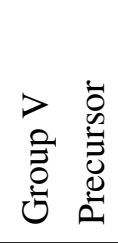 & 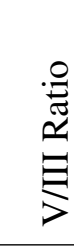 & 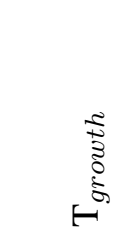 & 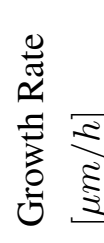 \\
\hline \multirow{2}{*}{ Np2026 } & $\mathrm{RT}$ & int. & $p=2.2 \cdot 10^{17}$ & 37 & TBAs & 30 & $615^{\circ} \mathrm{C}$ & 1.4 \\
\hline & $77 \mathrm{~K}$ & int. & $p=9.4 \cdot 10^{15}$ & 1010 & TBAs & 30 & $615^{\circ} \mathrm{C}$ & 1.4 \\
\hline \multirow{2}{*}{ Np2564 } & $\mathrm{RT}$ & int. & $p=2.1 \cdot 10^{16}$ & 110 & TBAs & 30 & $615^{\circ} \mathrm{C}$ & 2.5 \\
\hline & $77 \mathrm{~K}$ & int. & $p=1.1 \cdot 10^{16}$ & 900 & TBAs & 30 & $615^{\circ} \mathrm{C}$ & 2.5 \\
\hline \multirow{2}{*}{ Np2619 } & $\mathrm{RT}$ & int. & $p=1.1 \cdot 10^{18}$ & 36 & TBAs & 29 & $700^{\circ} \mathrm{C}$ & 2.8 \\
\hline & $77 \mathrm{~K}$ & int. & $p=4.3 \cdot 10^{16}$ & 490 & TBAs & 29 & $700^{\circ} \mathrm{C}$ & 2.8 \\
\hline \multirow{2}{*}{ Np3279 } & RT & int. & $p=3.8 \cdot 10^{16}$ & 150 & $\mathrm{AsH}_{3}$ & 235 & $700^{\circ} \mathrm{C}$ & 2.5 \\
\hline & $77 \mathrm{~K}$ & int. & $p=5.6 \cdot 10^{15}$ & 310 & $\mathrm{AsH}_{3}$ & 235 & $700^{\circ} \mathrm{C}$ & 2.5 \\
\hline \multirow{2}{*}{ Np4329 } & $\mathrm{RT}$ & int. & $p=1.6 \cdot 10^{17}$ & 69 & $\mathrm{AsH}_{3}$ & 235 & $615^{\circ} \mathrm{C}$ & 2.5 \\
\hline & $77 \mathrm{~K}$ & int. & $p=1 \cdot 10^{16}$ & 1000 & $\mathrm{AsH}_{3}$ & 235 & $615^{\circ} \mathrm{C}$ & 2.5 \\
\hline \multirow{2}{*}{ Np4333 } & RT & int. & $p=4.7 \cdot 10^{16}$ & 110 & $\mathrm{AsH}_{3}$ & 250 & $615^{\circ} \mathrm{C}$ & 0.5 \\
\hline & $77 \mathrm{~K}$ & int. & $p=2.8 \cdot 10^{16}$ & 150 & $\mathrm{AsH}_{3}$ & 250 & $615^{\circ} \mathrm{C}$ & 0.5 \\
\hline Np2469 & RT & DETe & $n=8.0 \cdot 10^{18}$ & 100 & TBAs & 28 & $615^{\circ} \mathrm{C}$ & 1.7 \\
\hline Np2665 & RT & $\mathrm{SiH}_{4}$ & $n=3.2 \cdot 10^{17}$ & 1390 & TBAs & 29 & $700^{\circ} \mathrm{C}$ & 2.8 \\
\hline Np2344 & RT & $\mathrm{CBr}_{4}$ & $p=3.2 \cdot 10^{18}$ & 41 & TBAs & 30 & $615^{\circ} \mathrm{C}$ & 2.4 \\
\hline Np3713 & RT & DMZn & $p=1.1 \cdot 10^{18}$ & 38 & $\mathrm{AsH}_{3}$ & 134 & $700^{\circ} \mathrm{C}$ & 1.6 \\
\hline
\end{tabular}

Table 4.2: Hall-measurements of selected $A l_{0.8} G a_{0.2} A s$ samples with the exception of Np3713 which contains $90 \%$ aluminum. The table depicts common material properties for doped and undoped samples with corresponding growth parameters. Doped samples show a smooth surface morphology and are suitable for device fabrication.

very high resistivity of $10 \mathrm{M} \Omega$, that indicates a further reduction in free charge carriers. As aluminum is highly sensitive to any impurities, thus a very important factor for material properties is the quality of the employed precursor sources. The TMAl quality proved to be especially critical, as usage of material from a different production batch resulted in significantly increased background doping levels of up to $p=1 \cdot 10^{18} \mathrm{~cm}^{-3}$ without any changes in growth parameters.

To grow n-doped $A l_{x} G a_{1-x} A s$ material, the intrinsically available holes need to be compensated for by a larger amount of free electron charge carriers. Doping with tellurium allows for very high doping levels up to $n \approx 1 \cdot 10^{19} \mathrm{~cm}^{-3}$, but there is always the risk of influencing subsequent layers and samples by its memory effect [Hou86, Sun91]. Undoped conditioning runs capping all remaining tellurium-containing material within the reactor are necessary to overcome this effect. With the alternative dopant source, $\mathrm{SiH}_{4}$ maximum 


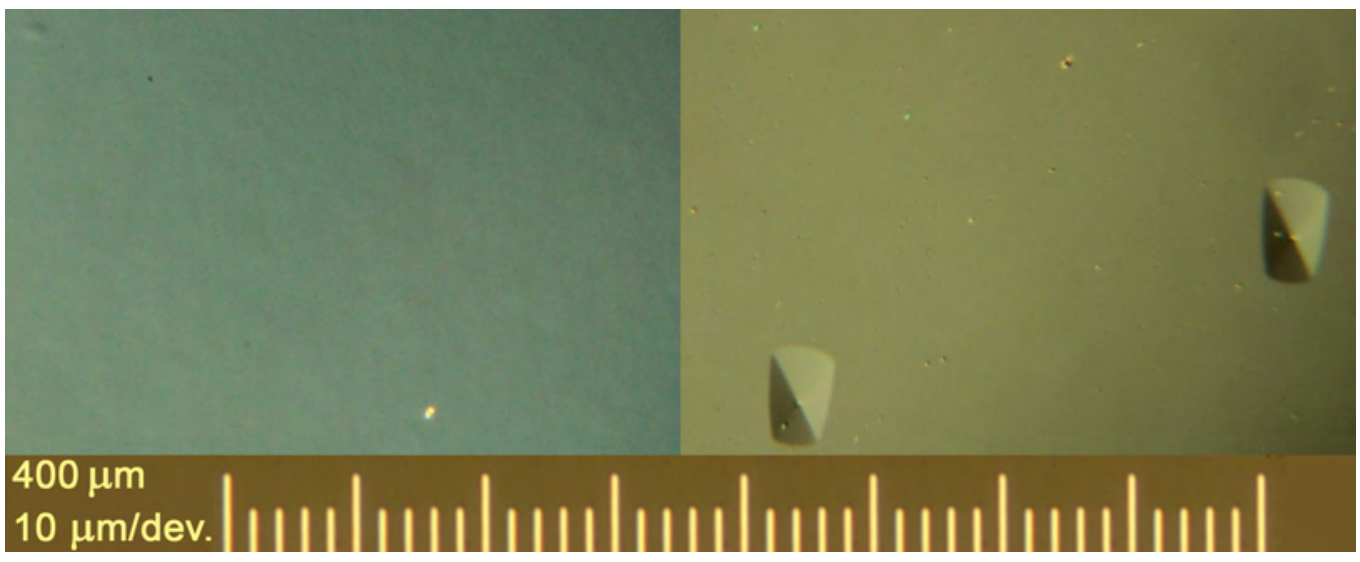

Figure 4.13: Nomarski surface micrographs of undoped $A l G a A s$ samples grown with identical parameters except for the group $\mathrm{V}$ precursors $\left(615^{\circ} \mathrm{C}, 1 \mu \mathrm{m} / \mathrm{h}, 2 \mu \mathrm{m}\right.$ thick $A l_{0.28} G a_{0.72} A s$ layer, 10 $\mathrm{nm}$ GaAs Cap) left: TBAs grown sample with V/III $=21$ right: $A s H_{3}$ grown sample with $\mathrm{V} / \mathrm{III}=$ 375 showing large pyramidal defects.

doping levels are limited to $n<1 \cdot 10^{18} \mathrm{~cm}^{-3}$ for high aluminum contents of $x \geqq 80 \%$, due to its self-compensating behavior of the group IV material silicon. For $x=15 \%$ up to $n=2.6 \cdot 10^{18} \mathrm{~cm}^{-3}$ is achieved.

The intrinsic $A l G a A s$ p-doping behavior, due to its affinity to incorporate carbon, can be used to realize high p-doping levels by choosing appropriate growth parameters, without the need for any additional dopant sources. This intrinsic carbon incorporation mainly depends on the arsenic to group III precursor ratio. A high V/III ratio reduces the intrinsic carbon concentration, and thus the intrinsic doping level, and vice versa. Extremely low $\mathrm{V} / \mathrm{III}$ ratios $<5$ can be used to realize charge carrier concentrations up to $p>1 \cdot 10^{20}$ $\mathrm{cm}^{-3}$, affecting material properties such as the lattice constant [Fuj99, Got04]. Concerning the intrinsic carbon incorporation, TBAs proves to be much more effective than $\mathrm{AsH}_{3}$, as the free hydrogen atoms originating from the $\mathrm{AsH}_{3}$ decomposition are essential for the removal of the carbon containing methyl groups from the metal-organic precursors. This agrees with other reports, which indicate that the intrinsic carbon originates from the TMGa and TMAl molecules and not from TBAs butyl-group [Mas95]. If $\mathrm{p}$ doping is needed and ultra low V/III ratios are not possible due to material quality concerns, $\mathrm{CBr}_{4}$ and DMZn can be used as dopant sources. These precursors yield very high doping levels up to $p=1 \cdot 10^{20} \mathrm{~cm}^{-3}$ [Sun91]. By employing such dopant materials, unwanted side effects such as etching of $\mathrm{CBr}_{4}$ and possible zinc diffusion need to be taken into account. $\mathrm{CBr}_{4}$ can particularly lead to altered $A l G a A s$ compositions due to its differing influence on gallium and aluminum incorporation.

Not all $A l_{x} G a_{1-x} A s$ compositions show the same mirror-like surfaces as the aforementioned $x=80 \%$ samples. Aluminum concentrations around $x \approx 30 \%$ especially tend 


\begin{tabular}{|c|c|c|c|c|c|c|c|c|}
\hline 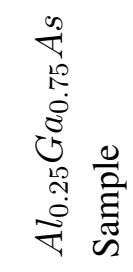 & $\vec{\pi}$ & $\begin{array}{l}\stackrel{\Xi}{\Xi} \\
\stackrel{\tilde{O}}{\circ}\end{array}$ & 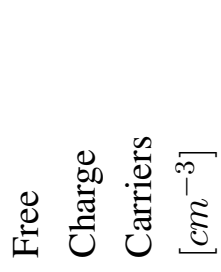 & 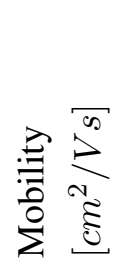 & 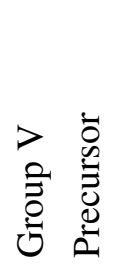 & 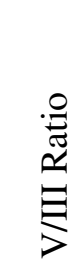 & 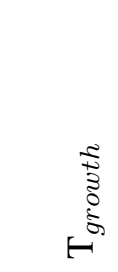 & 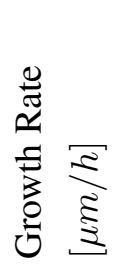 \\
\hline \multirow{2}{*}{ Np4308 } & RT & int. & $p=6 \cdot 10^{17}$ & 107 & TBAs & 21 & $615^{\circ} \mathrm{C}$ & 1.0 \\
\hline & $77 \mathrm{~K}$ & int. & $p=9 \cdot 10^{16}$ & 633 & TBAs & 21 & $615^{\circ} \mathrm{C}$ & 1.0 \\
\hline \multirow{2}{*}{ Np4310 } & RT & int. & $p=3 \cdot 10^{16}$ & 160 & $\mathrm{AsH}_{3}$ & 375 & $615^{\circ} \mathrm{C}$ & 1.0 \\
\hline & $77 \mathrm{~K}$ & int. & $p=3 \cdot 10^{16}$ & 230 & $\mathrm{AsH}_{3}$ & 375 & $615^{\circ} \mathrm{C}$ & 1.0 \\
\hline
\end{tabular}

Table 4.3: Results of Hall-measurements of the nominally undoped samples Np4310 and Np4308 grown with either $\mathrm{AsH}_{3}$ or TBAs, respectively. All other growth parameters are identical ( $\mathrm{T}_{\text {growth }}$ $=615^{\circ} \mathrm{C}, \mathrm{r}_{\text {growth }}=1 \mu \mathrm{m} / \mathrm{h}, 10 \mathrm{~nm}$ GaAs cap) except for the effective surface V/III ratio that depends on the specific thermal decomposition rates. Corresponding surfaces are depicted in 4.13

to increased defect formation for thick layers. Defects grow with increasing layer thickness and show clear faceting. For layers of more than $1 \mu \mathrm{m}$ the loosely scattered defects grow to lateral dimensions of several $\mu \mathrm{m}$ on the surface. This phenomenon is observed especially for $\mathrm{AsH}_{3}$-based growth, despite excellent electrical properties which are comparable with those found in the literature [Cho98]. In contrast, an identical sample grown with TBAs shows a smooth surface. Both sample surfaces are compared in Figure 4.13 The origin of these defects could not be linked to a direct arsenic-precursor contamination.

As a ternary material, $A l G a A s$ needs to form a homogeneous composition without fluctuations for perfect material quality. Due to the very small lattice constant difference between $A l A s$ and $G a A s$, any strain-driven effects are weak. However, literature reports enhanced decomposition behavior for $\mathrm{Al}_{0.3} \mathrm{Ga}_{0.7} \mathrm{As}$ with aluminum-rich/gallium-rich superlattice formation on (111) and (110) surfaces [Har98]. The defects found in our above mentioned $A l_{0.25} G a_{0.75} A s$ samples increase in size but not in number with growing layer thickness. This fixed number of growing defects indicates a common origin from the interface of the structure of all large defects, also for thick layers. Additional experiments showed that defect formation is independent of whether the GaAs buffer layer is grown with TBAs or $\mathrm{AsH}_{3}$. This indicates that the defects do not originate from the wafer-surface/GaAs-buffer interface, but from the GaAs/AlGaAs interface. Consequently it can be assumed that the employed TBAs-based process enables for a superior interface quality compared to $\mathrm{AsH}_{3}$ based growth. Other publications have investigated the GaAs/AlGaAs interface growth problem in more detail concerning QW quality [Mun89, Bim92]. The above shown samples are also different regarding their electrical properties. Both samples are compared in Table 4.3 . Differences are most likely related to 


\begin{tabular}{|c|c|c|c|c|c|c|c|c|}
\hline 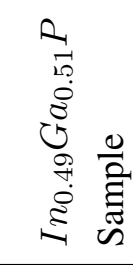 & $\underset{-10}{-3}$ & $\begin{array}{l}\overrightarrow{\tilde{\Xi}} \\
\stackrel{0}{\circ}\end{array}$ & 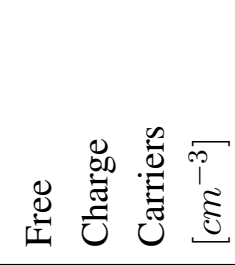 & 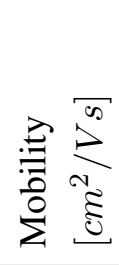 & 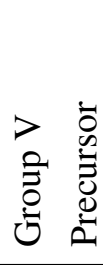 & 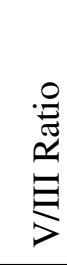 & 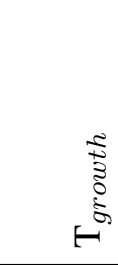 & 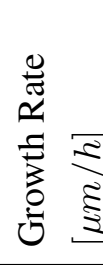 \\
\hline \multirow{2}{*}{ Np2645 } & RT & int. & $n=1.4 \cdot 10^{16}$ & 3370 & TBP & 5 & $615^{\circ} \mathrm{C}$ & 1.4 \\
\hline & $77 \mathrm{~K}$ & int. & $n=8.6 \cdot 10^{15}$ & 20800 & TBP & 5 & $615^{\circ} \mathrm{C}$ & 1.4 \\
\hline Np2647 & RT & $\mathrm{SiH}_{4}$ & $n=8.1 \cdot 10^{18}$ & 480 & TBP & 5 & $615^{\circ} \mathrm{C}$ & 1.4 \\
\hline Np3689 & RT & $\mathrm{DMZn}$ & $p=3.9 \cdot 10^{18}$ & 30 & TBP & 4.4 & $615^{\circ} \mathrm{C}$ & 1.4 \\
\hline
\end{tabular}

Table 4.4: Results of Hall-measurements of selected $\operatorname{In}_{0.49} G a_{0.51} P$ samples lattice matched to GaAs substrate. The table depicts achieved values with corresponding growth parameters.

the variant precursors and are not a consequence of defect formation. $\mathrm{AsH}_{3}$-based growth shows a significantly lower intrinsic hole concentration compared to the TBAs-based sample. Hall data at $77 \mathrm{~K}$ indicates compensated material for the $\mathrm{AsH}_{3}$ sample. In this case, compensation often originates from increased oxygen incorporation, depending on source material quality [Rus03, Kak96].

\subsubsection{InGaP}

In $G a P$ can be grown lattice-matched to GaAs. Direct bandgap energy at $300 \mathrm{~K}$ is $1.9 \mathrm{eV}$ for $I n_{0.49} G a_{0.51} P$, which is about the bandgap energy of $A l_{0.38} G a_{0.62} A s$ [Vur01]. For lattice-matched material at $\lambda=1000 \mathrm{~nm}$ refractive index is $n \approx 3.22$, thus $n$ is equivalent to $A l_{0.55} G a_{0.45} A s$, making $I n_{0.49} G a_{0.51} P$ a suitable alternative cladding layer material for $G a A s$-based devices [Ada85, Tan86, Kan94]. Additionally, $I n_{0.49} G a_{0.51} P$ is ideal in the $A l G a A s$-system as an etchant stopping layer due to its high selectivity for $G a A s$ and $A l G a A s$ [Lee95, Lee00]. Intrinsic properties show low free electron concentrations of $n=1.4 \cdot 10^{16} \mathrm{~cm}^{-3}$ with room temperature carrier mobilities comparable to GaAs. This qualifies that $I n_{0.49} G a_{0.51} P$ is a good alternative cladding material when compared to AlGaAs.

Doping with silicon allows for high doping levels up to $n \approx 1 \cdot 10^{19} \mathrm{~cm}^{-3}$ without visible defect formation. Thus $\mathrm{SiH}_{4}$ is used as dopant precursor for all $n-I n_{0.49} G a_{0.51} P$ samples. Intrinsic doping or doping with tellurium are not investigated.

Initial experiments with carbon doping did not result in significant hole concentrations. Therefore DMZn was installed as an alternative p-dopant source showing very good results. The observed hole concentrations of $p>1 \cdot 10^{18} \mathrm{~cm}^{-3}$ are comparable to the literature [Iwa84]. As lattice matched InGaP has similar crystal properties compared to GaAs, comparable $Z n$ diffusion properties need to also be anticipated. Few reports on diffusion 


\begin{tabular}{llrl} 
Step & Thickness or Time & Temperature & Description \\
\hline \hline 1. & variable & $700^{\circ} \mathrm{C}$ & $G a A s$ layer growth \\
\hline 2. & undefined & to $615^{\circ} \mathrm{C}$ & cool down \\
\hline 3. & $180 \mathrm{~s}$ & $615^{\circ} \mathrm{C}$ & temperature stabilization \\
\hline 4. & $1 \mathrm{~s}$ & $615^{\circ} \mathrm{C}$ & As supply closed \\
\hline 5. & $1 \mathrm{~s}$ & $615^{\circ} \mathrm{C}$ & $\mathrm{P}$ supply open \\
\hline 6. & variable & $615^{\circ} \mathrm{C}$ & $I n_{0.49} G a_{0.51} P$ layer growth \\
\hline 7. & $1 \mathrm{~s}$ & $615^{\circ} \mathrm{C}$ & $\mathrm{P}$ supply closed \\
\hline 8. & $1 \mathrm{~s}$ & $615^{\circ} \mathrm{C}$ & As supply open \\
\hline 9. & $5 \mathrm{~nm}$ & $615^{\circ} \mathrm{C}$ & GaAs capping layer growth \\
\hline 10. & undefined & to $700^{\circ} \mathrm{C}$ & heat up \\
\hline 11. & variable & $700^{\circ} \mathrm{C}$ & further $(A l) G a A s$ growth
\end{tabular}

Table 4.5: Switching sequence for the employed $G a A s / I n G a P$ interface growth process.

of $Z n$ in $I n_{0.49} G a_{0.51} P$ are available [Yoo97, Tak99]. In contrast reports on $Z n$ doping of the corresponding binary compounds $G a P$ and $\operatorname{In} P$ already exist, since high quality single crystal samples are available [Cha64b, Cha64c, Cha64a, Hoo74]. Van Gurp et al. reports that $Z n$ solubility in $I n G a A s P$ is closer to GaAs than to $I n P$, whereas diffusion depth is closer to $I n P$ than in GaAs [vG88]. In general, $Z n$ diffusion in III-V materials is described as an interstitial-substitutional mechanism combined with a "kick-out" of group III atoms [Gös81]. Thus, diffusion coefficients depend on group III vacancy formation energy $E_{f}\left(V_{I I I}\right)$ and the $Z n$ atom migration energy $E_{m}(Z n)$ to move from one group III site to the next. Both energies are calculated to be larger for In GaP than for GaAs. Consequently, $Z n$ diffusion should be suppressed [Tak99]. To minimize any vacancydriven diffusion effects, optimal material quality is required, without defects and with ultra-low $V_{I I I}$ densities.

For large-scale applications the most common phosphorus precursor is $\mathrm{PH}_{3}$. Within this work all $I n G a P$ layers are grown using the less hazardous TBP as a group $\mathrm{V}$ precursor. Hall measurements of selected characteristic samples are presented in Table 4.4. The most crucial parameters for all In GaP layers in the $G a A s$-system are the precisely-controlled composition, to achieve $I n_{0.49} G a_{0.51} P$ material, along with the growth of high-quality $\mathrm{GaAs} /$ In $\mathrm{GaP}$ hetero-interfaces. For such interfaces, a group $\mathrm{V}$ precursor exchange is required. To assure defect-free and sharp hetero-interface growth, an elaborate precursor switching sequence based on short one second flushing steps was employed. A complete description of the switching sequence process parameters is given in Table 4.5 . 



\section{Chapter 5}

\section{Edge-emitting quantum dot lasers}

From an epitaxial point of view, edge-emitting lasers with a SCH design are a wellestablished growth process. However, technological difficulties occur if applications require specially-designed active layers to extend the accessible spectral range for a distinct material system or demand ultra-efficient performance. For $G a A s$-based processes it is especially demanding to produce long wavelength laser emission at $1.3 \mu \mathrm{m}$. To extend the emission wavelength this far not only is the development of high-density QD growth processes for sufficient gain required, but also the suppression of any unintended blue shift during growth of the complete upper half of the edge-emitter design [Le096]. While lower growth temperatures reduce QD emission blue shift, the consequence can be degraded material quality. Thus, the real challenge for efficient low threshold lasers close to 1.3 $\mu \mathrm{m}$ is to maintain a high QD density and excellent material quality, while eliminating any emission blue shift.

\subsection{Broad waveguide lasers}

The versatile design of the broad waveguide laser was chosen to approach the goal of efficient QD lasers at $1.3 \mu \mathrm{m}$, enabling fast access to laser characteristics. This becomes possible by straightforward processing, the so called Schnellprozess, which can be accomplished by well-trained people for first characterization results within two working days. This fast process technology for broad waveguide edge-emitters is detailed in A.5.1 Thus edge-emitting lasers can serve as excellent fast benchmarking tools for the active QD section and for the complete device growth process.

All fabricated QD lasers are based on the integration of efficient, thermally stable QD layers into a complete laser structure. The additional overgrowth process required for a complete laser structure compared to a PL test sample, and the altered starting conditions for QD growth by a slightly different surface quality due to additional buried layers, necessitate a careful growth process optimization for all layers within the laser structure. For electrical charge carrier injection the edge-emitting lasers are designed as pin-structures. All edge-emitting lasers in this work were grown on silicon doped $G a A s$ (001) substrates ( $n \approx 6 \cdot 10^{19} \mathrm{~cm}^{-3}$ ). Bottom and top cladding need to be sufficiently doped for charge carrier injection ( $n$ or $p \approx 1 \cdot 10^{18} \mathrm{~cm}^{-3}$ ), whereas the topmost GaAs contact layer needs 


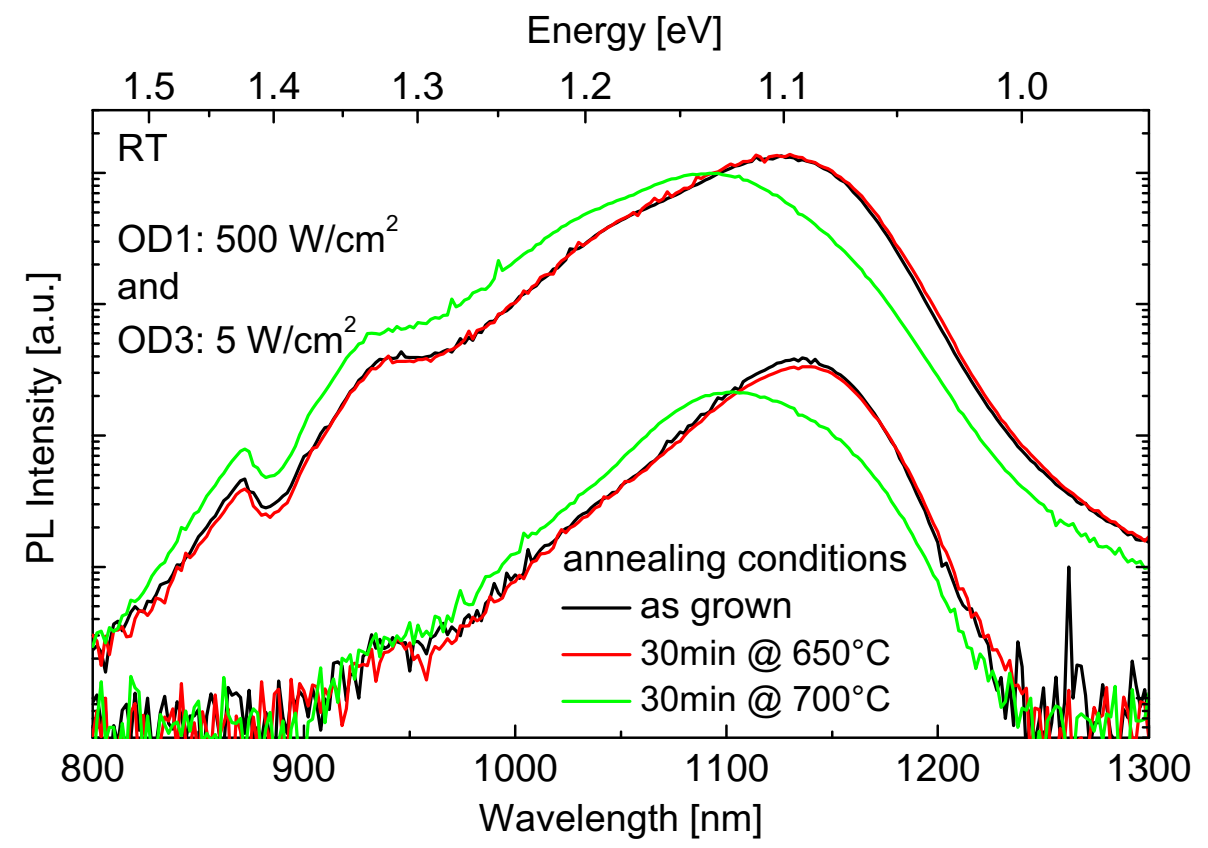

Figure 5.1: Annealing of single PL test sample with three-fold $\operatorname{In}_{0.75} G a_{0.25} A s$ QD stack, 40 $\mathrm{nm}$ spacer and without SRL. Annealing conditions: $30 \mathrm{~min}, 100 \mathrm{mbar}, \mathrm{H}_{2}$ carrier gas, $15 \mathrm{l} / \mathrm{min}$, $22 \cdot 10^{-5} \mathrm{~mol} / \mathrm{min} \mathrm{TBAs}$ flow for surface stabilization. Annealing at $650^{\circ} \mathrm{C}$ shows almost no effect, whereas annealing at $700^{\circ} \mathrm{C}$ causes significant luminescence degradation.

a higher doping level of $p \approx 1 \cdot 10^{19} \mathrm{~cm}^{-3}$ to enable metal contacts with ohmic instead of Schottky-diode-like characteristics. If the employed dopant precursors tend to diffuse into adjacent layers (e. g. Te or $\mathrm{Zn}$ ) specific doping profiles and growth parameters can become necessary to avoid dopant incorporation within the waveguide.

\section{Edge-emitters and thermal stability}

Overgrowth of a QD layer stack can conventionally be seen as an annealing step which depends only on temperature and time. This annealing can have a strong influence on QD properties depending on the type of QDs (i. e. size, indium content, SRL), the spacer thickness, and stacking number [Tat04]. The change in QD luminescence upon annealing is caused by Fickian interdiffusion of indium out of the QDs into surrounding indium free matrix material [Hei98b]. This process results in a reduced density of optically active QDs, QD ensemble broadening, and an unintended wavelength blue shift due to the reduced indium content within the QD layer.

To investigate thermal stability of QDs a three-fold-stacked PL sample has been annealed under different conditions. Note that QDs in this PL sample are already overgrown by $G a A s$ and $A l G a A s$ layers totaling to $\approx 150 \mathrm{~nm}$ prior to the annealing experiment. Con- 


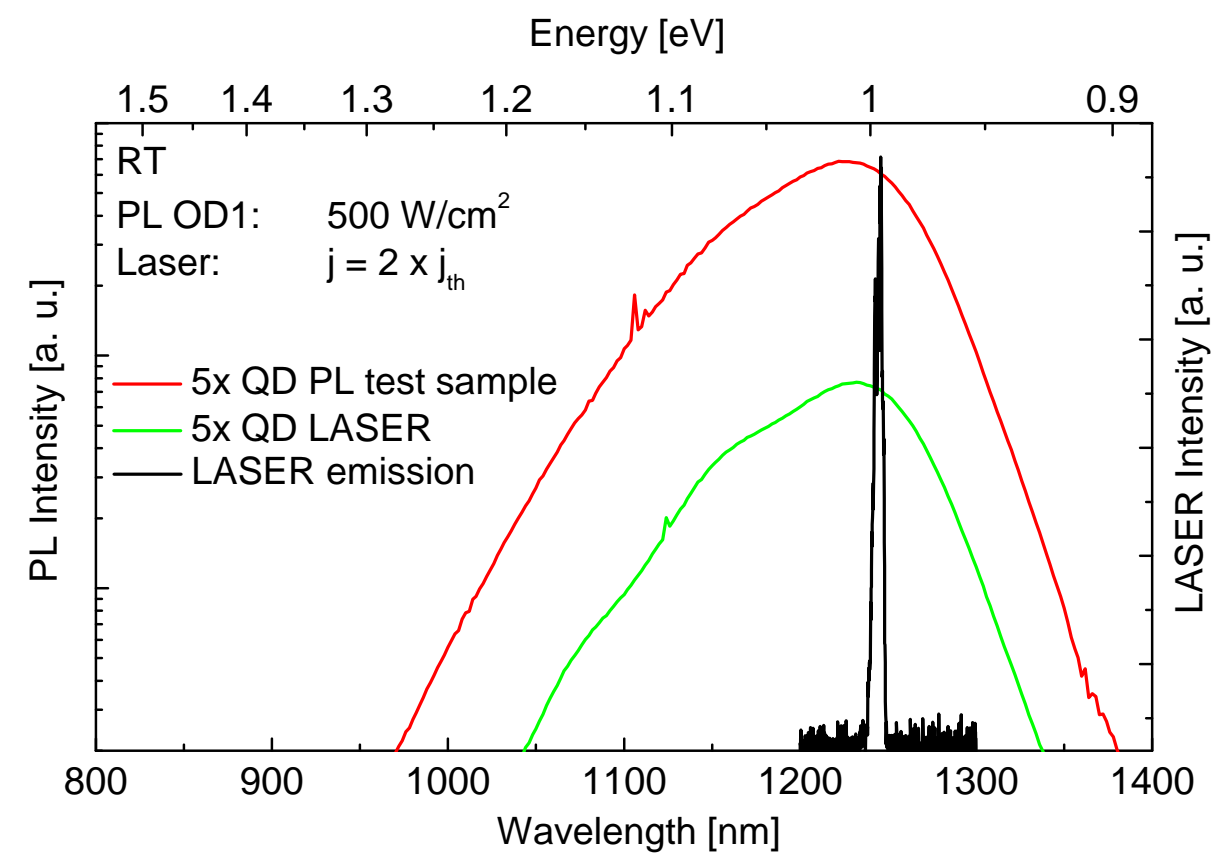

Figure 5.2: Comparison of PL measurements of the optimized PL test sample, the complete laser structure, and with laser emission of the processed final device, including each a five-fold QD stack. Any unintended blue shift is eliminated and lasing mode appears at the GS maximum.

sequently, annealing effects on QDs can only be attributed to segregation effects within the crystal as QDs are already far from the surface. For this experiment, the TBAs stabilization flow, pressure, and total reactor flow were kept constant for both annealing temperatures. Figure 5.1 shows the dependency on annealing temperature, evidenced by a strong blue shift of $34 \mathrm{~nm}$ combined with an integral OD1 luminescence reduction of $10 \%$ at $700^{\circ} \mathrm{C}$. In this case, a temperature reduction to $650^{\circ} \mathrm{C}$ could completely suppress the blue shift and luminescence loss of this QD stack, however these QDs include no SRL and were tuned to a rather short wavelength close to $1.15 \mu \mathrm{m}$. For QDs emitting at longer wavelengths even a temperature reduction to $615^{\circ} \mathrm{C}$ was insufficient, as will be shown later in Figure 5.16 . Consequently, a temperature reduction for subsequent layer growth contributes to blue shift reduction, but was insufficient for the longest wavelength devices, and it is also a tradeoff for optimum defect-free material quality if the temperature becomes too low.

Compared to thinner PL test structures, edge-emitting lasers include additional thick cladding layers to ensure optical confinement within the waveguide with the enclosed active layers. To minimize the influence of cladding layer growth on QD properties, a high aluminum content of $80 \%$ was chosen for the AlGaAs claddings. This enabled a large refractive index contrast between $G a A s$ waveguide and cladding layers. Consequently, thinner p-claddings of only 700 to $800 \mathrm{~nm}$ become possible compared to typical edgeemitter designs with cladding thicknesses of up to $1300 \mathrm{~nm}$ and aluminum contents of 

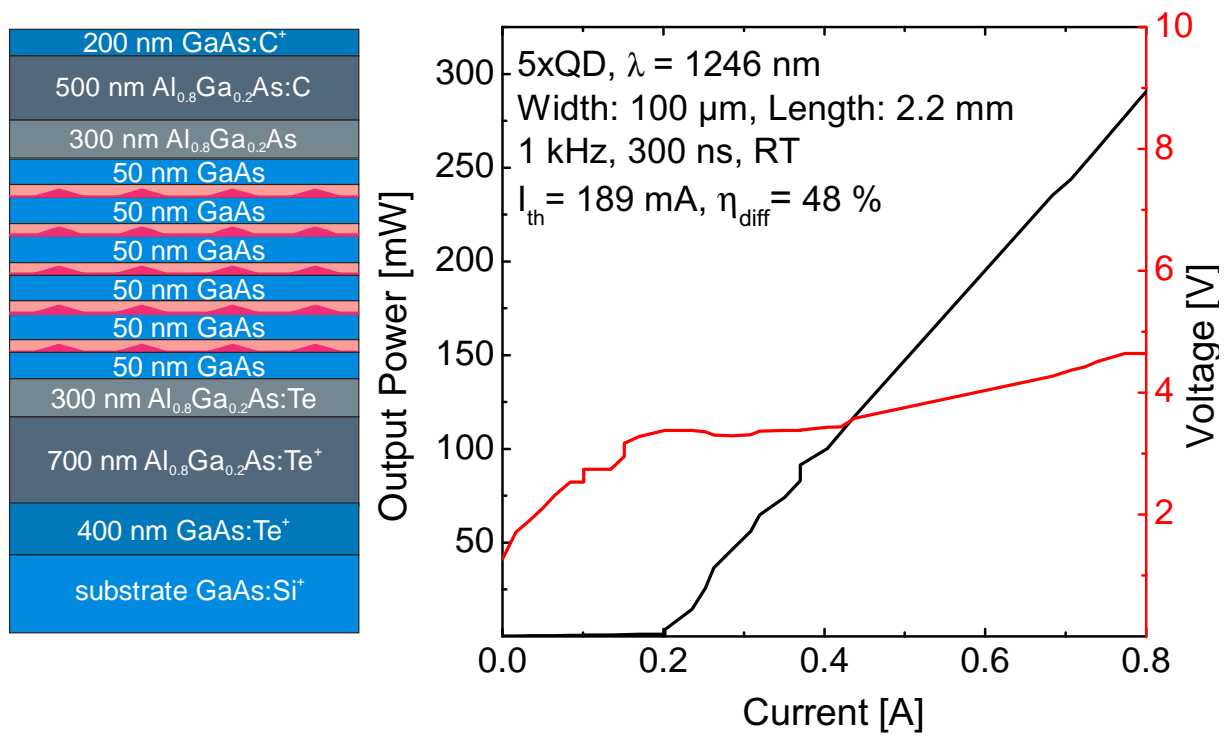

Figure 5.3: left: Schematic of the grown laser structure employing $A l_{0.8} \mathrm{Ga}_{0.2} \mathrm{As}$ claddings with $\mathrm{Te}$ and $\mathrm{C}$ doping and a five-fold QD stack active region. right: L-I-V curves under pulsed injection at room temperature.

60 to $40 \%$ [Kai04, Tat04, Tat05a]. These thinner p-cladding layers on top of the QD active region were grown at a reduced temperature of only $615^{\circ} \mathrm{C}$ at high growth speeds, from $1.5 \mu \mathrm{m} / \mathrm{h}$ to $2.5 \mu \mathrm{m} / \mathrm{h}$, to minimize overgrowth time to $30 \mathrm{~min}$, and thus reducing any possible influence on the buried QD layers. Additionally, a moderate V/III ratio of 28 is chosen for the $A l_{0.8} G a_{0.2} A s$ layers, and the topmost $200 \mathrm{~nm} G a A s$ contact layer is grown with a V/III ratio of 5. Figure 5.2 demonstrates the excellent outcome of this process, even for longer wavelength QDs around $1250 \mathrm{~nm}$. The additional cladding layer growth causes no emission wavelength shift of the final laser structure in comparison with the optimized QDs of the thinner PL test structures. High optical gain from the included five-fold QD stack enables QD ground state laser emission of the final devices at the spectral peak luminescence position of the previously grown PL test structure, eliminating any overgrowth blue shift.

\section{AIGaAs:Te/C edge-emitters}

Based on work of [Kai06], QD growth process optimization as detailed in Chapter 4 and an optimized QD edge-emitter design the above shown blue shift suppression at $1.25 \mu \mathrm{m}$ is enabled combined with significantly improved laser device characteristics. The new 


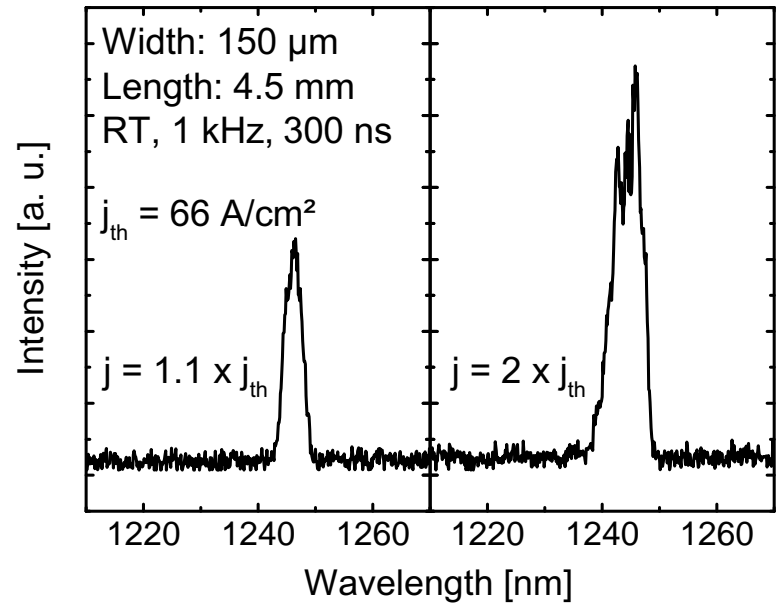

Figure 5.4: Laser emission spectra: left - close to lasing threshold; right - at twice the laser threshold current density.

growth process enabled $1246 \mathrm{~nm}$ lasing with record low $j_{t h}=66 \mathrm{~A} / \mathrm{cm}^{-2}$. These results are already published and were presented at ICMOVPE XIII, Japan [Str06, Ger07].

Laser growth of this design is carried out solely by alternative precursors without $\mathrm{AsH}_{3}$. A schematic of this novel laser design and its characteristic L-I-V curves are presented in Figure 5.3. Emission spectra around threshold and at twice the threshold are shown in Figure 5.4 The doped claddings are realized by $\mathrm{Al}_{0.8} \mathrm{Ga}_{0.2} \mathrm{As}$ layers using the afore described optimized overgrowth process, with a thinner overall design and a low V/III ratio of 28 . In consequence, the overgrowth time could be limited to only $36 \mathrm{~min}$. As shown in 5.2, a complete suppression of emission blue shift upon integration in the complete laser structure is achieved. The laser design employs a stepped doping scheme with doping levels reduced by one order of magnitude within $300 \mathrm{~nm}$ around the waveguide core, minimizing optical losses in the claddings. Hereby the probability of Te migration into the undoped active region is reduced for the bottom $\mathrm{Al}_{0.8} \mathrm{Ga}_{0.2} \mathrm{As}$ : Te cladding. The top $A l_{0.8} G a_{0.2} A s / A l_{0.8} G a_{0.2} A s: C$ cladding employs $C B r_{4}$ as carbon source only for the upper, highly $p=5 \cdot 10^{18} \mathrm{~cm}^{-3}$ doped part, while the lower part is set to $p=5 \cdot 10^{17} \mathrm{~cm}^{-3}$ by intrinsic carbon doping. This design compromise allows for sufficient charge carrier transport while limiting optical losses due to high dopant concentrations.

The undoped $G a A s$ waveguide core encloses five active QD layers separated by $50 \mathrm{~nm}$ GaAs spacers. The growth temperature is raised from $515^{\circ} \mathrm{C}$ to $615^{\circ} \mathrm{C}$ after capping the QD layer with GaAs to achieve optimal GaAs matrix material quality. Capping thickness is $\approx 7 \mathrm{~nm}$ before and $\approx 4 \mathrm{~nm}$ during heating. After every heating step the sample is annealed $30 \mathrm{~s}$ at $615^{\circ} \mathrm{C}$ to flatten the surface before spacer growth. Thus growth of high quality spacer layers becomes possible without affecting the buried QDs. Total dimension of the waveguide core is $\approx 300 \mathrm{~nm}$.

A $1246 \mathrm{~nm}$ emission wavelength is achieved with $I n_{0.65} G a_{0.35} A s$ QDs and a $I n_{0.08} G a_{0.92} A s$ SRL. Stacking quality and optical gain are significantly improved by 
Figure 5.5: An extremely low transparency current density of $j_{t r}=52$ $\mathrm{A} / \mathrm{cm}^{2}$ is deduced from a set of lasers with cavity lengths up to $8 \mathrm{~mm}$.

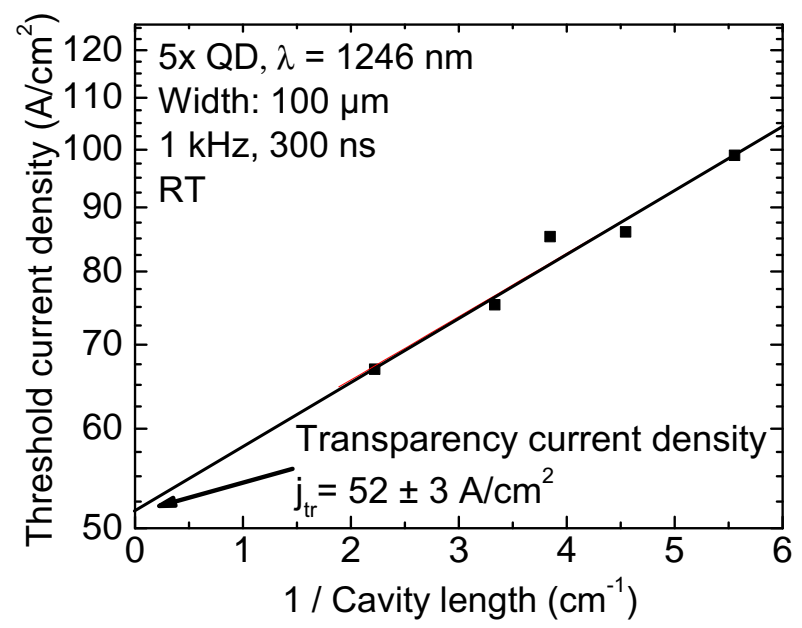

individual growth interruption times for the QD layers (cf. 4.1.2). A series with 3, 4, and 5 QD layers showed that a five-fold QD stack is required to achieve sufficiently high optical gain for ground state lasing. The results of this improved laser design surpassed previous best values for our group with a ten-fold QD laser $\left(j_{t h}=220 \mathrm{~A} / \mathrm{cm}^{-2}, \eta_{\text {int }}=74 \%\right.$ at $\lambda=1243 \mathrm{~nm}$ ) [Kai04]. Figure 5.5 shows the transparency current density which could be reduced by $28 \%$ from $j_{t r}=72 \mathrm{~A} / \mathrm{cm}^{2}$ to $j_{t r}=52 \mathrm{~A} / \mathrm{cm}^{2}$ due to the minimized number of QD layers. The excellent internal efficiency of close to $\eta_{\text {int }}=100 \%$ is shown in Figure 5.6. whereas internal losses are still significant with $\alpha_{i}=6 \mathrm{~cm}^{-1}$. Optimized graded interfaces between core $\mathrm{GaAs}$ and cladding $\mathrm{Al}_{0.8} \mathrm{Ga}_{0.2} \mathrm{As}$ layers could likely reduce losses. Epitaxial problems from $\mathrm{GaAs} / \mathrm{AlGaAs}$ interfaces have been intensely investigated in the past for QW structures [Mun89, Bim92]. Another important loss mechanism is free-carrier scattering by electrons and holes, which is especially relevant due to the high intrinsic carbon incorporation of $A l G a A s$, whereas QD scattering losses are small [Smo01]. Despite

Figure 5.6: Internal efficiency comes close to $100 \%$, whereas significant internal losses of $\alpha_{i}=6$ $\mathrm{cm}^{-1}$ are measured. Losses are mainly attributed to optical loss at the GaAs/AlGaAs cladding interfaces and free charge carriers.

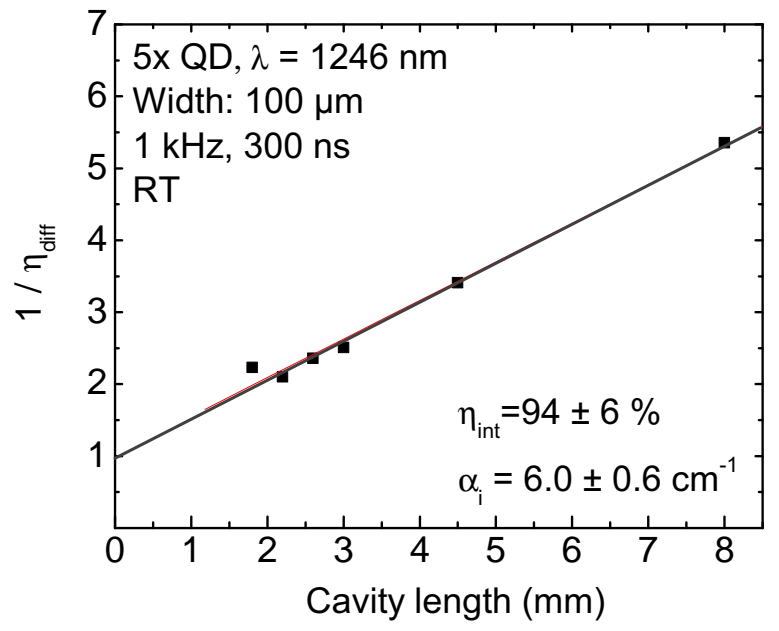




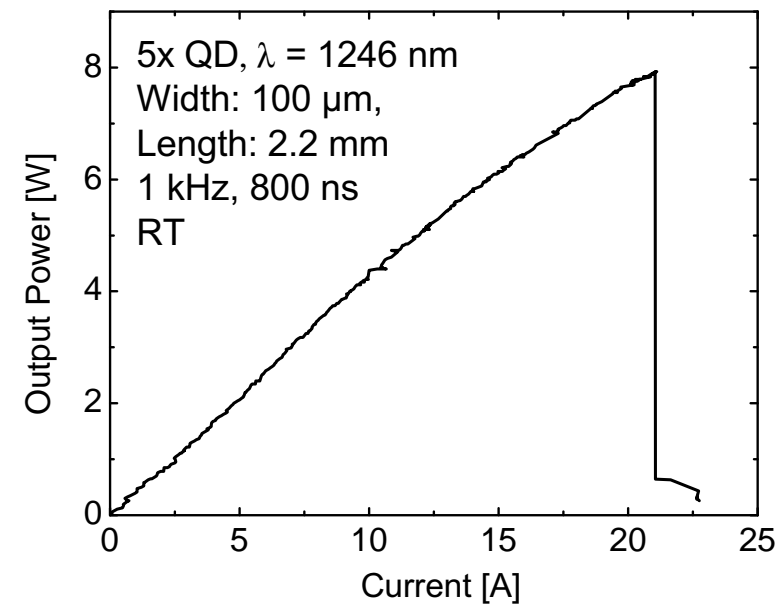

Figure 5.7: Up to $8 \mathrm{~W}$ optical output power at $1246 \mathrm{~nm}$ in pulsed lasing operation is achieved. The measured laser diode is part of a complete multiple laser bar and not mounted to a heat sink.

the internal losses, up to $8 \mathrm{~W}$ optical output power was achieved in pulsed mode (cf. Figure 5.7 , along with an ultra-low threshold current density of $j_{t h}=66 \mathrm{~A} / \mathrm{cm}^{-2}$. Operation at $73^{\circ} \mathrm{C}$ allowed for $\lambda=1263 \mathrm{~nm}$ with $j_{t h}=163 \mathrm{~A} / \mathrm{cm}^{-2}$. Higher temperatures were limited by the setup. The epitaxial growth showed an excellent homogeneity on the whole wafer, enabling up to $8 \mathrm{~mm}$ long devices.

\section{AIGaAs:Si/C edge-emitters}

To eliminate the memory effect of tellurium doping, a $\mathrm{SiH}_{4}$ line was added to the MOVPE system as an alternate n-dopant source. As detailed in 4.2, $\mathrm{SiH}_{4}$ is limited for $A l_{0.8} G a_{0.2} A s$ to lower n-doping levels compared to tellurium. Using TBAs the growth temperature could be reduced from $700^{\circ} \mathrm{C}$ to $615^{\circ} \mathrm{C}$ for the bottom $A l_{0.8} G a_{0.2} A s$ : Si to minimize carbon incorporation. Despite inferior quality of the employed TMAl source an n-doping level of $n=2 \cdot 10^{17} \mathrm{~cm}^{-3}$ was achieved. Due to this lowered doping level, no

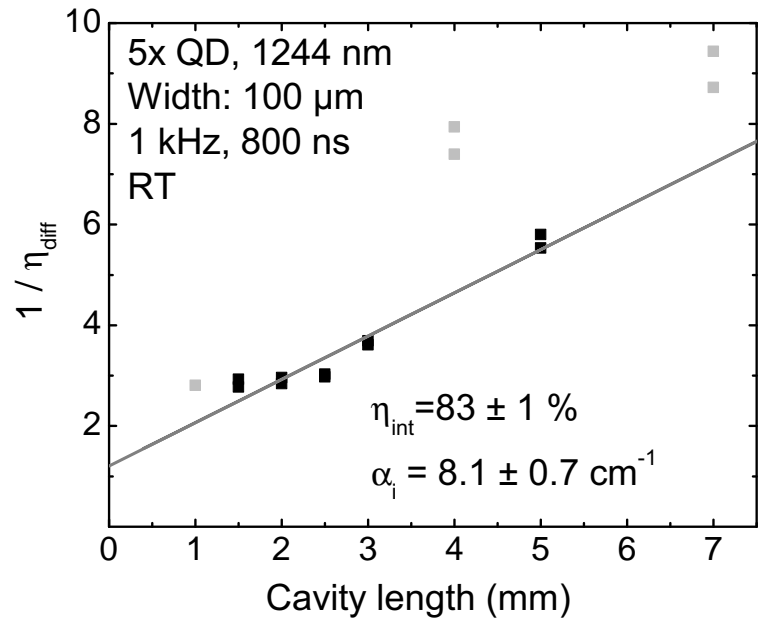

Figure 5.8: Slightly inferior internal efficiency of $\eta_{\text {int }}=83 \%$ compared to the tellurium doped device still represents a very good value. 

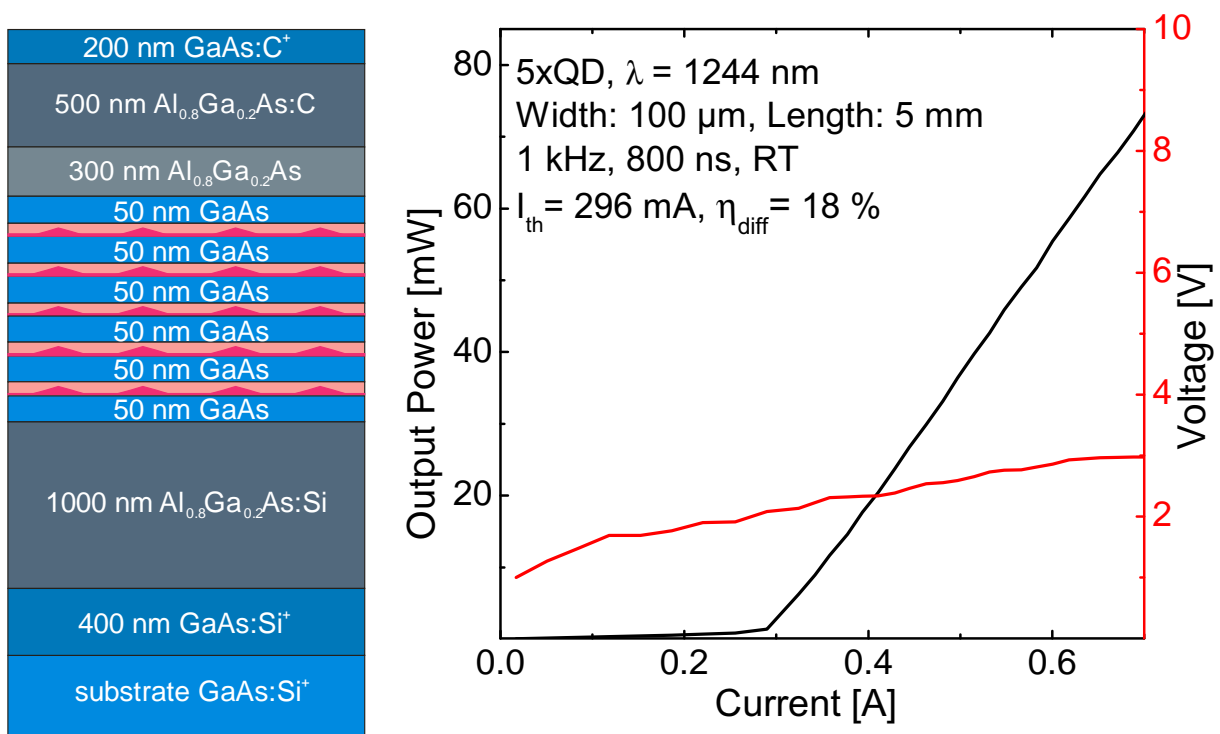

Figure 5.9: left: Schematic of the grown laser structure employing $A l_{0.8} \mathrm{Ga}_{0.2} \mathrm{As}$ claddings with $\mathrm{Si}$ and $\mathrm{C}$ doping and a five-fold $\mathrm{QD}$ stack active region. right: $\mathrm{L}-\mathrm{I}-\mathrm{V}$ curves under pulsed injection at room temperature.

stepped doping scheme was employed for the bottom-cladding layer. Additionally, the cladding layer growth rates were increased from $\approx 1.6 \mu \mathrm{m} / \mathrm{h}$ to $\approx 2.5 \mu \mathrm{m} / \mathrm{h}$ to reduce the overgrowth time from $36 \mathrm{~min}$ to only $25 \mathrm{~min}$ while maintaining good material quality. Precursor efficiency of the cladding layers was improved by reducing the total reactor flow rate from $15 \mathrm{l} / \mathrm{min}$ to $10 \mathrm{l} / \mathrm{min}$, leading to 1.7 -fold growth rates. However, characterization of multiple laser samples across the wafer showed an inferior homogeneity compared to

Figure 5.10: Transparency current density of $j_{t r}=40 \mathrm{~A} / \mathrm{cm}^{2}$ is significantly reduced compared to the tellurium doped device. Giving an excellent value of $j_{t r}=8 \mathrm{~A} / \mathrm{cm}^{2}$ per QD layer.

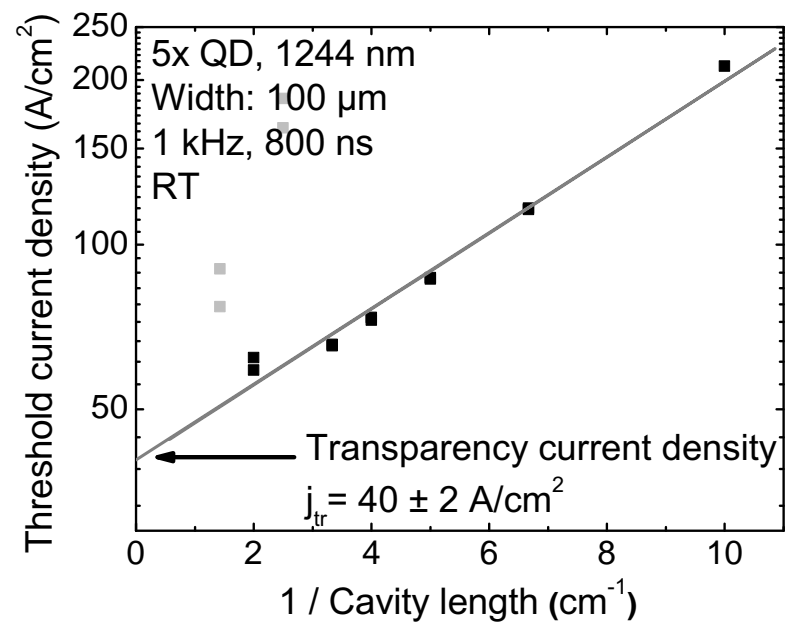


the $15 \mathrm{l} / \mathrm{min}$ process. The employed QDs are based on the same process with $15 \mathrm{l} / \mathrm{min}$ total flow as detailed for the $A l G a A s: T e / C$ edge-emitter in 5.1. Increased internal losses of $\alpha_{i}=8.1 \mathrm{~cm}^{-1}$ were most likely caused by higher intrinsic p-doping of the $A l_{0.8} G a_{0.2} \mathrm{As}$ layers due to a new replacement TMAl source, while internal efficiency remained very good with $\eta_{i n t}=83 \%$ (cf. Figure 5.8). Laser characteristics and a schematic of the layer structure are given in Figure 5.9. The device showed laser emission at a similar wavelength of $1244 \mathrm{~nm}$, and a further reduced threshold current density of $j_{\text {th }}=60 \mathrm{~A} / \mathrm{cm}^{-2}$ at RT was measured. The transparency current density was significantly reduced to only $j_{t r}=40 \mathrm{~A} / \mathrm{cm}^{2}$ giving an excellent value of $j_{t r}=8 \mathrm{~A} / \mathrm{cm}^{2}$ per QD layer (cf. Figure 5.10).

\subsubsection{Aluminum-free laser design}

Aluminum is a very reactive element which forms $\mathrm{Al}_{2} \mathrm{O}_{3}$ oxide in contact with air. All free $A l(G a) A s$ surfaces of a structure oxidize, preventing further growth on these surfaces and causing enhanced optical losses in laser waveguides. Narrow waveguide lasers with $A l(G a) A s$ claddings are required to include additional sidewall passivation, which is not necessary for InGaP-based devices [Kim04]. Thus aluminum-free structures are advantageous for edge-emitters but are also a prerequisite for optical amplifiers, which include a taper process with a second epitaxial growth process on the etched structure. As an alternative to conventional $A l G a A s$ cladding layers, lattice matched $I n_{0.48} G a_{0.52} P$ was investigated. The epitaxial challenges include the control of perfectly lattice matched ternary composition to grow strain-free material on $\mathrm{GaAs}$, realization of sufficiently high $\mathrm{n}$ - and p-doping levels, and most importantly growth of defect-free interfaces, as detailed previously in Table 4.5 .

\section{InGaP:Si/Zn edge-emitters}

The basic design of the $\operatorname{In} G a P: S i / Z n$ edge-emitters was based on the low threshold process of the $A l G a A s: S i / C$ lasers. Since carbon-based p-doping is not possible for $I n_{0.48} G a_{0.52} P$, zinc from a DMZn source was used instead. All employed intrinsic and intentional doping levels are detailed in Table 4.4. New insights into the influence of the V/III ratio on QD properties, as described in Subsection 4.1.2, were used for spacer growth. Layers following QD deposition were grown by stepwise increased V/III ratios of 2, 4.5, and 8 to achieve optimum spacer material quality. This enables for $10 \%$ closer distance (45 nm) of stacked QDs, which in consequence improves the confinement factor. To ensure spectral stability of the buried QDs, all upper layers were grown with a minimum V/III ratio. Good $I n_{0.48} G a_{0.52} P$ cladding material quality with TBP was achieved at a V/III ratio of only 6 at $615^{\circ} \mathrm{C}$. The final $G a A s$ contact layer was grown with a V/III ratio of 6 . 

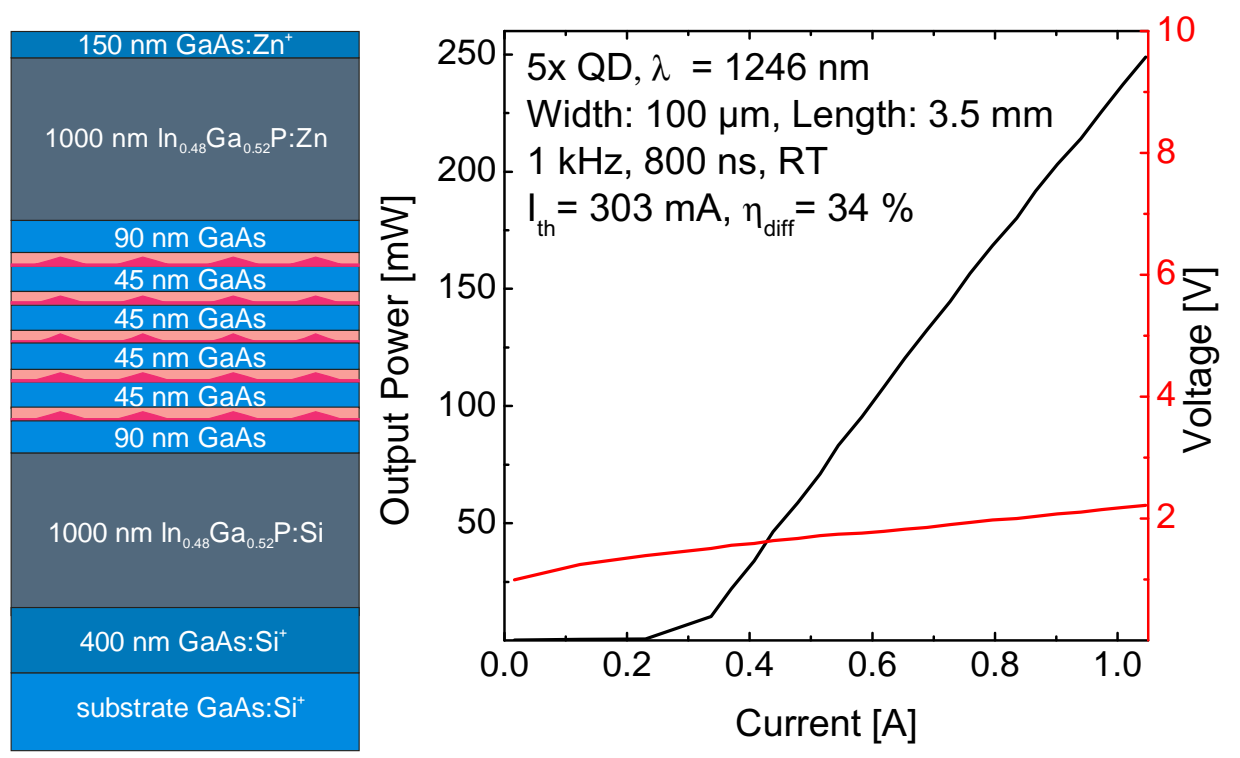

Figure 5.11: left: Layer structure schematic of the grown aluminum-free laser employing $I n_{0.48} G a_{0.52} P$ claddings with $\mathrm{Si}$ and $\mathrm{Zn}$ doping. The five-fold QD stack active region is realized with the optimized thinner spacers. right: L-I-V curves under pulsed injection at room temperature.

Most of the grown wafer was designed for further processing and overgrowth to realize QD-based optical amplifiers, and was given to our partners at the Heinrich Hertz Institute. Only an edge piece was processed employing the Schnellprozess at TU Berlin as edgeemitters to assess sample quality. The processed lasers showed some inhomogeneities for lasers with a length of $5 \mathrm{~mm}$ or more, which was attributed to their origin from the

Figure 5.12: Losses are reduced to $\alpha_{i}=4.8 \mathrm{~cm}^{-1}$, indicating good $\mathrm{GaAs} / \mathrm{In}_{0.48} G a_{0.52} \mathrm{P}$ interface quality. Longer devices ( $5 \mathrm{~mm}$ or more) show deviating data, indicating inhomogeneous material due to the processed border part of the wafer, and are excluded from the fit. In consequence, the measurement error is significant for the internal efficiency of $\eta_{i n t}=77 \% \pm 20 \%$.

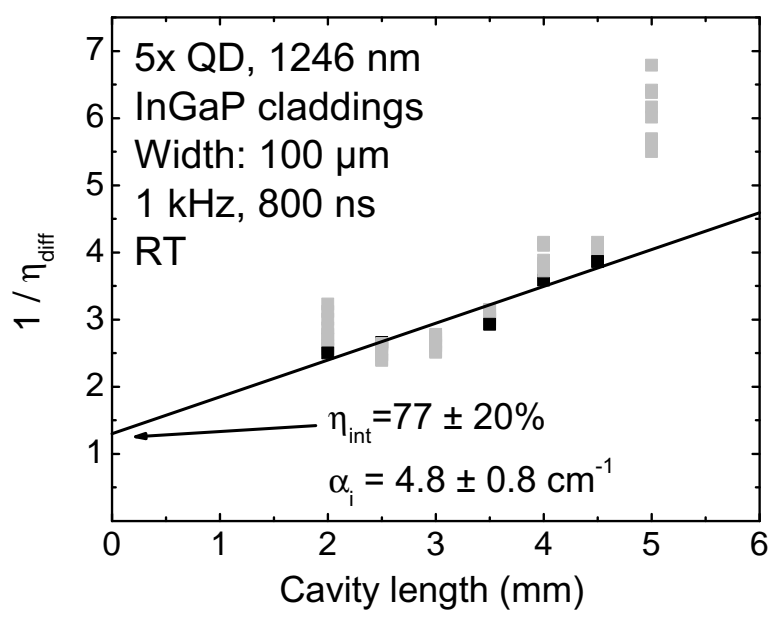




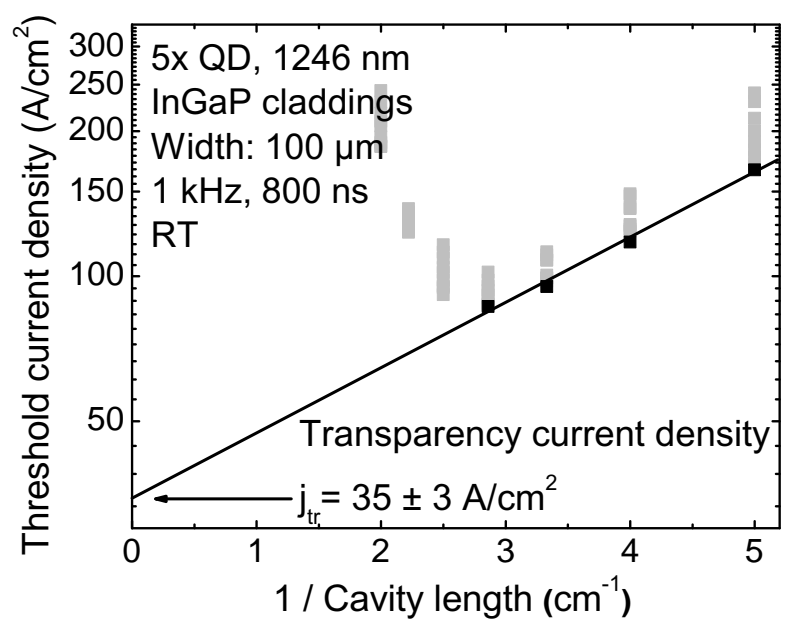

Figure 5.13: Transparency current density is further reduced to $j_{t r}=35$ $\mathrm{A} / \mathrm{cm}^{2}$ setting a new record value for our edge-emitters. Larger devices are excluded for the fit due to inhomogeneities of the processed sample from the wafer border.

wafer border. A schematic of the design and laser characteristics are given in Figure 5.11 Nevertheless, fabricated devices from this aluminum-free edge-emitter prototype with optimized QD stack growth process showed excellent laser performance at $1246 \mathrm{~nm}$, as detailed in Figures 5.12 and 5.13 . Transparency current density was further reduced to only $j_{t r}=35 \mathrm{~A} / \mathrm{cm}^{2}$, compared to AlGaAs-based devices, while internal efficiency could only be estimated to $\eta_{\text {int }}=77 \pm 20 \%$ due to inhomogeneities for longer devices. This improved ultra-low $j_{t r}$ value is attributed to the beneficial qualities of $I n_{0.48} G a_{0.52} P$ claddings and the improved QD stacking process. The reduced internal losses of $\alpha_{i}=4.8 \mathrm{~cm}^{-1}$ are another significant improvement attributed to the superior interface and material quality of the $I n_{0.48} G a_{0.52} P$ layers. The threshold current density of only $j_{t h}=87 \mathrm{~A} / \mathrm{cm}^{-2}$ at RT is a best value when compared to literature for QD-based devices with InGaP claddings grown by MBE or MOVPE [Yeh02, Kim04, Cha04]. These excellent results were achieved by an improved closer stacking process and high quality $I n_{0.48} G a_{0.52} P$ cladding growth, enabling for ground state laser emission.

Based on this initial structure and continuative samples, the collaborative project OptiDot between the Heinrich Hertz Institute and TU Berlin developed a successful taper process for QD based optical amplifiers, which is detailed in the diploma-thesis of Gang Lou. 


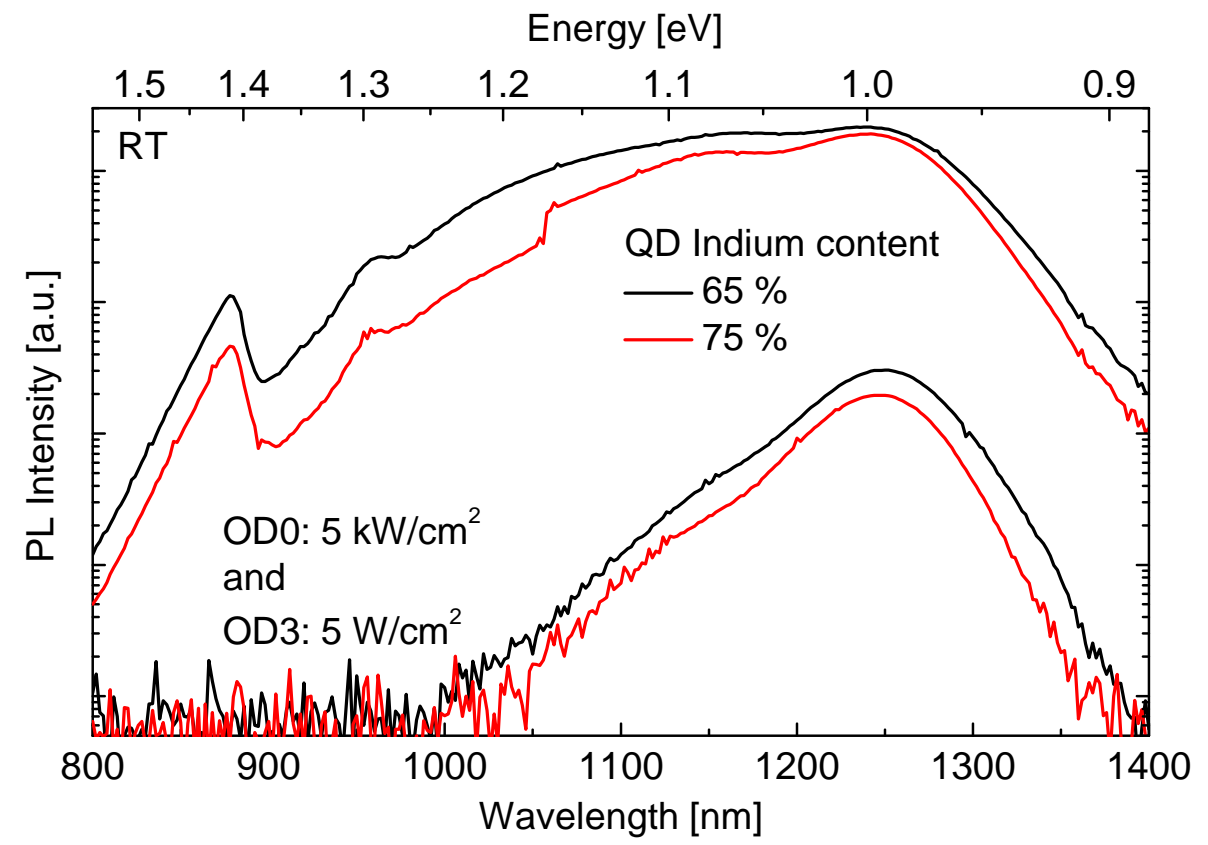

Figure 5.14: PL measurements of samples containing a three-fold InGaAs QD stack with 45 nm spacers. QD layers include an $I n_{0.08} G a_{0.92} A s$ SRL. QD composition is changed from $I n_{0.65} G a_{0.35}$ As to $I n_{0.75} G a_{0.25}$ As resulting in a $32 \%$ integral OD0 luminescence loss and an almost unchanged emission wavelength.

\subsection{Laser diodes at $1.3 \mu \mathrm{m}$}

In order to achieve emission around $1.3 \mu \mathrm{m}$ an increased indium content for the QD layers is generally proposed. For structures with a single QD layer this is an effective method to tune the emission wavelength. Either the QD composition (cf. Figure 4.2) or the indium content of the SRL can be altered (cf. Figure 4.5). However, stacking of such QD layers to increase the total gain becomes increasingly difficult and limits the maximum stacking height due to multiple factors:

- Increased strain per layer promotes the formation of defects such as optically inactive dislocation clusters.

- Larger dots are formed leading to stronger surface corrugation, rendering the optimal integration of QDs into the surrounding GaAs matrix more difficult.

- Ripening of larger dots for long wavelength emission leads to a reduced QD density, reducing the gain per active layer [Pöt04].

- Increased indium content leads to steeper indium gradients, promoting indium diffusion out of the QD layer. 


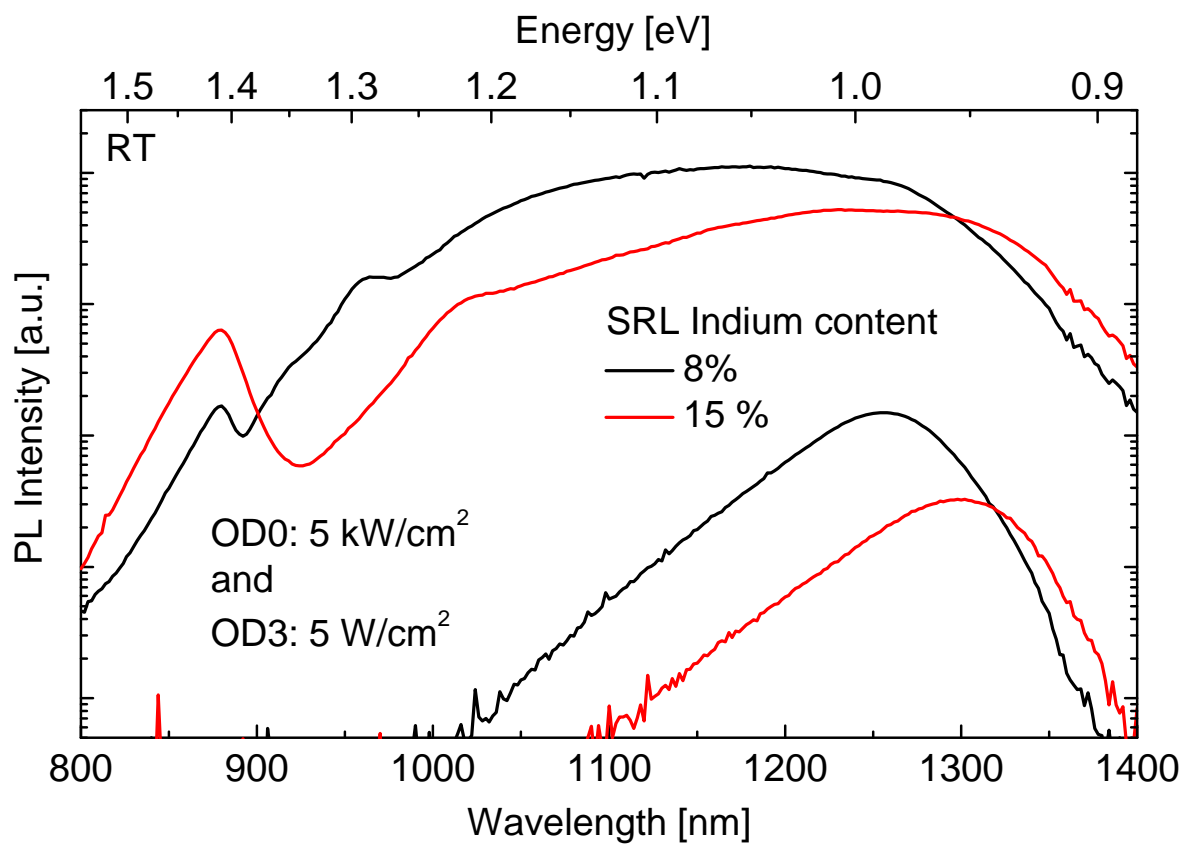

Figure 5.15: PL measurements of samples containing a three-fold $I n_{0.65} G a_{0.35} A s$ QD layer stack with $45 \mathrm{~nm}$ spacers. The SRL composition of the QD layers is varied from $I n_{0.08} G a_{0.92} A s$ to $I n_{0.15} G a_{0.85} A s$, resulting in a strong $44 \mathrm{~nm}$ red shift and a $55 \%$ decrease in integral OD0 luminescence.

The negative effects of increased indium content within QD layers are especially visible for stacked PL samples. Due to the above listed effects all subsequent layers are affected, resulting in significant PL changes. Figure 5.14 shows the influence of a $10 \%$ compositional change of three-fold stacked long wavelength In GaAs QDs. No change in the ground state emission wavelength was observed, while integral luminescence decreased significantly by $32 \%$ at the high excitation density. Regarding low excitation density peak luminescence, the measurement shows a much stronger effect - losing $36 \%$ of its initial luminescence compared to the high excitation density curve with minus $11 \%$. This indicates an increased formation of saturable defects in the proximity of the active layers for the higher indium content sample. Figure 5.15 shows the same experiment but for an indium concentration increase within the SRL from $8 \%$ to $15 \%$. Significant $44 \mathrm{~nm}$ red shift of the QD luminescence was observed combined with a dramatic $77 \%$ decrease in the integral luminescence at low excitation density, indicating the increased formation of defects acting as non-radiative recombination centers. At the high excitation density, luminescence dropped by 55\%, evidencing a strong decrease in the total number of optically-active QDs within the stacked structure.

This dramatic luminescence reduction in both experiments is explained by the fact that all above listed problems already apply for the first QD layer, but all subsequently grown 


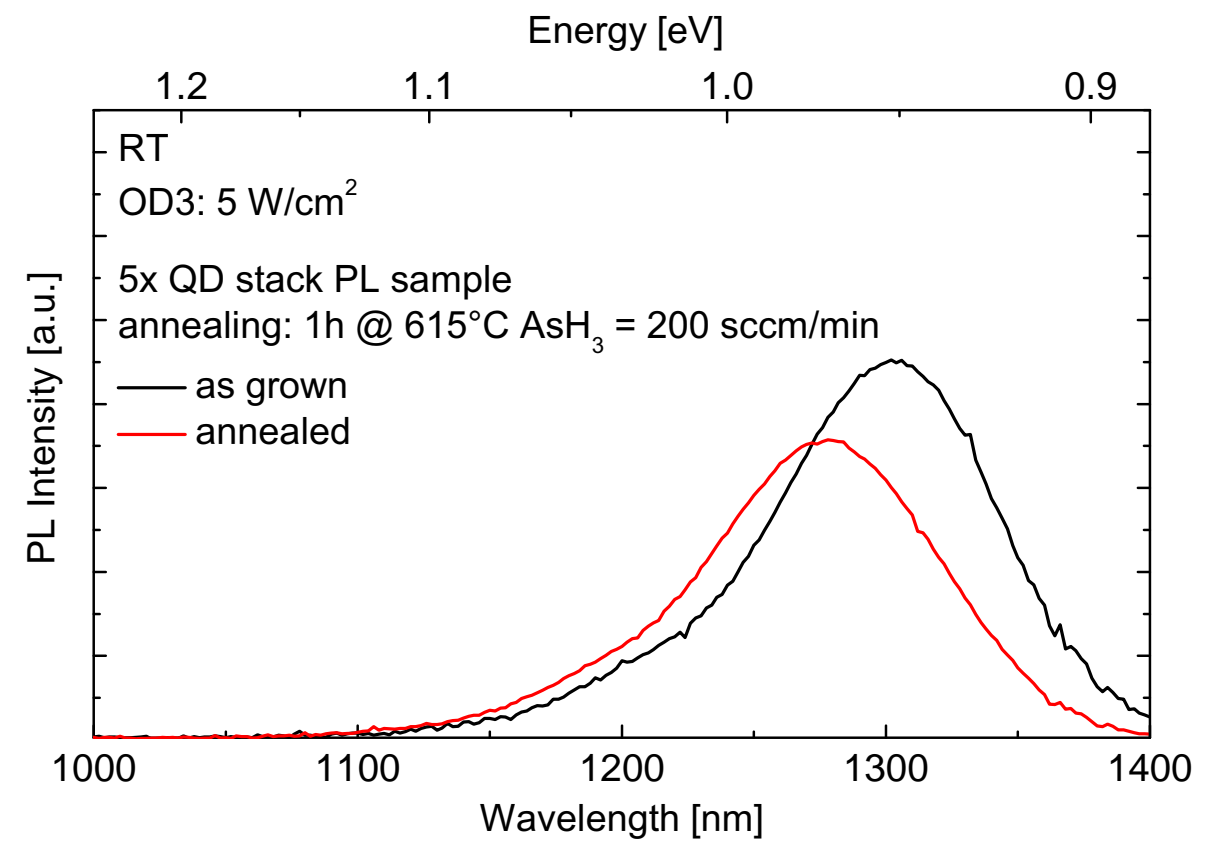

Figure 5.16: PL measurements of a sample containing a five-fold InGaAs QD stack as it was grown and annealed. The sample is not optimized for thermal stability and exhibits $\mathrm{a} \approx 30 \mathrm{~nm}$ blue shift upon $1 \mathrm{~h}$ annealing at $615^{\circ} \mathrm{C}$.

QD layers within the stack suffer additionally from the deteriorated surface conditions, as no strain and defect-free surface was recovered during spacer growth. In consequence, strain and defects of dislocation clusters propagate in the growth direction, degrading subsequent QD layers [Gra10]. Thus, all following layers exhibit an inferior optical quality with less optically active QDs due to a steadily increasing number of dislocation clusters acting as non-radiative recombination centers. Comparison of high and low excitation PL measurements from Figures 5.14 and 5.15 show a stronger luminescence decrease for lowthan for high-excitation densities, indicating the enhanced formation of saturable defects such as point defects within the QD stack for increased indium content. These defects support indium segregation, reducing thermal stability upon overgrowth and annealing. Figure 5.16 shows the effect of $1 \mathrm{~h}$ annealing at the typical growth temperature of top cladding layers $\left(615^{\circ} \mathrm{C}\right)$ on a 5 -fold $1.3 \mu \mathrm{m}$ QD stack. The achieved long wavelength emission was lost due to the insufficient thermal stability, resulting in an unintended blue shift of close to $30 \mathrm{~nm}$. Thus PL data at the desired target wavelength does not indicate thermal stability. Consequently, required supplementary QD growth process optimization is crucial to suppress emission blue shift upon overgrowth and annealing. 


\subsubsection{QD blue shift suppression}

Emission blue shift for laser applications needs to be differentiated between two separate types of origin. One effect is based on insufficient GS gain to achieve lasing threshold, and thus optimized stacking for sufficiently high modal peak gain is crucial to prevent for blue shifted ES laser operation. The second more problematic effect is based on physical changes of the active QD layers due to indium segregation by interdiffusion processes [Leo96, Bab01]. This indium migration out of the QD layers is mainly governed by the vacancy density and is supported by the incorporated strain [Khr97, Ryu95]. In consequence, long wavelength laser applications around $1.3 \mu \mathrm{m}$ are highly affected due to the employed higher indium contents and thus higher total strain [Tat05b]. As GS lasing requires efficiently stacked QD layers with uniform spectral emission, the suppression of wavelength blue shift upon overgrowth is especially demanding. The overgrowth-based blue shift can be simulated by annealing experiments or intentionally used to tune QD emission [Ma197]. Research conducted by other groups to suppress wavelength blue shift is based on modifying vacancy mobility or inhibiting vacancy-based interdiffusion. Such experiments showed that dielectric layers applied prior to annealing of a sample can significantly increase or suppress wavelength blue shift depending on metallurgical reactions and the generated strain due to differing thermal expansion coefficients at the interface [Fu03]. A method which is directly integrated into the QD growth process is based on the use of antimony as a surfactant, either applied on the QD layer or as a $G a A s S b$ buffer layer [Sun05, Ban10]. By inhibiting interdiffusion of indium into the surrounding matrix material and influencing adatom mobilities, this method is used to achieve more uniform QD ensembles and long wavelength emission [Gui06a, Gui06b]. However this method can cause antimony segregation and introduce additional non-radiative defects, making additional growth steps necessary to remove superfluous antimony [Gui09]. Nevertheless, it has been successfully applied to realize laser devices in the $1.3 \mu \mathrm{m}$ range [Gui07, Gui08].

Opposite to the introduction of additional impurities to deal with the effects of high vacancy concentrations, our approach aims at the reduction of the vacancy density in the vicinity of the QD layers at the moment they are formed. In consequence, the low temperature growth steps necessary for QD formation were intensely investigated to achieve complete blue shift suppression by perfection of the grown crystal. In a series of growth experiments we analyzed growth parameters influencing the stability of the QD emission wavelength and intensity upon stacking and overgrowth. Optimization of stacking quality was assessed by stacked QD samples with different numbers of active layers in a constant Matrix volume (cf. Subsection 3.3). Subsequent annealing at overgrowth temperature in the MOVPE reactor simulates thermal stability during overgrowth.

The main optimized QD parameters for defect reduction based on this analysis are the deposition times (amount of material) and the V/III ratios of the InGaAs-QD layer, 


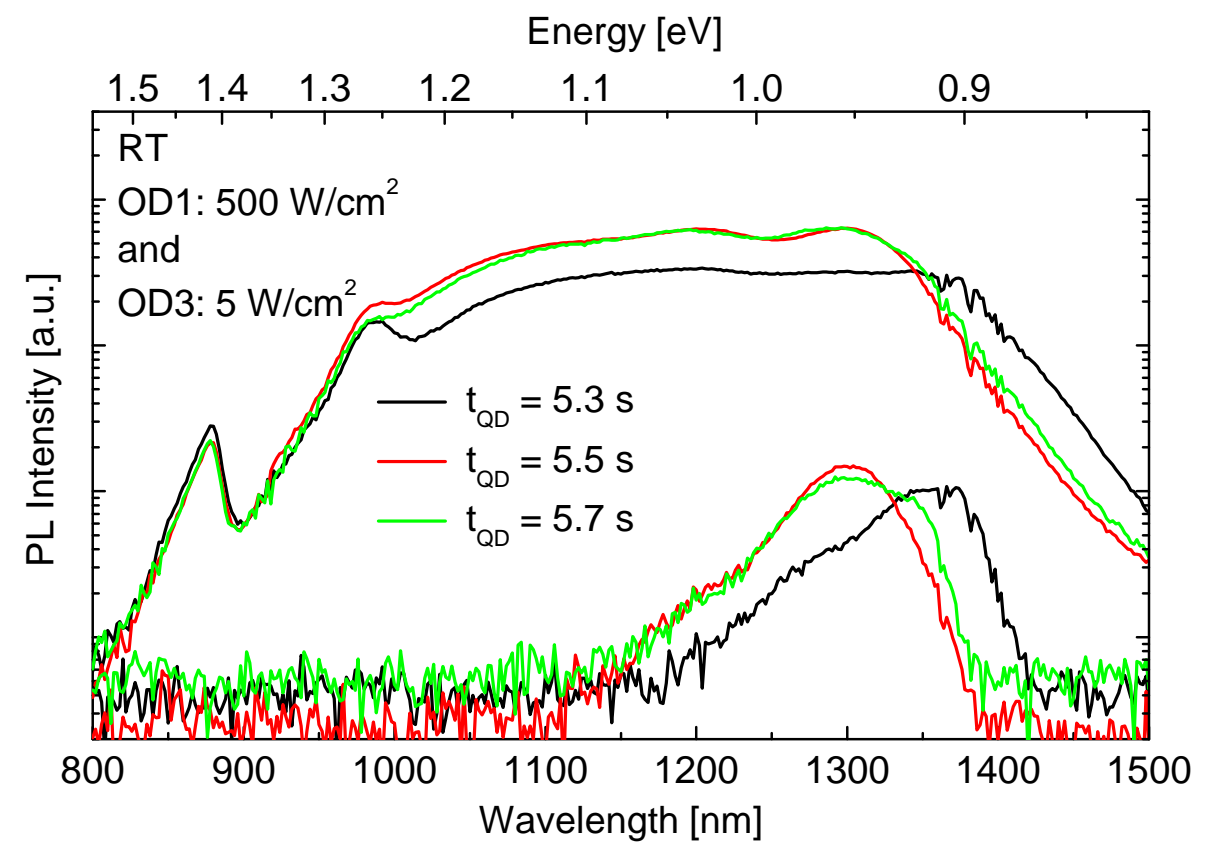

Figure 5.17: PL measurements of samples containing a single InGaAs QD layer and a SRL. QD-material quantity is varied by altering the deposition time $t_{Q D}$ in $0.2 \mathrm{~s}$ steps. Best data is measured for $t_{Q D}=5.5 \mathrm{~s}$.

InGaAs-SRL and low temperature GaAs (LT-GaAs) capping layer prior to heating. These layers are of crucial importance for the final optical properties. As a typical example the variation of the QD deposition time $t_{Q D}$ is shown in Figure 5.17. For these large QDs emitting at $1.3 \mu \mathrm{m}$, a $t_{Q D}$ change of $0.2 \mathrm{~s}$ already results in significant changes in the FWHM and emission wavelength, while the nominal change of deposited material is only $\approx 0.1 \mathrm{ML}$. Thus small variations of the employed very low V/III ratios become significant as growth rates are affected. Consequently, layer thicknesses and compositions change depending on the V/III ratio. Thus, all these parameters are able to cross-influence one another, generating a large parameter space with minimal deviation tolerance.

As layer thickness and V/III ratio both show significant effects for the QD growth process, they need to be optimized with respect to each other. Figure 5.18 demonstrates the influence of the LT-GaAs cap thickness on PL luminescence. The optimum is found at 4.4 nm cap thickness, where the PL intensity is $10 \%$ and $6 \%$ higher than for 3.9 and $5.3 \mathrm{~nm}$ cap thickness, respectively. The deviations are significantly larger than the measurement error of $\approx 2-3 \%$. This distinct optimum is the minimum GaAs coverage over the QDs prior to heating, in order to prevent indium segregation towards the surface, or the dissolution of not completely covered QDs during heat up [Nun05]. Larger thicknesses than this required minimum of the LT-GaAs layer increases the number of non-radiative defects (most likely 


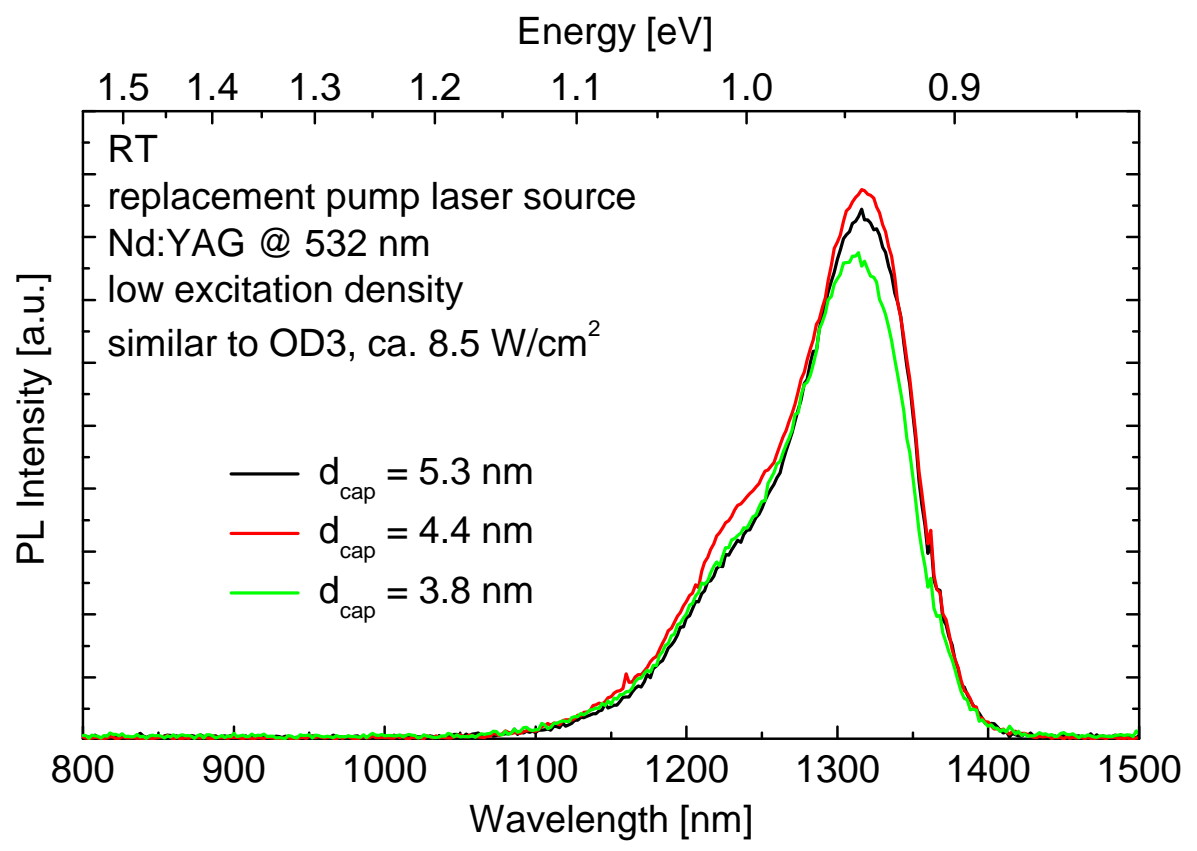

Figure 5.18: PL measurements of samples containing a single $I n G a A s$ QD layer and a SRL with varied LT-GaAs cap dimensions are shown. A LT-GaAs cap thickness of $4.4 \mathrm{~nm}$ shows a distinct maximum of integral luminescence intensity.

vacancies near the QDs). As shown in 4.1.2 the V/III ratio plays an important role during spacer growth as well due to its influence on group III vacancy formation.

All three low temperature layers of the QD growth process were optimized regarding these two parameters in statistically planned experiments using a design-of-experiment software package ${ }^{1}$. This is useful due to the cross-influence of the varied parameters resulting in a complex impact on InGaAs QD composition, layer thickness, and material quality. Indeed, the analysis of a set of 32 samples yielded an optimized set of parameters reproducibly giving the highest PL intensity among these samples. These optimum parameters resulted in complete suppression of wavelength blue shift upon annealing of a five-fold QD stack with ground state luminescence at $1324 \mathrm{~nm}$. Figure 5.19 shows low excitation density (OD3) PL as grown and annealed from this optimized sample, and for a reference a non-optimized sample (dashed). This evidences that complete blue shift suppression is possible for QDs emitting at more than $1.3 \mu \mathrm{m}$ by low excitation PL optimization to prevent defect creation in the vicinity of QD layers.

Experiments show that the V/III ratio has an especially important impact on vacancy formation during growth at low temperature. The question arises whether the effect of V/III related vacancy formation occurs also during further overgrowth at higher temperature

${ }^{1}$ Design-Expert ${ }^{\circledR}$ version 7.1.3 by Stat-Ease, Inc. 


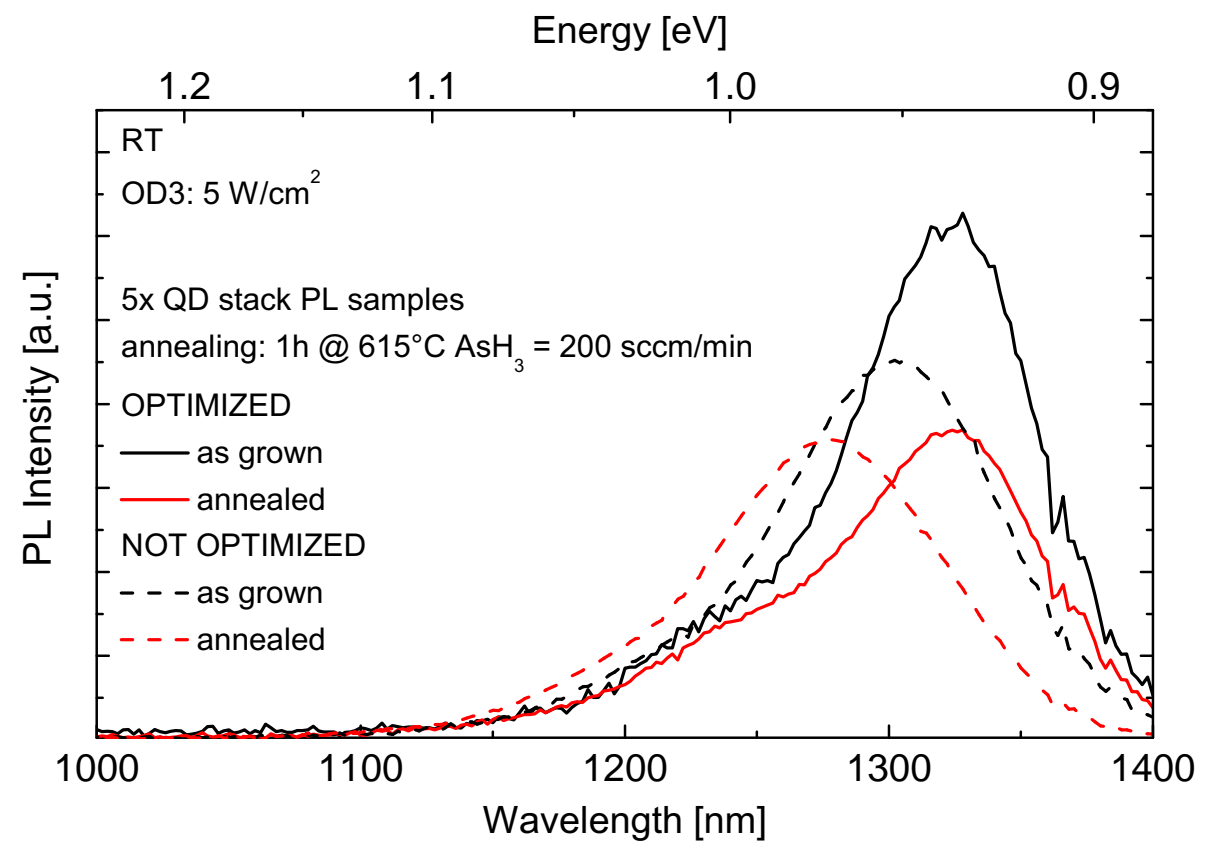

Figure 5.19: PL measurements of samples containing five-fold In GaAs QD stacks as grown and annealed for $1 \mathrm{~h}$ at $615^{\circ} \mathrm{C}$. Luminescence intensity of both samples was reduced to the same level upon annealing. solid lines: Sample with optimized parameters for highest OD3 luminescence shows no wavelength blue shift. dashed lines: Reference sample of Figure 5.16 not optimized for highest OD3 luminescence shows inferior thermal stability and exhibits a $\approx 30 \mathrm{~nm}$ blue shift upon annealing.

of a QD stack already capped by at least a full $45 \mathrm{~nm} \mathrm{GaAs}$ spacer layer. To investigate the influence of the V/III ratio during cladding growth, pieces of the same sample as in 5.1 are annealed at $700^{\circ} \mathrm{C}$ with TBAs flows differing by a factor of four. Figure 5.20 shows that the lower TBAs flow significantly reduces the emission blue shift at high temperatures, from $34 \mathrm{~nm}$ to only $6 \mathrm{~nm}$. The observed effect is attributed to the formation of surface vacancies caused by excess arsenic on the surface supporting indium segregation by vacancy diffusion. This finding supports the model that the indium interdiffusion is driven by group III vacancies, and that arsenic supply can control the formation of vacancies at the surface or within the crystal during growth. Additionally, this result demonstrates the long reach of this effect which, in this case, affects QDs at a distance of $150 \mathrm{~nm}$ or more.

In conclusion, optimal integration of QDs and SRL into the surrounding GaAs matrix crystal is the crucial point in suppressing defect formation. Additionally, spacer growth needs to achieve a perfectly smooth, strain-free surface after every QD layer to enable optimal QD growth conditions for subsequent layers. For all subsequent overgrowth layers the long range of vacancy driven interdiffusion needs to be taken into account by choosing 


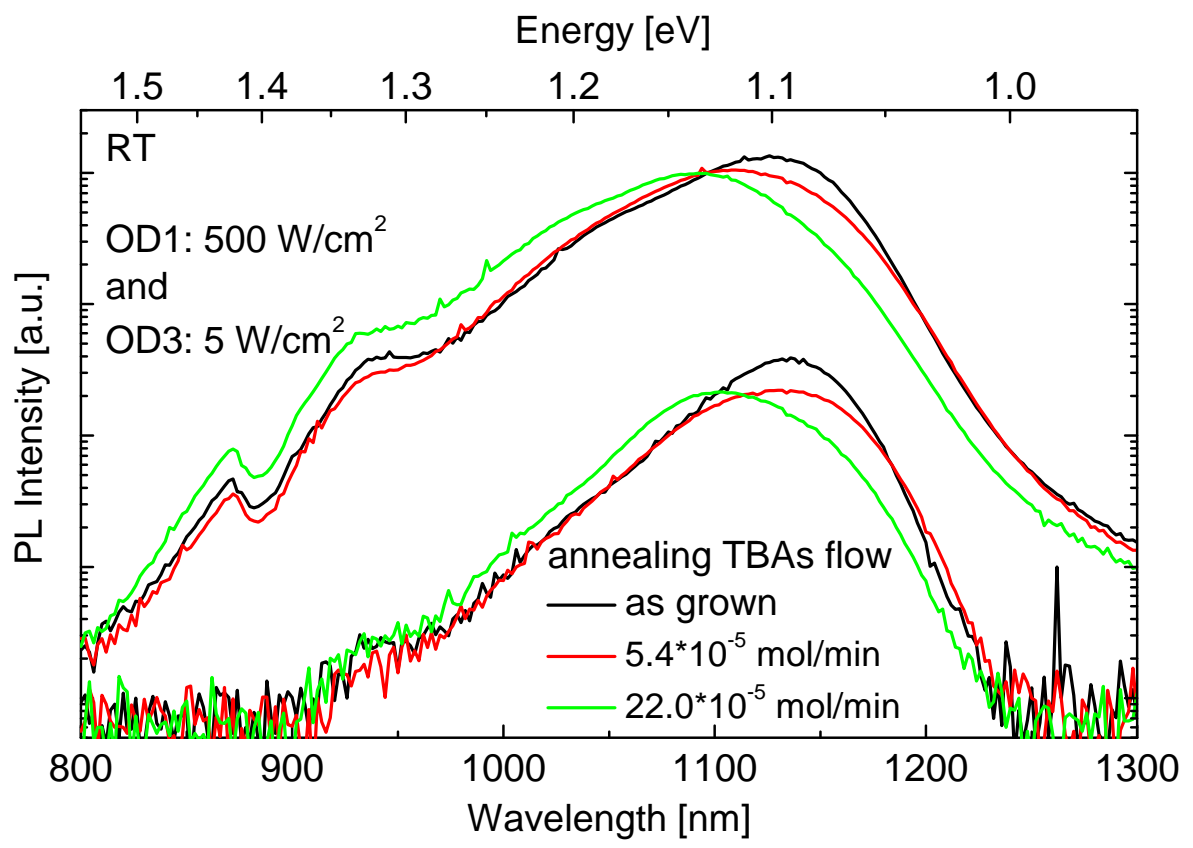

Figure 5.20: Identical single PL test sample with three-fold $I n_{0.75} G a_{0.25} A s$ QD stack as in 5.1 is annealed with varied TBAs stabilization flows and compared to the grown data. Annealing conditions in the reactor are: 30 min duration, $\mathrm{H}_{2}$ carrier gas, $15 \mathrm{l} / \mathrm{min}$ total flow, $100 \mathrm{mbar}$ pressure at $700^{\circ} \mathrm{C}$ reactor temperature. The TBAs reduction by a factor of 4 to $5.410^{-5} \mathrm{~mol} / \mathrm{min}$ significantly lowers the blue shift caused by the annealing process compared to high TBAs flow.

appropriate growth temperatures and V/III ratios. These results on blue shift suppression are published in $[$ Str08a].

\subsubsection{Substrate size effects}

To economize for expensive GaAs substrates, optimization of QD parameters and other calibration runs are often carried out on quarter wafers or even smaller substrate pieces. In our case 2" wafers are split by hand with a scalpel into equal quarter pieces and used on a custom-built quarter wafer susceptor. This procedure is without problems up to the point of final device growth runs, which are usually carried out on full 2" wafers. The change of sample size causes temperature deviations on the wafer surface for nominally identical process temperatures by altering the temperature offset between susceptor thermocouples and wafer surface. A direct precise measurement of this offset change by the available pyrometric in-situ sensor lacks the required accuracy for QD growth processes. This offset change due to different wafer sizes is triggered by a change in the overall emissivity of the carbon plate and wafer system. According to Kirchhoff's law of thermal radiation, emissivity of a body (or surface) equals its absorptivity in thermal equilibrium. By using a 


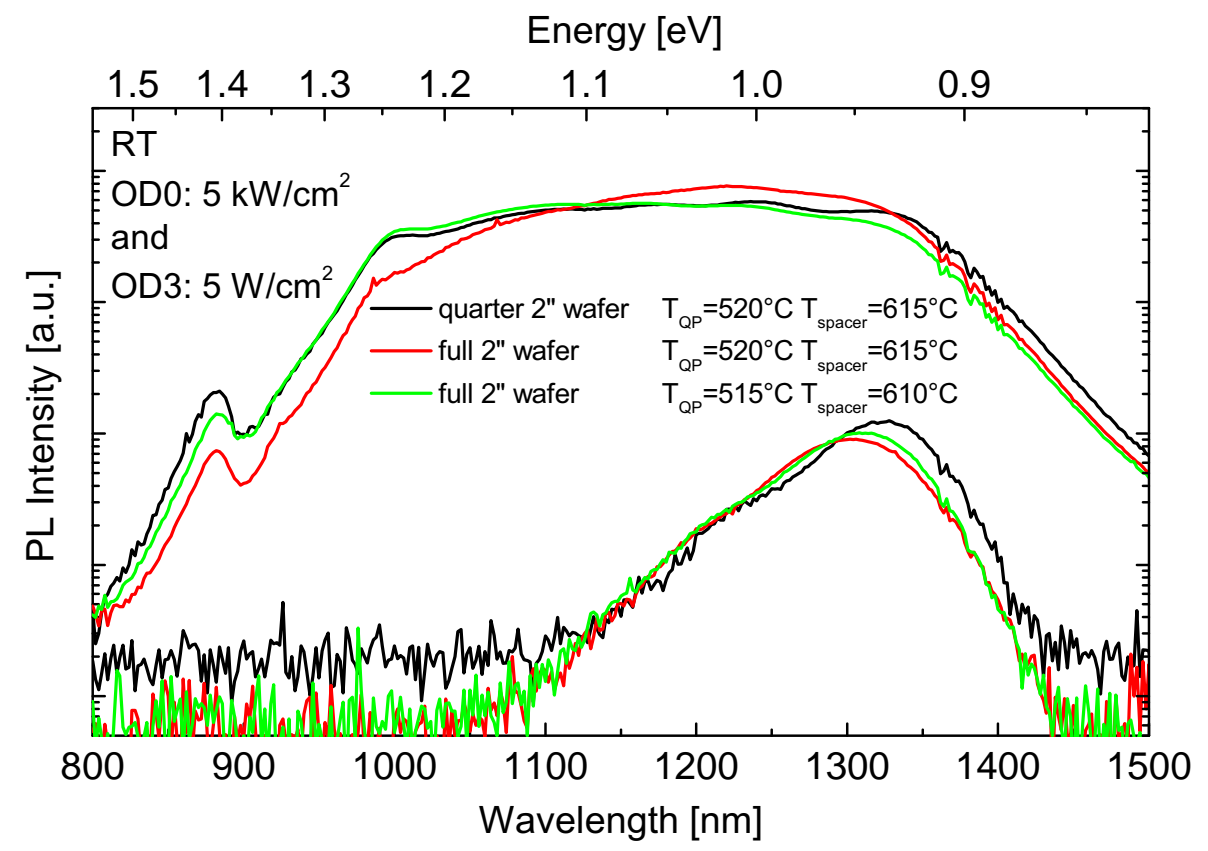

Figure 5.21: PL measurements from samples containing a five-fold QD stack grown on different substrate sizes are shown. The process change from a 1/4 wafer to a full 2" wafer causes a 22 $\mathrm{nm}$ blue shift and a decreased OD3 luminescence. A third sample grown with adapted QD and spacer growth temperatures to compensate for the altered temperature offset shows a slight OD3 luminescence improvement and reduces the blue shift to $14 \mathrm{~nm}$.

larger wafer size a part of the highly absorbing susceptor surface is replaced by the highly reflective mono crystalline wafer surface. In consequence, the reduced overall emissivity reduces the temperature offset. This effect was measured with aluminum-silicon eutectic wafers of different sizes to $\approx 5^{\circ} \mathrm{C}$. Thus, the real wafer surface temperature for a full 2 " wafer is increased compared to a quarter wafer for the same set process temperature. This is an important finding for all highly optimized nanostructure growth processes as QD formation are particularly well known to be highly temperature sensitive [Hei96].

The influence of wafer size change on optical properties of a highly optimized five-fold $1.3 \mu \mathrm{m}$ QD stack is shown in Figure 5.21. The sample grown on a full wafer is $22 \mathrm{~nm}$ blue shifted due to the altered growth conditions as compared to the reference, and shows decreased OD3 luminescence. This decrease is attributed to sub-optimal growth conditions causing increased formation of non-radiative defects (most likely vacancies) near or within the QD layer. The measurement at high OD0 excitation shows stronger but blue-shifted luminescence due to the saturation of non-radiative defects, and most likely a higher QD density. An additional sample with modified QD and spacer growth temperatures according to the eutectic measurement results was grown to compensate for the temperature change during growth. The compensated sample did show a blue shift reduction to $14 \mathrm{~nm}$ and a 


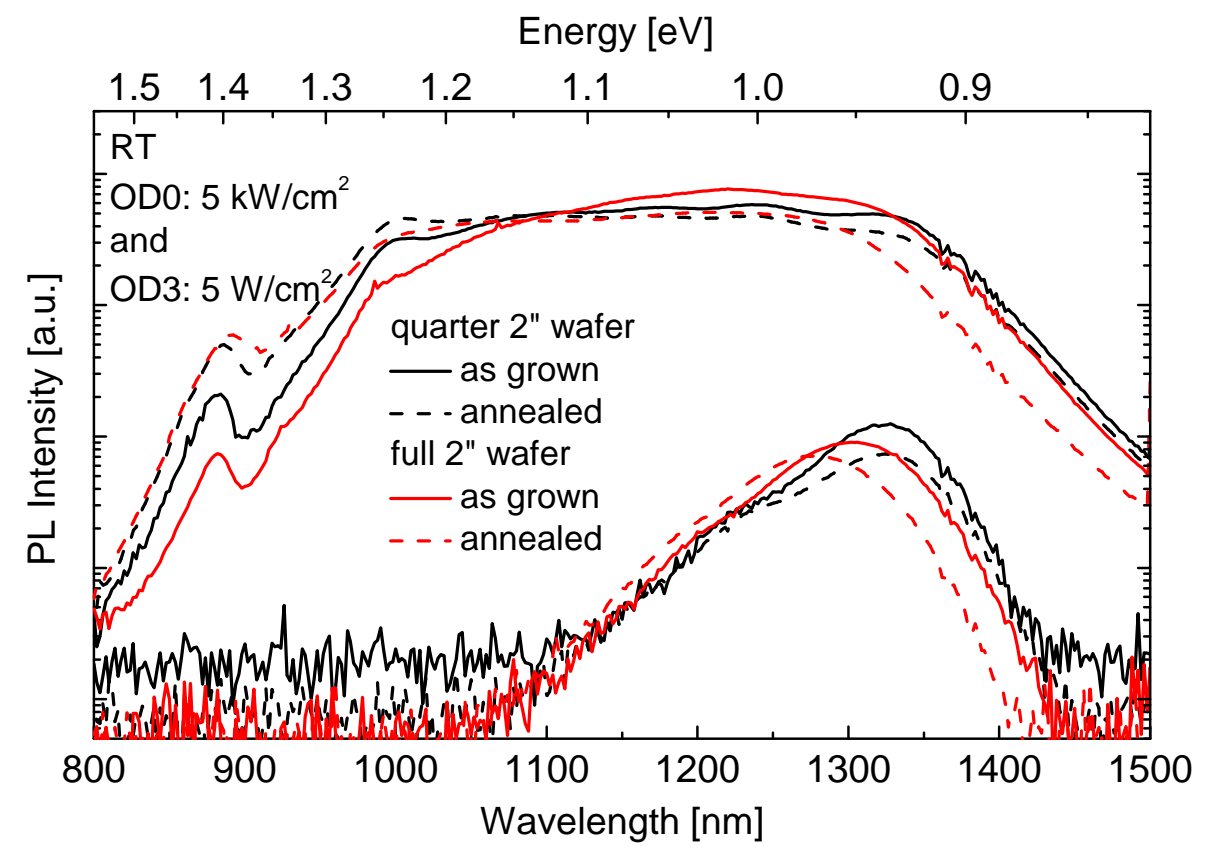

Figure 5.22: PL measurements from samples containing a five-fold QD stack grown on different substrate sizes are shown. Both samples grown on either a $1 / 4$ wafer or a full 2" wafer are measured as grown and after $1 \mathrm{~h}$ annealing at $615^{\circ} \mathrm{C}$. Parameters are optimized for the $1 / 4$ wafer process and show perfect spectral annealing stability. The full wafer sample is $24 \mathrm{~nm}$ blue shifted upon annealing in addition to the $22 \mathrm{~nm}$ blue shift of the grown sample.

slight luminescence improvement at OD3 excitation. OD0 measurement however shows the same characteristics as the initial quarter wafer sample but with added blue shift.

This only partial compensation by adapted growth temperature is attributed to a change of available decomposed precursor adatoms on the surface. Precursor decomposition rates rely not only on thermal decomposition, but also on catalytic surface processes. These are most likely influenced by an altered ratio of available wafer to susceptor surface area due to differing surface conditions in terms of roughness and available crystalline facets. In consequence, growth rates and layer thicknesses can be altered. This substrate size becomes especially visible for highly optimized QD structures in the long wavelength regime, which are extremely sensitive to any change. Thus, thermal stability upon annealing and overgrowth was significantly decreased as demonstrated in Figure 5.22.

In conclusion, no simple growth parameter adaptation is possible to compensate for a change in wafer size. To overcome this issue a QD optimization process needs to be performed on the same substrate size as for the final device growth runs, otherwise the additional effort in time and cost for further process adaptation to the final wafer size needs to be accepted. 

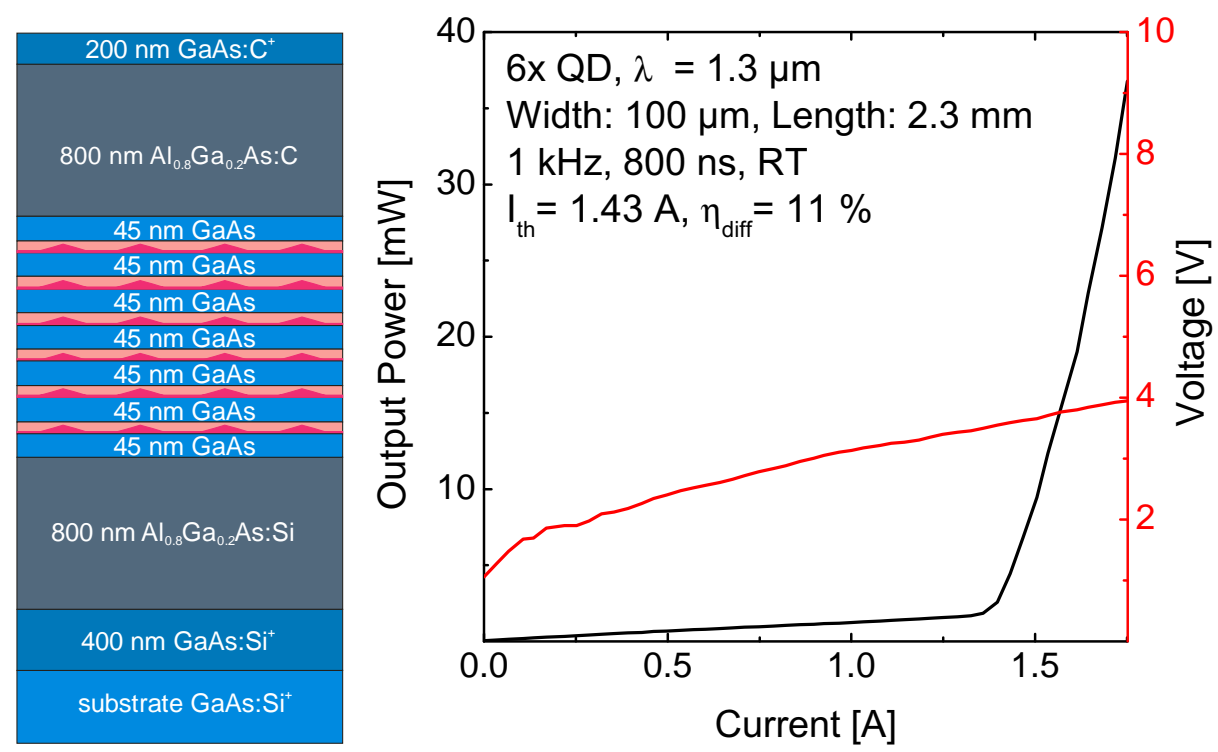

Figure 5.23: left: Schematic of the grown $1.3 \mu \mathrm{m}$ laser structure employing $A l_{0.8} G a_{0.2} \mathrm{As}$ claddings with $\mathrm{Si}$ and $\mathrm{C}$ doping and a highly optimized six-fold QD stack active region to suppress blue shift upon overgrowth. right: L-I-V curves under pulsed injection at room temperature.

\subsection{3 $1.3 \mu \mathrm{m}$ laser design}

Realization of the first working In $G a A s$-QD $1.3 \mu \mathrm{m}$ edge-emitter without using any additional materials (e. g. antimony as a surfactant) proves the successful suppression of the emission blue shift for complete lasers and the feasibility of such devices by MOVPE. The $1.3 \mu \mathrm{m}$ edge-emitter design is enabled through the strategic optimization of process parameters for successful overgrowth of stacked QD structures without unintentional blue shift (cf. 5.2.1). Additionally, the insight gained on substrate size effects depicts the necessity for retaining a chosen wafer size for final devices (cf. 5.2.2). Consequently, final device structures are grown on the same quarter wafer-sized substrates as were used for process parameter tuning. Processing and mounting employs the same broad waveguide Schnellprozess as the previously depicted lasers without any high reflection coating for the facets or individual mounting of the lasers to advanced heat spreaders.

A schematic of the epitaxial design is shown in Figure 5.23. Layer structure and epitaxial process are based on the AlGaAs : Si/C laser as described in 5.1. Most significantly, the complete growth process for all layers was transferred from a TBAs-based to an $\mathrm{AsH}_{3}$-based process, except for the low temperature QD growth at $520^{\circ} \mathrm{C}$. Bottom $A l G a A s$ : Si cladding thickness was reduced to $800 \mathrm{~nm}$, QD layer period was about $5 \mathrm{~nm}$ shorter than in the previous design, and a sixth QD layer was added to the active 


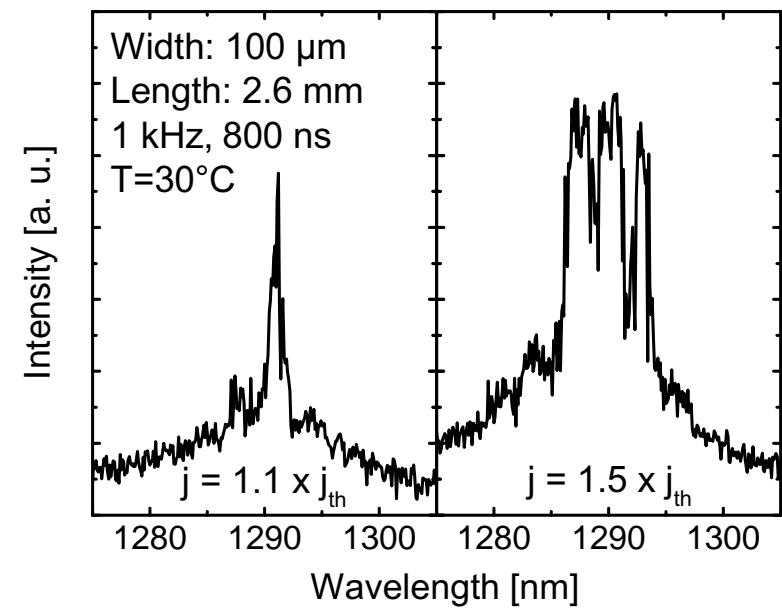

Figure 5.24: Laser emission spectra around $1.3 \mu \mathrm{m}$ :

left - close to lasing threshold;

right - at 50\% above the laser threshold current density.

stack. Relatively low V/III ratios as compared to conventional growth parameters were used throughout the structure. Use of $\mathrm{AsH}_{3}$ renders direct comparisons to the previous TBAs-based process difficult. This applies especially to spacer $\left(\mathrm{AsH}_{3} / \mathrm{III}=91\right)$ and top cladding $\left(\mathrm{AsH}_{3} / \mathrm{III}=247\right)$ growth at $615^{\circ} \mathrm{C}$. Both claddings are uniformly doped with either $\mathrm{SiH}_{4}$ or $\mathrm{CBr}_{4}$.

Lasing is achieved at a threshold of $j_{t h} \approx 600 \mathrm{~A} / \mathrm{cm}^{-2}$. Emission spectra are shown in Figure 5.24 demonstrating laser emission up to $1293 \mathrm{~nm}$ at $30^{\circ} \mathrm{C}$ for a $2.6 \mathrm{~mm}$ device. The data for a variety of differently sized devices are given in Figures 5.25 and 5.26 Transparency current density was calculated to $j_{t r}=284 \pm 32 \mathrm{~A} / \mathrm{cm}^{2}$ based on data from devices with three different lengths. Precise meaningful data on internal efficiency and losses is not calculable due to the large variations in $1 / \eta_{\text {diff }}$ compared to threshold values. A rough approximation based solely on cavity lengths of $1.6 \mathrm{~mm}$ and $2.3 \mathrm{~mm}$ is $\eta_{\text {int }} \geq 25 \%$. This wide range of device parameters indicates that an increased number

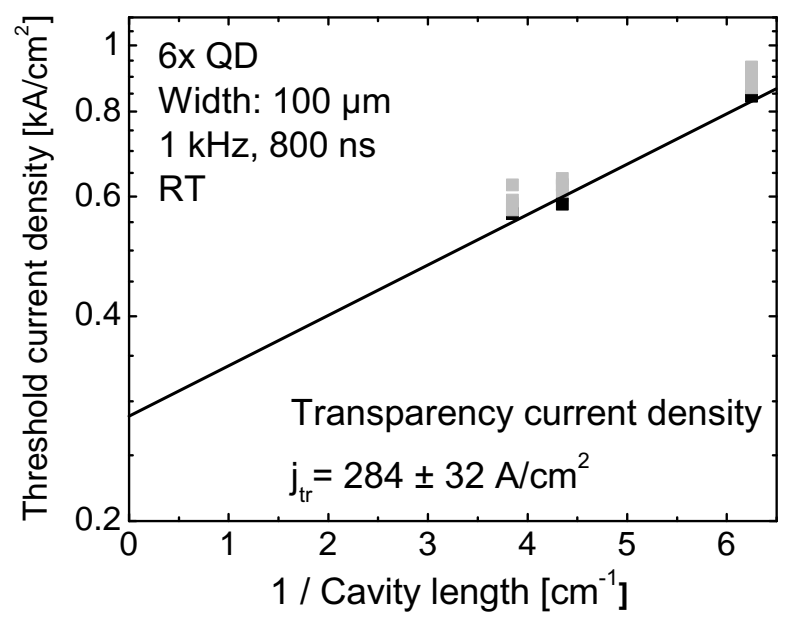

Figure 5.25: The transparency current density is estimated based on three data points to an elevated value of $j_{t r}=284 \mathrm{~A} / \mathrm{cm}^{2}$ compared to previous devices. 
Figure 5.26: A meaningful fit to calculate internal efficiency $\eta_{\text {int }}$ and internal losses $\alpha_{i}$ is not possible due to the strong variation in measured results. A rough approximation based solely on the best devices with cavity lengths of $1.6 \mathrm{~mm}$ and $2.3 \mathrm{~mm}$ is $\eta_{\text {int }} \geq 25 \%$.

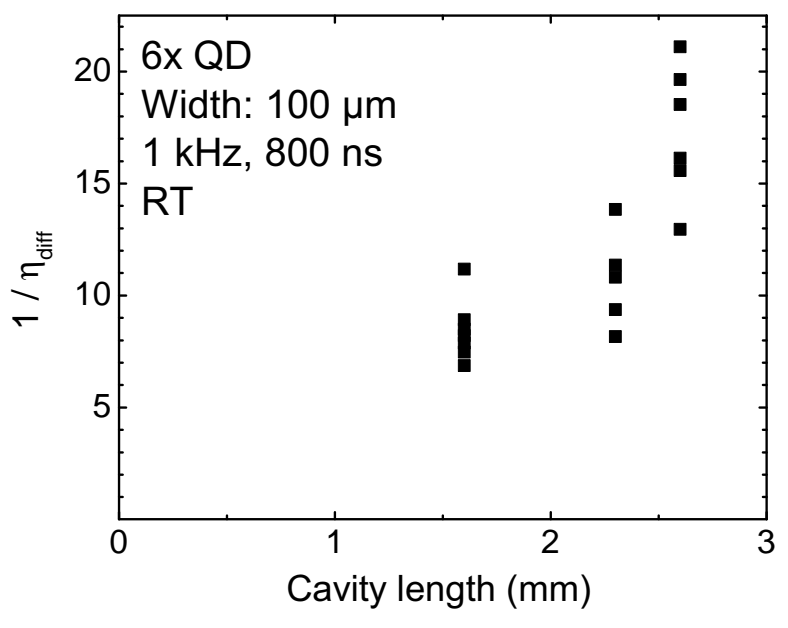

of defects within the structure were generating losses. In consequence, threshold values were elevated and especially long devices showed inferior performance, whereas very short devices did not achieve lasing.

Despite the elevated intrinsic losses this device enabled, for the first time, successful demonstration of MOVPE grown QD laser emission at $1.3 \mu \mathrm{m}$ wavelength without any additional sophisticated techniques, like antimony irradiation. This success is based on the optimized MOVPE growth process for stacked QDs enabling complete blue shift suppression. 


\subsection{Summary and Outlook}

In this work on QD based edge-emitting lasers, individual parameters of the QD deposition and QD capping process have been systematically studied to improve QD properties and thermal stability. Variable excitation density PL measurements were strategically used as a fast characterization tool to individually tune QD properties and to optimize the growth process for thermal stability. Optimized QD growth processes resulted in significant improvement of edge-emitter properties and longer wavelength emission up to $1.3 \mu \mathrm{m}$. The understanding and adaptation of individual growth parameters allowed for a complete suppression of unintentional QD blue shift upon overgrowth.

Key QD growth parameters identified during this research are:

- The long range influence of the V/III ratio on indium interdiffusion out of the QD layer is based on increased vacancy diffusion.

- Capping layers V/III ratio has a direct strong influence on final QD parameters and QD annealing stability.

- Final thermal stability of buried QDs and QD stacking efficiency are defined by the defect density in the vicinity of the QD layer. Through defect-free integration into the surrounding GaAs matrix material by an optimal QD capping process, emission blue shift can be completely suppressed.

Based on the improved QD growth processes broad waveguide devices were realized with ultra-low thresholds. Different dopant materials and doping schemes were investigated and employed within complete laser devices. Improvements of QD overgrowth stability and stacking efficiency enabled the realization of the first $1.3 \mu \mathrm{m}$ QD edge-emitter grown by MOVPE without any additional mediating materials.

An aluminum-free edge-emitter design was developed with lattice matched InGaP claddings demonstrating excellent laser performance. Based on this design, a taper process for QD-based optical amplifiers was developed in cooperation with partners within the OptiDot project.

Future work should focus on high density QD layers with a reduced homogeneous broadening to enable further improved laser performance. Additionally, waveguide design improvements are crucial to reduce internal losses. Lattice matched InGaP should be further investigated as an $A l G a A s$ alternative.

Completely novel laser designs such as photonic band crystal (PBC) or distributed feedback (DFB) lasers could benefit from the intrinsically broader gain spectrum of SK-QDs. PBC lasers are a promising approach toward generating efficient high-power 
and high-brightness laser sources, employing an edge-emitting design with an extended waveguide forming a one-dimensional photonic crystal [Ket10]. DFB lasers employ an etched periodic structure, which is subsequently overgrown to achieve high performance single mode operation. Thus DFB lasers require an oxide-free surface prior to the second epitaxy which can be realized by In $\mathrm{GaP}$ claddings. The broad emission spectra of QD active structures in DFB lasers enables temperature stable long wavelength operation, and is likely to be beneficial for high speed mode locking [Tak11]. 


\section{Chapter 6}

\section{High-power vertical external-cavity surface-emitting lasers}

Optically pumped VECSELs, also known as semiconductor disk lasers, are an extremely flexible design with a unique set of advantages [Kuz99, Has03, Tro04]:

- High output powers up to tens of watts in CW mode.

- Outstanding beam quality with true $\mathrm{TEM}_{00}$ emission.

- Intra-cavity access enables efficient frequency doubling.

These features are achieved by building an extended cavity outside of a semiconductor gain-chip. Thus, opposite to all other laser concepts in this work, the VECSEL design is not monolithic and requires multiple separate elements for operation. Usually separate mirrors define the outer cavity part while the gain-chip defines the other semiconductor end of the cavity, and can essentially be regarded as a VCSEL without top DBR. In fact, a VCSEL can be converted to a VECSEL by removing the top DBR [Lot05]. A separate diode laser focused on the gain-chip surface typically generates optical pumping of the gain-chip. In consequence, this complex design necessitates careful alignment of all elements: pump laser, gain-chip, and external cavity mirrors. Employed VECSEL setups are briefly presented in this Chapter while the focus is on the epitaxial fabrication of the demanding semiconductor gain-chip structures based on SML and QD active media.

For this versatile VECSEL concept many different design implementations exist. The minimal setup to realize a VECSEL cavity includes at least one semiconductor gainchip and one external mirror, if the latter serves simultaneously as output coupler (OC). This basic cavity design can be altered to include multiple gain-chips, mirrors, and any other optically-active elements, and can extend to a total cavity length of more than 1 $\mathrm{m}$ [Jac07]. Support for single mode operation and emission wavelength tuning can be achieved using an intra cavity etalon [Tou07, Fan07a]. The introduction of a semiconductor saturable absorber mirror within the VECSEL cavity can be used for high speed passive mode-locking and the generation of short pulses [Asc05, Rut06, Klo08, Ino06, Lag07]. Very efficient second harmonic generation (SHG), up to $27 \%$ from pump power to second harmonic output, can be realized by the introduction of a nonlinear crystal into the VECSEL 
cavity [Lee06, Här07]. SHG in cavities can become highly efficient as a result of the high optical field intensity within the resonator as compared to the external laser beam, and can attain conversion efficiencies of more than $80 \%$ [Ou92, Pas94]. In consequence, the flexibility of the VECSEL design enables the use of $\mathrm{GaAs}$ based nanostructures for high power, $\mathrm{CW}$ applications in the visible wavelength range [Cal09].

VECSEL gain-chips are usually optically pumped for high power operation. This enables homogenous pumping up to very large diameters of hundreds of microns and pumping of large active stacks with tens of active layers to maximize optical gain [Bey05. Kim06, Bey07]. These active layers within the gain-chip are usually positioned as resonant periodic gain structure (RPG) to maximize the overlap with the optical field intensity. An integrated DBR on one side and a window layer on the other side typically surround this RPG. The window layer needs to be transparent to the generated light, and functions as a barrier to prevent surface recombination of free charge carriers. Power scaling is achieved by increasing the pump spot size, while total pump power is finally limited by the finite heat flow in the semiconductor gain-chip [Che10]. Often transparent intra cavity heat spreaders bonded to the surface and/or substrate removal techniques are employed to improve gain-chip heat-removal characteristics. Multi gain-chip designs are possible to further increase optical output power, employing multiple separately pumped beam spots [Fan06].

All VECSEL device development and growth in this work was carried out as part of the European NATAL project. Target wavelengths of the NATAL project cover a wide spectral range, with target wavelengths of $940 \mathrm{~nm}, 1040 \mathrm{~nm}$, and $1220 \mathrm{~nm}$. These wavelengths were chosen to enable GaAs-based devices for red, green, and blue lasing by intra-cavity SHG for wide color gamut projection applications. In parallel to conventional designs based on QW gain media that were developed by NATAL project partners, VECSEL designs with novel gain concepts targeting the same emission wavelengths were developed within this work at TU-Berlin. Conventional QW based VECSELs suffer from poor thermal stability due to the narrow QW gain spectra, shifting out of resonance with temperature. In consequence, the pump threshold is increased and differential efficiency is decreased for higher temperatures [Kuz99, Tro04]. QDs are proposed to be able to overcome this inherent limitation by using the much broader gain spectrum compared to QW emission. Another advantage is the wide spectral tuning range of QDs. Additionally, SML structures were introduced for the first time as VECSEL active media in order to benefit from the higher peak gain compared to SK QDs.

In this work MOVPE processes for active layer growth, and subsequently for VECSEL fabrication, were developed for all three target wavelengths. Multiple VECSEL designs had to be developed to minimize optical losses. Structures with up to $\approx 8 \mu \mathrm{m}$ total thickness needed to be grown while maintaining excellent crystal quality, lateral homogeneity, and 
smooth surfaces to enable lasing operation. However the main challenge of this project was the growth of thick active cavities ( $1-2 \mu \mathrm{m})$ including stacks of up to 30 strain-inducing active layers, while maintaining high optical quality and stable QD properties. Such large stacks are required to achieve sufficient modal gain, especially for QDs with a reduced peak gain compared to QWs.

\subsection{Device mounting and characterization}

Device mounting and characterization of the presented devices was carried out at $\mathrm{ORC}^{1}$ at Tampere University of Technology, Finland as part of a cooperation within the European NATAL project. For initial on-site characterization, a compact VECSEL setup was developed at TU-Berlin as part of a diploma thesis [Poh08a].

As a vertical emitting, optically pumped device no special processing of the grown VECSEL gain-chip wafer was necessary. Square pieces with dimensions of a few millimeters were easily cleaved and mounted onto a temperature-controlled copper heat sink. Efficient heat removal from the wafer surface was realized by transparent intra-cavity heat spreaders liquid-capillary bonded to the gain-chip surface.

\subsubsection{ORC VECSEL setup}

A schematic drawing of the employed setup is given in Figure 6.1. No thinning of the gain-chip wafer or other processing was carried out. A cleaved VECSEL gain-chip piece of $2.5 \times 2.5 \mathrm{~mm}^{2}$ was liquid-capillary bonded to a $250-300 \mu \mathrm{m}$ thick, natural type IIa diamond heat spreader, and both were clamped together to the water-cooled copper heat sink. The temperature of the heat sink was set to $\mathrm{T}=15^{\circ} \mathrm{C}$ unless otherwise mentioned. Optical pumping up to $20 \mathrm{~W}$ was realized by an $808 \mathrm{~nm}$ fiber-coupled diode laser at an incident angle of $35^{\circ}$. Pump laser light was focused to a pump spot with a diameter of $d$ $=180 \mu \mathrm{m}$ and the laser mode size was matched to the pump size. The V-shaped cavity separates the concave focusing mirror with a radius of curvature (roc) of $200 \mathrm{~mm}$ from the OC. Thus the OC can be easily changed without modifying the alignment. All VECSEL results in this work, using this setup, were measured by the colleagues J. Rautiainen, M. Guina, and O.G. Okhotnikov at ORC, Finland.

\subsubsection{TU-Berlin VECSEL setup}

As part of the diploma work of J. Pohl, a compact VECSEL setup was developed at TU-Berlin in parallel to the NATAL project and to the development of VECSEL structures.

\footnotetext{
${ }^{1}$ Optoelectronics Research Centre, Tampere University of Technology, Korkeakoulunkatu 3, Tampere 33720, Finland
} 


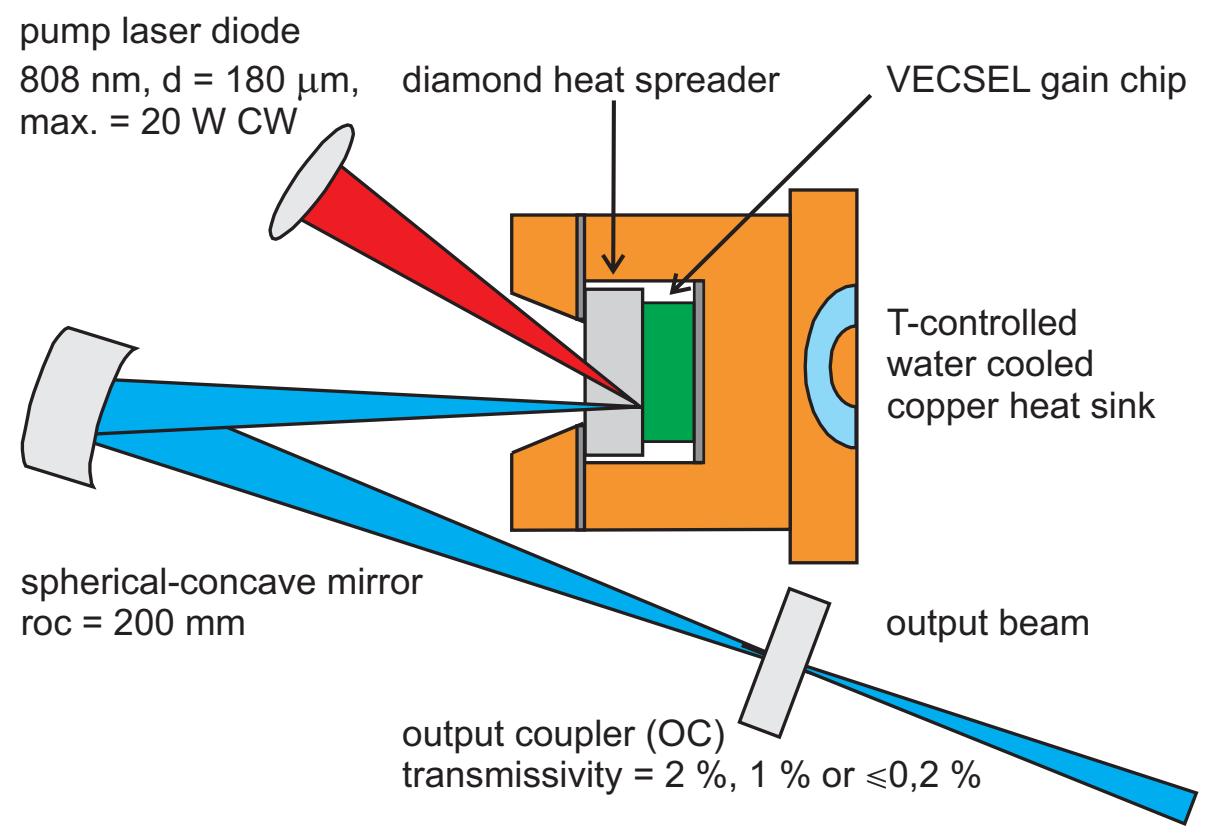

Figure 6.1: Schematic drawing of the employed VECSEL setup at ORC, Finland. The Setup employs a V-shaped cavity design with separated OC and focusing mirror. All VECSEL device results presented were achieved with this setup unless otherwise mentioned. This setup and the VECSEL development are part of the European NATAL project.

Figure 6.2 shows a schematic of this linear setup design. The base of the copper heat sink is constantly water cooled. A Peltier element separating this base from the sample holder allows for accurate temperature control of the gain-chip. Surface heat-spreader and gain-chip were clamped to the copper sample holder. The second reflector of the linear cavity is formed by a spherical-concave mirror with roc $=50 \mathrm{~mm}$, serving simultaneously as OC. Optically pumping up to $4.5 \mathrm{~W}$ was performed by an $808 \mathrm{~nm}$ fiber-coupled diode laser at variable incident angles. The optical fiber core with a diameter $\mathrm{d}=50 \mu \mathrm{m}$ was directly imaged at the sample surface by two lenses with focal lengths of $\mathrm{f}=40 \mathrm{~mm}$. Minimum beam spot diameter was measured to $d=58 \mu \mathrm{m}$. This pump spot diameter is relatively small compared to the above cited literature which uses diameters from $\mathrm{d} \approx 100$ $\mu \mathrm{m}$ to $\mathrm{d} \approx 500 \mu \mathrm{m}$. In consequence, high optical pump power densities up to $\approx 170 \mathrm{~kW}$ were achievable, but alignment proved to be more difficult and boundary effects of the pumped area were more relevant due to the higher boundary/pump-area ratio.

For semiconductor gain-chip heat removal, transparent $\mathrm{SiC}$ or diamond intra-cavity heat spreaders and sample thinning to $\approx 100 \mu \mathrm{m}$ were investigated. Tests with a SiC heat spreader liquid-capillary bonded to the gain-chip did not achieve lasing, despite its good thermal conductivity of $\kappa_{S i C}=490 \frac{\mathrm{W}}{\mathrm{mK}}$. This is attributed to a high background doping of the employed $\mathrm{SiC}$ sample causing increased losses. Successful lasing operation was 


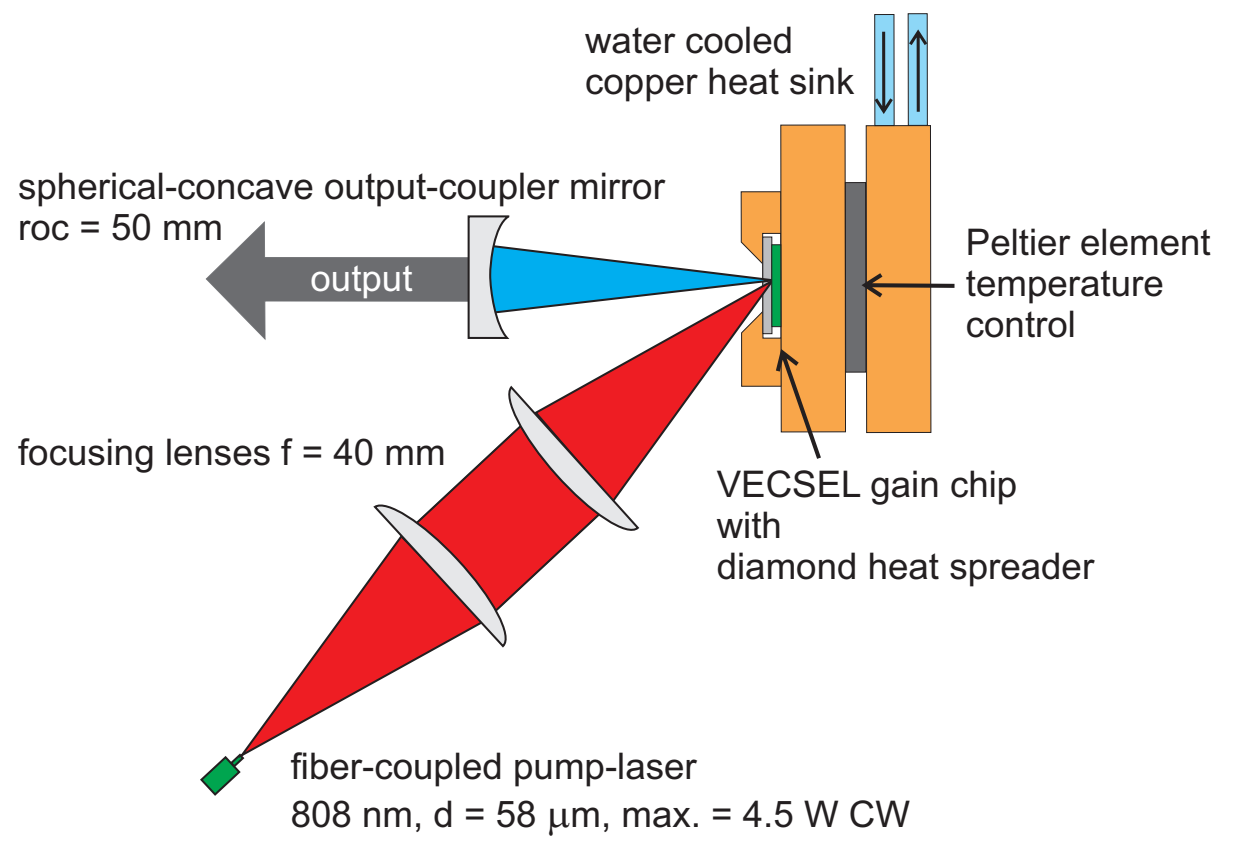

Figure 6.2: Schematic of the VECSEL setup realized by J. Pohl et al. at TU-Berlin in 2008 . Measured pump beam focus diameter is $d=58 \mu \mathrm{m}$. The compact design employs a combined focusing mirror and OC element. Further details are given in the corresponding diploma thesis [Poh08a].

achieved with a diamond heat spreader bonded in the same way to the wafer. Diamond offers outstanding characteristics for heat removal directly from the wafer surface with its inherently excellent thermal conductivity of $\kappa_{\text {diamond }}=1600 \frac{\mathrm{W}}{\mathrm{mK}}$ and good transparency [Lee06, Här06]. Further details on the setup characteristics and alignment methods can be found in the corresponding diploma thesis J. Pohl [Poh08a]. 


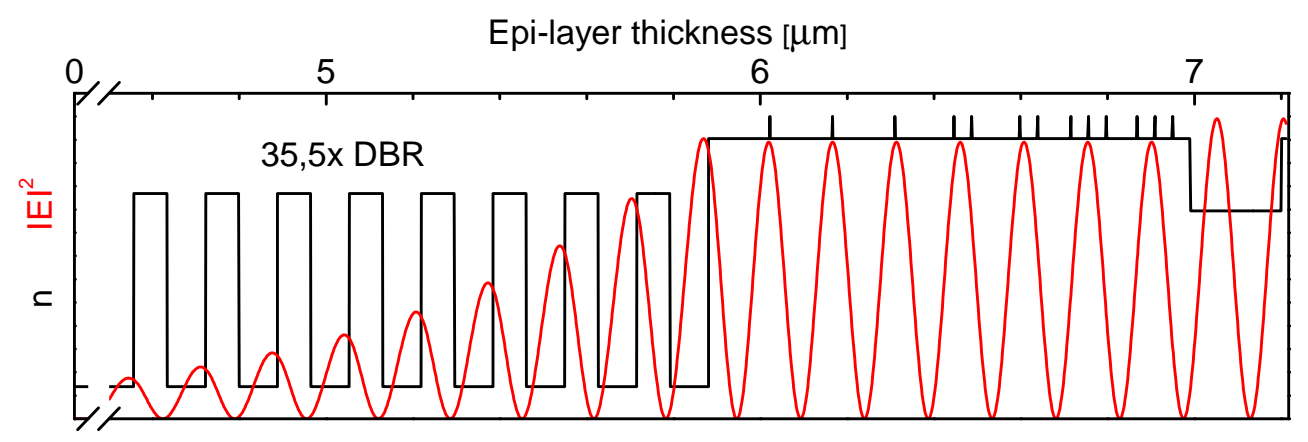

Figure 6.3: Transfer matrix simulations of the refractive index and the optical standing wave pattern of the nonlinear VECSEL design show the overlap of the antinodes with the grouped active layers.

\subsection{Initial nonlinear gain structure design}

The nonlinear design comprises a DBR with 35.5 pairs of $A l_{0.98} G a_{0.02} A s / A l_{0.2} G a_{0.8} A s$ $\lambda / 4$ layers, yielding a nominal bottom reflectivity of $99.92 \%$ and $90 \mathrm{~nm}$ spectral width of the stop band. On top of the DBR, a RPG sub-cavity is grown comprising 10 to 13 active layers within a $G a A s$ matrix. The structure terminates with an $A l_{0.3} G a_{0.7} A s \frac{3}{4} \lambda$ window layer with a $10 \mathrm{~nm}$ GaAs oxidation protection cap. Grouping active layers at the antinodes of the optical standing field maximizes the overlap, and thus the confinement factor. To account for the exponentially decreasing pump light intensity, and to ensure population inversion for all active layers, the number of active layers per antinode decreased with the distance from the window layer. A group of 3 active layers was placed at the two top-most antinodes followed by 2 active layers at the $3^{r d}$ and $4^{\text {th }}$ antinodes, and a single active layer for all following standing wave maxima (cf. Figure 6.3). Growth of the whole design was carried out only by alternative precursors as a single lengthy epitaxial growth run. All layers were nominally undoped, but $A l G a A s$ notably exhibits a non negligible background doping level as detailed in Section 4.2 .

\subsubsection{Active media}

Initial investigations were focused on nanostructures producing a maximum gain per active layer. Two different approaches were chosen for active media of initial devices:

- SML structures as detailed in Section 2.2.3 exhibit a similar peak gain compared to QW structures. Compared to SK-QDs the FWHM is significantly smaller, entailing precise spectral alignment with the cavity resonance. Employed SML structures are tuned for VECSEL operation to use ground state (GS) emission. 
- Excited state transitions of QD layers grown in the SK growth mode, employing the highly optimized process as detailed in Chapter 4 were used. Thus, thermal stability for overgrowth and highest modal gain per layer was assured. SK-QD emission was tuned to longer wavelengths as compared to the VECSEL cavity resonance in order to benefit from increased gain per QD layer by using excited state (ES) transitions.

Ground state emission of SML structures was tuned to $940 \mathrm{~nm}$ and $1040 \mathrm{~nm}$ peak emission using measurements of PL test structures. Controlled wavelength shift of the SML structures was achieved by altering the GaAs spacer thickness, InAs per SML deposition, and/or number of periods within a single SML structure. As SML tuning to longer wavelengths beyond $1100 \mathrm{~nm}$ proved to be difficult, no such devices were fabricated.

SK-QDs can be more easily tuned to longer emission wavelengths and were chosen for devices emitting at $1040 \mathrm{~nm}$ and $1220 \mathrm{~nm}$. To compensate for the lower peak gain, the SK-QDs were tuned to a longer emission wavelength than the $1040 \mathrm{~nm}$ target wavelength to benefit from higher gain of ES transitions. In consequence, peak GS emission was at $\approx 1120 \mathrm{~nm}$ to enable emission from the $1^{\text {st }} \mathrm{ES}$ at the target wavelength of $1040 \mathrm{~nm}$. Wavelength shifting was achieved predominantly by altering GRI, SRL, and the capping procedure, as detailed in Chapter 4.

\subsection{2 $950 \mathrm{~nm}$ SML-VECSEL}

Realization of QDs enabling sufficient gain at the target wavelength of $940 \mathrm{~nm}$ proved to be difficult, thus VECSEL devices for this wavelength are solely based on SML structures. SML emission is tuned to a shorter wavelength emission of $\approx 920 \mathrm{~nm}$ at room temperature. This compensates for the red shift caused by the elevated gain temperature upon laser operation which can attain $\approx 80^{\circ} \mathrm{C}$. Each SML structure consists of five-fold SL of $\approx 0.3$ ML of InAs and $1 \mathrm{~nm}$ GaAs. The nonlinear RPG incorporated 10 active layers positioned as two groups of 3 and two groups of 2 SML structures at the antinodes of the optical standing wave. GaAs spacer thickness within the SML-groups was $30 \mathrm{~nm}$. A schematic of the whole design is given in Figure 6.4 .

Lasing was achieved at $950 \mathrm{~nm}$ - close to the target wavelength of $940 \mathrm{~nm}$. Operation in $\mathrm{CW}$ mode up to $500 \mathrm{~mW}$ was demonstrated without signs of thermal rollover and was limited by the available optical pump power. Lasing threshold of $35.4 \frac{\mathrm{kW}}{\mathrm{cm}^{2}}$ and slope efficiency of only $3.8 \%$ were measured with a $1 \%$ OC. These values from this first VECSEL design cannot yet compete with characteristics of established and highlyoptimized conventional QW based VECSELs [Kim07]. The high threshold and low efficiency of the present device are partially attributed to the $10 \mathrm{~nm}$ offset compared to the design wavelength, but also to the limited pump light absorption within the short gain 


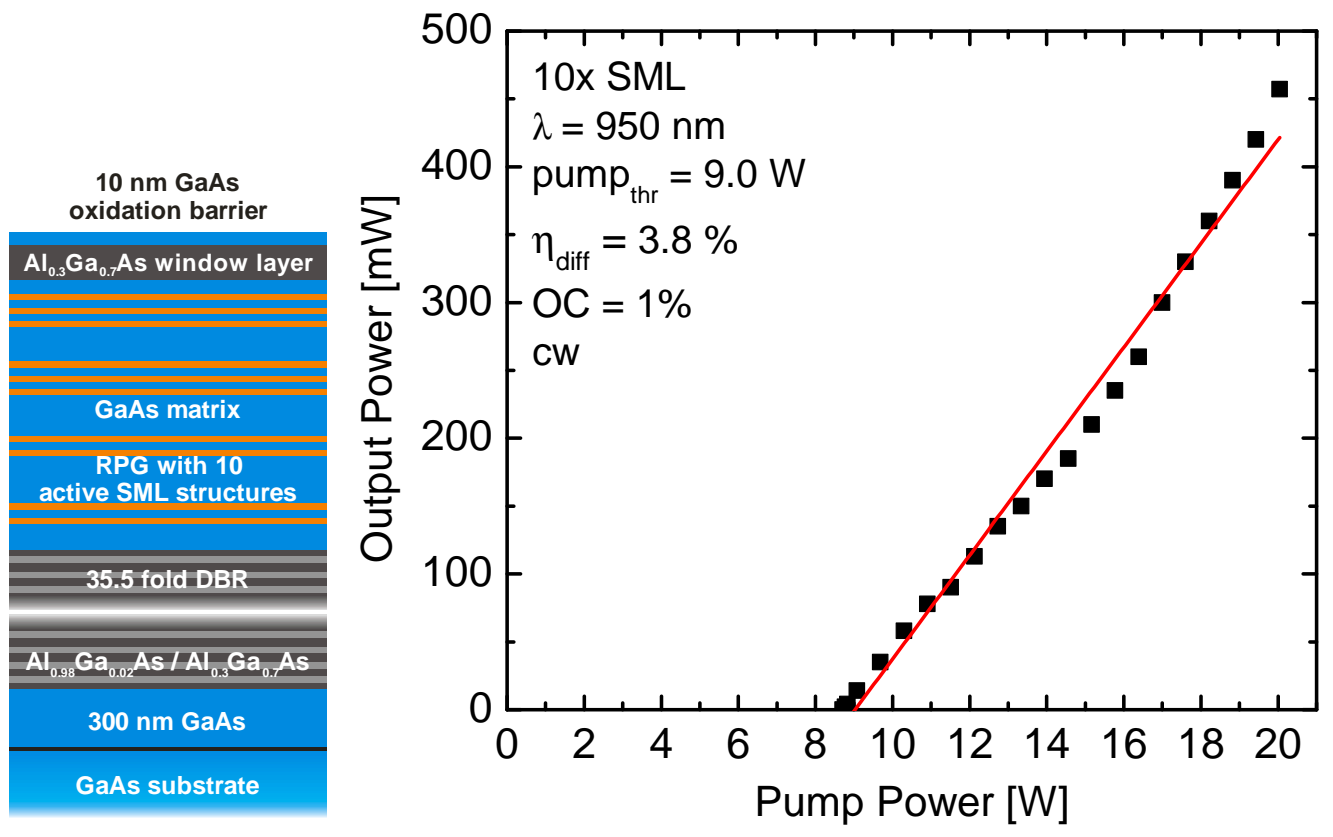

Figure 6.4: left: Schematic of the grown VECSEL design employing 10 active SML structures within its non-linear RPG section. right: Measured optical input-output data in $\mathrm{CW}$ operation mode at $15^{\circ} \mathrm{C}$ heat sink temperature.

region of only $2.25 \times \lambda$, corresponding to $0.6 \mu \mathrm{m}$ at $940 \mathrm{~nm}$ with its 10 active layers. This results in only $\approx 54 \%$ absorbed pump power as is calculated in the following:

The absorption coefficient $\alpha$ of GaAs at the wavelength of the incident pump light $(808 \mathrm{~nm})$ was calculated with extinction coefficient $k$ values taken from the "Handbook of Optical Constants of Solids" [Pal97]:

$$
\alpha=\frac{4 \pi \cdot k}{\lambda} \approx 1.3 \frac{1}{\mu m}
$$

This value is in agreement with the data from Casey et al. [HCC75]. Intensity loss within the active RPG section of the incident pump power $I_{0}$ can be calculated with:

$$
I(x)=I_{0} \cdot e^{-\alpha x}
$$

In consequence only about $54 \%$ of the applied optical pump power was absorbed within the RPG section. As the DBR is transparent for the pump light, all excess pump power was absorbed within the GaAs substrate, causing heating of the gain-chip. Especially for this relatively short wavelength future designs need to improve pump power absorption and include a larger number of active layers to improve lasing parameters. Device results are published in [Ger08a]. 

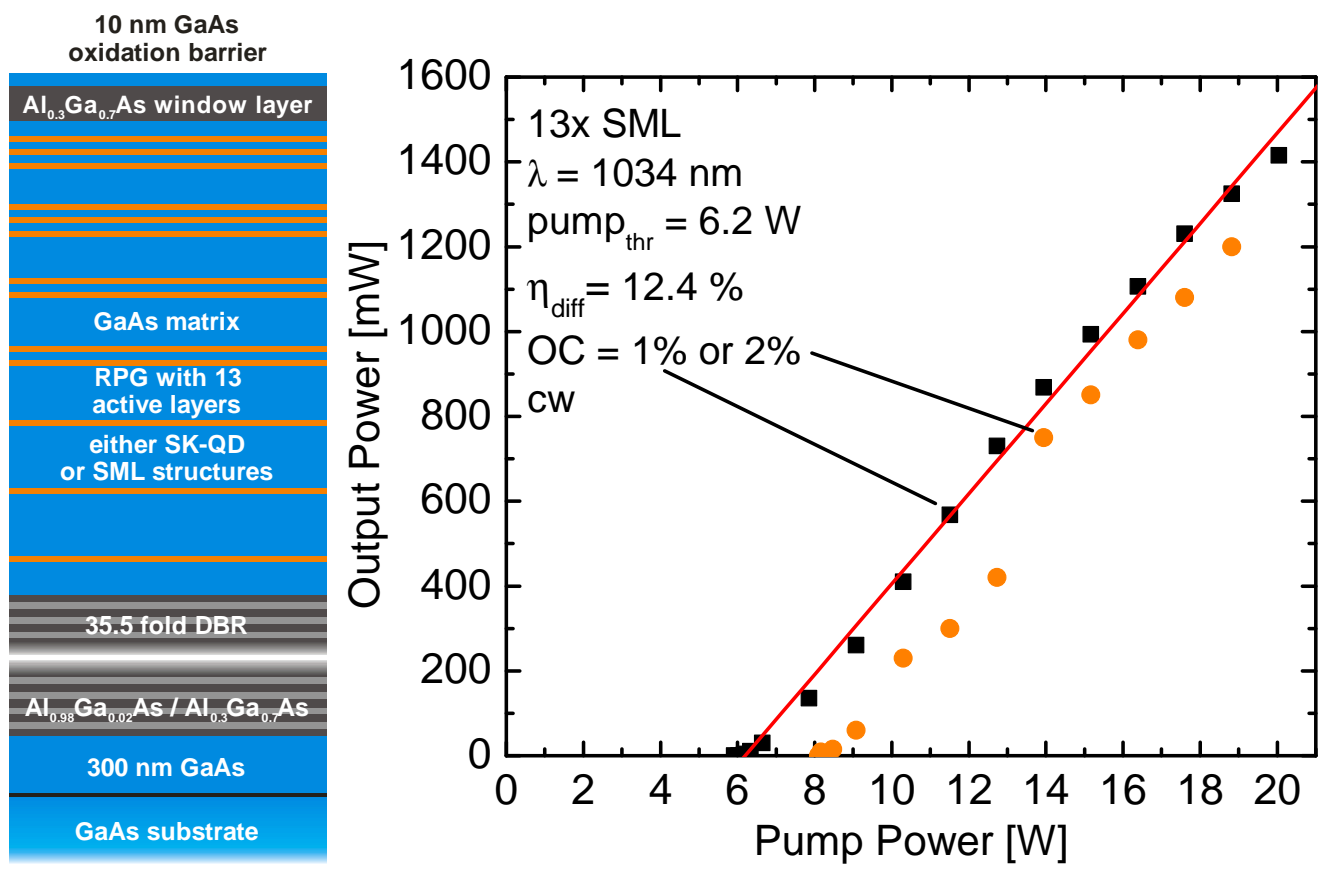

Figure 6.5: left: Schematic of the grown VECSEL design employing 13 active SML structures within its non-linear RPG section. right: Measured optical input-output data in CW operation mode at $15^{\circ} \mathrm{C}$ heat sink temperature.

\subsection{3 $1040 \mathrm{~nm}$ SML-VECSEL}

In comparison to the $940 \mathrm{~nm}$ SML-VECSEL design, the $1040 \mathrm{~nm}$ SML-VECSEL design includes 3 additional active layers each positioned at individual antinodes. Consequently, the optical length of the RPG section was increased to $3.75 \times \lambda$, and in absolute values this was almost doubled due to the longer $\lambda$ emission wavelength. This longer absorber section absorbs $\approx 77 \%$ of the incident pump light power. A schematic of the complete gain-chip structure and the laser characteristics are given in Figure 6.5.

Prior to mounting, characteristics of the SML gain-chip were assessed by PL and surface reflectivity measurements to ensure error free epitaxial growth of the VECSEL structure. Resulting PL curves represent a convolution of the DBR reflectivity, the sub-cavity formed by the DBR and the semiconductor/air interface, and the emission of the SML structures. The OD0 PL shown in Figure 6.6 is recorded normal to the sample surface at RT (black line) and at the estimated device operation temperature (OT) (red line). Gain-chip emission spectrally red shifted $44 \mathrm{~nm}$ upon heating to a perfect match with the stop band of the DBR at estimated OT. Measured reflectivity (dashed line) of the whole gain-chip structure is in excellent agreement with the simulated reflectivity using the transfer matrix method 


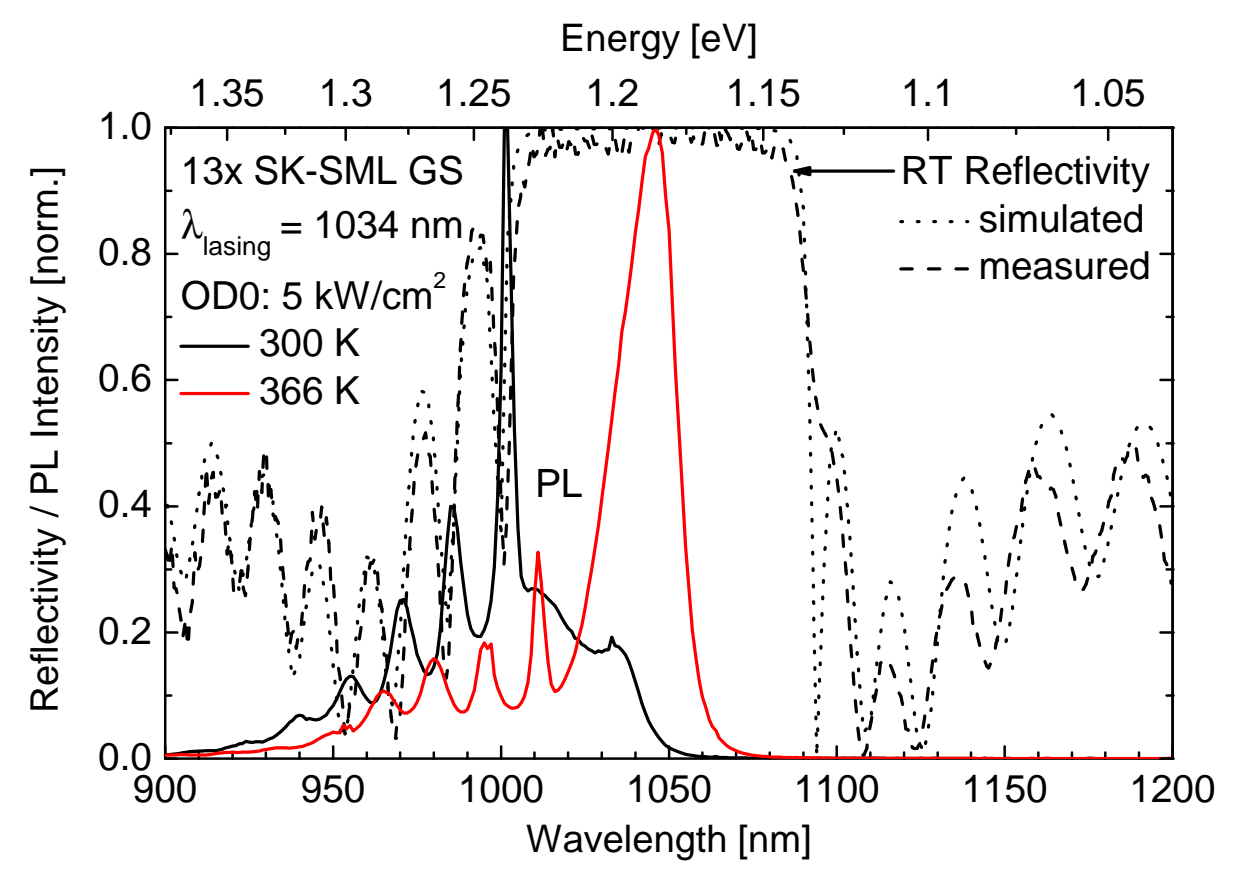

Figure 6.6: solid line: PL measurements of the final gain-chip wafer at RT (black) and at the estimated VECSEL OT (red). Heating to OT red shifts peak emission by $44 \mathrm{~nm}$, which is defined by a convolution of the spectral position of the SML emission and the cavity resonance.

dashed line: Reflectivity measurement of the final gain-chip wafer shows the $\approx 80 \mathrm{~nm}$ broad stop band of the DBR.

dotted line: Transfer matrix simulation of the complete gain-chip structure is in good agreement with the experimental data.

(dotted line). Consequently, PL and reflectivity measurements can be used as a fast quality check for the epitaxial structure prior to mounting and device operation.

Laser emission was achieved around $1034 \mathrm{~nm}$, representing an optimum wavelength to achieve a large color gamut for frequency-doubled green in RGB displays. The lasing spectrum given in Figure 6.7 shows fringes due to the etalon effect of the diamond intra-cavity heat spreader. Using $1 \%$ outcoupling, a maximum $\mathrm{CW}$ output power of $1.4 \mathrm{~W}$, almost without thermal rollover, was observed for a pump power of $20 \mathrm{~W}$. The sample delivers sufficient gain to enable the use of a $2 \%$ OC, resulting in an $\approx 25 \%$ increase in the lasing threshold, and showing similar slope efficiency (cf. Figure 6.5). The corresponding optical-to-optical conversion efficiency was $7 \%$ with $12.4 \%$ slope efficiency. These values approach well those of excellent QW-based devices [Här06]. The higher number of active layers combined with the increased pump light absorption enabled an almost three-fold increase in maximum output power and a more than three-fold increase in the slope efficiency, while reducing the lasing threshold from 35.4 to $24.4 \frac{\mathrm{kW}}{\mathrm{cm}^{2}}$ 


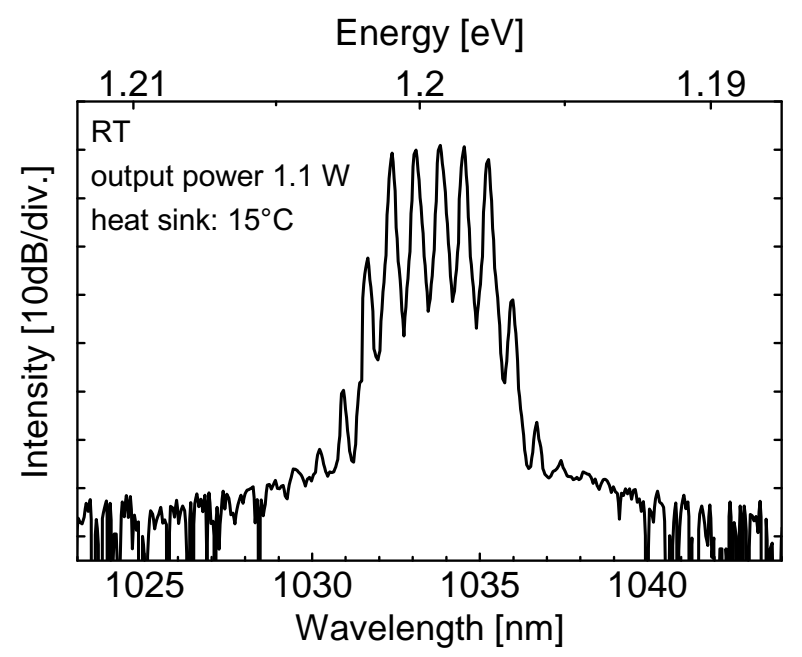

Figure 6.7: Laser spectrum of the 13-fold SML VECSEL operating at $1.1 \mathrm{~W}$ output power with a $1 \%$ OC. Fringes originate from the etalon effect of the diamond intra-cavity heat spreader. The spectrum is exemplary for all devices using this setup (cf. Figure 6.1 .

compared to the thinner SML-VECSELs emitting at $950 \mathrm{~nm}$. These results are published in [Ger08a, Ger08b].

\subsection{4 $1040 \mathrm{~nm}$ Stranski-Krastanow QD-VECSEL}

A first proof-of-principle that QDs were suitable was provided by removing the p-DBR of a MBE grown QD-VCSEL and thereby converting the structure to a QD-VECSEL by Lott et al. [Lot05]. In this work, the first MOVPE grown QD-VECSEL is presented. Through the realization of SML-VECSELs and SK-QD-VECSELs at $1040 \mathrm{~nm}$, a direct comparison of both gain media types for VECSEL applications becomes possible. Gain-chip design of the SK-QD device is identical to the SML-based active region. In contrast to the SML active layers, peak emission of the SK-QDs is tuned to an $80 \mathrm{~nm}$ longer wavelength than the target laser emission. Consequently, the device benefits from higher gain of the $1^{\text {st }} \mathrm{ES}$, allowing for twice as many possible transitions per QD. Figure 6.8 shows a comparison of both gain profiles measured with characteristic PL test samples. A dramatic difference in the spectral gain profile was observed at OD0 high excitation. SML luminescence exhibited a pronounced gain peak, which significantly shifted upon heating, while the SK-QD luminescence was spectrally very broad with FWHM $\approx 157 \mathrm{~nm}$ at RT, becoming even broader at OT FWHM $\approx 225 \mathrm{~nm}$. This resulted in an almost flat gain profile formed by the combined luminescence of GS and ES transitions from the SK-QD ensemble. For comparison, the two vertical lines point out the spectral position of the VECSEL RPG subcavity resonance at RT and OT. As SK-QDs are very sensitive to high growth temperatures and V/III-ratio during overgrowth, the integration of this 13-fold stack is especially challenging. After the growth of the 35.5-fold DBR, more than $4 \mathrm{~h}$ of additional growth time are required for the complete RPG structure which includes 13 SK-QD layers. Despite this long-lasting growth process, no blue shift of the SK-QD GS-luminescence 


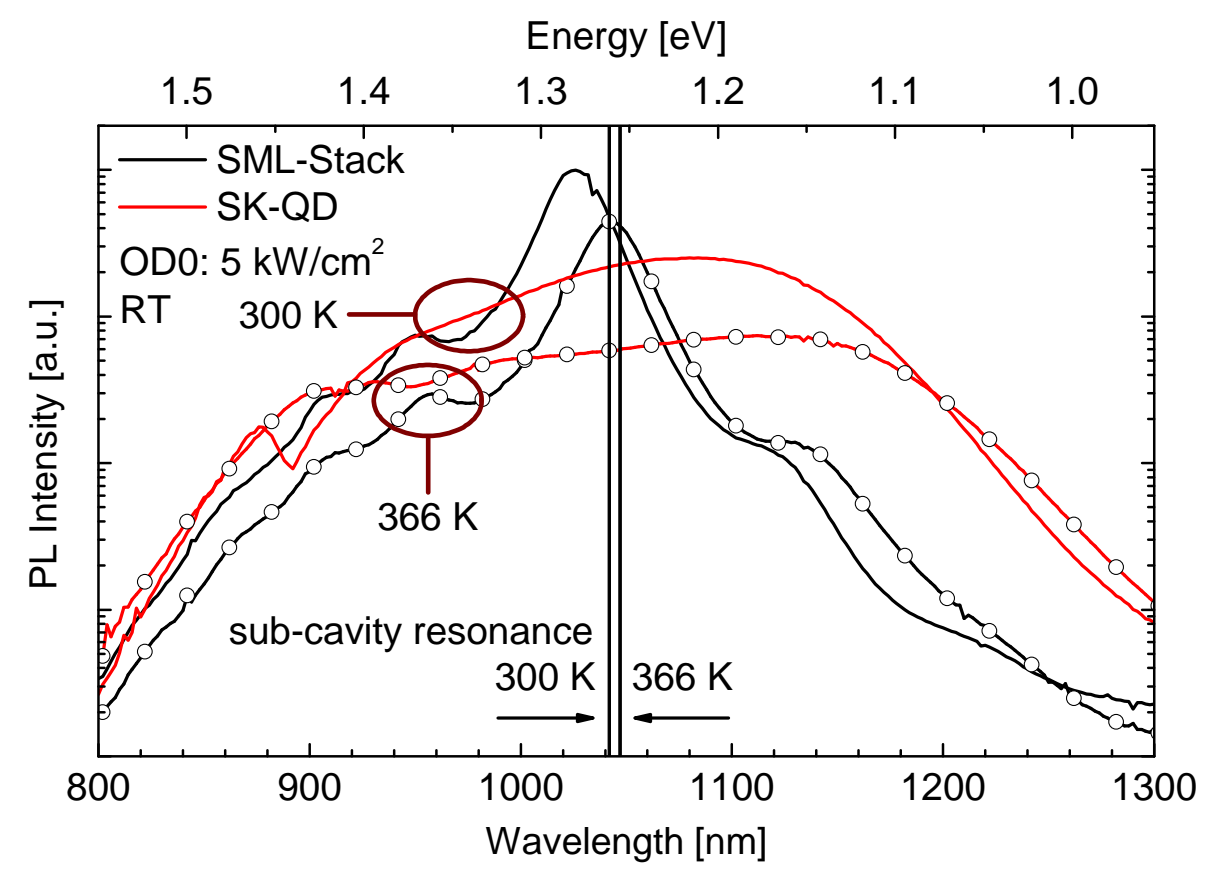

Figure 6.8: PL measurements of test samples with three-fold active layers either SML-structures (black) or SK-QDs (red). FWHM is clearly different at both temperatures.

straight lines: Measurements of both structures at RT shows misaligned peak emission of SMLstructures, whereas broad SK-QD emission already matches the sub-cavity resonance.

dotted lines: Measurements at the estimated VECSEL OT. Peak SML emission shifts into resonance while SK-QD emission matches resonance from RT to OT.

vertical lines: Lines indicate the spectral position of the sub-cavity resonance formed by the RPG section from DBR to semiconductor-surface. Heating from RT to OT causes a red shift of $\approx 5 \mathrm{~nm}$.

was observed. This spectral stability of emission was achieved by employing the improved growth parameters described in Section 5.2.1.

Measurements from the complete SK-QD gain-chip structure are depicted in Figure 6.9 demonstrating the significant difference in SK-QD compared to SML gain media shown in Figure 6.6. Peak luminescence of the gain-chip already perfectly matched reflectivity of the integrated DBR at RT. The observed red shift upon heating to OT was limited to only $6 \mathrm{~nm}$, and was synchronous to the shift of the DBR stop band. Due to the broad gain spectrum of the SK-QDs, this shift originated exclusively from the shifted resonance of the RPG subcavity, and was not defined by the spectral shift of the active medium GS emission. In consequence, the broad SK-QD gain always matched the RPG resonance condition in a manner that was largely independent of the gain-chip temperature.

This first MOVPE grown SK-QD VECSEL showed lasing operation precisely at the target wavelength of $1040 \mathrm{~nm}$. Figure 6.10 shows the device characteristics and depicts a schematic of the gain-chip structure. A pump power threshold of $24 \frac{\mathrm{kW}}{\mathrm{cm}^{2}}$ with an $1 \%$ OC 


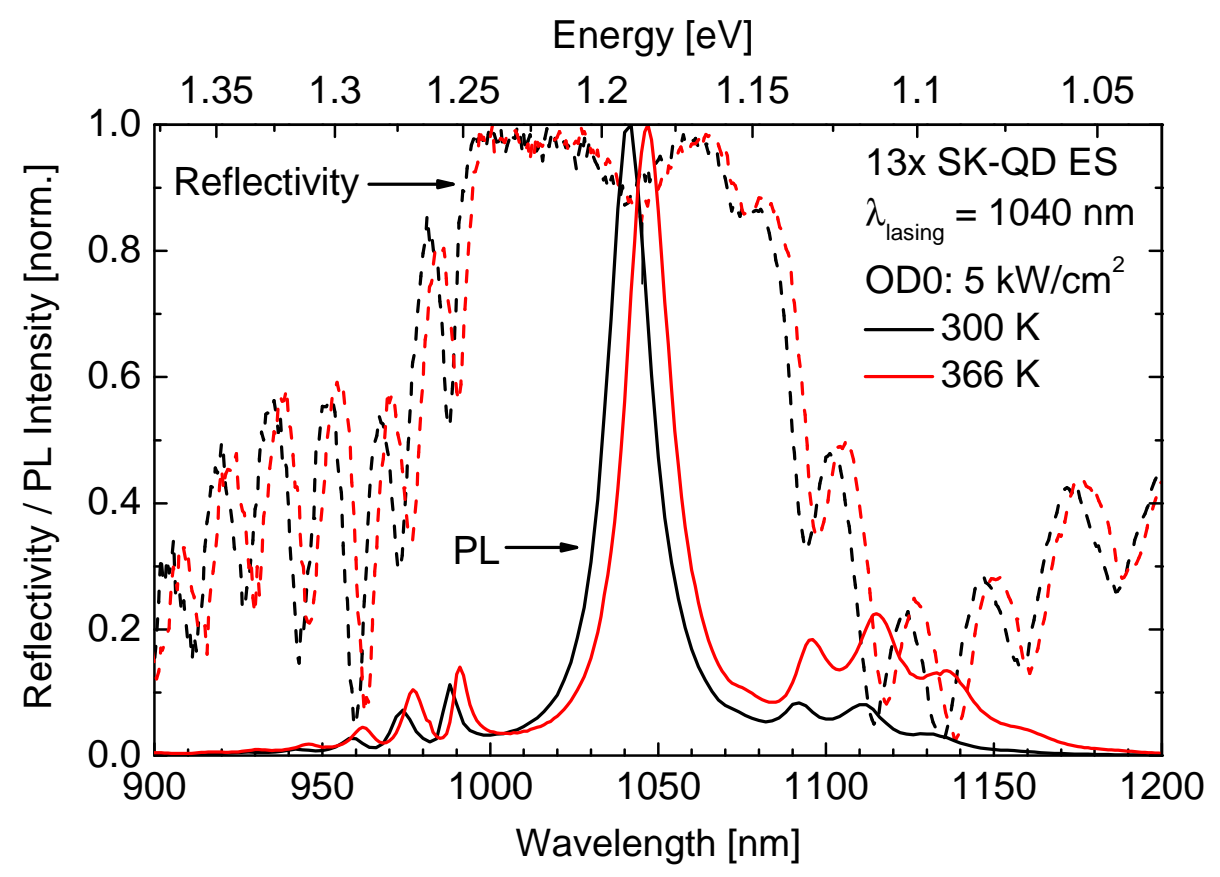

Figure 6.9: solid line: PL measurements of the final SK-QD gain-chip wafer at RT and at the estimated VECSEL OT. Peak PL emission exhibits only a minor shift of $6 \mathrm{~nm}$ upon heating originating from the subcavity resonance shift. dashed line: Reflectivity measurements of the final gain-chip wafer show the $\approx 80 \mathrm{~nm}$ broad stop band of the DBR at RT and at OT. Maximum optical SK-QD gain matches the resonance of the RPG structure at any chosen temperature.

is similar to the SML-VECSELs at the same wavelength. This implies that GS operation of QDs would allow for a threshold reduction compared to similar QW devices. Slope efficiency of $6.7 \%$ was moderate compared to the 13-fold SML-VECSELs, but almost twice as efficient as the 10-fold SML-VECSEL, both operating at GS emission. Limited efficiency of the QD device is attributed to the existence of WL states and the energetic proximity to the $G a A s$ matrix, enabling carrier escape at high temperatures. Around twice the threshold pump power a thermal rollover was observed at close to $0.3 \mathrm{~W} \mathrm{CW}$ output power. This thermal limitation is attributed to heating of the whole RPG section, thus increased kinetic energy of charge carriers. Multiple mechanisms are likely to promote parasitic losses and/or optical gain reduction. Increased phonon-assisted non-radiative recombination of charge carriers occurs within the matrix material, causing parasitic losses and additional heating of the gain-chip. For the shallow confining potential of In $G a A s$-QDs within GaAs matrix material, WL and barrier states were assumed to play an important role for luminescence decrease with increasing temperature [Pol99, Pat99]. For the present device, higher temperatures caused a red shift of active layer emission, which was significantly stronger than the resonance shift. This leads to a reduced gain originating from the $1^{\text {st }}$ ES and increased contribution from higher-level ES. Increased 

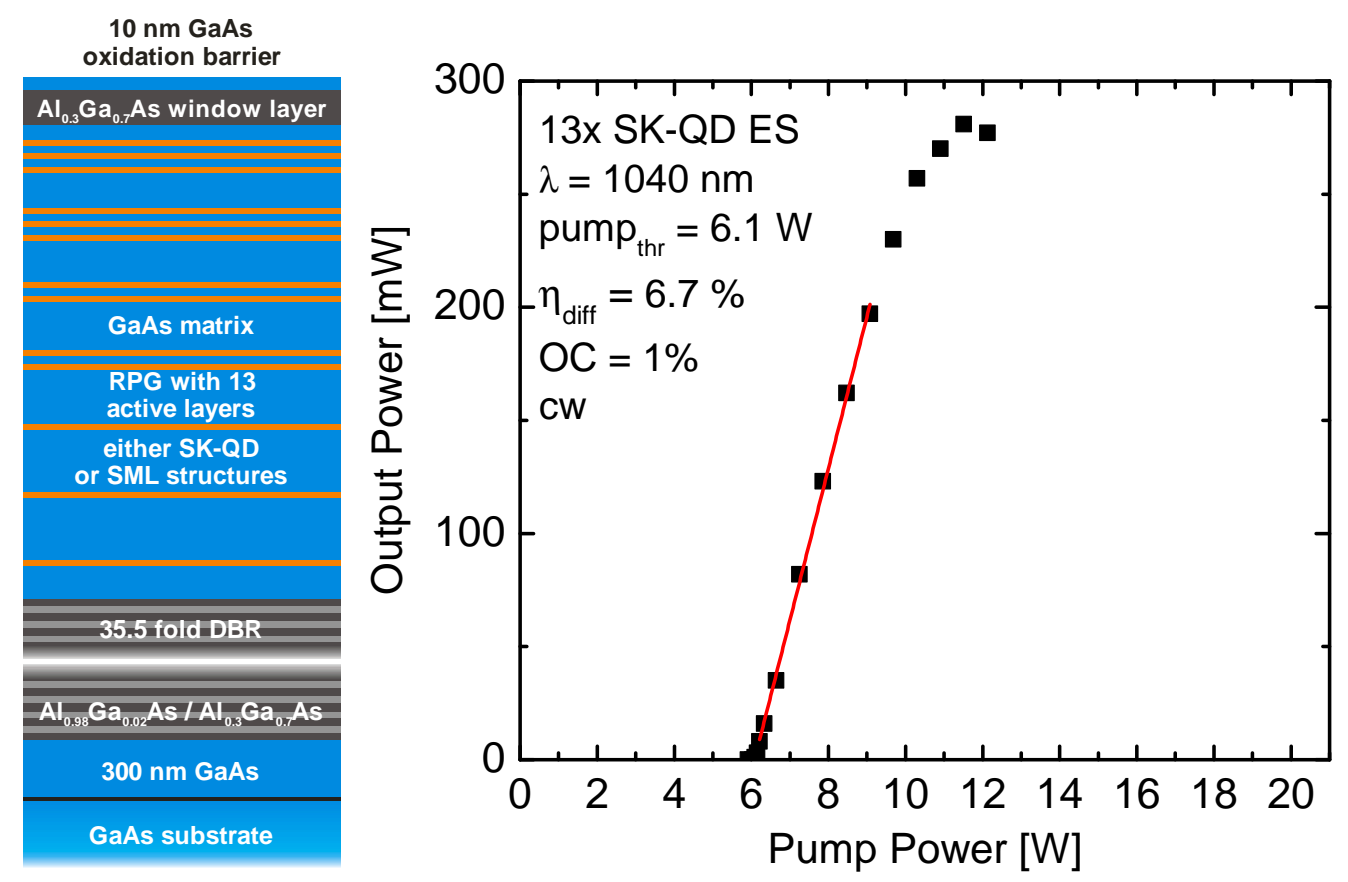

Figure 6.10: left: Schematic of the grown VECSEL design employing 13 active SK-QD layers within its non-linear RPG section. right: Measured optical input-output data in $\mathrm{CW}$ operation mode at $15^{\circ} \mathrm{C}$ heat sink temperature. RPG incident pump light absorption is calculated to $\approx 77 \%$.

kinetic energy from charge carriers enabled transitions of bound electrons from higher level ES via WL states into the GaAs conduction band. Energy calculations for electronic properties of $\operatorname{In}(\mathrm{Ga}) A s$-QDs from Schliwa et al., and experimental determination at RT of InAs-QD energy levels by Granados et al., indicate an energy offset between WL and $2^{\text {nd }}$ ES of less than $50 \mathrm{meV}$ [Gra05, Sch07]. Measurements at RT of the WL itself show an energetic distance to the $G a A s$ conduction band of only $55 \mathrm{meV}$ [Gra05]. A complete model for the SK-QD temperature dependence by thermal excitation of electron-hole pairs via the WL state to GaAs quenching states is given by Sanguinetti et al. [San99]. In contrast SML-based structures do not include a WL energetic level, thus the required energy for bound charge carriers to escape the binding potential is significantly increased [Xu96].

This conclusion is further supported by PL measurements from the employed SK-QDs at RT and at estimated OT, which are depicted in Figure 6.11. For OD1 excitation, density PL curves showed a clear separation between the GaAs band edge and the active layer luminescence. The measurement at highest excitation density OD0 resulted in a further increased sample temperature and charge carrier density, causing a continuous density of states at OT from the GaAs band edge to the SK-QD GS emission. No luminescence dip 


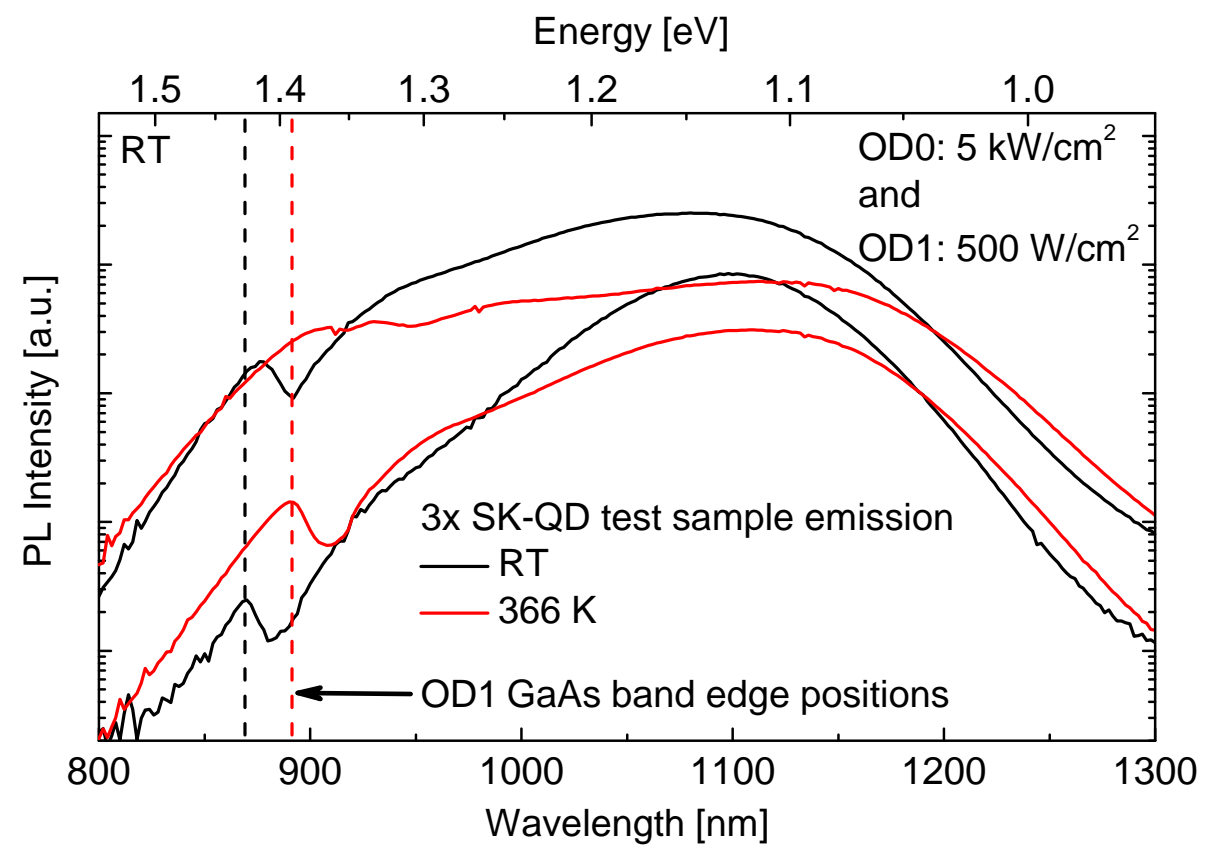

Figure 6.11: solid lines: Threefold SK-QD PL test sample measured at RT (black) and estimated OT of the VECSEL (red) at OD1 and OD0 high excitation densities.

vertical dashed lines: Indication of the OD1 spectral positions of the GaAs band edge luminescence which red shifts $22 \mathrm{~nm}$ upon heating.

At OD0 excitation density the $G a A s$ band edge positions shows $8 \mathrm{~nm}$ additional red shift, indicating heating caused by the increased excitation laser absorption. Peak luminescence of the GaAs band edge is well separated from active layer emission for all curves except for OD0 excitation density at OT which shows an almost flat, extremely broad luminescence. This indicates a continuous density of states from GaAs band edge to the SK-QD GS emission within this sample.

separating the GaAs luminescence was visible for the OD0 measurement at 366K. As the PL measurement is a convolution of the density of states with the charge carrier density, the continuous luminescence evidences the possibility of charge carrier transfers from the

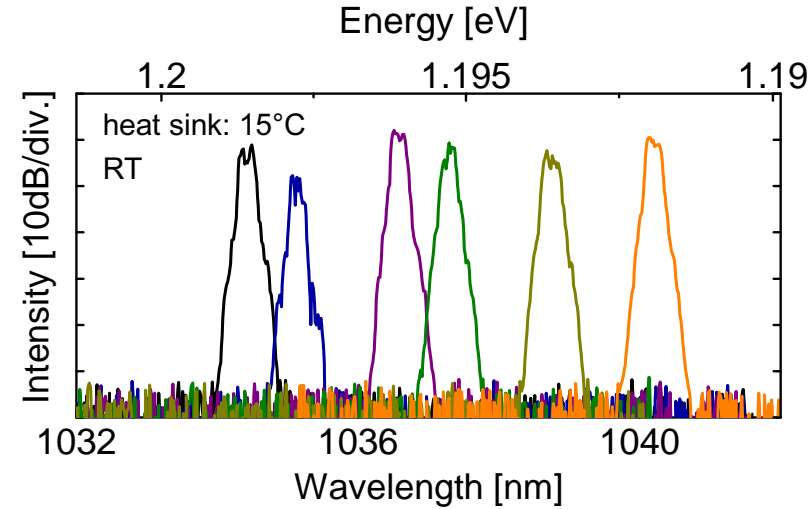

Figure 6.12: Intra-cavity tuning of the operation wavelength. Tuning range is limited by the employed etalon. 
Figure 6.13: main: Input-output curve of the 13-fold SML-VECSEL measured at IOP with $60 \mu \mathrm{m}$ pump spot diameter. inset: Laser spectrum shows fringes originating from a 250 $\mu \mathrm{m}$ thick diamond heat spreader.

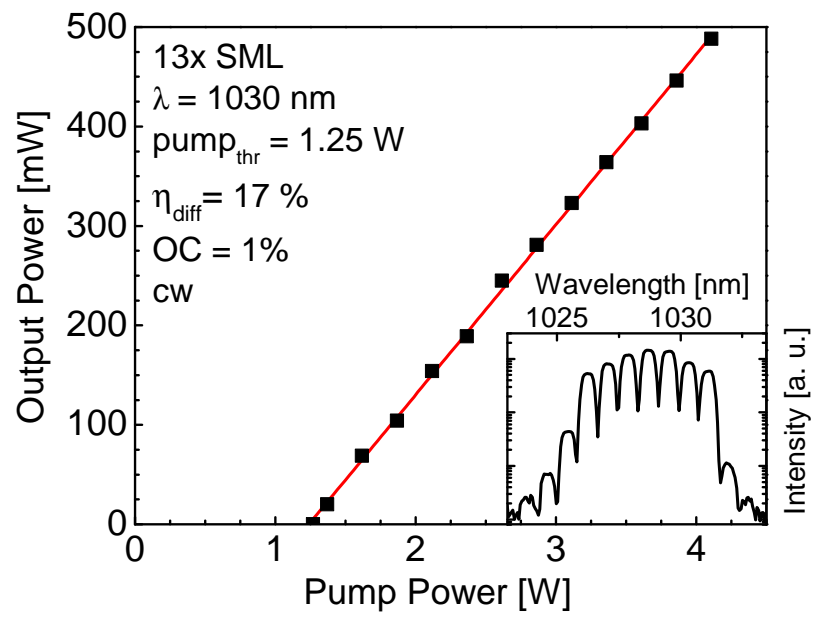

QD states to the $G a A s$ matrix. In consequence, electrons needed for the lasing operation were delocalized from the bound holes within the QDs. Thusly, a momentum offset further inhibited charge carrier recombination, and thermal rollover upon laser operation was promoted. Despite the limitations of these first QD-based VECSELs, intrinsic advantages could already be clearly evidenced from PL measurements. The emission wavelength shift with temperature was no longer set by the spectral active layer position, but rather defined by the cavity resonance. Consequently, a more than four-fold reduction in the thermal shift of $0.075 \mathrm{~nm} / \mathrm{K}$ was seen, as compared to QW-based structures[Zak05]. Additionally, the broad spectral gain allows for easy emission wavelength tuning, which was tested by insertion of a $25 \mu \mathrm{m}$ thick intra-cavity etalon. Tuning from $1034 \mathrm{~nm}$ to $1040 \mathrm{~nm}$ was possible with constant output power levels but was limited by the etalon wavelength (cf. Figure 6.12]. Results obtained using this device have been published in [Str08b, Ger08a].

\subsubsection{Temperature dependence and lifetime}

As part of the NATAL project the $\mathrm{IOP}^{2}$ performed temperature-dependent and lifetime measurements of the VECSEL structures grown at TUB. These results were first presented at CLEO 2008 [Sch08]. The IOP VECSEL setup is very similar to the setup at ORC (Vshape cavity, roc $=100 \mathrm{~mm}, 1 \%$ OC, $808 \mathrm{~nm}$ pump laser, $250 \mu \mathrm{m}$ diamond heat spreader) but employs a much smaller $60 \mu \mathrm{m}$ pump spot diameter (cf. Figure 6.1). As a consequence, VECSEL characteristics are altered. In particular, a higher pump threshold was required to achieve lasing. The aforementioned 13-fold SML-VECSEL at $1034 \mathrm{~nm}$ was mounted and measured in Tampere and also at IOP. The results showed a significant increase in threshold

\footnotetext{
${ }^{2}$ Institute of Photonics, University of Strathclyde, Wolfson Centre, 106 Rottenrow, Glasgow G4, United Kingdom
} 


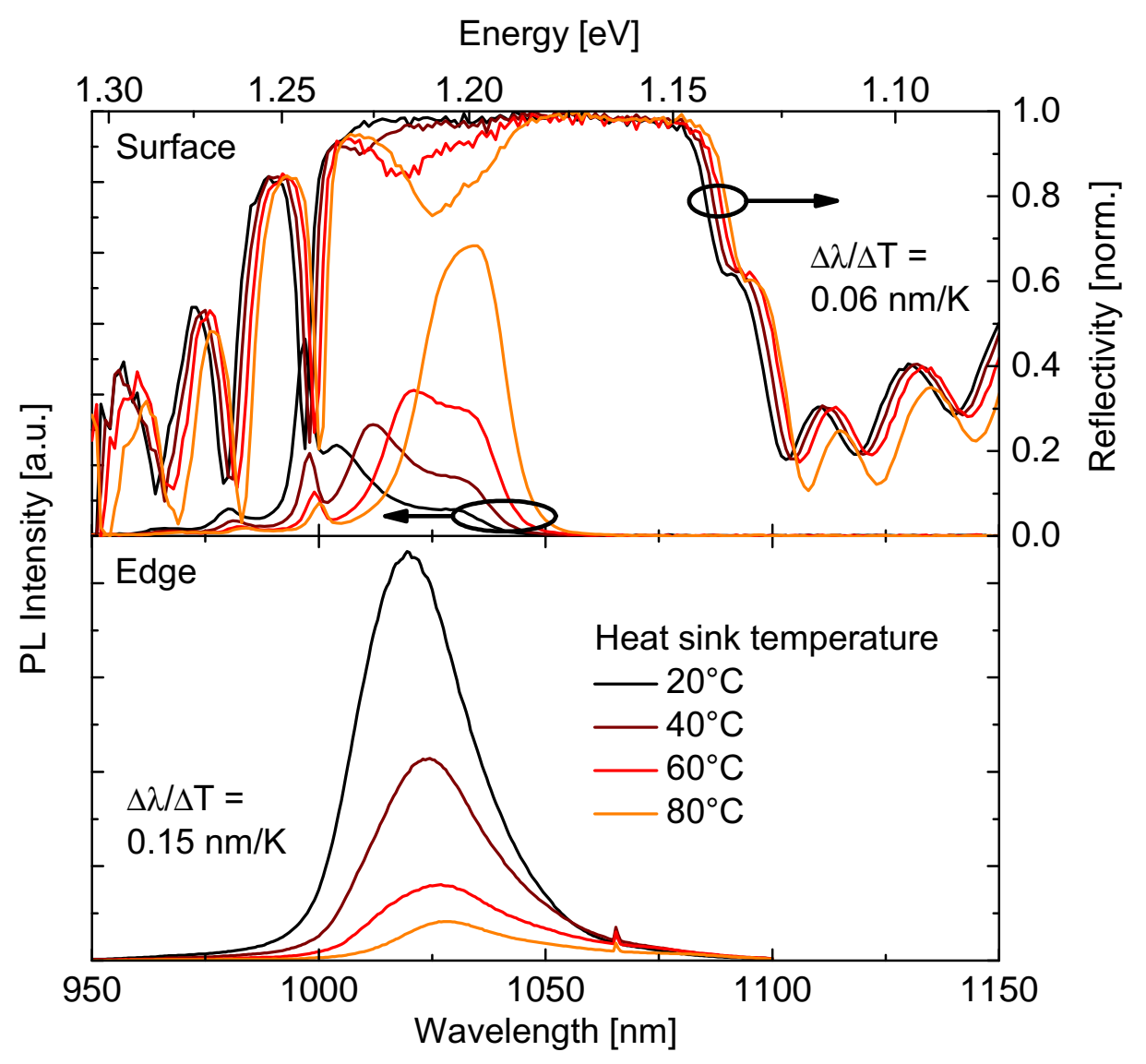

Figure 6.14: Data is measured at IOP. top: Vertical incidence surface reflectivity and PL measurements at altered heat sink temperatures are shown. Emission shifts with temperature into resonance of the RPG structure. bottom: Edge facet PL measurements of the same gain-chip show a strong decrease in intensity at higher temperatures, with gain coming into resonance.

from 24.4 to $44.2 \frac{\mathrm{kW}}{\mathrm{cm}^{2}}$ for the IOP data, and a slightly shorter emission wavelength around $1030 \mathrm{~nm}$ was measured (cf. Figure 6.13).

Detailed temperature-dependent measurements of vertical incidence reflectivity and surface PL from $20^{\circ} \mathrm{C}$ to $80^{\circ} \mathrm{C}$ are shown in the upper part of Figure 6.14. This nicely depicts the shifting of the SML emission into resonance with the RPG structure. This intended shift into resonance results from the different thermal shifts of the RPG resonance $(0.06 \mathrm{~nm} / \mathrm{K})$ and the SML emission wavelength $(0.15 \mathrm{~nm} / \mathrm{K})$. In consequence, VECSEL devices exhibit a distinct optimal OT. The investigated SML-VECSEL emission wavelength is slightly short of the $1040 \mathrm{~nm}$ target wavelength of the RPG design. Thus, device characteristics cannot represent optimal performance of the SML active medium. Additional to the surface emission and reflectivity, the measured decreasing edge emission of the gain-chip is shown for comparison in the lower part of Figure 6.14. This decrease of luminescence intensity is based on lowered PL of the SMLs at higher temperatures, and 


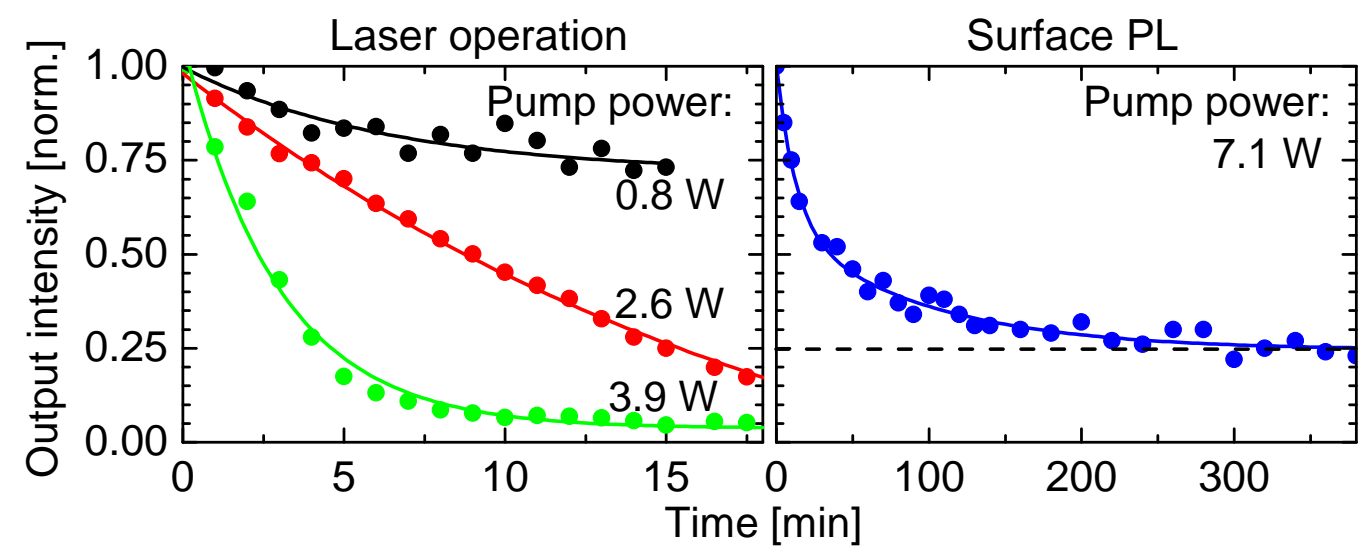

Figure 6.15: Data is measured at IOP. left: VECSEL optical output power degradation at different pump powers is recorded over time. right: Surface PL without cavity resonator at $7.1 \mathrm{~W}$ pump power shows an exponential intensity decrease. Output stabilizes at $\approx 25 \%$ of the initial intensity.

on the increasing cavity effect close to the RPG resonance, which increasingly directed towards vertical light emission.

All devices employing the described nonlinear design showed degradation during laser operation already within the ORC setup. At IOP, degradation of the 13-fold SMLVECSELs was investigated in more detail. The left part of Figure 6.15 shows the normalized output intensities of the device within the laser setup at different pump powers. The extremely high intra-cavity optical power intensities within the VECSEL setup during lasing caused strong effects on the output power over time. Initial intensity decreased rapidly (within minutes) to about $5 \%$. Below lasing threshold at $0.8 \mathrm{~W}$ pump power, a much slower and less significant degradation was observed. Laser operation ceased after several minutes and could not be recovered from the same pump spot after a break, which caused the sample to cool down. This effect represents permanent damage due to degradation of the structure upon laser operation. On the right side of Figure 6.15 the PL intensity over time is shown. This data was recorded without the cavity resonance condition, thus the sample was not exposed to the very high intra-cavity intensities upon laser operation. PL emission at the highest achievable pump power of $\approx 250 \mathrm{~kW} / \mathrm{cm}^{2}$ (equates to $7.1 \mathrm{~W}$ incident pump power) decreased too, but much slower (within hours) and stabilized after $\approx 6$ hours close to $25 \%$ of the initial output intensity. Data from the lifetime investigations suggests that degradation of device performance was due to a deterioration of the gain-chip cavity causing increased losses. Among many known semiconductor laser degradation mechanisms, which are mainly located in the proximity of the active layers [Mon81], or due to surface degradation such as excitation enhanced oxidation of GaAs [Suz77], the present results are attributed to increased defect formation within the $A l G a A s$ window and DBR layers. The gain-chip surface was protected by the bonded 
diamond heat spreader, and QD and SML active layer degradation would be required to affect both VECSEL types simultaneously. Such active layer degradation is not observed for other laser types based on the same active media growth processes. Opposite to active layers, the growth of the $A l G a A s$ layers was identical for all investigated VECSELs and incorporated a relatively high amount of carbon due to the TBAs-based process. This can lead to background doping levels up to $p=1.1 \cdot 10^{18}$ (cf. Table 4.2). Degradation of similar, but intentionally doped VCSEL p-DBRs was investigated in more detail by cathodo-luminescence, electro-luminescence, and electron-micrograph measurements, and has been reported in the literature [Che95, Her95, Che96]. These publications report recombination-enhanced defect reactions within the p-DBR due to band-tail absorption via carbon acceptor levels. Carbon acceptors in $\mathrm{AlGaAs}$ exhibit binding energies around 30 $\mathrm{meV}$ [Mas83], allow for minority charge carrier generation in the current band by photon absorption. In consequence, high photon densities can cause significant minority carrier populations and thus promote defect propagation. Such effects are predominant in p-doped material due to the approximately three times higher absorption coefficient of acceptors compared to donors. Similar effects are suspected for the presented devices, as they are also exposed to the extremely high intra-cavity photon densities of the laser operation, plus the additional absorption of the $808 \mathrm{~nm}$ pump light which is close to the AlGaAs band gap energy. As DBR quality is especially crucial for low loss devices such as VECSELs, a slight reduction in the gain-chip reflectivity can cease any laser operation. In consequence, improved growth processes and designs need to take into account absorption in general, and unintentional high doping levels of the DBR in particular. 


\subsection{Long wavelength gain structure design}

Long wavelength emission beyond 1.2 $\mu \mathrm{m}$ was not achieved with SML structures, and ES operation of SK-QDs would require GS emission close to $1.3 \mu \mathrm{m}$, which is difficult to achieve for stacks with 10 or more active layers and a high areal QD density. Thus, a novel design concept was developed to target SK-QD GS emission at $1220 \mathrm{~nm}$. Changes were aimed at loss reduction, while increasing pump light absorption and number of active layers in order to compensate for the inferior gain compared to the $1040 \mathrm{~nm}$ ES design. In parallel, the limited lifetime due to DBR degradation was addressed. Changes compared to the initial nonlinear gain structure design described in Section 6.2 are:

- Exchange of the TBAs precursor to an arsine-based growth process for all layers except for the QD deposition to reduce unintentional carbon p-doping by about one order of magnitude, $\mathrm{cf}$. 4.2 .

- Improved gain by increasing the number of active QD layers within the RPG to 21 or 30. Groups of three active layers are positioned at all antinodes of the optical standing wave.

- Usage of an optimized and highly stackable SK-QD growth process with GS emission at $1220 \mathrm{~nm}$, including advances described in Sections 4.1 and 5.2 .

- Elimination of the absorbing GaAs oxidation cap layer and the thick $\lambda / 2$ AlGaAs charge carrier barrier by replacing both with a single, thin, lattice matched InGaP capping layer.

- DBR thickness was increased to $37.5 \lambda / 4$-pairs and employed a nominal composition of $A l_{0.2} G a_{0.8} A s$ compared to $A l_{0.3} G a_{0.7} A s$ for the high index material. Consequently, the refractive index offset and layer number increase promote DBR reflectivity, thus losses to the substrate were reduced.

These changes resulted in improved DBR parameters yielding nominal reflectivity of $99.96 \%$. Absorption of the incident power was further increased due to the longer target wavelength of $1220 \mathrm{~nm}$ and the $5.25 \times \lambda$ thick RPG section of the 30 QD layer device. For $7 \times 3$ active layers and $10 \times 3$ absorption of light within the RPG section, which is not reflected at the wafer or diamond surface, was calculated to $\approx 83 \%$ and $\approx 91 \%$, respectively.

\subsection{1 $1210 \mathrm{~nm}$ Stranski-Krastanow QD-VECSEL}

In contrast to growth of the initial nonlinear design, the epitaxial growth process of the $1210 \mathrm{~nm}$ QD-VECSEL gain-chips was changed to a two-step process to economize cost 

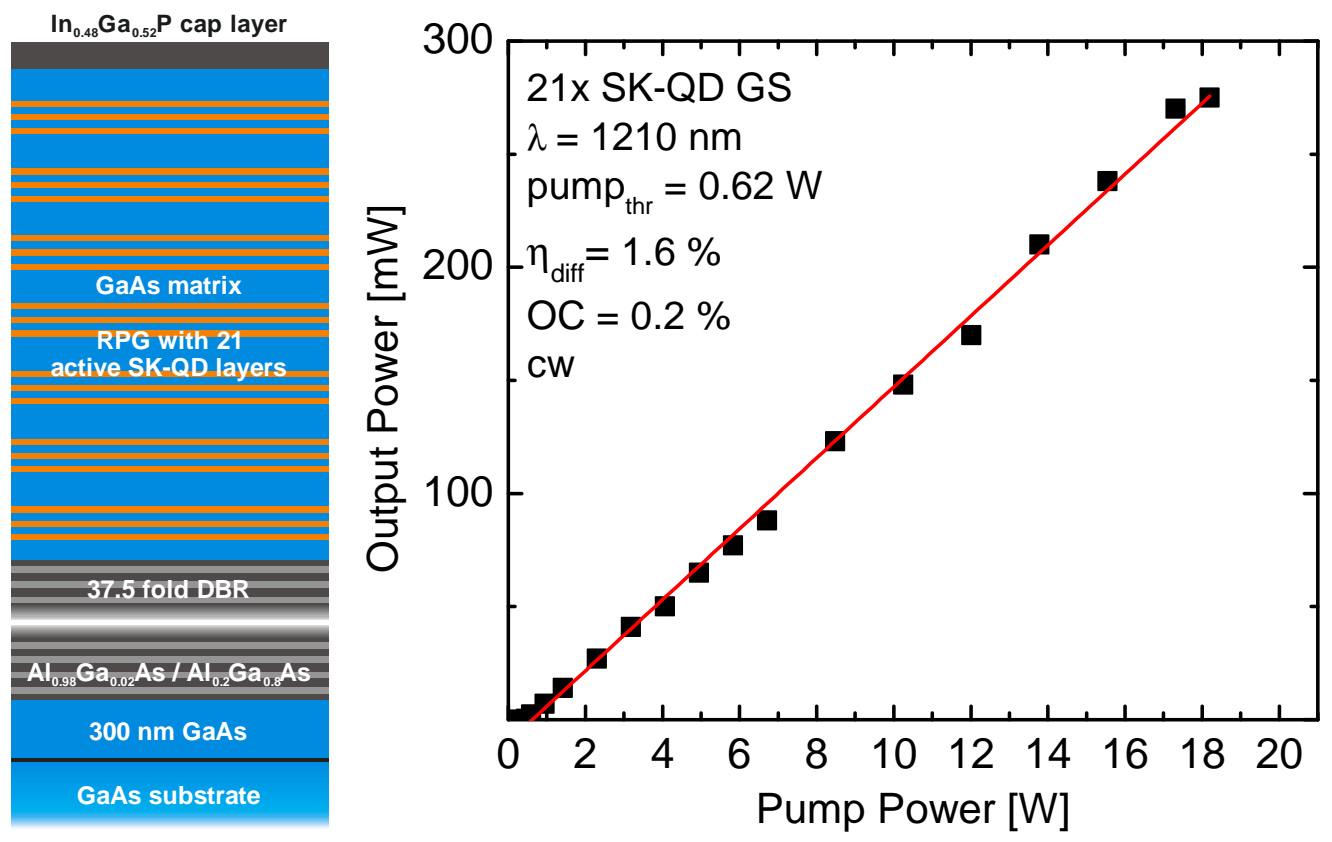

Figure 6.16: left: Schematic of the grown VECSEL design employing 21 active SK-QD layers within its RPG section. right: Measured optical input-output data in $\mathrm{CW}$ operation at $15^{\circ} \mathrm{C}$ heat sink temperature. RPG incident pump light absorption is $\approx 83 \%$.

and time for the development of different structures. The DBR part was grown on a full 2 inch wafer and capped by $100 \mathrm{~nm} \mathrm{GaAs}$. This growth run was completely carried out at nominal $700^{\circ} \mathrm{C}$, employing only arsine as group $\mathrm{V}$ precursor. Subsequently, the wafer was cleaved into quarters and each piece was individually overgrown, employing a series of varied RPG designs. Thus, both devices with 21 or 30 active layers presented within this Chapter incorporate the very same DBR and were grown on the identical wafer. For device operation, the ORC VECSEL setup was slightly altered as compared to Figure 6.1. Optical pumping was realized by a fiber-coupled diode laser delivering up to $50 \mathrm{~W} \mathrm{CW}$ power at $790 \mathrm{~nm}$, incident at $35^{\circ}$ to the surface normal. Diamond heat spreader thickness was 267 $\mu \mathrm{m}$ and a high reflectivity OC mirror was used with $99.8 \%$ nominal reflectivity.

For the first device employing the long wavelength design, the same number of standing wave antinodes within its RPG structure were chosen as for the devices operating at 1040 $\mathrm{nm}$ (Sections 6.2.3 and 6.2.4), but now 3 QD layers were placed at each antinode. Based on data from similar QDs in edge-emitters, which require a transparency current density per QD layer of $\approx 10 \mathrm{~A} / \mathrm{cm}^{2}$, the necessary incident optical pump power density for inversion of the 21-fold QD stack is estimated to $<1 \mathrm{~kW} / \mathrm{cm}^{2}$ [Ger07]. A schematic of the gain-chip structure is given in Figure 6.16, depicting the device characteristics with 


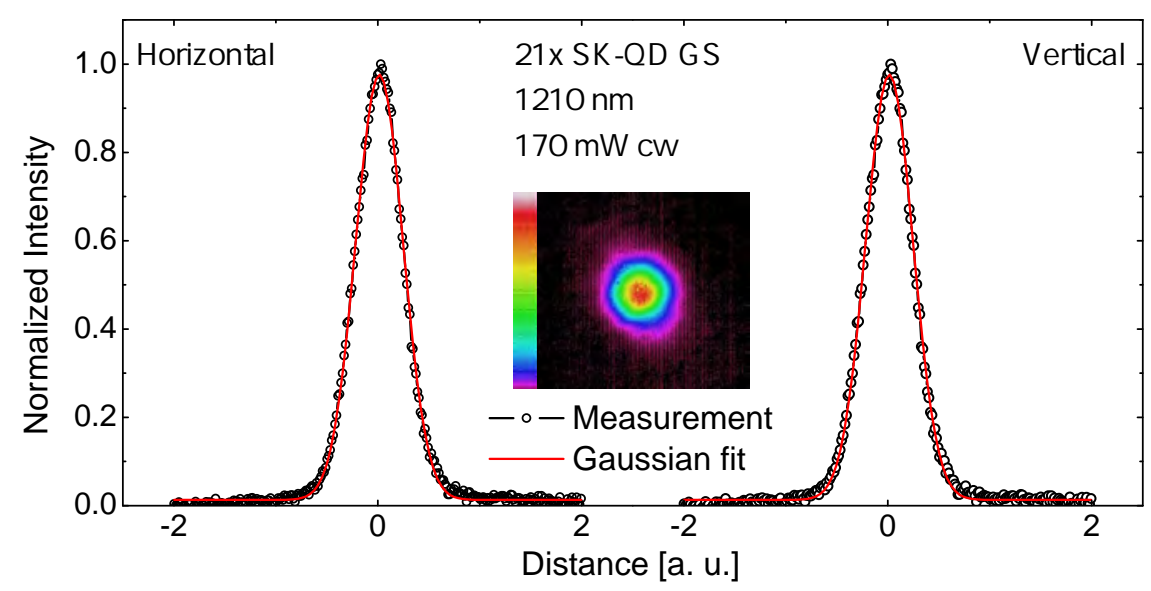

Figure 6.17: 21-fold SK-QD VECSEL operating at $1210 \mathrm{~nm}$ with a $\mathrm{CW}$ output power of $170 \mathrm{~mW}$. left and right: Measured transverse beam profiles in two orthogonal directions with corresponding Gaussian fit. inset: Spatial intensity profile measured with CCD-camera.

a very low threshold of only $2.4 \mathrm{~kW} / \mathrm{cm}^{2}$. This excellent threshold value is partially due to the employed high reflectivity OC, but was only achieved through the use of QD GS emission. Other reported values for GaInNAs or GaAsSb QW-based devices in this wavelength regime are higher, despite sophisticated technologies such as substrate removal and/or double-band DBR design [Ger03, Die07]. In contrast, the data presented here were measured without any processing. The as grown wafer was cleaved and directly mounted to the setup as shown in Figure 6.1. Thus, heat dissipation through the bottom of the gain-chip is additionally attenuated by the $\approx 400 \mu \mathrm{m}$ thick GaAs : Si wafer. Maximum $\mathrm{CW}$ output power achieves close to $300 \mathrm{~mW}$. Limitation by thermal rollover is attributed to the increased ultra-high intra-cavity power-densities caused by the employed $0.2 \%$ OC. Results almost achieved lasing threshold with $1 \%$ light outcoupling indicating that increased performance should be possible with an intermediate $0.5 \%$ OC, which was not available at time of the measurements.

Beam quality demonstrates excellent $T E M_{00}$ emission as expected for this kind of device. Beam cross-sectional data is shown in Figure 6.17, depicting the Gaussian intensity profile for the fundamental mode. Similar beam shapes were measured for all other VECSEL devices in this Chapter, and also for the nonlinear design.

To further investigate the optimal number of active layers, a second version of the linear design was grown on the same wafer, employing $10 \times 3$ active QD layers. A schematic drawing and the characteristic input-output data are shown in Figure 6.18. This thicker device showed a slightly inferior performance in terms of differential efficiency and threshold, combined with an earlier thermal rollover, limiting CW output to about 200 $\mathrm{mW}$. These changes are attributed to the high number of active layers, which were most 

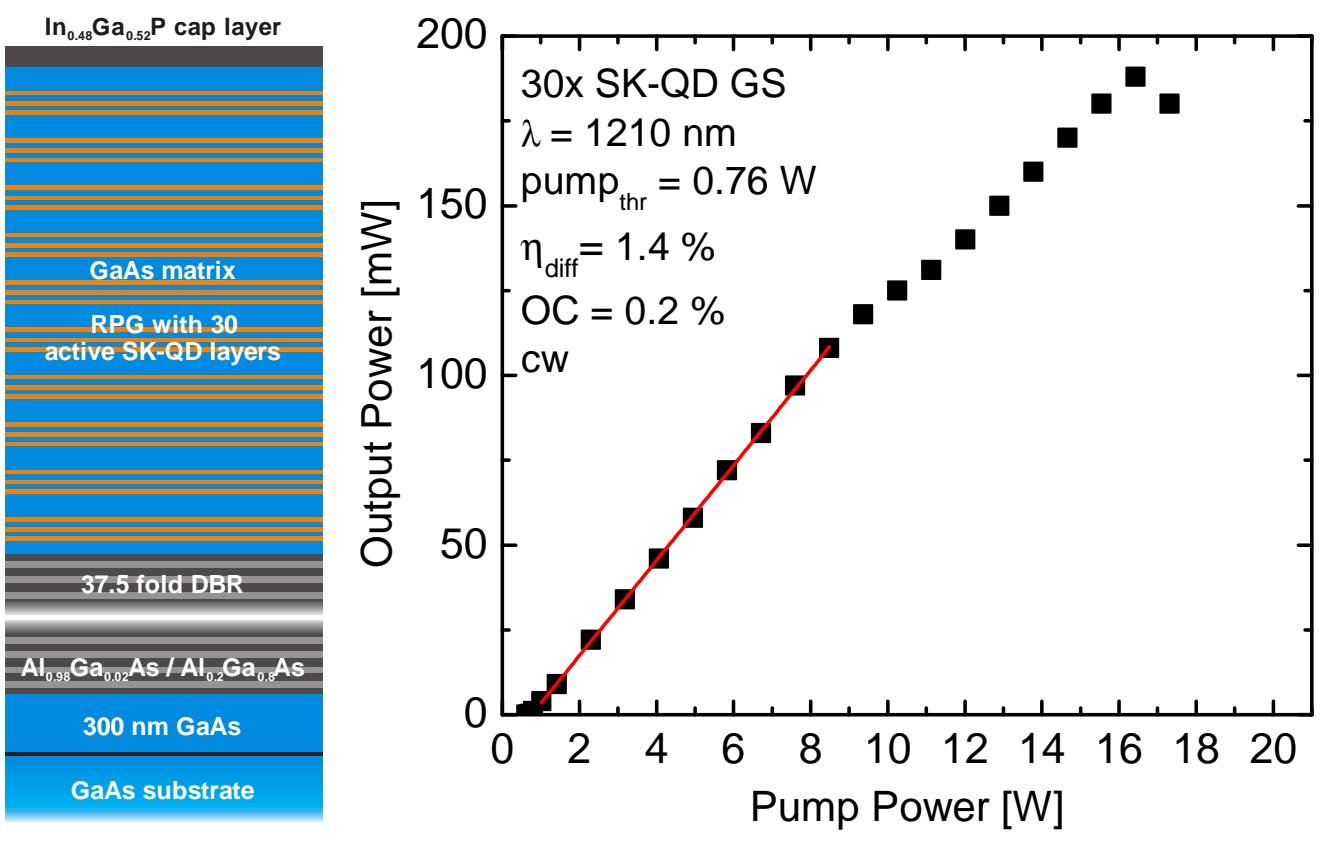

Figure 6.18: left: Schematic of the grown VECSEL design employing 30 active SK-QD layers within its RPG section. right: Measured optical input-output data in $\mathrm{CW}$ operation at $15^{\circ} \mathrm{C}$ heat sink temperature. The $5.25 \times \lambda$ active RPG section absorbs $\approx 91 \%$ of the incident pump light.

likely at the limit of homogenous pumping within this setup. Comparing both devices, the nominal absorption of pump light due to the thicker cavity increased by only $8 \%$, while the number of QD layers increased by $43 \%$. Thus the additional 9 active layers needed to achieve inversion with only $8 \%$ of the available pump power. In consequence, future QD-based VECSELs could be improved through a design attributing more equally the absorbed pump power to the individual QD layers. In other words, usage of a similarly thick RPG structure to absorb the incident light while distributing a smaller number of active layers throughout $G a A s$ matrix should lead to superior performance.

\subsubsection{Temperature stability}

Aside from the intrinsic advantages of the VECSEL concept, temperature stable performance is crucial for all applications. Upon operation, gain-chip temperature can attain values around $80^{\circ} \mathrm{C}$. Thus, device designs try to include estimated thermal effects and packaging or mounting concepts aim at temperature stabilization to perfectly match the target temperature for all operational conditions. All VECSEL concepts, including a resonant sub-cavity which enhances the effective gain, suffer from a relatively high sensitivity to operating temperature changes [Kuz99, Tro04]. Such conventional QW-based 

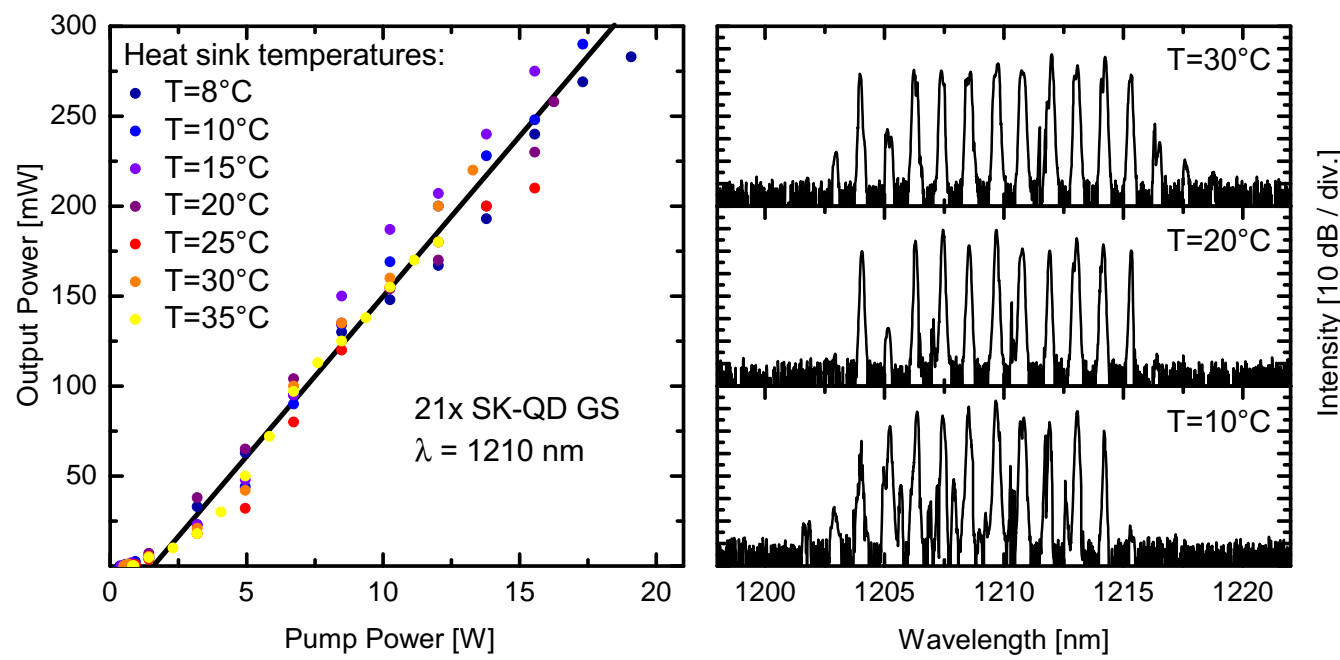

Figure 6.19: $1210 \mathrm{~nm}$ 21-fold SK-QD VECSEL operation at a series of different heat sink temperatures is shown. left: Input-output power curves at altered temperatures depict the extremely stable performance. right: Emission spectra measured at medium pump power level of $30 \mathrm{~kW} / \mathrm{cm}^{2}$ evidence the very stable emission wavelength upon temperature changes.

VECSELs operating close to the target wavelength of $1220 \mathrm{~nm}$ face additional difficulties upon growth due to the employed highly strained QWs, and exhibit strong temperaturedependent characteristics limiting optimum laser performance to a narrow temperature window [Ger03, Hop04]. This sensitivity is caused by the diverging thermal shifts of the involved components. The relatively narrow spectral emission of QW active layers shows a fast shifting behavior of $\approx 0.4 \mathrm{~nm} / \mathrm{K}$ while the sharp sub-cavity resonance of the semiconductor RPG cavity only shifts with $\approx 0.1 \mathrm{~nm} / \mathrm{K}$ [Zak05, Die07]. This about fourfold faster shift of the gain medium needs to be accounted for prior to growth to achieve gain

Figure 6.20: $1210 \mathrm{~nm}$ 21-fold SKQD VECSEL operating at constant medium pump power level but altered heat sink temperatures. top: Differential efficiencies of the device bottom: threshold pump power levels at a series of operating temperatures.

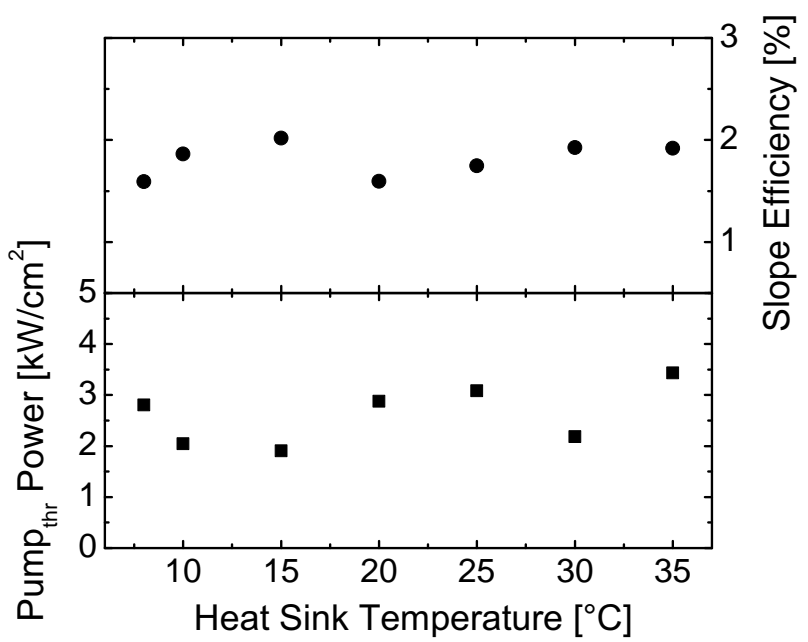




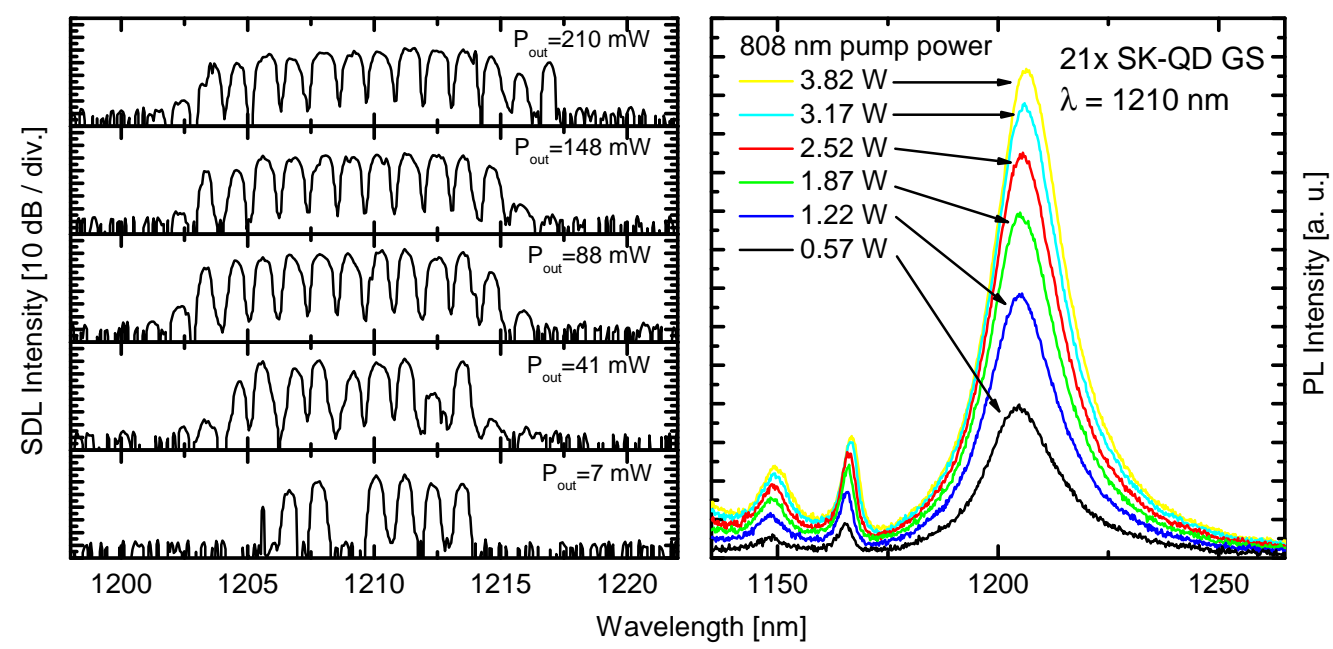

Figure 6.21: $1210 \mathrm{~nm}$ 21-fold SK-QD VECSEL operating at constant heat sink temperature of $15^{\circ} \mathrm{C}$ but altered pump power levels. left: Laser operation emission spectra reveal a perfectly stable emission wavelength over the full output power range. right: PL measurements carried out at TU Berlin (cf. 6.1.2) depict spectrally stable emission at all applied pump power levels.

and resonance matching. Consequently, only one distinct operating temperature allows for optimal performance, making devices vulnerable to external temperature changes. A deviation from this optimum temperature results in strongly inferior differential efficiencies, and altered threshold values [Ger03, Smi04, Kim06, Fan07b]. Additionally, the VECSEL emission wavelength can shift strongly upon temperature changes [Kon07, Rau08]. As the incident optical pump power is altered to change the output-power level, the heat input is also altered. This necessitates strict temperature management upon operation, and drives costs for packaged devices.

As depicted in Figure 6.8, SK-QDs offer simultaneous gain for a very broad spectral range as a result of the inhomogeneous broadening of QD ensembles in combination with luminescence from ES transitions at high pump levels. Consequently, a constant gain and sub-cavity resonance matching is given for a wide range of temperatures, despite the fact that the QD GS luminescence exhibits a similar temperature shifting behavior as QW structures. This led to extremely temperature stable operations for the fabricated QD based VECSEL devices, which show almost perfectly stable parameters for a wide range of temperatures and pump powers. Measurements in Figure 6.19 depict that no wavelength shift is observed and that input-output power characteristics remain unchanged upon changes of the heat sink temperature. The changes in differential efficiency and threshold pump power usually observed for QW-based devices upon temperature changes were not present for the developed SK-QD based VECSELs, as evidenced in Figure 6.20. Investigations on pump power effects while the heat sink is kept constant at $15^{\circ} \mathrm{C}$ 


\begin{tabular}{|c|c|c|c|c|c|c|c|c|c|}
\hline 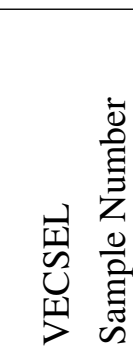 & 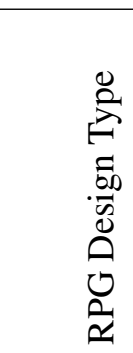 & 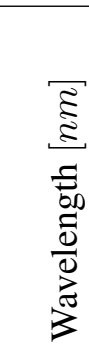 & 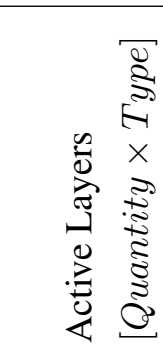 & 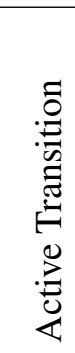 & 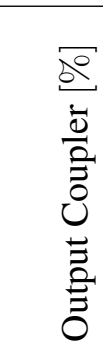 & 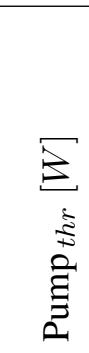 & 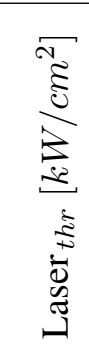 & 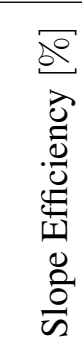 & 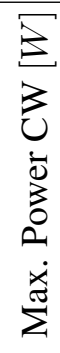 \\
\hline Np2871 & nonlin. & 950 & $10 \times \mathrm{SML}$ & GS & 1 & 9.0 & 35.4 & 3.8 & 0.5 \\
\hline $\mathrm{Np} 2862$ & nonlin. & 1034 & $13 \times \mathrm{SML}$ & GS & 1 or 2 & 6.2 & 24.4 & 12.4 & 1.4 \\
\hline Np2859 & nonlin. & 1040 & $13 \times \mathrm{SK}$ & ES & 1 & 6.5 & 24.0 & 6.1 & 0.3 \\
\hline Np3174 & linear & 1210 & $21 \times S K$ & GS & 0.2 & 0.62 & 2.4 & 1.6 & 0.3 \\
\hline Np3196 & linear & 1210 & $30 \times \mathrm{SK}$ & GS & 0.2 & 0.76 & 3.0 & 1.4 & 0.2 \\
\hline
\end{tabular}

Table 6.1: Developed and realized VECSELs at TU-Berlin within the NATAL project. Pump spot diameter for all devices is $\mathrm{d}=180 \mu \mathrm{m}$. Gain-chip heat sink is set to $15^{\circ} \mathrm{C}$

are shown in Figure 6.21. This data showed stable emission wavelength upon the full range of attainable output powers with pump powers ranging from 30 to $190 \mathrm{~kW} / \mathrm{cm}^{2}$. Only a minor shift of $\approx 0.06 \mathrm{~nm} / \mathrm{K}$, caused by the sub-cavity resonance temperature dependence was measured. Also, PL studies carried out at TUB show that emission wavelength remained stable for all available pump powers. Thus, the emission wavelength was no longer dependent on the shift of the active layers and was solely linked to the sub-cavity resonance. This means that spectrally broad gain of QD active layers solves common temperature problems such as emission wavelength stability, and heavily affects lasing characteristics. Nevertheless, good heat dissipation remains advisable for optimum QD device performance, as maximum gain decreases with raised gain-chip temperature. Despite the lower peak gain per active layer, these findings clearly depict the advantages of QD based VECSELs for industrial applications, which demand emission wavelength stability and constant performance within a wide range of temperatures. Results of these devices were published in [Ger08c, Ger08a]. 


\subsection{Conclusions}

Summary Realization of MOVPE grown SK-QD and SML based VECSEL devices evidenced clear advantages of QD-based gain media for this type of laser devices. Employing the spectrally broad gain of highly optimized, stacked SK-QD layers solved one of the biggest challenges of the VECSEL concept, the temperature sensitivity. Spectral QD gain matching with the semiconductor gain-chip resonance was assured for a wide temperature range. Maximum achieved device $\mathrm{CW}$ output power of $\approx 0.3 \mathrm{~W}$ at $1210 \mathrm{~nm}$ was already suitable for frequency up-conversion, as demonstrated by a similar QW-based device [Här07].

An overview of all realized VECSELs is given in Table 6.1, depicting device design and performance characteristics. Up to $1.4 \mathrm{~W} \mathrm{CW}$ output at $1034 \mathrm{~nm}$ and $12.4 \%$ differential efficiency is realized with the SML concept. Linearly designed QD-VECSEL showed reduced efficiencies, which are partly attributed to a non-optimal setup, including an OC with too high reflectivity. Nevertheless, ultra-low threshold of only $2.4 \mathrm{~kW} / \mathrm{cm}^{2}$ is achieved at $1210 \mathrm{~nm}$ wavelength by GS emission of highly optimized and stacked SK-QD active layers, and maximum CW output power of $300 \mathrm{~mW}$ equals the previously reported ES QD-VECSEL performance at $1040 \mathrm{~nm}$ wavelength.

Key design and growth parameters of this research are:

- Spectrally broad QD gain allows for a cavity resonance defined emission wavelength eliminating the conceptual strong VECSEL temperature sensitivity.

- MOVPE processes for stacks of up to 30 QD layers within a low-loss design were developed. Thus complete pump light absorption, large gain, and minimized losses enable reliable GS QD-VECSEL operation beyond wavelength of $1.2 \mu \mathrm{m}$.

- First SML based VECSELs prove similar in performance to QW based devices.

- Lattice matched $I n G a P$ was introduced as an alternative window layer material eliminating the need for a pump light absorbing $A l G a A s$ barrier plus $G a A s$ oxidation cap combination.

- The accelerated device degradation mechanism was identified as decreasing DBR quality due to acceptor enhanced defect formation as a consequence of the strong carbon background doping of TBAs grown $A l G a A s$ layers.

A two-step growth process realizes growth economies with separate epitaxial runs for DBR and RPG structure growth, eliminating the need for time-consuming and cost-driven regrowth of multi-microns thick DBR structures for every new sample. 
Outlook Future devices need to focus on different aspects for optimum performance:

Efficient available pump light usage, gain optimization and pump power balancing, advanced processing and mounting for maximized thermal dissipation. Such design improvements will lead to even higher output power in the multi watt range. Advances towards higher output powers for QD-based VECSELs were recently reported through work by other groups [But09, Rau10, Alb11].

Pump light injection can be improved by usage of antireflection coating at the gainchip surface in order to maximize the total efficiency. Only a fraction of the incident pump light is absorbed within the RPG, and can consequently generate charge carriers. Pump power that has not been absorbed adds to the total heat generation and directly reduces device efficiency. The need for thick absorbing matrix layers can be reduced by employing double stop band DBR designs that reflect the emission as well as the pump light wavelength [Här01, Ger03]. Such double stop band DBR designs additionally improve pump homogeneity within the active layer stack. Thus pump power balancing is supported, which aims at evenly distributed charge carriers to all active layers. Further pump balancing can be achieved by pump power dividing barrier layers, and/or a non-linear distribution of active layers as employed for the initial design. Maximum gain is achieved by an optimal overlap of the optical standing wave with the active layers. To improve this optical confinement-factor, close stacking by strain compensation is possible. Additionally, field strength within the RPG sub-cavity can be increased by adding DBR layers on the window side of the RPG structure. Besides the transparent intra-cavity heat spreaders as employed in this work, thermal energy dissipation can be improved by advanced processing, which not only involves wafer thinning but complete substrate liftoff. This can further improve VECSEL characteristics as performance of devices relies strongly on the optical quality of such intra-cavity elements [Kim06, Kim07]. Use of QD based VECSEL could enable a wider spectral tuning range as that of complex multi-chip setups based on QWs [Fan07a]. 


\section{Chapter 7}

\section{Electro-optically modulated vertical-cavity surface-emitting lasers}

Modern day fast growing data communications rely on optical links via fiber optic cables [Cam06]. Growth of the worldwide internet protocol (IP) traffic will proceed at a compound annual growth rate (CAGR) of $34 \%$, and is expected to quadruple from 2009 to 2014, as shown in Figure 7.1. This growth is mainly driven by consumers, which account for $87 \%$ of the total IP data volume and outpace the total IP traffic growth rate by $36 \%$ CAGR.

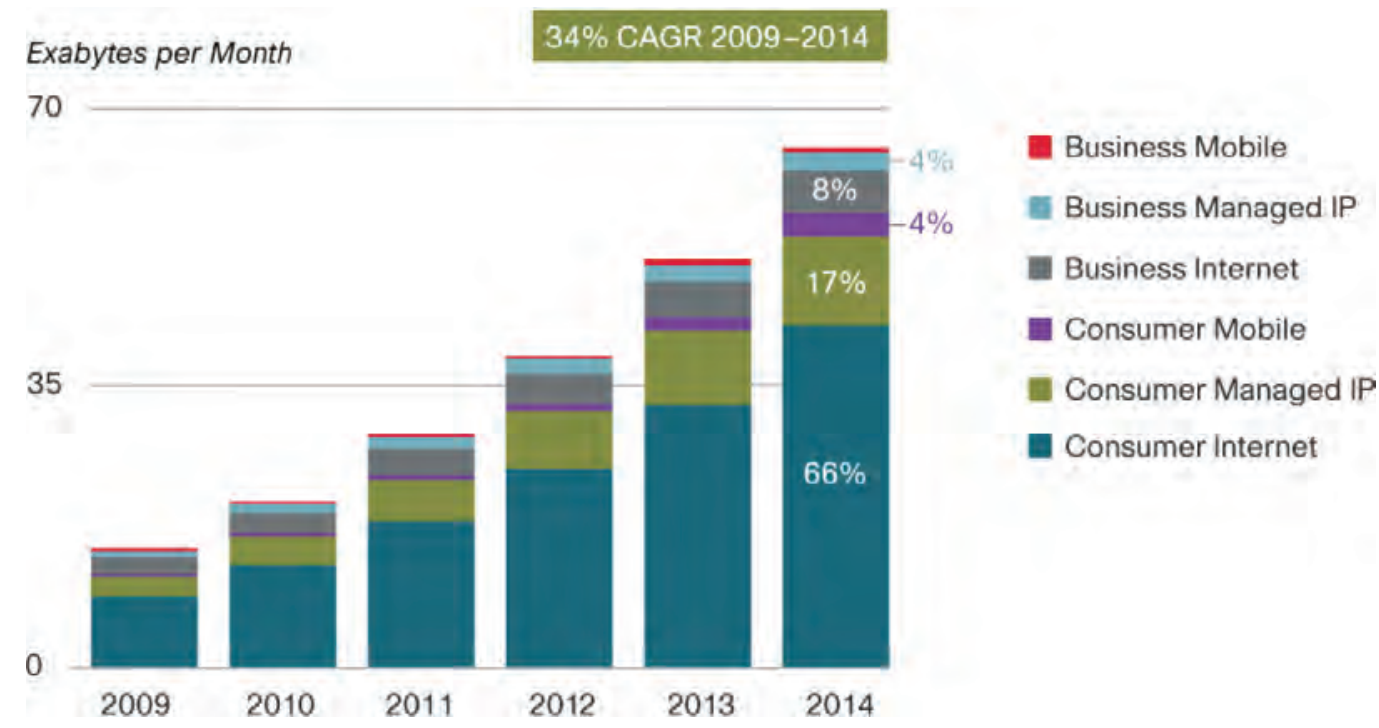

Figure 7.1: Diagram shows the projected growth of the global IP traffic. Consumer demand is overwhelmingly driving the global IP data volume. Source: Cisco Visual Networking Index, 2010, "Hyperconnectivity and the Approaching Zettabyte Era".

Lasers with external modulators are used for long-distance connections to send signals at the highest data rates, in the range of $40 \mathrm{Gbit} / \mathrm{s}$. Such optical transmitter units usually consume some tens of watts of electric power and are not suitable for the highly-integrated and low-cost consumer products which are on the verge of reaching everybody's homes. For shorter optical interconnects with moderate data rates in the range of 5 to $10 \mathrm{Gbit} / \mathrm{s}$, direct-modulated VCSELs are used. On the research level, repetition rates of up to 20 GHz have been reached using direct modulation [Blo09], but these devices are not yet 
commercially available. Further speed increases prove to be demanding due to the fact that the modulation speed increase of conventional current-modulated VCSELs requires a roughly quadratic increase in the VCSEL current density $j$ for linear repetition rate $f_{r}$ increase.

$$
f_{r}=\frac{1}{2 \pi} \sqrt{\frac{1}{\tau_{n} \tau_{p h}} \frac{j-j_{t h}}{j_{t h}-j_{0}}}
$$

This conventional approach to higher repetition frequencies can lead to accelerated device degradation caused by the very high but necessary drive currents [Wes09]. In this work a monolithically integrated concept was investigated to overcome the need for separate modulators at highest speeds while maintaining the established VCSEL technology.

The presented integrated concept was realized by employing an electro-optic modulator (EOM) based on the quantum confined Stark effect (QCSE) integrated into a VCSEL structure. The VCSEL section is driven in CW mode, while the EOM section is integrated into the top DBR to change the out-coupling efficiency of this compound-cavity laser system. Simulations and concept design for this device were first presented by Shchukin et al. [Shc08]. The whole EOM VCSEL device can be grown by a single epitaxial growth run and employs the same established processing and mounting technologies as conventional current-modulated VCSELs. In consequence, this novel monolithic EOM VCSEL approach aims at low-cost but ultra-fast transmitter components suitable for consumer applications.

\subsection{Electro-optical modulator}

The goal of an EOM is to effectively change the optical characteristics of a medium such as the refractive index using an externally applied electric field. External optical modulation by an EOM concept was already demonstrated in 1988 using a relatively thick external modulator medium with an 82-fold GaAs QW stack [Lee88]. To create a monolithic device, the EOM needs to be shrunken to fit into a VCSEL design while maintaining its modulating capability.

\subsubsection{Quantum confined Stark effect}

Analogous to the conventional Stark effect, which describes the influence of an electric field on the emission lines of atoms and molecules, the QCSE explains the influence of an electric field on quantum confined charge carriers. This quantum confinement is realized for carriers in a semiconductor QW system. In contrast to an electric field applied to bulk semiconductors, which basically gives a broadening of the band-edge absorption explained by the Franz-Keldysh effect, the QCSE requires quantum confinement of charge carriers within semiconductor nanostructures. This QCSE was first proposed and studied 

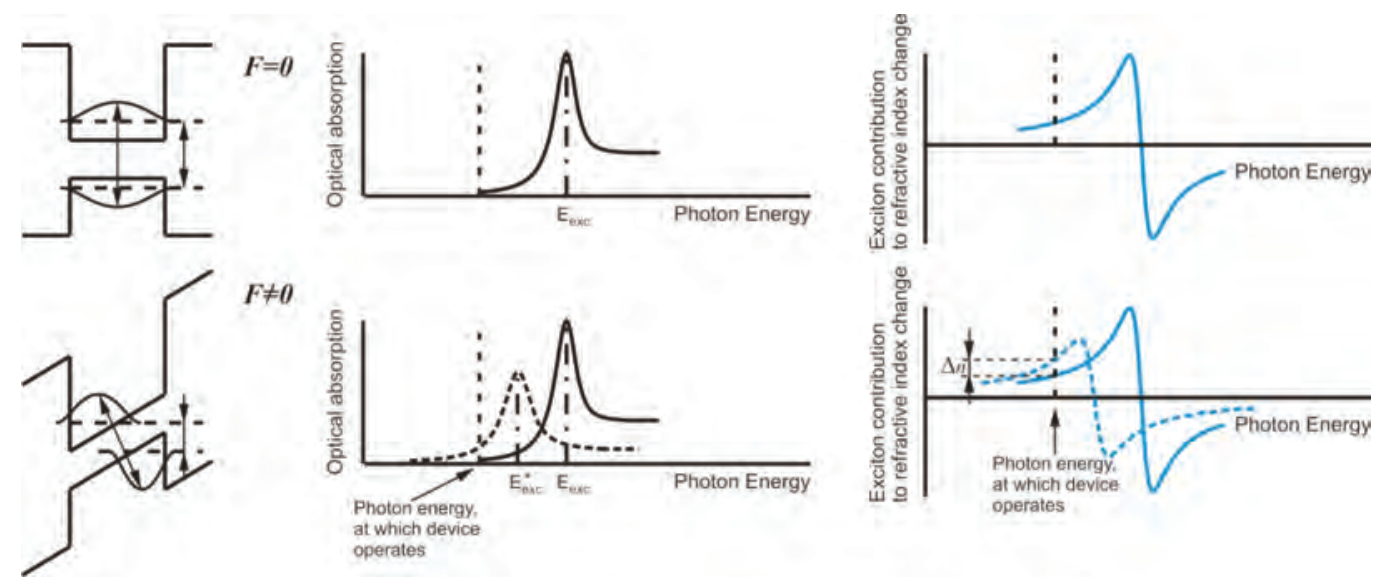

Figure 7.2: Schematic depiction of the influence of an applied field $F$ on the refractive index change $\Delta n$ via the QCSE on QW structures based on presentation of [Shc08].

by Miller et al. [Mil84, Mil85]. They observed broadening and large red shifts of the exciton absorption peak of QWs in an electric field perpendicular to the semiconductor layers. This reduction of the exciton energy is caused by the tilt of the band structure due to the applied field. In consequence, the energetic distance between the bound QW hole and electron states is lowered, as shown in Figure 7.2. As the excitonic peak shifts, the absorption for a distinct spectral position is altered, and by Kramers-Kronig relation the effective refractive index is changed as well. The presented device uses this refractive index change to alter the out-coupling efficiency of the VCSEL section.

\subsubsection{Modulator design}

The efficient design of an EOM aims at a maximized refractive index change by a minimal applied electrical field change $d n / d E$. In parallel, the design needs to avoid absorption within the EOM section. Lee et al. already identified two operating regimes for their external EOM: first, a narrow spectral region adjacent to the exciton peak exhibiting large refractive index and large absorption changes; and second, a broader spectral region at a 10 to $25 \mathrm{~nm}$ longer wavelength with respect to the absorption peak [Lee88]. The first system shows the more important effects, but optical modulation would also be based largely on absorptive effects, while for the second region dispersive effects can be dominant and a high transparency for the lasing wavelength is achievable.

The presented design focuses on the second regime, thus the lasing wavelength is tuned to an about $15 \mathrm{~nm}$ longer wavelength when compared to the modulator QWs within the EOM section. Investigated test EOM samples showed a maximum refractive index change of $\Delta n \approx 0.01$ employing a five-fold $G a A s / A l_{0.2} G a_{0.8} A s$ QW stack. This multi quantum well (MQW) modulator is placed in a second cavity within the top DBR of the VCSEL 
Figure 7.3: Modulation principle of the EOM VCSEL: The exciton absorption peak is shifted due to the QCSE by applying an electric field; In consequence, the refractive index is altered too; The EOM cavity resonance dip can be shifted out of resonance with the VCSEL cavity; Compared to the resonance position the light transmission is significantly changed. Figure from presentation of ref. [Shc08].

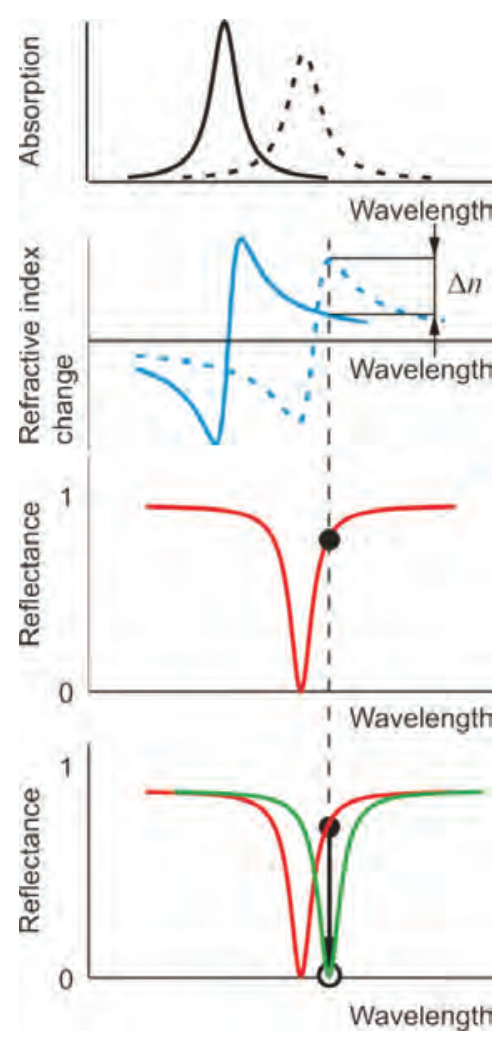

structure to enhance its effect. In consequence, the refractive index change caused by the QCSE shifts the resonance dip of the cavity, and thus modulates the out-coupling efficiency of the laser. The principle of the concept is shown in Figure 7.3. Inserting the EOM part into a cavity results in enhanced optical field strength at the position of the modulator MQW and consequently enhances the modulating strength.

\subsection{Monolithically integrated EOM VCSEL design}

Design considerations for this EOM VCSEL prototype aimed at a fully operational proofof-principle device at the relevant short-range optical-interconnect wavelength of $945 \mathrm{~nm}$. The design includes three DBR parts enclosing two cavities. A 37.5-fold bottom n-DBR and middle p-DBR form the active VCSEL cavity; a middle p-DBR and top 10-fold $\mathrm{n}$-DBR enclose the EOM cavity. The middle p-DBR consists of $30.5+8$-pairs separated by a lattice matched $\operatorname{InGaP}$ etchant-stop layer adjacent to a $G a A s: p^{+}$contact layer placed within a $\lambda / 4$ layer of this DBR. This layer combination was subsequently used to define a metallic middle contact separating EOM and VCSEL sections of the device. All other DBR parts defining both cavities consist of $A l_{0.9} G a_{0.1} A s$ and $A l_{0.15} G a_{0.85} A s$ $\lambda / 4$ layers employing linearly graded interfaces. These graded layers were grown with doubled doping levels compared to the fixed composition layers to facilitate charge carrier 

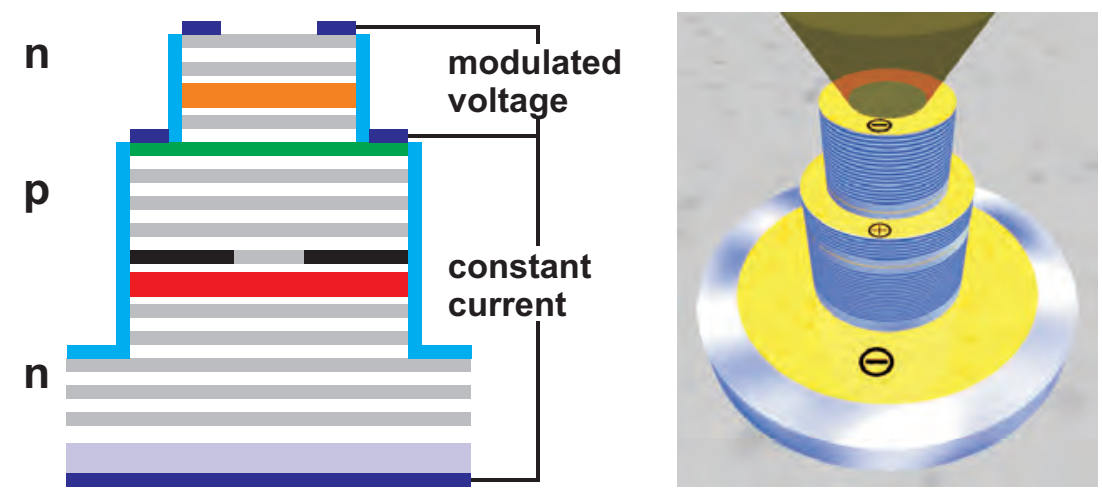

Figure 7.4: Figure shows the schematic cross-section and a drawing of the processed EOM VCSELs. The larger VCSEL section at the bottom is operated in the CW mode while modulated voltage is applied to the top EOM section to modulate the light output.

transport at the numerous interfaces. Doping of the DBRs was performed with $\mathrm{SiH}_{4}$ and $D M Z n$, as detailed in 4.2. A conventional one- $\lambda$ cavity design with a four-fold MQW GaAs $/ A l_{0.2} G a_{0.8} A s$ gain-medium was chosen for the VCSEL section. For the target lasing wavelength of $845 \mathrm{~nm}$ the active QW-emission was set to $\approx 835 \mathrm{~nm}$ at RT to account for device heating upon operation. Adjacent to the active cavity, an aluminum-oxide current-aperture was formed by post-growth oxidation of $A l G a A s$-gradings bordering an $A l_{0.98} G a_{0.02} A s$ layer. This aperture layer was included within the first middle DBR period. The modulator cavity was based on the same layer structure but included a five-fold MQW GaAs/ $A l_{0.2} \mathrm{Ga}_{0.8} \mathrm{As}$ EOM-medium with the GS transition at higher energy to avoid absorption. A schematic of the complete device structure is given in Figure 7.4 .

To ensure automatic matching of both cavities within the device, both cavities were designed to have nominally the exact same optical length. This was realized for the fourfold MQW VCSEL cavity and the five-fold MQW EOM cavity by adapting QW and barrier thicknesses to form an equal optical length in total. The complete structure totals to about 400 layers and is very demanding in terms of growth accuracy and homogeneity.

\subsubsection{Calibrations and growth}

Epitaxial growth of this demanding EOM VCSEL design required an extensive amount of calibration and fine tuning samples to align both cavities and DBR properties, as well as to set a large variety of precise material compositions, all combined with a multitude of different doping levels. All parts of the EOM VCSEL were calibrated individually by specially designed test structures e.g. a single cavity within a reduced number of DBR layers to set the resonance dip position within the DBR stop band range. PL investigations for the optically active test samples and XRD measurements for lattice matching and compositional settings were carried out. Doping levels for n- and p-doped layers were 


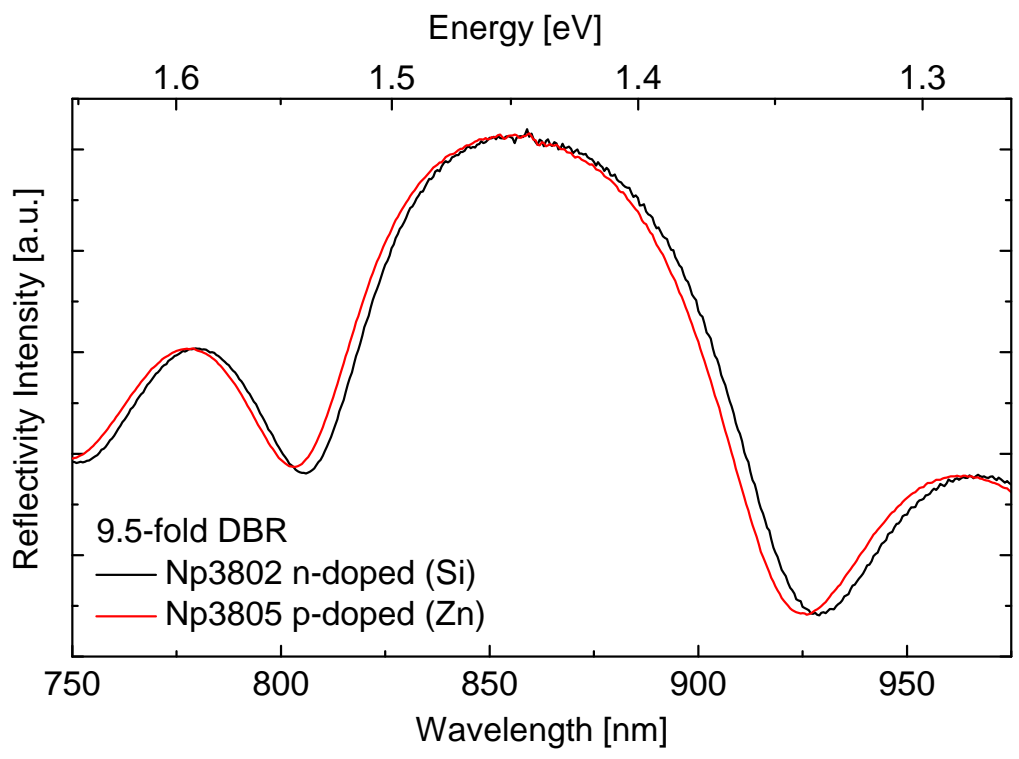

Figure 7.5: Reflectivity of two nominally identical 9.5-fold DBR test structures. The sole difference is doping. They are either p-doped with $\mathrm{Zn}$, or n-doped with Si. Spectral difference of the stop-band position is measured to $3 \mathrm{~nm}$.

tuned to values between $5 \cdot 10^{17}$ to $4 \cdot 10^{18}$ and verified by Hall measurements. All layers could be tuned within this range except for the $\mathrm{Al}_{0.9} G a_{0.1} \mathrm{As}: \mathrm{Si}$ which maxes out around $n=7 \cdot 10^{17}$ due to the self-compensating behavior of the group IV element silicon. Transfer-matrix method simulations were employed to analyze surface reflectivity measurements of DBR and cavity test samples. For the simulation, an intermediate layer with an averaged constant composition approximated graded layers with good accuracy.

Reflectivity measurements show that growth rates were altered depending on the employed dopant source. Thus stop band positions of the p-DBR and n-DBR varied for identical growth parameters and needed to be tuned separately to the same spectral position (cf. Figure 7.5). In consequence, the resonance dips of the n-i-p VCSEL cavity with adjacent aperture layer and the p-i-n EOM cavity in proximity to the InGaP/GaAs: $p^{+}$ etch-stop/contact layer did not show the intended automatic matching and were tuned separately to the same spectral position. Figure 7.6 part (a) shows the very good accuracy of less than one nanometer of the spectral resonance dip positioning of EOM (red) and VCSEL (green) cavity resonances. Based on these test samples the final EOM VCSEL structure (black) was grown showing an overall resonance in between the separately calibrated positions. EOM and VCSEL cavity test samples were simulated separately and layer-model parameters were tuned to match dip positions to investigate run-to-run growth stability, cf. 7.6 part (b). Subsequently, the whole EOM VCSEL layer assembly was simulated employing the very same, unchanged layer-model parameters as for the test 


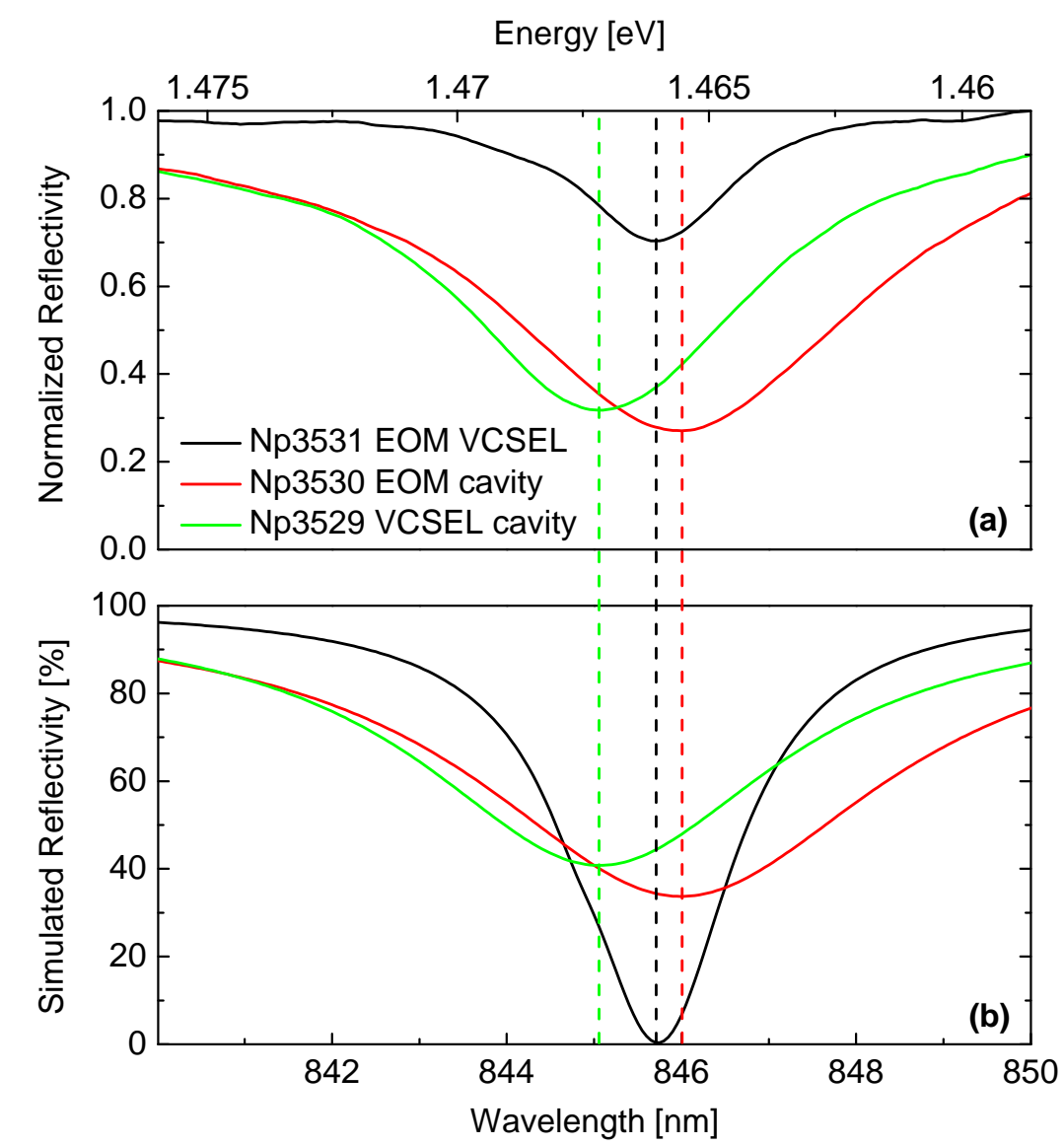

Figure 7.6: (a) Reflectivity measurements show the resonance dip positions of EOM and VCSEL cavity test samples. Spectral positioning accuracy of both cavities is achieved within $1 \mathrm{~nm}$. The resonance dip of the final complete EOM VCSEL structure based on the shown calibration samples is positioned in between the test sample resonances.

(b) Transfer-matrix simulations of the test samples are tuned to the measured resonance-dip positions. Layer-model parameters of these test sample simulations are used without changes to simulate the whole EOM VCSEL structure. This complete layer model shows perfect matching of the simulated dip position with the measured value, without any additional model parameter modifications.

dashed lines: Guides for the eye are shown at the measured dip positions.

sample simulations. Comparison of the measured and the simulated EOM VCSEL dip positions illustrates a perfect spectral match. The sole difference was based on the depth of the resonance dip, which was attributed to the simplified model employing a constant imaginary part value of the refractive index for all layers with band edge energies beyond the calculated wavelength. These results demonstrate the excellent predictability and accuracy of the MOVPE growth technology, also for high precision growth runs with several 


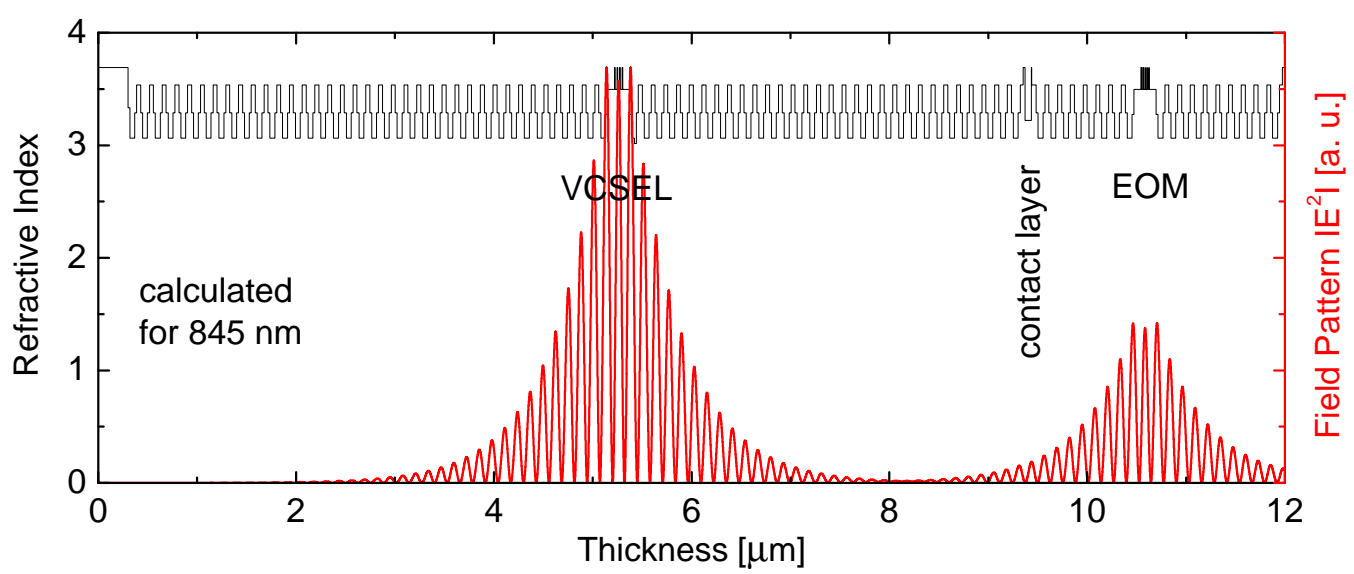

Figure 7.7: black: Refractive index pattern of the grown structure. Graded DBR interfaces are approximated for simulation by intermediate layers with constant refractive index levels. Positions of the VCSEL cavity, EOM cavity, and contact layer are indicated.

red: Optical field-intensity wavepattern of the VCSEL cavity resonance wavelength. Simulation is based on the transfer matrix method with a constant absorption approximation included for all layers beyond the band edge of the calculated wavelength. Nanostructure effects are not included.

hundreds of layers, and evidences the accuracy of the optimization process based on small, individually optimized test structures in combination with transfer matrix simulations.

Optical field-intensity wave-pattern was simulated with the aforementioned transfer matrix method to verify the overall design. The result for $845 \mathrm{~nm}$ is shown in combination with the refractive index pattern of the whole grown structure in Figure 7.7 . Simulation was carried out without including gain parameters. Maximum field intensity was predicted for the VCSEL cavity, while significant field strength was observed in the EOM part, enabling output power modulation.

The final device was grown by a single arsine-based MOVPE growth run at $700^{\circ} \mathrm{C}$ for the entire monolithic EOM VCSEL structure. Growth temperature was lowered to $615^{\circ} \mathrm{C}$ only for the deposition of the lattice matched In $G a P$ etchant-stop layer, employing the previously described switching procedure (cf. Table 4.5). After switching back from TBP to arsine, the $I n G a P$ layer was covered with $5 \mathrm{~nm} \mathrm{GaAs}$ prior to heating up again to resume DBR growth. Growth was finalized by a GaAs $: n^{+}$contact layer serving as oxidation protection. Epitaxy on 2 inch $G a A s$ : Si ( $\left.\begin{array}{lll}1 & 0 & 0\end{array}\right)$ substrates yielded good uniformity across the whole wafer and a smooth surface quality.

\subsubsection{Processing and characterization}

Standard lithography and dry etching techniques were employed to process the EOM VCSELs with varying double-mesa diameters from 25 to $36 \mu \mathrm{m}$ and 45 to $56 \mu \mathrm{m}$ for EOM and VCSEL sections, respectively. All processing for this device was performed by my 


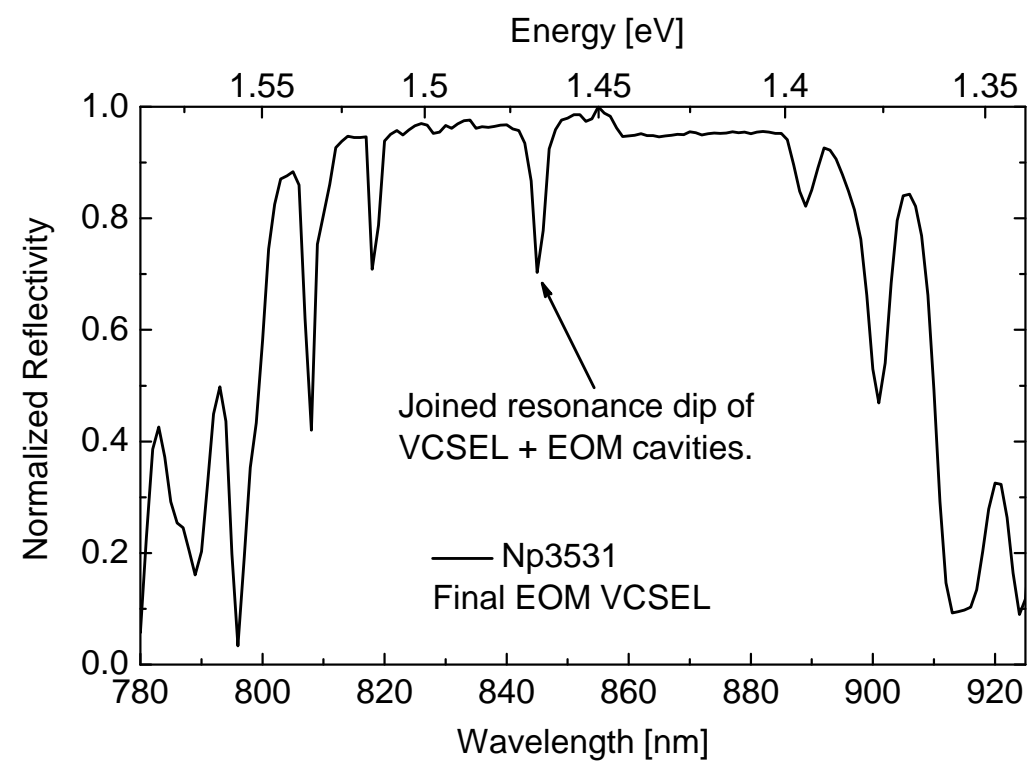

Figure 7.8: Surface reflectivity measurement of the unprocessed EOM VCSEL wafer is shown. The joined EOM + VCSEL resonance dip is precisely positioned within the DBR stop band. FWHM of the dip is less than $2 \mathrm{~nm}$.

former colleague Alex Mutig as described in [Hop07]. Selective oxidation of the Al-rich aperture layer was done using optimized conditions as described in ref. [Hai02]. Upon oxidation, the volume of the aperture layer is reduced between $6.7 \%$ and $13 \%$, depending on the aluminum content [Cho97]. Consequently, the design needed to deal with significant strain at the interfaces to the adjacent $G a A s$ layers. To avoid interlayer delamination or fracture, graded $A l G a A s$ layers were grown enclosing the aperture layer. Three ohmic contacts were formed for device operation, bottom and middle contact for the VCSEL part, middle and top contact for the EOM part. The bottom contact is formed on the underside of the thinned substrate, while middle and top contacts are circular contacts on the respective mesas (cf. Figure 7.4).

For device operation, the EOM VCSELs were mounted onto a copper heat sink and operated at RT as detailed in [Blo09]. In parallel to the optical output power of the laser, the electric photocurrent generated within the EOM section was recorded. This measured current was due to unintended photo absorption of the lasing wavelength.

\subsection{EOM VCSEL results}

Initial characterization prior to processing included surface microscopy and reflectivity measurements. Surface reflectivity is given in Figure 7.8 , evidencing the precise positioning of the joined resonance dip of VCSEL plus EOM cavity within the DBR stop band. The 


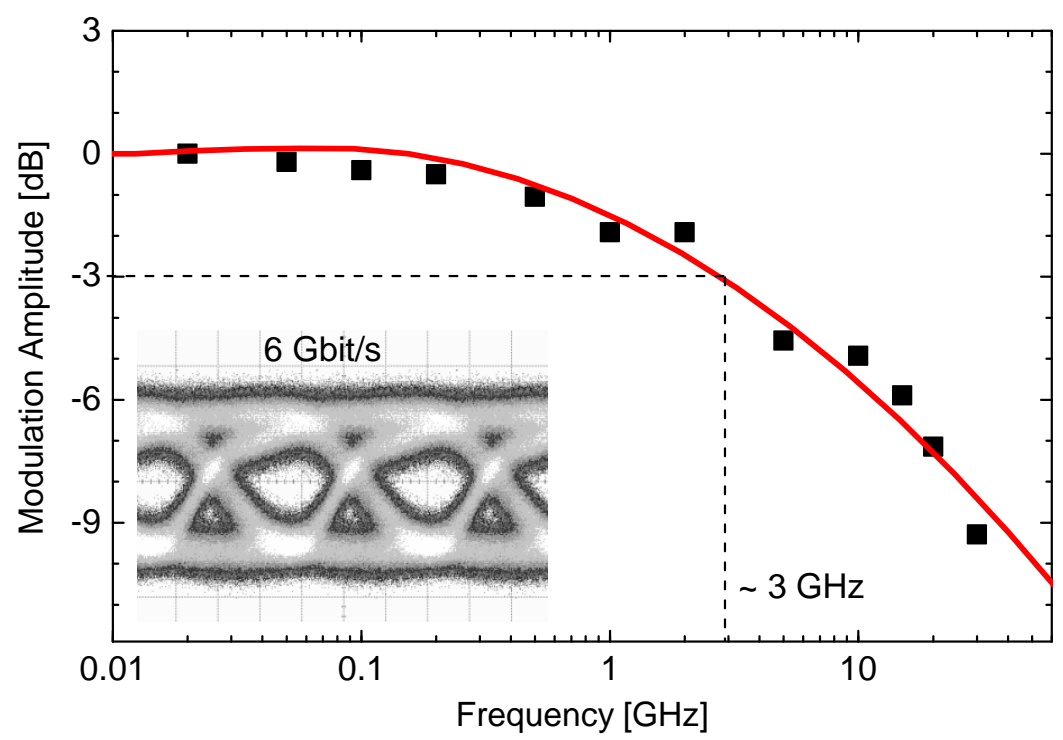

Figure 7.9: black squares: RT small signal modulation measurement shows an optical bandwidth of about $3 \mathrm{GHz}$ for a device with $25 \mu \mathrm{m}$ EOM-mesa diameter.

red line: Fit curve showing a $3 \mathrm{~dB}$ bandwidth of $\approx 3 \mathrm{GHz}$.

inset: Eye diagram at $6 \mathrm{Gbit} / \mathrm{s}$, eyes are fairly open up to $10 \mathrm{Gbit} / \mathrm{s}$, average output power is $\approx 1$ $\mathrm{mW}$ at $1 \mathrm{~V}$ modulation voltage applied to the EOM section.

shape of the dip is without any significant tendencies to splitting, proving the precise matching of both cavities.

\subsubsection{High speed operation}

As the device is targeted for high-speed operations, the optical bandwidth of the EOM VCSEL is an important factor for applications. Thus, initial characterization focused on dynamic modulation behaviors of the novel device design. All dynamic measurements were carried out with constant current applied to the VCSEL section, while modulation was solely done via EOM voltage variation. Small signal modulation experiments showed a $3 \mathrm{~dB}$ bandwidth of $\approx 3 \mathrm{GHz}$. Open $6 \mathrm{Gbit} / \mathrm{s}$ eye diagrams at RT were measured with an EOM modulation voltage of $1 \mathrm{~V}$, and yielding an average output power of $\approx 1 \mathrm{~mW}$ (cf. Figure 7.9). These dynamic characteristics of the prototype EOM VCSEL device were lower than expected for the EOM concept. The bandwidth limitation is attributed to electrical parasitic effects, especially within the EOM section, which includes no oxide aperture and additionally suffers from increased absorption of the laser wavelength by the EOM QWs upon modulation. The electro-optic effect itself is fast but relies on the field strength at the EOM QW stack. Photo generated carriers within the EOM cavity will counteract the applied field and limit modulation properties. 


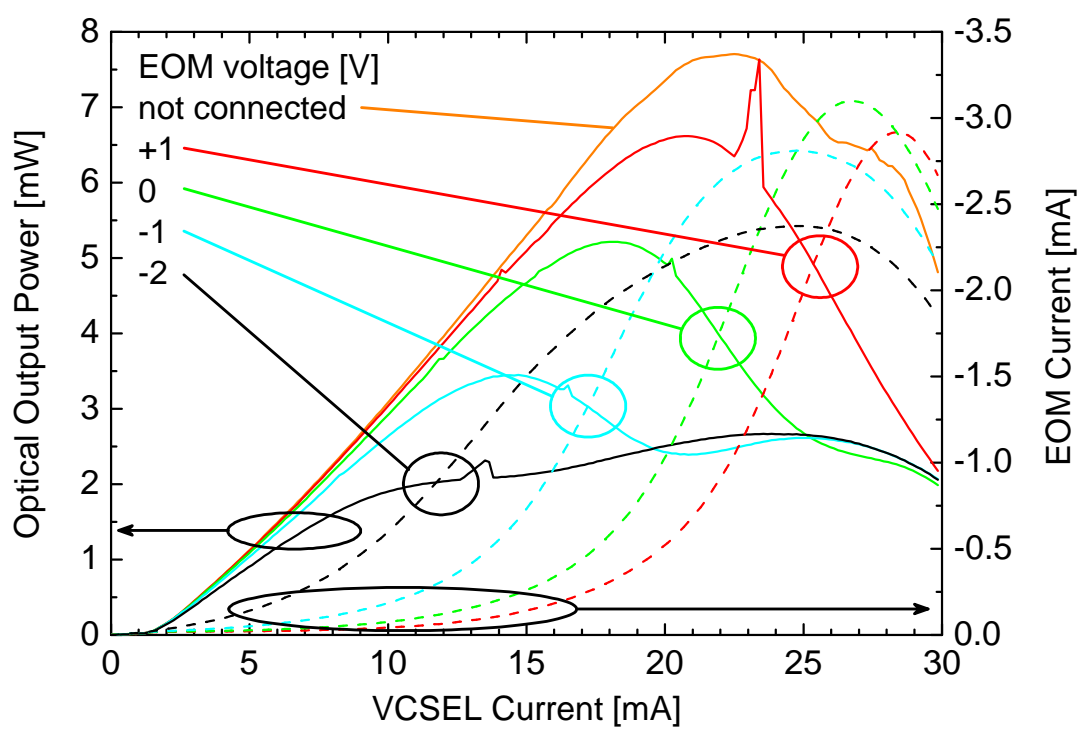

Figure 7.10: solid lines: P-I curves at varied EOM voltages show the static EOM VCSEL characteristics of a device with $36 \mu \mathrm{m}$ EOM-mesa diameter. $7.7 \mathrm{~mW}$ maximum output power is achieved with disconnected EOM section. Modulation upon voltage change from the monolithically integrated EOM section is clearly evidenced.

dashed lines: Measured EOM photocurrent due to absorption within the modulator section is shown for comparison.

\subsubsection{Static modulation}

Static measurements were carried out to investigate the behaviors of the VCSEL and EOM sections. Figure 7.10 shows characteristic P-I graphs for a variety of reverse bias levels applied to the EOM section, dashed lines additionally indicate the corresponding photocurrents generated within the EOM section. This photocurrent was increased at higher VCSEL drive currents, indicating enhanced absorption of the lasing wavelength within the modulator section. Highest optical output power of up to $7.7 \mathrm{~mW}$ was achieved with disconnected EOM section. This maximum output is attributed to transparency of the EOM section by saturation with photo-generated charge-carriers. A modulation amplitude of the optical output of more than $3 \mathrm{~dB}$ was achieved for a change of the EOM reverse bias from $0 \mathrm{~V}$ to $-2 \mathrm{~V}$. Simultaneously the generated photocurrent increased, indicating enhanced absorption within the EOM section. Thus, the modulation is at least partially based on absorption modulation, which is not intended for this type of device. A quantitative analysis of the modulation mechanisms is given in the following Section 7.3.3. The spikes visible in all P-I graphs and shifting with EOM voltage to higher VCSEL currents indicate some special working points which were investigated in more detail in Subsection 7.3.4. 
Figure 7.11: Temperature influence on the modulation behavior is shown for $25^{\circ} \mathrm{C}$ and $85^{\circ} \mathrm{C}$ operation.

solid lines: P-I-curves at $0 \mathrm{~V}$ and $-2 \mathrm{~V}$ EOM voltage show significant modulation for both temperatures.

dashed lines: Extinction ratio is calculated for both temperatures demonstrating improved modulation amplitude at higher temperature.

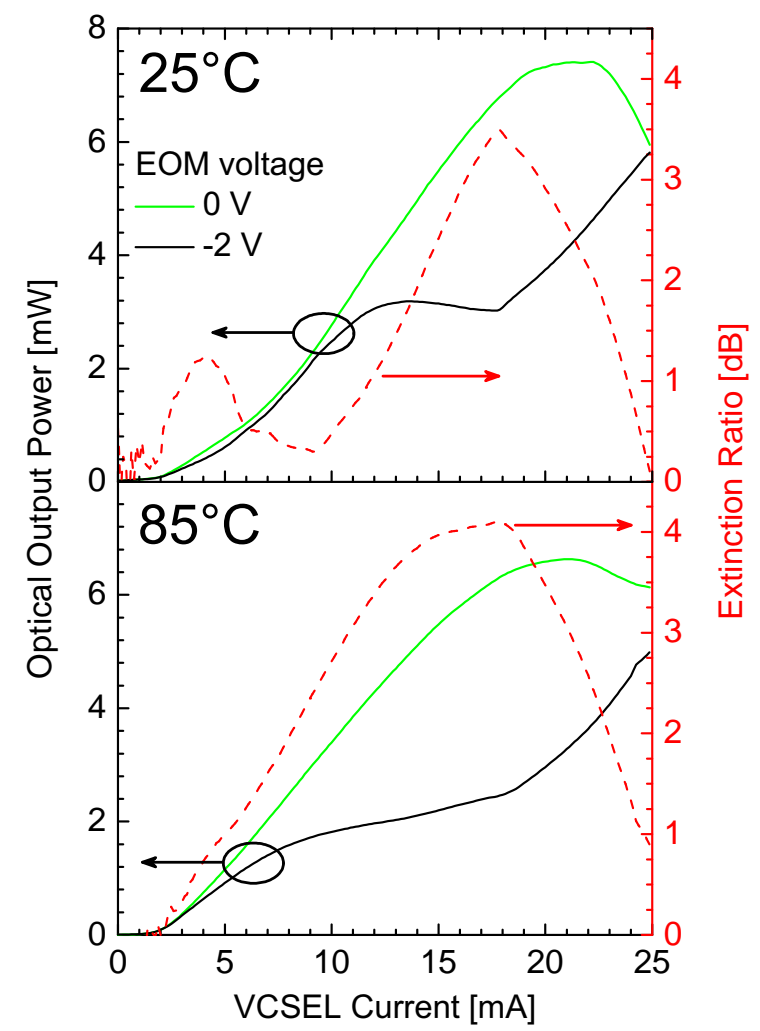

As thermal stability is crucial for real world commercial devices, one should allow for operating temperatures as high as $85^{\circ} \mathrm{C}$. Modulation behavior of the EOM VCSELs at RT and high temperature is shown in Figure 7.11. Measurements demonstrate that the 3 $\mathrm{dB}$ extinction ratio at $\mathrm{RT}$ was slightly improved for operation at $85^{\circ} \mathrm{C}$ to $\approx 4 \mathrm{~dB}$ and was accessible over a wider drive current range of the VCSEL section. This finding is probably due to an improved matching of both cavities at the elevated device temperature. At both temperatures the maximum extinction ratio was achieved around $16 \mathrm{~mA}$ VCSEL drive current, indicating a potential working point for modulation.

\subsubsection{Limitation analysis}

The ratio of electro-optic effect-based and absorption-based modulation within the EOM section was quantified in order to investigate the optical bandwidth limitations. Part (a) of Figure 7.12 shows P-I graphs and photocurrent curves of Figure 7.10 around the main modulation working point at $16 \mathrm{~mA}$ VCSEL current. The $0 \mathrm{~V}$ data (green) was taken as reference state while the $-2 \mathrm{~V}$ EOM voltage data (black) was interpreted as modulated state. In part (b) the difference between both states was calculated for photocurrent and output-power data. The dashed line represents the measured EOM photocurrent change $\Delta I_{E O M}$, while the solid line is the change of the optical output power $\Delta P$ converted to 


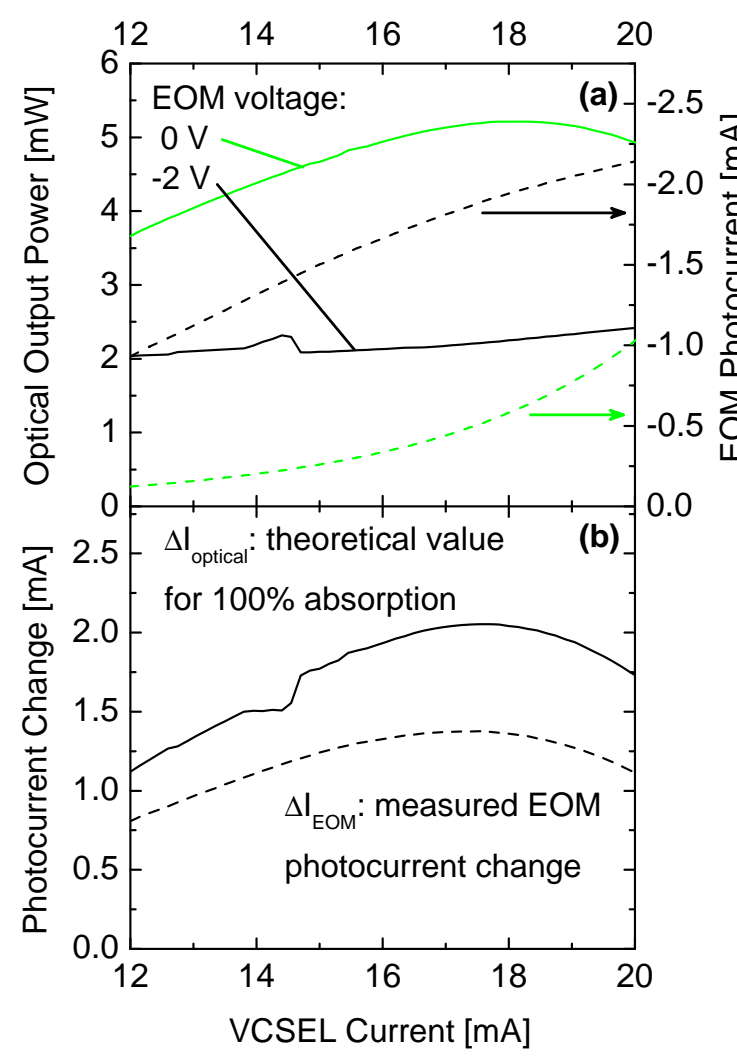

Figure 7.12: (a) Part of the data of Figure 7.10 is shown around a possible working point position with maximum extinction ratio.

(b) Differences of the data in (a) are calculated to plot the EOM photocurrent change $\Delta I_{E O M}$ and the theoretical maximum photocurrent change for $100 \% \mathrm{ab}$ sorption $\Delta I_{\text {optical }}(\lambda)$.

solid lines: Data is based on measurements of the optical output power.

dashed lines: Data is based on the measured EOM photocurrent due to absorption within the modulator section.

the corresponding theoretical maximum photocurrent difference $\Delta I_{\text {optical }}(\lambda)$ according to equation 7.2

$$
\Delta I_{\text {optical }}(\lambda)=\Delta P \frac{\lambda}{h c} e
$$

Absorption-based modulation is represented by $\Delta I_{E O M}$, while $\Delta I_{\text {optical }}(\lambda)$ corresponds to the total modulation amplitude of the optical output. Thus $\Delta I_{\text {optical }}(\lambda)$ can be taken as an equivalent to a theoretical $100 \%$ absorption-based modulation at wavelength $\lambda$. By calculating the ratio of both curves in part (b) of Figure 7.12 the percentage of the total modulation based on the electro-optic effect can be plotted. This percentage is plotted for different EOM voltages in Figure 7.13 A reverse bias of $-2 \mathrm{~V}$ applied to the EOM section shows an almost flat, VCSEL drive current-independent behavior with close to $30 \%$ electro-optic effect-based modulation. Higher EOM reverse voltages showed an increasing electro-optic effect-based modulation for higher VCSEL drive currents. This analysis shows that modulation for most working points is predominantly based on absorption. Thus, the EOM section acts mainly as an externally triggered absorption modulator. However, a relevant fraction of the modulation amplitude can already be attributed to the intended electro-optic effect-based modulation. Consequently, the relatively high field-strength change of $\approx 87 \mathrm{kV} / \mathrm{cm}$ (calculated for $-2 \mathrm{~V}$ ) required to achieve a $3 \mathrm{~dB}$ modulation depth is 
Figure 7.13: Percentage of the total modulation amplitude (including absorption) which is attributed to the electro-optic effect. Data is calculated from part (b) of Figure 7.12 for $-2 \mathrm{~V}$ and from equivalent data for $-3 \mathrm{~V}$ and $-4 \mathrm{~V}$ EOM voltage. For higher reverse bias, EOM voltage percentage values increase with increasing VCSEL drive currents.

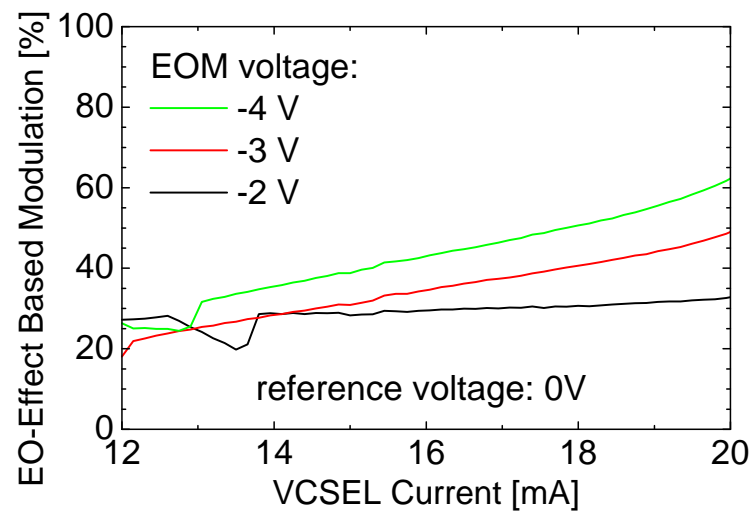

mainly attributed to the need to shift the QW absorption peak in and out of absorption, as comparable field-strength values have been reported to enable the required energetic shift of a $G a A s$ QW [Len90]. As the ratio of absorption-driven modulation to electro-optic effect-based depends on VCSEL drive current, efficient dynamic operation of an EOM VCSEL relies on the chosen working point as well as on the ability of future device designs to avoid EOM absorption, and thus parasitic effects within the modulator.

\subsubsection{Resonant electro-optic modulation}

Small spikes in the optical output power curves were already observed in Figure 7.10. Such unconventional P-I curve features were not only observed for this rather large device with an EOM-mesa diameter of $36 \mu \mathrm{m}$, but also for other device sizes. Figure 7.14 shows more detailed P-I data measured with a shorter step width from a device with smaller $28 \mu \mathrm{m}$ EOM-mesa diameter. Sharp maxima occurred for all applied EOM voltages and shifted for this device size to higher VCSEL drive currents with increased EOM reverse biases. This strong and very sharp second maximum is attributed to the fulfillment of the resonance condition of this compound cavity system of the EOM VCSEL device. This data demonstrates the significance of resonant coupling to efficiently change device output power. Investigations of our devices show that these resonance spikes can shift with increased EOM voltages, either to higher or to lower VCSEL drive current values depending on mesa sizes. Shchukin et al. reported a similar but much broader resonance feature for a comparable MBE grown EOM VCSEL, which occurs before the first output maximum of the device is reached [Shc08]. Whether the resonance feature appears before or after the VCSEL maximum depends most likely on the offset between VCSEL and EOM resonance positions. For a compound cavity system consisting of a laser diode coupled to an external feedback mirror, a comparable resonance feature is reported by Lang et al. [Lan80]. However, due to the smaller total dimensions of the present EOM VCSEL coupled-cavity system, only a single resonance peak can be observed in contrast 


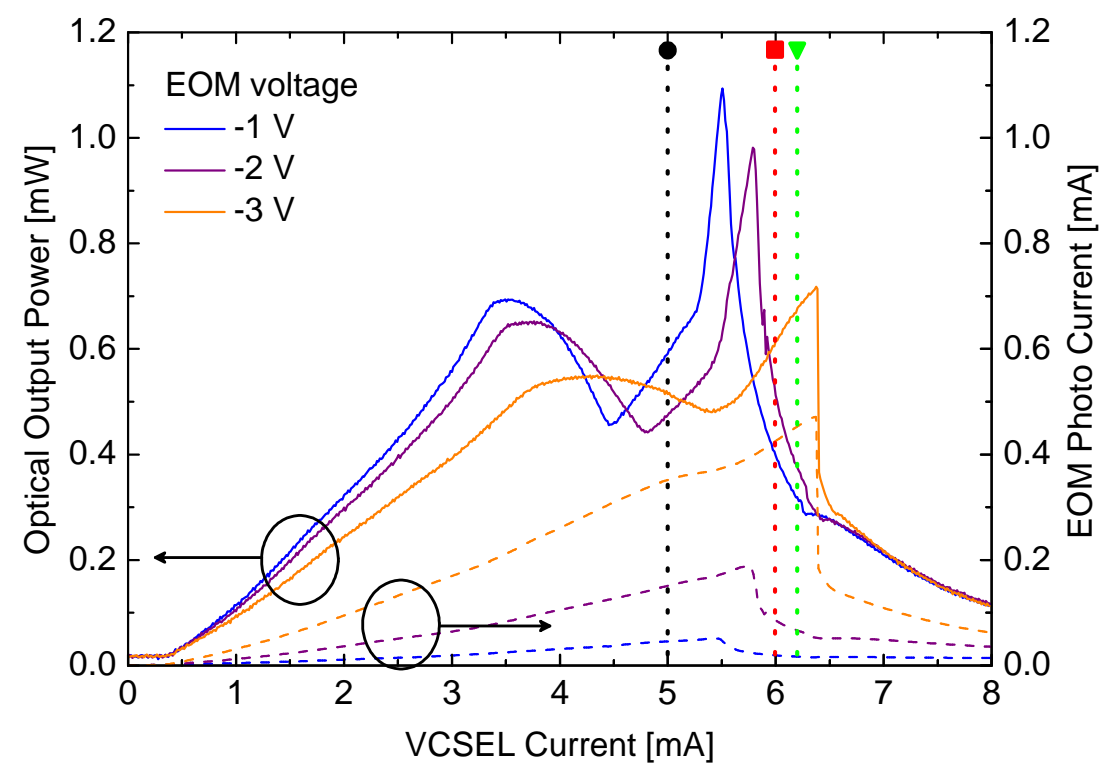

Figure 7.14: To identify the origin of the spikes observed in Figure 7.10 another device with smaller EOM-mesa diameter of $28 \mu \mathrm{m}$ was investigated by measurements with a smaller step width. For higher VCSEL drive currents above $5 \mathrm{~mA}$, sharp output power peaks are observed.

solid lines: Optical P-I characteristics with different voltages applied to the EOM section. dashed lines: Corresponding photocurrent due to absorption within the EOM section. dotted lines: Guides for the eye to allow identification of the position of data in Figure 7.15

to multiple amplitude undulations for larger changes in the reported external feedback system.

As shown in Figure 7.6 the VCSEL cavity resonance of our device was positioned at a slightly shorter wavelength than the EOM cavity resonance. With an increase in the VCSEL drive current, the VCSEL cavity shifts spectrally towards the resonance due to a growing operating temperature within the VCSEL section of the device. This resonance position depends on the applied EOM reverse bias, and shifts with an increase of the EOM reverse bias to lower (cf. Figure 7.10) or higher (cf. Figure 7.14) VCSEL drive currents, depending on whether the refractive index change $\Delta n$ has a positive or negative value upon modulation. The sign of $\Delta n$ depends on the offset between lasing wavelength and spectral absorption peak position of the modulating MQW section. As for our offset design, the EOM regime of region II described in Figure 1 of reference [Lee88] applies, both signs of $\Delta n$ are possible. This spectral laser-modulator offset depends on the temperature gradient between both cavities of the device, and can be altered by different device sizes, which alter the thermal properties. Consequently, the resonance position of this compound cavity system upon EOM modulation is moved to lower or higher VCSEL drive currents depending on device dimensions, geometry, and mounting. 


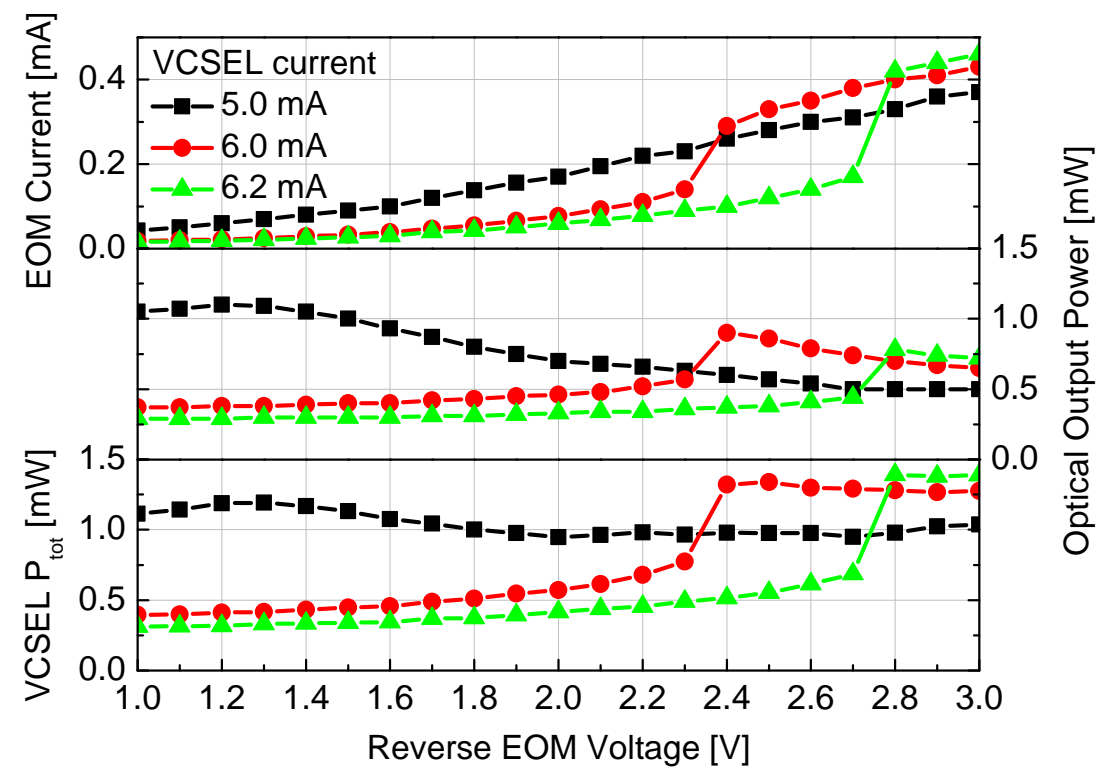

Figure 7.15: top: Measured EOM photocurrent. middle: Measured optical output power. bottom: Calculated total VCSEL section power loss $\mathrm{P}_{t o t}$ (EOM absorption + optical output). Curves for different drive currents of the VCSEL section are plotted. 5.0 mA shows a flat $\mathrm{P}_{\text {tot }}$ behavior, while $6.0 \mathrm{~mA}$ and $6.2 \mathrm{~mA}$ drive currents show distinct resonance onset upon EOM voltage change doubling $\mathrm{P}_{\text {tot }}$. At these resonance points, simultaneous increase of optical output power and EOM photocurrent is observed.

To further investigate properties of this resonance, constant VCSEL drive currents as indicated in Figure 7.14 were chosen to measure photocurrent and output power dependent on the applied EOM voltage (cf. Figure 7.15). For $5 \mathrm{~mA}$ VCSEL drive current, the measured photocurrent increased with an increase of the EOM reverse bias, and optical output power decreased in parallel almost monotonically, indicating mainly absorptiondriven modulation. In contrast, measurements at $6.0 \mathrm{~mA}$ and $6.2 \mathrm{~mA}$ showed a distinct onset simultaneously for photocurrent and output power, indicating fulfillment of the resonance condition by an electro-optic effect induced shift of the modulator cavity resonance. Total VCSEL section output power $\left(P_{t o t}\right)$, which is equivalent to total mirror losses of a conventional VCSEL, was calculated by adding the power that is equivalent to the photocurrent and the optical output power. The resulting $P_{t o t}$ graphs showed an almost flat behavior for the $5.0 \mathrm{~mA}$ drive current, while for higher VCSEL drive currents $P_{t o t}$ almost doubled within an ultra low EOM voltage increase of less than $100 \mathrm{mV}$. In consequence, efficient modulation of the device is only possible if a working point is chosen such that EOM voltage changes can switch the device from a resonance to an off-resonance state, and not as initially investigated in Section 7.3.1 by altering mainly the absorption characteristic. This EOM resonance modulation (ERM) cannot be explained by any 


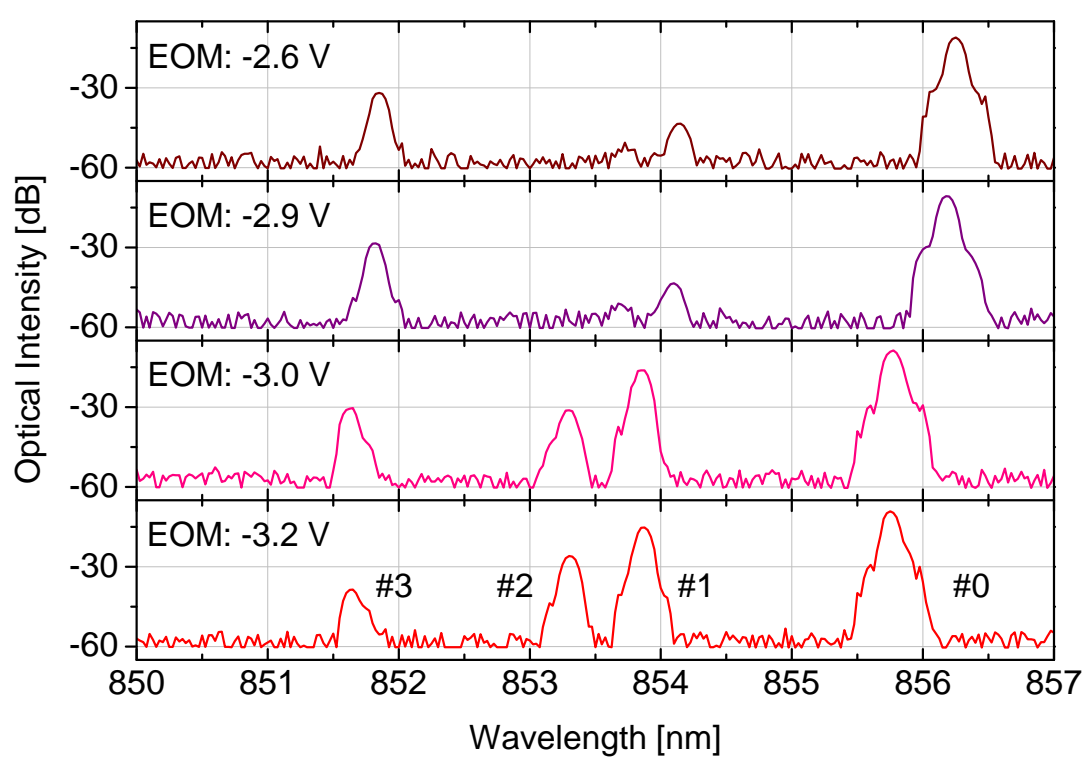

Figure 7.16: Spectrally resolved modes of EOM VCSELs at RT are shown. Drive current of VCSEL section was kept constant for all curves at $6.2 \mathrm{~mA}$ while EOM voltage was varied. An EOM voltage increase from $-2.9 \mathrm{~V}$ to $-3.0 \mathrm{~V}$ yields significant amplitude changes for modes 1 and 2 of $27 \mathrm{~dB}$ and $20 \mathrm{~dB}$, respectively, and causes all modes to slightly shift by $\leq 0.5 \mathrm{~nm}$. (Data was measured using a slightly different setup as compared to Figure 7.15 to resolve individual modes.)

absorption modulation, and is directly related to modulation of the EOM cavity by usage of the electro-optic effect.

ERM was further investigated by measuring the behavior of individual modes upon resonance onset of this multi-mode device. Figure 7.16 shows spectrally resolved optical modes of the EOM VCSEL at varied EOM voltages. For an EOM bias change from $-2.9 \mathrm{~V}$ to $-3.0 \mathrm{~V}$, a clear onset of higher order modes 1 and 2 was observed, while the fundamental mode 0 and mode 3 remained almost unchanged in intensity. All modes shifted only slightly, by less than $0.5 \mathrm{~nm}$ to shorter wavelengths. Thus total optical output increase upon ERM is driven by modes 1 and 2, which show modulation amplitudes of $27 \mathrm{~dB}$ and $20 \mathrm{~dB}$, respectively. Limiting the output to these strongly modulated modes would result in a very strong output power modulation through minimal modulation voltage change.

Small signal operation and eye diagram shown in Figure 7.9 evidenced strong parasitic limitation to about $3 \mathrm{GHz}$ of this EOM VCSEL prototype using a conventional, nonresonant working point. In contrast to conventional current modulated devices, the small signal transfer function of devices relying on photon lifetime $\left(\tau_{p}\right)$ modulation decreases with $1 / \omega$ instead of $1 / \omega^{2}$ [Avr93]. Thus the EOM VCSEL concept should enable higher modulation bandwidths. But direct small signal modulation measurements $\left(S_{21}\right)$ ensuring ERM showed a similarly limited bandwidth of $3 \mathrm{GHz}$, indicating a parasitic limitation 


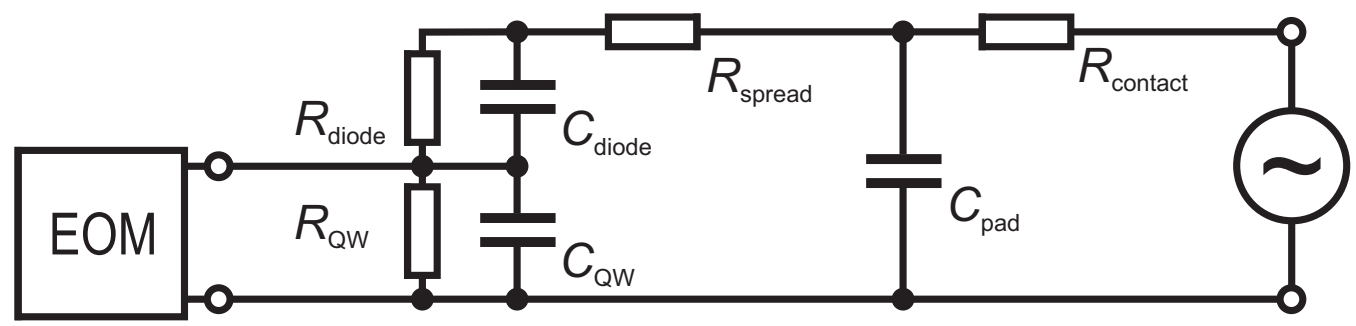

Figure 7.17: Equivalent-circuit diagram (ECD) of a characteristic device is shown. EOM section parameters are modeled like a reverse-biased PIN-diode with split elements for $\mathrm{p}$ - and n-spacecharge regions, and for the EOM-QW absorbing and refractive index changing characteristics. By fitting this ECD to the $S_{11}$ parameter data, parasitic device characteristics are assessed with high accuracy (cf. Figure 7.18).

of this prototype design. In order to assess the fundamental intrinsic bandwidth of the device and its concept, device parasitic need to be deconvoluted from data. Measurement of the device voltage reflection coefficient $\left(S_{11}\right)$ with ERM conditions allows for modeling of device characteristics by an equivalent-circuit diagram (ECD). Figure 7.17 shows the resulting ECD which enabled precise fitting of the collected $S_{11}$ data. This ECD model includes the EOM section as a reverse-biased PIN-diode. In the ECD, this diode is split into the diode capacitance of the space-charge regions of $\mathrm{p}$ - and $\mathrm{n}$-side $\left(C_{\text {diode }}\right)$ and an additional EOM-QW capacitance $\left(C_{Q W}\right)$, representing the photocurrent generating behavior. Each of these capacitors has a leakage resistance represented by $R_{\text {diode }}$ and $R_{Q W}$, respectively. Additional required lumped elements of the ECD are a spreading resistance $R_{\text {spread }}$, a contact resistance $R_{\text {contact }}$, and a pad capacitance $C_{\text {pad }}$. The results of fitting for these lumped elements of the ECD for different EOM voltages are given in Table 7.1

As expected, the outer parasitic network values $R_{\text {spread }}, R_{\text {contact }}$, and $C_{\text {pad }}$ remained nearly constant upon EOM bias variations. In contrast, the inner values showed clear trends. Space-charge capacitance $C_{\text {diode }}$ decreased consistently with wider depleted regions, while the corresponding resistance $R_{\text {diode }}$ increased significantly. As a result of the absorptionbased photocurrent generation of the EOM QWs, the corresponding leakage becomes

\begin{tabular}{l|rrrrrrr}
$\boldsymbol{U}_{E O M}$ & $\boldsymbol{C}_{Q W}$ & $\boldsymbol{R}_{Q W}$ & $\boldsymbol{C}_{\text {diode }}$ & $\boldsymbol{R}_{\text {diode }}$ & $\boldsymbol{R}_{\text {spread }}$ & $\boldsymbol{R}_{\text {contact }}$ & $\boldsymbol{C}_{\text {pad }}$ \\
\hline $\mathbf{2 . 8} \mathbf{V}$ & $55 \mathrm{fF}$ & $1040 \Omega$ & $177 \mathrm{fF}$ & $6.5 \mathrm{k} \Omega$ & $309 \Omega$ & $35.9 \Omega$ & $55.4 \mathrm{fF}$ \\
$\mathbf{3 . 0} \mathbf{V}$ & $56 \mathrm{fF}$ & $940 \Omega$ & $176 \mathrm{fF}$ & $8.1 \mathrm{k} \Omega$ & $308 \Omega$ & $35.9 \Omega$ & $55.3 \mathrm{fF}$ \\
$\mathbf{3 . 2} \mathbf{V}$ & $60 \mathrm{fF}$ & $650 \Omega$ & $173 \mathrm{fF}$ & $10.0 \mathrm{k} \Omega$ & $302 \Omega$ & $35.9 \Omega$ & $55.4 \mathrm{fF}$
\end{tabular}

Table 7.1: ECD $S_{11}$ fitting values of parasitic network as depicted in Figure 7.17 EOM VCSEL data for different EOM voltages is taken at RT with a VCSEL drive current of $6.2 \mathrm{~mA}$. 


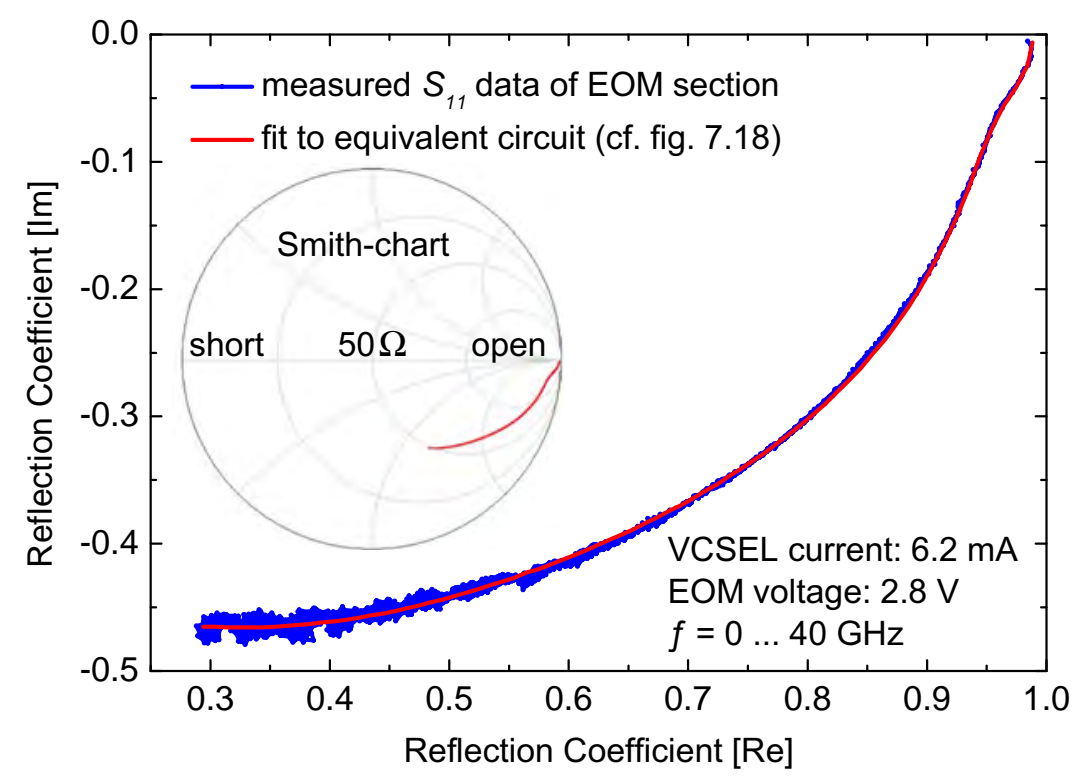

Figure 7.18: Device $S_{11}$ data and the matching fit according to ECD in 7.17 are shown. Use of this equivalent-circuit with the EOM section represented as a reverse-biased PIN-diode and split capacitances $C_{\text {diode }}$ and $C_{Q W}$ allows for an excellent full range fit from 0 to $40 \mathrm{GHz}$, including the $\mathrm{S}$-shape kink at the low frequency end. Thus, device parasitic are unveiled and can be deconvoluted to assess its intrinsic bandwidth.

inset: Smith-chart representation of measured $S_{11}$ data.

more dominant, thus resistance $R_{Q W}$ decreases. In parallel the $C_{Q W}$ increase is related to the altered refractive index and the enhanced heating by photo absorption. A fit for $2.8 \mathrm{~V}$ EOM reverse bias with this data is shown in Figure 7.18. The measured $S_{11}$ data matches perfectly over the full setup-limited frequency range up to $40 \mathrm{GHz}$, including the important S-curved kink on the low frequency side. All changes in the equivalent circuit parameters accurately reflect EOM voltage changes. Consequently, the now quantified, clearly limiting, parasitic device response can be deconvoluted from ERM $S_{21}$ small signal modulation data to uncover the intrinsic device performance. In Figure 7.19 this intrinsic $S_{21}$ data is shown. A fit employing equation 7.3 as detailed in references vE07, vE08 reveals the intrinsic potential of the device:

$$
S_{21}(\omega) \propto \frac{1}{\tau_{p 0}} \cdot \frac{\omega_{0}^{2} \tau_{p}+j \omega}{\omega_{0}^{2}-\omega^{2}+j \omega \gamma}
$$

As measured data is noise limited around $30 \mathrm{GHz}$, only data points up to this value are included in the fit. The fitted intrinsic device speed yields a photon-lifetime $\tau_{p 0}$ of 4.1 ps, a resonance-frequency $\omega_{0}$ of $2 \pi \cdot 27 \mathrm{GHz}$, and a damping-coefficient $\gamma$ of $1.5 \cdot 10^{11} \mathrm{~s}^{-1}$ at the ERM point of $6.2 \mathrm{~mA}$ and $2.8 \mathrm{~V}$. Thus, the intrinsic bandwidth of the fabricated EOM VCSELs is predicted to be $\approx 56 \mathrm{GHz}$, as shown in Figure 7.19 . This high intrinsic 


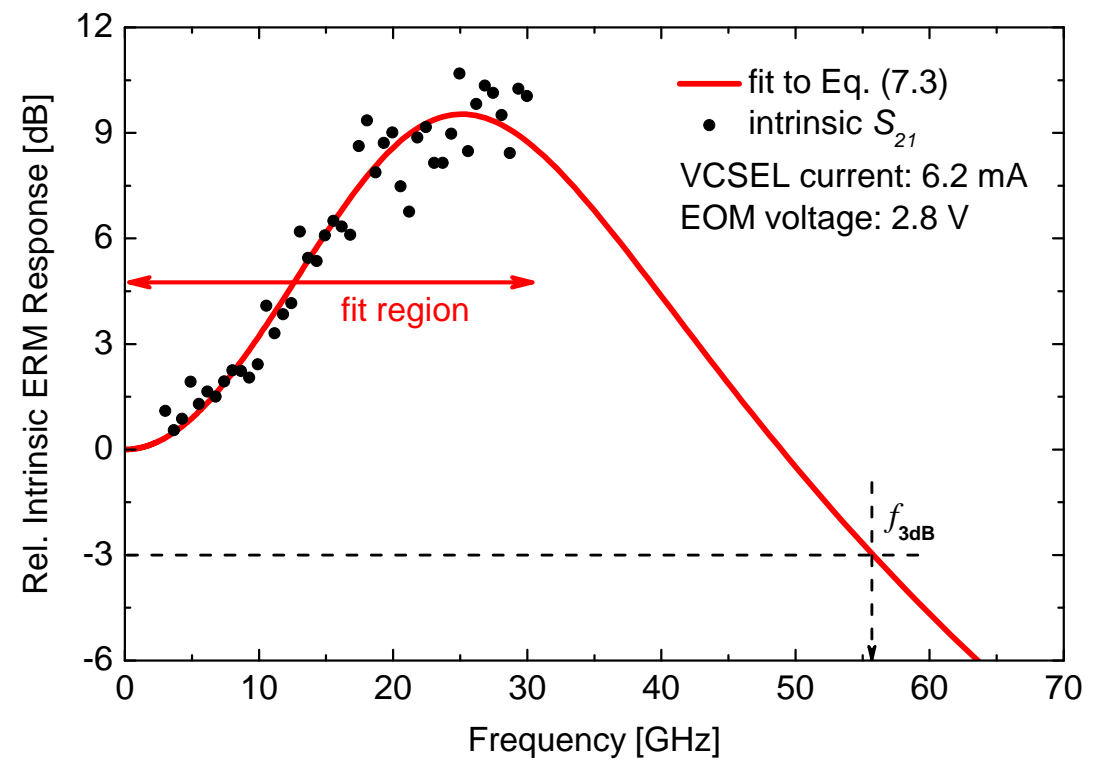

Figure 7.19: Intrinsic bandwidth of EOM VCSELs at RT is assessed by $S_{21}$ data fitting based on equation 7.3. Drive current of the VCSEL section is $6.2 \mathrm{~mA}$, EOM voltage is $2.8 \mathrm{~V}$. $S_{21}$ measurement is hitting the noise-floor at $\approx 30 \mathrm{GHz}$. Thus, the data fit region is limited to $<30 \mathrm{GHz}$. Electrical device parasitic are deconvoluted from the measured data. Bandwidth of the device is strongly limited by parasitic, but intrinsic bandwidth in ERM regime is predicted to be $\approx 56 \mathrm{GHz}$.

bandwidth evidences the potential of the monolithic EOM VCSEL concept, while the overshoot is typical for intra-cavity loss-modulated VCSELs [vE08]. Future device designs need to incorporate a well-tailored parasitic response to flatten out this characteristic overshoot without limiting device bandwidth, to achieve optimal high-speed performance.

For the present device this high intrinsic speed potential is reduced not only by absorption-induced parasitic current within the modulator, but also by high $C_{\text {diode }}$ capacitance as disclosed by the $\mathrm{ECD} 7.17$. Opposite to conventional current modulated lasers that exhibit similar confinement factors and resonance frequencies, and tend to saturate around $20-30 \mathrm{GHz}$, the EOM VCSEL concept is far from its intrinsic optimum speed. To unlock the apparent very high intrinsic performance, future device generations will require multiple design changes, such as absorption and capacitance reduction as well as high-speed processing.

The EOM VCSEL results are published in [Ger10] and the ERM analysis was recently submitted to Optics Express [Ger12]. 


\subsection{Conclusions}

Summary This work demonstrates the realization and characterization of the novel EOM VCSEL concept which monolithically integrates an electro-optic effect-based modulator within a VCSEL device. Individual optimization and simulation of all relevant parts based on separate test samples allows for accurate parameter tuning and prediction of all relevant optical EOM VCSEL characteristics. Transfer-matrix method-based simulations are used as a reliable tool for cavity resonance positioning and DBR tuning of this complex structure, which includes close to 400 layers. The precise tuning of both cavity resonances is crucial for device operation. Fundamental insight was gained with regards to EOM behavior, explaining the optical bandwidth limitation by quantifying electro-optic effect and absorption-based modulation. A resonance working point was identified allowing for much stronger EOM resonance modulation (ERM) triggered by an ultra-low modulation voltage change of less than $100 \mathrm{mV}$.

Key issues identified during this research for future EOM VCSEL devices are:

- Non-negligible absorption within the modulator section causes significant charge carrier generation.

- Parasitic currents within the EOM section need to be addressed to improve device performance.

- ERM demonstrates the strong potential for electro-optic effect-based modulation of resonant cavity coupling.

- Operating parameters for the optimal device working point are crucial for efficient modulation.

- ERM is driven by the modulation of only two out of four different modes.

- EOM VCSEL S-parameter analysis for this design shows a very high intrinsic bandwidth potential of $56 \mathrm{GHz}$.

The very demanding high-precision growth process of EOM VCSELs can be divided into optimization of individual small subsections to efficiently optimize all process parameters. Transfer-matrix method-based simulations allow for growth parameter analysis and for an accurate prediction of the spectral resonance position of a coupled cavity system.

Outlook Middle contact layer and cap layer consist of $\mathrm{GaAs}$, causing absorption for the target lasing wavelength. Future designs could employ solely lattice matched InGaP, reducing the problem of losses and device heating. This use of the non-oxidizing InGaP 
is epitaxially demonstrated for the VECSEL design in Chapter 6.3, but could necessitate some adaptation of the employed processing steps.

To assess the intrinsic high-speed potential of the EOM VCSEL concept, future designs need to address the present parasitic limitations. Absorption within the modulator can be reduced by choosing a larger spectral offset between active and modulator QWs. Significant EOM capacitance reduction is possible by a thicker EOM-cavity design combined with smaller EOM-mesa diameters and an EOM-aperture layer to reduce the effective $C_{\text {diode }}$ diameter. Such design changes could lead to an increased modulation voltage requirement that could be addressed by an increased number of EOM QWs to maintain a sufficient refractive index change.

Another important design criterion is the number of DBR pairs separating both cavities with respect to the top-most DBR thickness, and thus the confinement of the EOM cavity. These parameters are crucial for the coupling behavior of ERM operation and regulate the effective modulation and absorption strength of the EOM-QW. This needs to be taken into account to precisely tailor modulation behavior and parasitic characteristic to ensure an optimal flat broadband response. Additionally, temperature-dependent characterization could be used to fine-tune the resonance offset between both cavities post growth, and consequently to define the ERM working point.

The employed, and more robust, processing approach is not suitable for maximum modulation speed. Single mode devices are required to completely assess the potential of ERM. A reduction of EOM-mesa diameters could further reduce the modulator capacitance. Additionally, optimized processing and device mounting with minimized contact capacitances and impedance matching are required for highest modulation speeds. Future designs need to include well-tailored device parasitic to flatten out the intrinsic overshoot for midrange frequencies, and to ensure a flat broadband response.

Beyond these direct improvements, future optimization of the EOM section requires further investigations on the modulating behavior of the MQW system, novel design approaches, or advanced modulation concepts. Particularly coupled compared to uncoupled QW systems could be advantageous. Additionally, type II hetero junctions separating holes and electrons could prove to be most efficient. A completely different design approach already mentioned in [Shc08] eliminates the need for a second cavity by positioning the VCSEL emission wavelength at the edge of the DBR stop band. However, this DBR stop band modulation could be technically demanding and would require extensive epitaxial process and design optimization. Another important concept is the simultaneous modulation of $j$ and $\tau_{p h}$, as theoretically predicted by Avrutin et al. [Avr93]. This could be realized by a coupled frequency-dependent modulation of VCSEL-section current and EOM voltage. 
The EOM VCSEL concept offers a new mechanism for generating high-speed devices by eliminating the requirement for direct modulation. By using established growth and processing technologies, this novel concept is compatible with large-scale manufacturing processes. MOVPE growth demonstrated the feasibility of this demanding design, while proving the excellent epitaxial growth stability for hundreds of layers. The presented ultra-low voltage EOM modulation enables fabrication of cost-efficient systems using established CMOS driver circuits. Future EOM VCSELs can potentially outperform any conventional direct modulated devices, which are inherently limited by the required drive current density. 



\section{Chapter 8}

\section{Summary and Outlook}

Summary MOVPE of long wavelength InGaAs/GaAs QDs emitting around $1.3 \mu \mathrm{m}$ was systematically studied. Growth processes for three completely different laser concepts based on QWs, QDs, and SMLs were developed and devices were realized. In the course of this work about 2500 different MOVPE samples were grown, measured, and characterized. Among these were:

- Multiple QD lasers with $A l G a A s$ and InGaP designs that exhibited record low thresholds and emission wavelengths up to $1.3 \mu \mathrm{m}$.

- First MOVPE grown SML and QD-based VECSELs, emitting at $950 \mathrm{~nm}, 1040 \mathrm{~nm}$, and $1210 \mathrm{~nm}$ were fabricated. High-power, high-brilliance output and excellent temperature stability were demonstrated.

- Realization of the novel EOM VCSEL concept based on a QW double-cavity design unveiled an ultra-high intrinsic bandwidth potential surpassing direct modulated VCSEL.

For the systematic study of QD growth and QD stacking processes for long wavelength applications, a standardized PL test structure design with constant excitation volume was adopted. Multiple computer-based tools were implemented to deal with the substantial number of samples.

Based on an existing InGaAs/GaAs QD growth process, the influence of individual growth parameters on the QD luminescence characteristic was studied in detail, and the QD growth process was developed further. A series of PL test samples revealed the important role of the V/III ratio during overgrowth of QDs. A strong influence of the V/III ratio on QD properties was determined. Reduced V/III ratios improved the integration of QDs into the GaAs matrix crystal, enabling a reduced FWHM, longer emission wavelength, and a significantly improved QD stacking ability. The improved QD properties are attributed to a reduction of point defects in the proximity of the QD layer. In consequence, the vacancy-driven indium segregation was suppressed. Effects of the V/III ratio on QD properties were found not only for QD deposition and the capping process itself, but also for all layers deposited subsequent to a QD layer. This finding is explained by a group three vacancy rich growth surface for high arsenic partial pressures, leading to enhanced indium 
segregation out of the QDs. Through these improvements of the QD growth process, the successful complete suppression of QD emission blue shift upon overgrowth and annealing was achieved, even for long wavelength QDs at $1.3 \mu \mathrm{m}$ [Str08a]

Edge-emitting lasers based on this advanced QD growth process were fabricated, achieving ultra-low threshold values of only $66 \mathrm{~A} / \mathrm{cm}^{2}$ at $1.25 \mu \mathrm{m}$ [Str06, Ger07]. Additional to the conventional AlGaAs-based design, a completely aluminum-free design based on lattice matched In $\mathrm{GaP}$ cladding was developed and realized, achieving an extremely low transparency-current density of only $35 \mathrm{~A} / \mathrm{cm}^{2}$ at the same wavelength.

Complete suppression of any unintended blue shift during QD stacking and cladding growth enabled the realization of one of the first MOVPE grown 1.3 $\mu \mathrm{m}$ QD lasers. In contrast to other reports, the presented growth process was carried out without the need for additional surfactant materials such as antimony, and was solely based on a systematically improved QD growth process.

Using MOVPE the first SML-based and the first QD-based optically pumped VECSELs were designed and fabricated. SML GS emission was used for the generation of VECSELs emitting at $0.95 \mu \mathrm{m}$ and $1.04 \mu \mathrm{m}$, with $\mathrm{CW}$ output powers reaching up to $1.4 \mathrm{~W}$ [Ger08b]. QD ES transitions were employed to realize a QD VECSEL design at the same $1.04 \mu \mathrm{m}$ wavelength [Str08b]. A low-loss design was developed to enable GS QD lasing at $1.21 \mu \mathrm{m}$, achieving an ultra-low threshold value of only $2.4 \mathrm{~kW} / \mathrm{cm}^{2}$. Use of the very broad QD gain spectrum enabled an extremely temperature stable VECSEL performance, clearly evidencing the advantage of QD gain media for this laser type [Ger08a, Ger08c].

The novel concept of a VCSEL with a monolithically integrated electro-optic modulator was realized as a twin cavity QW design. Close to 400 total layers resulted in a device thickness of $12 \mu \mathrm{m}$, while the resonance wavelength offset between both cavities has been kept to less than $1 \mathrm{~nm}$. This very demanding epitaxial design was fabricated by a single MOVPE growth run, and shows lasing up to $\approx 8 \mathrm{~mW}$ output power [Ger10]. An in-depth analysis disclosed a resonance working point enabling $27 \mathrm{~dB}$ modulation of an individual mode by an ultra-low voltage change of less than $100 \mathrm{mV}$. While modulation bandwidth of this prototype design was limited by parasitic to $3 \mathrm{GHz}$, a very high intrinsic bandwidth potential of $56 \mathrm{GHz}$ could be revealed by ensuring EOM resonance modulation. This finding evidences the huge potential of the EOM VCSEL concept for future ultra-highspeed devices.

Outlook Nowadays the majority of semiconductor photonic devices rely on QWs as active nanostructures. Since all applications have a unique set of requirements, individual epitaxial designs are required. Thus QW active media are often not the optimum choice for all kinds of devices. Future highly optimized semiconductor laser concepts require not 
only advanced structural designs, but also tailor-made active media solutions to achieve maximum performance.

As presented in this work QDs, which are already entering the mass market, offer clear advantages. These include thermal stability for distinct device designs such as VECSEL. Similar advantages as those for QD VECSEL can be anticipated for all other laser concepts which through their design have an already fixed and predefined emission wavelength, and will consequently benefit from the spectrally broad QD emission e.g. DFB lasers. Compared to QW-based VECSELs, research on the QD-based variant is rather new. Emerging reports can already show further improvements on QD-based VECSEL devices [But09, Rau10, Alb11]. Future advancements need to target two areas: Firstly, improvement of the active QD gain media by closer stacking of dozens of layers with highest QD densities, including possible strain compensation; and, secondly, advancing the VECSEL design itself, e.g. by improved pump power balancing through use of a double stop band DBR, or improving heat removal by advanced processing such as substrate removal.

Besides QWs and QDs, a better understanding of SML growth and physics could close the gap between QW and QD characteristics to enable tailor-made optimum optical properties for all photonic device applications. Advanced SML growth processes would need to reduce lateral coupling by controlling island sizes and distribution, while maintaining vertical correlation. Lateral charge carrier confinement can be supported by choosing matrix materials with larger band gap energies than $G a A s$, such as $A l G a A s$ or In $G a P$. Thus, investigations for future SML MOVPE growth processes should not be restricted to the InAs/GaAs approach, but rather be open-minded towards other material combinations. This includes surface mobility mediating substances such as antimony, which could improve control of the InAs-island formation.

Novel innovative concepts such as EOM VCSELs can overcome physical limits of conventional direct modulated VCSELs, thereby pushing modulation speed to new limits. This research revealed multiple possibilities for developing the EOM VCSEL concept further. Limitations of the present design, such as absorbing layers and strong parasitic effects need to be eliminated. For this purpose, absorbing $\mathrm{GaAs}$ can be replaced by lattice matched InGaP, and the EOM capacitance can be significantly reduced by introducing an aperture layer, increasing the cavity length, and reducing the EOM mesa diameter. Spectral offset of EOM QWs should be increased to reduce losses, while enabling an increased number of modulator QWs. High-speed compatible processing is indispensable including impedance matching, minimized contact capacitances, and single mode operation. Advanced EOM VCSEL designs could include type II or coupled QW systems to boost the attainable EOM effect. Sophisticated driving concepts could target the simultaneous modulation of $j$ and $\tau_{p h}$ for ultimate performance [Avr93]. 



\section{Appendix A}

\section{Additional Methods}

\section{A.1 MOVPE growth}

\section{A.1.1 In-situ control}

Process control is performed by the commercially available optical in-situ sensor Laytec EpiRAS which is upgraded for most recent samples by the TT-option (an additional pyrometric sensor). This sensor system is based on a white light source (xenon arc lamp) and a free-air light path to the wafer, and back to a monochromator/detector unit (280 - $800 \mathrm{~nm}$ ). Light is reflected twice at the wafer surface; on its way to a concave anti-wobbling mirror with focal point on the wafer surface, and on its way back to the monochromator. Thus the variable light path directly compensates for any tilted reflection or defocusing caused by wobbling of the rotating wafer mount.

\section{Reflectance anisotropy spectroscopy}

Reflectance anisotropy spectroscopy (RAS) uses linearly polarized light to measure anisotropy between the main crystal axes. As the substrate rotates within the reactor all possible angles between polarization direction and crystal axes are measured. Due to the cubic crystal geometry of GaAs bulk material it is isotropic for RAS, whereas the anisotropic signal originates solely from the non-isotropic surface reconstruction. The employed RAS system is based on an photoelastic-modulator configuration as detailed in [Asp88]. For most samples, time resolution is crucial, thus detection wavelength is set to $2.65 \mathrm{eV}$ instead of measuring full spectra through the whole monochromator range. The chosen energy is related to the arsenic dimer which is part of the GaAs surface reconstruction and is especially sensitive to the deposition of $I n A s$ monolayers required for QD growth [Ste97].

\section{Reflectometry}

Reflectometry uses the same signal as RAS. Due to the absorption characteristics of the $A l_{x} G a_{x-1} A s$-system the signal can originate from several layers close to the surface at once. Thus Fabry-Pérot oscillations are observed at hetero interfaces which can be used to calculate the current epitaxial growth rate. In addition to surface sensitive RAS 
measurements, reflectometry is used for all growth runs to monitor growth progress, to analyze process errors, and to check in-situ surface roughness.

\section{Pyrometry}

In order to gain direct access to the wafer surface temperature the EpiRAS system was recently upgraded with a pyrometry system. This system uses the same light path but employs an additional LED light source for a reflectivity measurement at $950 \mathrm{~nm}$. Data is corrected for emissivity by calculating the difference between true wafer emission and emission from a perfect black body. Thus, correct wafer temperature measurements are possible independently of possible hetero interface multi reflections and actual growth temperature. When properly calibrated, this system allows for a more precise wafer surface temperature control without the intrinsic temperature offset of the MOVPE system. Calibration is carried out by monitoring the silicon-aluminum eutectic transition point at $577^{\circ} \mathrm{C}$. At QD growth temperatures around $500^{\circ} \mathrm{C}$ the signal is weak, thus accuracy is reduced to $\approx 5^{\circ} \mathrm{C}$, while at higher growth temperatures $\left(600^{\circ} \mathrm{C}\right.$ and above) an accuracy of $\approx 1^{\circ} \mathrm{C}$ is achieved. By adding the pyrometric sensor to the MOVPE system, runto-run temperature control is achieved, eliminating the need for individual temperature calibrations in most cases.

\section{A.1.2 Process design tools}

Besides the employed commercial Aixtron CACE operating software for the MOVPE system, multiple tools were developed to facilitate epitaxy-process programming and to minimize sources of error. These tools were calibrated to allow for an accurate prediction of final layer structures and layer thickness. Such layer assembly calculations are based solely on parameters of the employed precursors, such as source flows, pressures and temperatures, as well as the reactor temperature, total flow value, and deposition time.

\section{Schätzer}

The Schätzer is an Excel-based tool enabling calculations of growth rate and layer thickness, as well as ternary material composition and V/III ratio. Results are based on growth parameters, source material constants, and calibrated growth efficiencies of the employed precursors. Schätzer significantly simplifies programming of complex epitaxial layer assemblies and minimizes growth rate and compositional calculation errors.

\section{Previewer}

The Previewer-tool was initially scripted by André Strittmatter as a standalone application. This tool is based on a parser for Aixtron epitaxy-process files and uses the same formulas 
as the Schätzer tool to directly calculate layer parameters and to plot all informations on the whole layer structure, including growth relevant parameters such as total flow or V/III ratio. Additionally, Previewer enables one to go step-by-step through the epitaxy-process file for debugging and analysis. Previewer was recently integrated into the Online lab-book by David Quandt, Jan-Hindrik Schulze, and André Strittmatter. This allows for the direct integrated display of layer structures within the online database.

\section{Online lab-book}

Based on the need for a comprehensive compilation of all growth-relevant data and all characterization results, combined with the possibility of a universal search engine, the idea of an online lab book was developed. The basic idea, requirements, and the initial implementation were developed for several years by the author and coded by Jürgen Brandt as part of a student research work. Programming of the actual completely redesigned version was mainly conducted by Jan-Hindrik Schulze and David Quandt. The online lab-book is entirely web browser-based, employing a MySQL database and $P H P$ written dynamic content. As previously mentioned, the Previewer is now directly integrated. Image files for data such as atomic force microscope (AFM) or Nomarski micrographs can be uploaded. An import filter for ASCII data from PL and in-situ measurements exists, and data curves can be directly plotted, magnified and compared within the web browser using JavaScript. LabVIEW-based measurement setups such as Hall characterization or PL can directly upload results into the database. All maintenance entries or growth runs are tagged according to their needs to allow for a comprehensive search within the whole database. Sample search attributes include indicators such as project, growth series, or wafer batch number, to easily identify interrelationships. Due to its universal concept the online labbook is easily extendable for additional data sources and new project requirements. As a work-in-progress, novel features are continuously being added while two MOVPE systems steadily produce new input data.

\section{A.2 Sample characterization}

\section{A.2.1 Microscopic characterization}

Surface characterization often indicates growth- or wafer-related problems. As the dimensions of crystalline defects can increase to a macroscopic scale during growth they become visible during optical microscopy. This effect enables differential interference contrast microscopy (Nomarski microscope), which is very sensitive to surface roughness, to uncover epitaxial growth or wafer problems. Thus the employed Carl Zeiss universal polarization-microscope was used to quickly check post-growth epitaxial layer quality. 
To assess sample properties on a lateral nanometer scale, a variety of other non-optical microscope techniques are available.

\section{Atomic force microscopy}

Atomic force microscope (AFM) measurements allow for height resolutions up to individual atomic layers, whereas lateral resolution is limited by the tip radius of $\approx 20 \mathrm{~nm}$. Thus QD shape representation is always a convolution of QD geometry, employed SiN tip-shape, and the physical interaction mechanisms between both. QD geometry changes during capping and GRI, which is inevitable while cooling down from growth temperature. Consequently, AFM measurements are not used to investigate changes in QD shape, but are instead employed to precisely characterize surface quality and to quantify QD densities.

\section{Scanning electron microscopy}

A commercially available Zeiss Ultra 55 scanning electron microscope (SEM) was used to investigate surface defects and complex structures in a cross-sectional view. The maximum attainable resolutions of the employed system are $1.0 \mathrm{~nm}$ at $15 \mathrm{kV}$ or $1.7 \mathrm{~nm}$ at $1 \mathrm{kV}$. SEM micrographs assisted in tracing back surface defects to the epilayer-wafer interface. In this manner, imperfect wafer charges with defects or contamination not visible prior to growth were identified. Additionally, growth homogeneity was investigated for DBR structures and final devices were crosschecked for vertical structure and growth quality.

\section{A.2.2 Volume-crystal characterization}

Growth parameter calibrations and impurity tracing require the measurement of multiple layers and thick structures (>100 nm). Crystal quality is further investigated and quantified by intrinsic charge carrier concentration and mobility measurements.

\section{X-ray diffraction}

For XRD measurements, a commercially available Philips X'pert Pro MRD setup was employed. An hybrid 4-bounce germanium-monochromator reduced radiation of the X-ray

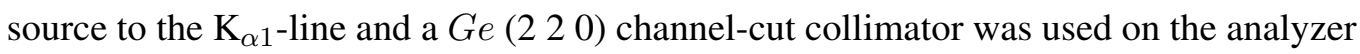
side. All XRD test samples were measured with omega-2-theta scans of the $G a A s$ ( $\left.\begin{array}{lll}0 & 0 & 4\end{array}\right)$ reflex and subsequently fitted with the commercial PANalytical X'pert epitaxy software. The fitting routine was based on a solver for Takagi-Taupin equations of the dynamic scattering theory [Tak62, Tau64, Tak69].

Measurements of ternary layers are used to tune compositions, which is especially important to achieve lattice matching of In GaP on $G a A s$. SL samples as described in A.3 are grown and measured to calibrate epitaxial growth rates. As stacked active QD layers 
also represent a SL structure, the QD period in such structures can be determined with high accuracy. More detailed insight into XRD methodology and XRD QD-measurements is given by Krost et al. [Kro96a, Kro96b].

\section{Charge carrier measurements}

Doping levels and electron mobilities are measured using the Hall effect employing the $L$. $J$. van der Pauw-method [vdP59]. Samples as described in A.3 are prepared for doping level characterization and standard material characterization of electronic properties. As this measurement relies on resistivity measurements of the manually soldered ohmic contacts, metal contact size and quality influence the measurement error $(\approx 20 \%)$. Additionally, the measurement range is limited by the resolution of the voltmeter and the range of the current source to a sample resistivity of $<10^{7} \Omega$. Thus, very low doping levels can be out of the range of this measurement method. The setup includes a magnet with a magnetic field strength of $0.42 \mathrm{~T}$ and the possibility for measurements at $77 \mathrm{~K}$ through the use of liquid nitrogen. CV-measurements are used to cross-check results of selected samples or for samples on doped substrates. Characteristics of doped multi-layer samples are determined using a commercial Bio-Rad PN430OPC etch-CV profiler.

\section{Secondary ion mass spectroscopy}

Vertical resolution of secondary ion mass spectroscopy (SIMS) is systematically limited to $\approx 5 \mathrm{~nm}$ which is insufficient for investigations of nanostructures such as QWs and QDs. In contrast, the impurity detection limit of $\approx 10^{5}$ up to $10 \mathrm{ppb}$ is excellent. Thus SIMS is used to investigate unintended dopant diffusion in complex device structures. The employed system is a commercial Atomika SIMS-6500 system equipped with oxygen, argon, and cesium ion-sources. Depending on the investigated elements, either $\mathrm{O}_{2}^{+}$or $\mathrm{Cs}^{+}$ions with accelerations energies between $6 \mathrm{keV}$ and $13 \mathrm{keV}$ are used. Further information on the physics of SIMS can be found in the following textbooks [Mc'75, Brü80, Che87b].

\section{A.3 Sample structure designs}

\section{XRD test structures}

$\mathrm{X}$-ray diffraction (XRD) measurements are used for compositional and growth rate calibrations. Ternary compositions are calibrated by test samples with bulk semiconductor layers while growth rate information is determined from superlattice (SL) structures. Such SL test samples consist usually, but not necessarily, of two alternating epitaxial layers e.g. stacked QD layers can be measured to directly determine the period of active layers. As already described in 3.1.1, a QD growth process such as this consists of numerous growth steps at 
varying temperatures. Calibration of the growth rates for all layers separately is costly and time-consuming, while direct measurement of the whole QD stack results in more accurate active layer period values by including all intermediary growth steps at once.

\section{Hall test structures}

Test samples for Hall measurements using the L. J. van der Pauw-method [vdP59] are bulk layers of one to three microns with constant doping levels. Aluminum-containing samples are capped by an undoped $G a A s$ layer $\approx 15 \mathrm{~nm}$ thick to prevent oxidation of the surface. Prior to measurement, $\approx 1 \mathrm{~cm}^{2}$ pieces are cleaved and small indium contacts are soldered manually near the corners. Samples are subsequently annealed for about $15 \mathrm{~min}$ at $\approx 400^{\circ} \mathrm{C}$ on a hot plate to generate ohmic contacts.

\section{Other test structures}

For DBR and cavity resonance calibrations, test samples with reduced period numbers are grown to measure surface reflectivity. Subsequent transfer matrix simulations are used to tune growth parameters.

For AFM investigations, samples are cleaved to $\approx 1 \mathrm{~cm}^{2}$ pieces and bonded with conductive silver to equivalently large metal plates. This enables mounting on the magnetic sample holder of the AFM. For QD density investigations, special AFM test samples are grown. The QD layer within these samples is covered by only a few nanometers of $\mathrm{GaAs}$ to conserve QDs during cooling of the reactor.

\section{A.4 Transfer matrix simulation}

Commercial Wolfram Mathematica software was used to compute simulations of complex layer designs employing the transfer matrix method [Bor00]. Algorithms initially implemented by André Strittmatter were used to calculate reflection spectra and optical standing wave pattern from an input layer stack. The resulting calculations were used to tune DBR and cavity properties or to calculate 1-dimensional optical field distributions in waveguides. The fitting of simulation model values to optical reflection data was used as a fast and accurate characterization tool for test samples and complete device structures.

\section{A.5 Processing and device characterization}

All electrically pumped laser devices were processed and characterized on-site. Initial characterization was done directly post-growth by PL and/or reflectivity measurements. Processing and characterization of edge emitters was carried out by Kristijan Posilovic and 
Thorsten Kettler. Electro-optically modulated vertical emitters were processed by Alex Mutig using standard lithography techniques as detailed in [Hop07]. Device performance measurements were performed by Alexey Nadtochiy and Sergey Blokhin.

\section{A.5.1 Edge-emitter fast-process}

The edge-emitter Schnellprozess (fast-process) was employed to produce ultra-fast results for the first feedback with regards to material and process quality. Using this Schnellprozess, the first laser parameters can already be made available on the $2^{\text {nd }}$ day post-growth. This is achieved through a minimized number of processing steps to fabricate broad-area laserdiodes. Results from such devices allow for fast feedback on growth quality and device design. Processing steps of this Schnellprozess are outlined in the following:

- Using standard photolithographic techniques ohmic Ti/Pt/Au p-contacts are established on the wafer surface by electron-beam evaporation (width $100 \mu \mathrm{m}$ ).

- Broad-area mesa-stripes are wet-chemically etched using the already defined metallic p-contacts as a masking layer.

- Wafer lapping is carried out to thin the structure to a total thickness of about $100 \mu \mathrm{m}$ to $150 \mu \mathrm{m}$.

- Metallization of the entire underside of the wafer is done with $\mathrm{Ni} / \mathrm{Au}_{0.88} \mathrm{Ge}_{0.12} / \mathrm{Au}$ using a thermal evaporator to establish an ohmic n-contact.

- Groups of lasers with lengths ranging from $0.3 \mathrm{~mm}$ up to $8 \mathrm{~mm}$ are cleaved. To speed up the process, laser bars of 5 or 10 edge-emitters are directly measured without individually separating devices or sophisticated mounting techniques.

A more detailed description of the Schnellprozess can be found in [Rib02, Ket10]. All edge-emitter results depicted within this work are fabricated employing this Schnellprozess.

\section{A.5.2 Edge emitter characterization}

For device characterization, cleaved laser bars are fixed on a gold-plated copper block used as an n-contact. Individual lasers are addressed using a golden needle as a p-contact. A pulsed current source with $2 \mathrm{~A}$ at $80 \mathrm{Ohm}$ is used. Pulse widths range from $300 \mathrm{~ns}$ to $800 \mathrm{~ns}$ at repetition rates of $1 \mathrm{kHz}$. Detection is carried out by an Ulbricht sphere including independent photo diodes with separate spectral filters. The two independent photo currents enable simultaneous monitoring of lasing power and wavelength. As an alternative, a fiber-coupled optical spectrum analyzer is used. 



\section{References}

[Ada85] S. Adachi, GaAs, AlAs, and $A l_{x} G a_{1-x} A s$ Material parameters for use in research and device applications, Journal of Applied Physics 58 (3), R1 (1985), ISSN 00218979.

[Alb11] A. R. Albrecht, C. P. Hains, T. J. Rotter, A. Stintz, K. J. Malloy, G. Balakrishnan, and J. V. Moloney, High power $1.25 \mu \mathrm{m}$ InAs quantum dot vertical external-cavity surface-emitting laser, Journal of Vacuum Science \& Technology B: Microelectronics and Nanometer Structures 29 (3), 03C113 (2011). ISSN 10711023.

[Alf69] Z. I. Alferov, V. M. Andreev, V. I. Korolkov, E. L. Portnoi, and D. N. Tretyako, Coherent radiation of epitaxial heterojunction structures in AlAs-GaAs system, Soviet Phys.-Semiconductors USSR 2 (10), 1289 (1969), ISSN 0038-5700.

[Alf71] Z. I. Alferov, V. M. Andreev, D. Z. Garbuzov, Y. V. Zhilyaev, E. P. Morozov, E. L. Portnoi, and V. G. Trofim, Investigation of the influence of the AlAs-GaAs heterostructure parameters on the laser threshold current and realization of continuous emsission at room temperature, Soviet Phys.-Semiconductors USSR 4 (9), 1573 (1971), ISSN 0038-5700.

[Ara82] Y. Arakawa, Multidimensional quantum well laser and temperature dependence of its threshold current, Applied Physics Letters 40 (11), 939 (1982), ISSN 00036951.

[Asa86] M. Asada, Y. Miyamoto, and Y. Suematsu, Gain and the threshold of threedimensional quantum-box lasers, IEEE Journal of Quantum Electronics 22 (9), 1915 (1986), ISSN 0018-9197.

[Asc05] A. Aschwanden, D. Lorenser, H. J. Unold, R. Paschotta, E. Gini, and U. Keller, $10 \mathrm{GHz}$ passively mode-locked external-cavity semiconductor laser with $1.4 \mathrm{~W}$ average output power, Applied Physics Letters 86 (13), 131102 (2005).

[Asp86] D. E. Aspnes, S. M. Kelso, R. a. Logan, and R. Bhat, Optical properties of $A l_{x} G a_{1-x} A s$, Journal of Applied Physics 60 (2), 754 (1986), ISSN 00218979.

[Asp88] D. E. Aspnes, Application of reflectance difference spectroscopy to molecularbeam epitaxy growth of GaAs and AlAs, Journal of Vacuum Science \& Technology A: Vacuum, Surfaces, and Films 6 (3), 1327 (1988), ISSN 07342101. 
[Asr01] L. V. Asryan, M. Grundmann, N. N. Ledentsov, O. Stier, R. a. Suris, and D. Bimberg, Maximum modal gain of a self-assembled InAs/GaAs quantum-dot laser, Journal of Applied Physics 90 (3), 1666 (2001), ISSN 00218979.

[Avr93] E. A. Avrutin, V. B. Gorfinkel, S. Luryi, and K. A. Shore, Control of surfaceemitting laser diodes by modulating the distributed Bragg mirror reflectivity: Small-signal analysis, Applied Physics Letters 63 (18), 2460 (1993), ISSN 00036951.

[Bab01] A. Babiński, J. Jasiński, R. Bozek, A. Szepielow, and J. M. Baranowski, Rapid thermal annealing of InAs/GaAs quantum dots under a GaAs proximity cap, Applied Physics Letters 79 (16), 2576 (2001)

[Bac07] F. Bachmann, P. Loosen, and P. R., High-Power Diode Lasers Technology and Applications, Springer Science and Business Media, New York (2007)

[Ban10] K.-Y. Ban, S. P. Bremner, G. Liu, S. N. Dahal, P. C. Dippo, A. G. Norman, and C. B. Honsberg, Use of a GaAsSb buffer layer for the formation of small, uniform, and dense InAs quantum dots, Applied Physics Letters 96 (18), 183101 (2010)

[Bey05] S.-S. Beyertt, M. Zorn, T. Kübler, H. Wenzel, M. Weyers, A. Giesen, G. Tränkle, and U. Brauch, Optical in-well pumping of a semiconductor disk laser with high optical efficiency, IEEE Journal of Quantum Electronics 41 (12), 1439 (2005), ISSN 0018-9197.

[Bey07] S.-S. Beyertt, U. Brauch, F. Demaria, N. Dhidah, A. Giesen, T. Kubler, S. Lorch, F. Rinaldi, and P. Unger, Efficient Gallium Arsenide Disk Laser, IEEE Journal of Quantum Electronics 43 (10), 869 (2007), ISSN 0018-9197.

[Bim92] D. Bimberg, F. Heinrichsdorff, R. K. Bauer, D. Gerthsen, D. Stenkamp, D. E. Mars, and J. N. Miller, Binary AlAs/GaAs versus ternary GaAlAs/GaAs interfaces: A dramatic difference of perfection, Journal of Vacuum Science \& Technology B 10 (4), 1793 (1992)

[Bim98] D. Bimberg, M. Grundmann, and N. N. Ledentsov, Quantum Dot Heterostructures, John Wiley \& Sons, Chichester (1998).

[Bim08] D. Bimberg (editor), Semiconductor Nanostructures, Springer, Berlin Heidelberg (2008).

[Blo09] S. Blokhin, J. Lott, A. Mutig, G. Fiol, N. Ledentsov, M. Maximov, A. Nadtochiy, V. Shchukin, and D. Bimberg, Oxide-confined $850 \mathrm{~nm}$ VCSELs operating at bit rates up to 40 Gbit/s, Electronics Letters 45 (10), 501 (2009), ISSN 0013-5194. 
[Bor00] M. Born, E. Wolf, A. Bhatia, P. Clemmow, D. Gabor, A. Stokes, A. Taylor, P. Wayman, and W. Wilcock, Principles of Optics: Electromagnetic Theory of Propagation, Interference and Diffraction of Light, Cambridge University Press, 7 edition (2000), ISBN 9780521784498

[Brü80] O. Brümmer, Handbuch Festkörperanalyse mit Elektronen, Ionen und Röntgenstrahlen, Vieweg (1980), ISBN 9783528083984

[But09] M. Butkus, K. G. Wilcox, J. Rautiainen, O. G. Okhotnikov, S. S. Mikhrin, I. L. Krestnikov, A. R. Kovsh, M. Hoffmann, T. Südmeyer, U. Keller, and E. U. Rafailov, High-power quantum-dot-based semiconductor disk laser, Optics Letters 34 (11), 1672 (2009), ISSN 0146-9592.

[Ca109] S. Calvez, J. Hastie, M. Guina, O. Okhotnikov, and M. Dawson, Semiconductor disk lasers for the generation of visible and ultraviolet radiation, Laser \& Photonics Reviews 3 (5), 407 (2009), ISSN 1863-8899.

[Cam06] G. K. Cambron, The Multimedia Transformation, Optical Fiber Communication Conference/National Fiber Optic Engineers Conference (2006)

[Cas73] H. C. Casey, Variation of minority-carrier diffusion length with carrier concentration in GaAs liquid-phase epitaxial layers, Journal of Applied Physics 44 (3), 1281 (1973), ISSN 00218979.

[Cha64a] L. Chang and H. Caseyjr, Diffusion and solubility of zinc in indium phosphide, Solid-State Electronics 7 (6), 481 (1964), ISSN 00381101.

[Cha64b] L. L. Chang and G. L. Pearson, Diffusion and Solubility of Zinc in Gallium Phosphide Single Crystals, Journal of Applied Physics 35 (2), 374 (1964), ISSN 00218979.

[Cha64c] L. L. Chang and G. L. Pearson, Diffusion Mechanism of Zn in GaAs and GaP Based on Isoconcentration Diffusion Experiments, Journal of Applied Physics 35 (6), 1960 (1964), ISSN 00218979.

[Cha04] F. Chang, J. Lee, and H. Lin, Low threshold current density $1.3 \mu \mathrm{m}$ InAs/InGaAs quantum dot lasers with InGaP cladding layers grown by gas-source molecularbeam epitaxy, Electronics Letters 40 (3), 179 (2004), ISSN 00135194.

[Che87a] C. H. Chen, C. A. Larsen, and G. B. Stringfellow, Use of tertiarybutylarsine for GaAs growth, Applied Physics Letters 50 (4), 218 (1987).

[Che87b] V. Cherepin, Secondary ion mass spectroscopy of solid surfaces, VNU Science Press (1987), ISBN 9789067640787. 
[Che95] Y. M. Cheng, R. W. Herrick, P. M. Petroff, M. K. Hibbs-Brenner, and R. A. Morgan, Degradation studies of proton-implanted vertical cavity surface emitting lasers, Applied Physics Letters 67 (12), 1648 (1995)

[Che96] Y. Cheng, R. Herrick, P. Petroff, M. Hibbs-Brenner, and R. Morgan, Degradation mechanisms of vertical cavity surface emitting lasers, IEEE International Reliability Physics Symposium, 1996. 34th Annual Proceedings p. 211 -213 (1996).

[Che10] A. Chernikov, J. Herrmann, M. Scheller, M. Koch, B. Kunert, W. Stolz, S. Chatterjee, S. W. Koch, T. L. Wang, Y. Kaneda, J. M. Yarborough, J. Hader, and J. V. Moloney, Influence of the spatial pump distribution on the performance of high power vertical-external-cavity surface-emitting lasers, Applied Physics Letters 97 (19), 191110 (2010).

[Cho97] K. Choquette, K. Geib, C. Ashby, R. Twesten, O. Blum, H. Hou, D. Follstaedt, B. Hammons, D. Mathes, and R. Hull, Advances in selective wet oxidation of AlGaAs alloys, IEEE Journal of Selected Topics in Quantum Electronics 3 (3), 916 (1997), ISSN 1077-260X.

[Cho98] S. J. Choi, J. H. Seo, J. H. Lee, and K. S. Seo, Low Doped P-Type AlGaAs Buffer Layers Grown by Metalorganic Chemical Vapor Deposition Using Intrinsic Carbon Doping Method, Japanese Journal of Applied Physics 37 (Part 2, No. 4A), L363 (1998)

[Chu09] S. L. Chuang, Physics of optoelectronic devices, John Wiley \& Sons, second edition (2009), ISBN 978-0-470-29319-5.

[Co195] L. Coldren and S. Corzine, Diode Lasers and Photonic Integrated Circuits, John Wiley \& Sons, New York (1995).

[Cos06] G. Costantini, A. Rastelli, C. Manzano, P. Acosta-Diaz, R. Songmuang, G. Katsaros, O. Schmidt, and K. Kern, Interplay between Thermodynamics and Kinetics in the Capping of InAs/GaAs(001) Quantum Dots, Physical Review Letters 96 (22), 226106 (2006), ISSN 0031-9007.

[Dep88] D. G. Deppe and J. N. Holonyak, Atom diffusion and impurity-induced layer disordering in quantum well III-V semiconductor heterostructures, Journal of Applied Physics 64 (12), R93 (1988).

[Die07] W. Diehl, P. Brick, B. Kunert, S. Reinhard, K. Volz, and W. Stolz, Low threshold 1260 nm (GaIn) (NAs) semiconductor disk laser, Applied Physics Letters 91 (7), 071103 (2007), ISSN 00036951. 
[Eis99] H. Eisele, O. Flebbe, T. Kalka, C. Preinesberger, F. Heinrichsdorff, A. Krost, D. Bimberg, and M. Dähne-Prietsch, Cross-sectional scanning-tunneling microscopy of stacked InAs quantum dots, Applied Physics Letters 75 (1), 106 (1999)

[Eke91] M. J. Ekenstedt, S. M. Wang, and T. G. Andersson, Temperature-dependent critical layer thickness for $I n_{0.36} G a_{0.64} A s / G a A s$ single quantum wells, Applied Physics Letters 58 (8), 854 (1991).

[Fan06] L. Fan, M. Fallahi, J. Hader, A. R. Zakharian, J. V. Moloney, J. T. Murray, R. Bedford, W. Stolz, and S. W. Koch, Multichip vertical-external-cavity surfaceemitting lasers: a coherent power scaling scheme, Opt. Lett. 31 (24), 3612 (2006)

[Fan07a] L. Fan, M. Fallahi, A. Zakharian, J. Hader, J. Moloney, R. Bedford, J. Murray, W. Stolz, and S. Koch, Extended Tunability in a Two-Chip VECSEL, Photonics Technology Letters, IEEE 19 (8), 544 (2007), ISSN 1041-1135.

[Fan07b] L. Fan, C. Hessenius, M. Fallahi, J. Hader, H. Li, J. V. Moloney, W. Stolz, S. W. Koch, J. T. Murray, and R. Bedford, Highly strained InGaAs/GaAs multiwatt vertical-external-cavity surface-emitting laser emitting around 1170 nm, Applied Physics Letters 91 (13), 131114 (2007), ISSN 00036951.

[Feu08] M. Feucker, R. Seguin, S. Rodt, A. Hoffmann, and D. Bimberg, Decay dynamics of neutral and charged excitonic complexes in single InAs/GaAs quantum dots, Applied Physics Letters 92 (6), 063116 (2008), ISSN 00036951.

[Fu03] L. Fu, P. Lever, H. H. Tan, C. Jagadish, P. Reece, and M. Gal, Suppression of interdiffusion in InGaAs/GaAs quantum dots using dielectric layer of titanium dioxide, Applied Physics Letters 82 (16), 2613 (2003)

[Fuj99] K. Fujii, M. Satoh, K. Kawamura, and H. Gotoh, Dependence of carbon incorporation on growth conditions for unintentionally doped AlGaAs during metalorganic vapor-phase epitaxy, Journal of Crystal Growth 204 (1-2), 10 (1999), ISSN 0022-0248.

[Ger03] E. Gerster, I. Ecker, S. Lorch, C. Hahn, S. Menzel, and P. Unger, Orangeemitting frequency-doubled GaAsSb/GaAs semiconductor disk laser, Journal of Applied Physics 94 (12), 7397 (2003), ISSN 00218979.

[Ger05] T. D. Germann, A. Strittmatter, T. Kettler, K. Posilovic, K. Schatke, K. Pötschke, D. Feise, U. W. Pohl, and D. Bimberg, Entwicklung der Oberflächenmorpholo- 
gie von InGaAs/GaAs - Quantenpunktstrukturen, III/V Semiconductor Epitaxy Workshop (2005).

[Ger07] T. Germann, A. Strittmatter, T. Kettler, K. Posilovic, U. Pohl, and D. Bimberg, MOCVD of InGaAs/GaAs quantum dots for lasers emitting close to $1.3 \mu \mathrm{m}$, Journal of Crystal Growth 298, 591 (2007).

[Ger08a] T. Germann, A. Strittmatter, U. Pohl, D. Bimberg, J. Rautiainen, M. Guina, and O. Okhotnikov, Quantum-dot semiconductor disk lasers, Journal of Crystal Growth 310 (23), 5182 (2008), ISSN 0022-0248, as proceedings of the 14th ICMOVPE conference.

[Ger08b] T. D. Germann, A. Strittmatter, J. Pohl, U. W. Pohl, D. Bimberg, J. Rautiainen, M. Guina, and O. G. Okhotnikov, High-power semiconductor disk laser based on InAs/GaAs submonolayer quantum dots, Applied Physics Letters 92 (10), $101123(2008)$

[Ger08c] T. D. Germann, A. Strittmatter, J. Pohl, U. W. Pohl, D. Bimberg, J. Rautiainen, M. Guina, and O. G. Okhotnikov, Temperature-stable operation of a quantum dot semiconductor disk laser, Applied Physics Letters 93 (5), 051104 (2008), ISSN 00036951.

[Ger10] T. D. Germann, A. Strittmatter, A. Mutig, a. M. Nadtochiy, J. a. Lott, S. a. Blokhin, L. Y. Karachinsky, V. a. Shchukin, N. N. Ledentsov, U. W. Pohl, and D. Bimberg, Monolithic electro-optically modulated vertical cavity surface emitting laser with $10 \mathrm{~Gb} / \mathrm{s}$ open-eye operation, Physica Status Solidi (C) 7 (10), 2552 (2010), ISSN 18626351.

[Ger12] T. D. Germann, W. Hofmann, A. M. Nadtochiy, J.-H. Schulze, A. Mutig, A. Strittmatter, and D. Bimberg, Electro-optical resonance modulation of vertical-cavity surface-emitting lasers, Opt. Express 20 (5), 5099 (2012).

[Gon04] Q. Gong, P. Offermans, R. Nötzel, P. M. Koenraad, and J. H. Wolter, Capping process of InAs/GaAs quantum dots studied by cross-sectional scanning tunneling microscopy, Applied Physics Letters 85 (23), 5697 (2004).

[Got04] V. Gottschalch, G. Leibiger, and D. Spemann, X-ray investigations of the intrinsic carbon-incorporation during the MOVPE growth of AlxGa1-xAs, Zeitschrift für anorganische und allgemeine Chemie 630 (10), 1419 (2004), ISSN 00442313.

[Gou59] G. Gould, The LASER, light amplification by stimulated emission of radiation, The Ann Arbor Conference on Optical Pumping (1959). 
[Gra97] N. Grandjean, J. Massies, and O. Tottereau, Surface segregation in (Ga,In)As/GaAs quantum boxes, Phys. Rev. B 55 (16), R10189 (1997).

[Gra05] D. Granados and J. M. García, Determination of the energy levels on InAs quantum dots with respect to the GaAs conduction band, Nanotechnology 16 (5), S282 (2005).

[Gra10] K. Gradkowski, T. C. Sadler, L. O. Mereni, V. Dimastrodonato, P. J. Parbrook, G. Huyet, and E. Pelucchi, Crystal defect topography of Stranski-Krastanow quantum dots by atomic force microscopy, Applied Physics Letters 97 (19), $191106(2010)$

[Gru02] M. Grundmann (editor), Nano-Optoelectronics, Springer, Berlin Heidelberg (2002).

[Gös81] U. Gösele and F. Morehead, Diffusion of zinc in gallium arsenide: A new model, Journal of Applied Physics 52 (7), 4617 (1981), ISSN 00218979.

[Gui06a] D. Guimard, M. Nishioka, S. Tsukamoto, and Y. Arakawa, High density InAs/GaAs quantum dots with enhanced photoluminescence intensity using antimony surfactant-mediated metal organic chemical vapor deposition, Applied Physics Letters 89 (18), 183124 (2006)

[Gui06b] D. Guimard, S. Tsukamoto, M. Nishioka, and Y. Arakawa, $1.55 \mu$ m emission from InAs/GaAs quantum dots grown by metal organic chemical vapor deposition via antimony incorporation, Applied Physics Letters 89 (8), 083116 (2006)

[Gui07] D. Guimard, Y. Arakawa, M. Ishida, S. Tsukamoto, M. Nishioka, Y. Nakata, H. Sudo, T. Yamamoto, and M. Sugawara, Ground state lasing at $1.34 \mathrm{mu}$ $m$ from InAs/GaAs quantum dots grown by antimony-mediated metal organic chemical vapor deposition, Applied Physics Letters 90 (24), 241110 (2007).

[Gui08] D. Guimard, M. Ishida, N. Hatori, Y. Nakata, H. Sudo, T. Yamamoto, M. Sugawara, and Y. Arakawa, CW Lasing at $1.35 \mu \mathrm{m}$ From Ten InAs-Sb:GaAs Quantum-Dot Layers Grown by Metal-Organic Chemical Vapor Deposition, Photonics Technology Letters, IEEE 20 (10), 827 (2008), ISSN 1041-1135.

[Gui09] D. Guimard, M. Ishida, L. Li, M. Nishioka, Y. Tanaka, H. Sudo, T. Yamamoto, H. Kondo, M. Sugawara, and Y. Arakawa, Interface properties of InAs quantum dots produced by antimony surfactant-mediated growth: Etching of segregated antimony and its impact on the photoluminescence and lasing characteristics, Applied Physics Letters 94 (10), 103116 (2009) 
[Hai02] V. A. Haisler, F. Hopfer, R. L. Sellin, A. Lochmann, K. Fleischer, N. Esser, W. Richter, N. N. Ledentsov, D. Bimberg, C. Möller, and N. Grote, MicroRaman studies of vertical-cavity surface-emitting lasers with $\mathrm{Al}_{x} \mathrm{O}_{y} / \mathrm{GaAs}$ distributed Bragg reflectors, Applied Physics Letters 81 (14), 2544 (2002).

[Han04a] M. Hanke, D. Grigoriev, M. Schmidbauer, P. Schäfer, R. Köhler, U. W. Pohl, R. L. Sellin, D. Bimberg, N. D. Zakharov, and P. Werner, Diffuse X-ray scattering of InGaAs/GaAs quantum dots, Physica E 21 (2-4), 684 (2004).

[Han04b] M. Hanke, D. Grigoriev, M. Schmidbauer, P. Schäfer, R. Köhler, R. L. Sellin, U. W. Pohl, and D. Bimberg, Vertical composition gradient in InGaAs/GaAs alloy quantum dots as revealed by high-resolution $x$-ray diffraction, Applied Physics Letters 85 (15), 3062 (2004).

[Har98] A. Hartmann, C. Dieker, M. Hollfelder, H. Hardtdegen, A. Förster, and H. Lüth, Spontaneous formation of a tilted AlGaAs/GaAs superlattice during AlGaAs growth, Applied Surface Science 123-124, 704 (1998), ISSN 0169-4332, proceedings of the Sixth International Conference on the Formation of Semiconductor Interfaces.

[Has03] J. Hastie, J.-M. Hopkins, C. Jeon, S. Calvez, D. Burns, M. Dawson, R. Abram, E. Riis, A. Ferguson, W. Alford, T. Raymond, and A. Allerman, Microchip vertical external cavity surface emitting lasers, Electronics Letters 39 (18), 1324 (2003), ISSN 0013-5194.

[HCC75] J. H. C. Casey, D. D. Sell, and K. W. Wecht, Concentration dependence of the absorption coefficient for $n$ - and $p$ - type GaAs between 1.3 and $1.6 \mathrm{eV}$, Journal of Applied Physics 46 (1), 250 (1975).

[Hei96] F. Heinrichsdorff, A. Krost, M. Grundmann, D. Bimberg, A. Kosogov, and P. Werner, Self-organization processes of InGaAs/GaAs quantum dots grown by metalorganic chemical vapor deposition, Applied Physics Letters 68 (23), 3284 (1996), ISSN 00036951.

[Hei97a] F. Heinrichsdorff, Self organization phenomena of InGaAs/GaAs quantum dots grown by metalorganic chemical vapour deposition, Journal of Crystal Growth 170 (1-4), 568 (1997), ISSN 00220248.

[Hei97b] F. Heinrichsdorff, M.-H. Mao, N. Kirstaedter, A. Krost, D. Bimberg, A. O. Kosogov, and P. Werner, Room-temperature continuous-wave lasing from stacked InAs/GaAs quantum dots grown by metalorganic chemical vapor deposition, Applied Physics Letters 71 (1), 22 (1997) 
[Hei97c] R. Heitz, M. Veit, N. Ledentsov, A. Hoffmann, D. Bimberg, V. Ustinov, P. Kop'ev, and Z. Alferov, Energy relaxation by multiphonon processes in InAs/GaAs quantum dots, Physical Review B 56 (16), 10435 (1997), ISSN 0163-1829.

[Hei98a] F. Heinrichsdorff, MOCVD growth and laser applications of In( Ga)As/GaAs Quantum Dots, Dissertation, Technische Universität Berlin (1998).

[Hei98b] F. Heinrichsdorff, M. Grundmann, O. Stier, A. Krost, and D. Bimberg, Influence of In/Ga intermixing on the optical properties of InGaAs/GaAs quantum dots, Journal of Crystal Growth 195 (1-4), 540 (1998).

[Hei02] H. Heidemeyer, S. Kiravittaya, C. Muller, N. Y. Jin-Phillipp, and O. G. Schmidt, Closely stacked InAs/GaAs quantum dots grown at low growth rate, Applied Physics Letters 80 (9), 1544 (2002)

[Her95] R. Herrick, Y. Cheng, P. Petroff, M. Hibbs-Brenner, and R. Morgan, Spectrallyfiltered electroluminescence of vertical-cavity surface-emitting lasers, Photonics Technology Letters, IEEE 7 (10), 1107 (1995), ISSN 1041-1135.

[Hir94] H. Hirayama, K. Matsunaga, M. Asada, and Y. Suematsu, Lasing action of Ga0.67In0.33As/GaInAsP/InP tensile-strained quantum-box laser, Electronics Letters 30 (2), 142 (1994), ISSN 00135194.

[Hoo74] A. Hooper, B. Tuck, and A. Baker, Diffusion of zinc in indium phosphide at $700 \hat{A}^{\circ} \mathrm{C}$, Solid-State Electronics 17 (6), 531 (1974), ISSN 00381101.

[Hop04] J.-M. Hopkins, S. A. Smith, C. W. Jeon, H. D. Sun, D. Burns, S. Calvez, M. D. Dawson, T. Jouhti, and M. Pessa, 0.6 W CW GaInNAs vertical external-cavity surface emitting laser operating at $1.32 \mu \mathrm{m}$, Electronics Letters 40 (1), 30 (2004), ISSN 00135194.

[Hop07] F. Hopfer, A. Mutig, G. Fiol, M. Kuntz, V. Shchukin, V. Haisler, T. Warming, E. Stock, S. Mikhrin, I. Krestnikov, D. Livshits, A. Kovsh, C. Bornholdt,

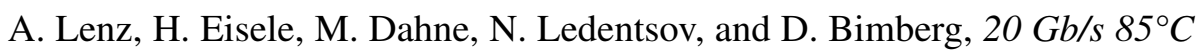
Error-Free Operation of VCSELs Based on Submonolayer Deposition of Quantum Dots, Selected Topics in Quantum Electronics, IEEE Journal of 13 (5), 1302 (2007), ISSN 1077-260X.

[Hou86] Y.-M. Houng and T. S. Low, Te doping of GaAs and AlxGal-xAs using diethyltellurium in low pressure OMVPE, Journal of Crystal Growth 77 (1-3), 272 (1986) 
[How05] P. Howe, E. C. L. Ru, E. Clarke, R. Murray, and T. S. Jones, Quantification of segregation and strain effects in InAs/GaAs quantum dot growth, Journal of Applied Physics 98 (11), 113511 (2005)

[Här01] R. Häring, Miniature Pulsed Laser Sources: Repetition Rates from Kilohertz to Gigahertz, Dissertation, Swiss Federal Institute of Technology Zürich (2001).

[Här06] A. Härkönen, S. Suomalainen, E. Saarinen, L. Orsila, R. Koskinen, O. Okhotnikov, S. Calvez, and M. Dawson, 4 W single-transverse mode VECSEL utilising intra-cavity diamond heat spreader, Electronics Letters 42 (12), 693 (2006), ISSN 0013-5194.

[Här07] A. Härkönen, J. Rautiainen, M. Guina, J. Konttinen, P. Tuomisto, L. Orsila, M. Pessa, and O. G. Okhotnikov, High power frequency doubled GaInNAs semiconductor disk laser emitting at 615 nm, Opt. Express 15 (6), 3224 (2007)

[Hsu92] W.-C. Hsu, S.-Z. Chang, and W. Lin, A Study of Layer Thickness and Interface Qualities of Strained $\operatorname{In}_{x} G a_{1-x} A s /$ GaAs Layers, Jpn. J. Appl. Phys. 31 (1), 26 (1992).

[Huf98] D. L. Huffaker, G. Park, Z. Zou, O. B. Shchekin, and D. G. Deppe, $1.3 \mu \mathrm{m}$ room-temperature GaAs-based quantum-dot laser, Applied Physics Letters 73 (18), 2564 (1998), ISSN 00036951.

[Ino06] J. Inoue, T. Isu, K. Akahane, and M. Tsuchiya, Saturable absorption of highly stacked InAs quantum dot layer in $1.5 \mathrm{mu}$ m band, Applied Physics Letters 89 (15), 151117 (2006)

[Iwa84] T. Iwamoto, K. Mori, M. Mizuta, and H. Kukimoto, Doped InGaP grown by MOVPE on GaAs, Journal of Crystal Growth 68 (1), 27 (1984), ISSN 00220248.

[Jac07] M. Jacquemet, N. Picqué, G. Guelachvili, A. Garnache, I. Sagnes, M. Strassner, and C. Symonds, Continuous-wave $1.55 \mu \mathrm{m}$ diode-pumped surface emitting semiconductor laser for broadband multiplex spectroscopy, Opt. Lett. 32 (11), 1387 (2007).

[Kai04] I. N. Kaiander, R. L. Sellin, T. Kettler, N. N. Ledentsov, D. Bimberg, N. D. Zakharov, and P. Werner, $1.24 \mu \mathrm{m}$ InGaAs/GaAs quantum dot laser grown by metalorganic chemical vapor deposition using tertiarybutylarsine, Applied Physics Letters 84 (16), 2992 (2004), ISSN 00036951. 
[Kai06] I. Kaiander, MOCVD growth of InGaAs/GaAs QDs for long wavelength lasers and VCSELs, Dissertation, Technische Universität Berlin (2006).

[Kak96] H. Kakinuma, M. Mohri, and M. Akiyama, Characterization of Oxygen and Carbon in Undoped AlGaAs Grown by Organometallic Vapor-Phase Epitaxy, Jpn. J. Appl. Phys. 36 (1A), 23 (1996).

[Kan94] Y. Kaneko and K. Kishino, Refractive indices measurement of $(G a \operatorname{InP})_{m} /(A l \operatorname{InP})_{n}$ quasi-quaternaries and GaInP/AlInP multiple quantum wells, Journal of Applied Physics 76 (3), 1809 (1994), ISSN 00218979.

[Ket10] T. Kettler, High Brightness Semiconductor Lasers, Dissertation, Technische Universität Berlin (2010)

[Khr97] O. M. Khreis, W. P. Gillin, and K. P. Homewood, Interdiffusion: A probe of vacancy diffusion in III-V materials, Phys. Rev. B 55 (23), 15813 (1997).

[Kim04] S. Kim, Y. Wang, M. Keever, and J. Harris, High-frequency modulation characteristics of $1.3 \mu \mathrm{m}$ InGaAs quantum dot lasers, IEEE Photonics Technology Letters 16 (2), 377 (2004)

[Kim06] K. Kim, J. Yoo, S. Lee, S. Lim, J. Kim, J. Lee, S. Cho, T. Kim, and Y. Park, Highly efficient InGaAs $Q W$ vertical external cavity surface emitting lasers emitting at $1060 \mathrm{~nm}$, Journal of Crystal Growth 287 (2), 629 (2006), ISSN 0022-0248, the 16th American Conference on Crystal Growth and Epitaxy ACCGE 16.

[Kim07] K.-S. Kim, J. Yoo, G. Kim, S. Lee, S. Cho, J. Kim, T. Kim, and Y. Park, 920-nm Vertical-External-Cavity Surface-Emitting Lasers With a Slope Efficiency of 58\% at Room Temperature, IEEE Photonics Technology Letters 19 (20), 1655 (2007), ISSN 1041-1135.

[Kir94] N. Kirstaedter, N. Ledentsov, M. Grundmann, V. Bimberg, D. andUstinov, S. Ruvimov, M. Maximov, P. Kop'ev, Z. Alferov, U. Richter, P. Werner, U. Gösele, and J. Heydenreich, Low threshold, large $T_{0}$ injection laser emission from (InGa)As quantumdots, El. Lett. 30 (17), 1416 (1994).

[Kli07] C. F. Klingshirn, Semiconductor Optics, Springer, $3^{r} d$ edition (2007), ISBN 978-3-540-38345-1.

[Klo08] P. Klopp, F. Saas, M. Zorn, M. Weyers, and U. Griebner, 290-fs pulses from a semiconductor disk laser, Opt. Express 16 (8), 5770 (2008) 
[Kon07] J. Konttinen, A. Härkönen, P. Tuomisto, M. Guina, J. Rautiainen, M. Pessa, and O. Okhotnikov, High-power $(>1 \mathrm{~W})$ dilute nitride semiconductor disk laser emitting at $1240 \mathrm{~nm}$, New Journal of Physics 9 (5), 140 (2007), ISSN 1367-2630.

[Kre01] I. Krestnikov, N. Ledentsov, A. Hoffmann, and D. Bimberg, Arrays of TwoDimensional Islands Formed by Submonolayer Insertions: Growth, Properties, Devices, physica status solidi (a) 183 (2), 207 (2001), ISSN 0031-8965.

[Kro63] H. Kroemer, A proposed class of hetero-junction injection lasers, Proceedings of the IEEE 51 (12), 1782 (1963), ISSN 0018-9219.

[Kro96a] A. Krost, G. Bauer, and J. Woitok, High resolution $x$-ray diffraction, in G. Bauer and W. Richter (editors), Optical Characterization of Epitaxial Semiconductor Layers, Springer (1996), ISBN 3-540-59129-X.

[Kro96b] A. Krost, F. Heinrichsdorff, D. Bimberg, A. Darhuber, and G. Bauer, Highresolution x-ray diffraction of self-organized InGaAs/GaAs quantum dot structures, Applied Physics Letters 68 (6), 785 (1996).

[Kum11] N. Kumagai, S. Ohkouchi, M. Shirane, Y. Igarashi, M. Nomura, Y. Ota, S. Yorozu, S. Iwamoto, and Y. Arakawa, Effects of growth temperature of partial GaAs cap on InAs quantum dots in In-flush process for single dot spectroscopy, Physica Status Solidi (C) 8 (2), 248 (2011), ISSN 18626351.

[Kuz99] M. Kuznetsov, F. Hakimi, R. Sprague, and A. Mooradian, Design and Characteristics of High-Power (>0.5-W CW) Diode-Pumped Vertical-External-Cavity Surface-Emitting Semiconductor Lasers with Circular TEM 00 Beams, IEEE J. of Selected Topics in Quantum Electronics 5, 561 (1999).

[Lag07] A. A. Lagatsky, F. M. Bain, C. T. A. Brown, W. Sibbett, D. A. Livshits, G. Erbert, and E. U. Rafailov, Low-loss quantum-dot-based saturable absorber for efficient femtosecond pulse generation, Applied Physics Letters 91 (23), 231111 (2007).

[Lan80] R. Lang and K. Kobayashi, External optical feedback effects on semiconductor injection laser properties, Quantum Electronics, IEEE Journal of 16 (3), 347 (1980), ISSN 0018-9197.

[Led94] N. N. Ledentsov, V. M. Ustinov, A. Y. Egorov, M. V. Zhukov, A. E.and Maximov, I. G. Tabatadze, and P. S. Kop'ev, Optical properties of heterostructures with InGaAs-GaAs quantum clusters, Fiz. i Tekh. Poluprovodn. 28, 1484 (1994). 
[Led07] N. N. Ledentsov, D. Bimberg, F. Hopfer, A. Mutig, V. a. Shchukin, A. V. Savel'ev, G. Fiol, E. Stock, H. Eisele, M. Dähne, D. Gerthsen, U. Fischer, D. Litvinov, A. Rosenauer, S. S. Mikhrin, a. R. Kovsh, N. D. Zakharov, and P. Werner, Submonolayer Quantum Dots for High Speed Surface Emitting Lasers, Nanoscale Research Letters 2 (9), 417 (2007), ISSN 1931-7573.

[Lee88] Y. H. Lee, J. L. Jewell, S. J. Walker, C. W. Tu, J. P. Harbison, and L. T. Florez, Electrodispersive multiple quantum well modulator, Applied Physics Letters 53 (18), 1684 (1988)

[Lee95] J. W. Lee, S. J. Pearton, C. R. Abernathy, W. S. Hobson, F. Ren, and C. S. Wu, Investigation of wet etching solutions for In0.5Ga0.5P, Solid-State Electronics 38 (11), 1871 (1995), ISSN 0038-1101.

[Lee00] P. Leerungnawarat, H. Cho, D. Hays, J. Lee, M. Devre, B. Reelfs, D. Johnson, J. Sasserath, C. Abernathy, and S. Pearton, Selective dry etching of InGaP over GaAs in inductively coupled plasmas, Journal of Electronic Materials 29, 586 (2000), ISSN 0361-5235, 10.1007/s11664-000-0049-9.

[Lee06] J. Lee, S. Lee, T. Kim, and Y. Park, 7 W high-efficiency continuous-wave green light generation by intracavity frequency doubling of an end-pumped vertical external-cavity surface emitting semiconductor laser, Applied Physics Letters 89 (24), 241107 (2006).

[Lem04] A. Lemaître, G. Patriarche, and F. Glas, Composition profiling of InAs/GaAs quantum dots, Applied Physics Letters 85 (17), 3717 (2004)

[Len90] G. Lengyel, K. Jelley, and R. Engelmann, A semi-empirical model for electroabsorption in GaAs/AlGaAs multiple quantum well modulator structures, IEEE Journal of Quantum Electronics 26 (2), 296 (1990), ISSN 00189197.

[Len02] A. Lenz, R. Timm, H. Eisele, C. Hennig, R. L. Becker, S. K. Sellin, U. W. Pohl, D. Bimberg, and M. Dähne, Reversed truncated cone composition distribution of $\mathrm{In}_{0.8} \mathrm{Ga} a_{0.2}$ As quantumdots overgrown by an $\operatorname{In}_{0.1} G a_{0.9}$ As layer in a $\mathrm{GaAs}$ matrix, Appl. Phys. Lett. 81 (27), 5150 (2002).

[Len11] A. Lenz, H. Eisele, J. Becker, J.-H. Schulze, T. D. Germann, F. Luckert, K. Pötschke, E. Lenz, L. Ivanova, A. Strittmatter, D. Bimberg, U. W. Pohl, and M. Dähne, Atomic structure and optical properties of InAs submonolayer depositions in GaAs, Journal of Vacuum Science \& Technology B 29 (4), 04D104 (2011), ISSN 10711023. 
[Leo94] D. Leonard, K. Pond, and P. Petroff, Critical layer thickness for self-assembled InAs islands on GaAs, Physical Review B 50 (16), 11687 (1994), ISSN 0163 1829.

[Leo96] R. Leon, Y. Kim, C. Jagadish, M. Gal, J. Zou, and D. J. H. Cockayne, Effects of interdiffusion on the luminescence of InGaAs/GaAs quantum dots, Applied Physics Letters 69 (13), 1888 (1996), ISSN 00036951.

[Leo98] R. Leon, C. Lobo, J. Zou, T. Romeo, and D. Cockayne, Stable and Metastable InGaAs/GaAs Island Shapes and Surfactantlike Suppression of the Wetting Transformation, Physical Review Letters 81 (12), 2486 (1998), ISSN 00319007.

[Les99] L. F. Lester, A. Stintz, H. Li, T. C. Newell, E. A. Pease, B. A. Fuchs, and K. J. Malloy, Optical characteristics of $1.24 \mu \mathrm{m}$ InAs quantum-dot laser diodes, IEEE PHOTONICS TECHNOLOGY LETTERS 11 (8), 931 (1999), ISSN 1041-1135.

[Leu98] S. Leu, F. Höhnsdorf, W. Stolz, R. Becker, A. Salzmann, and A. Greiling, $C$ - and $O$-incorporation in (AlGa)As epitaxial layers grown by MOVPE using TBAs, Journal of Crystal Growth 195 (1-4), 98 (1998).

[Liu04] H. Y. Liu, I. R. Sellers, M. Gutiérrez, K. M. Groom, W. M. Soong, M. Hopkinson, J. P. R. David, R. Beanland, T. J. Badcock, D. J. Mowbray, and M. S. Skolnick, Influences of the spacer layer growth temperature on multilayer InAs/GaAs quantum dot structures, Journal of Applied Physics 96 (4), 1988 (2004).

[Lot00] J. Lott, N. Ledentsov, V. Ustinov, N. Maleev, A. Zhukov, A. Kovsh, M. Maximov, B. Volovik, Z. Alferov, and D. Bimberg, InAs-InGaAs quantum dot VCSELs on GaAs substrates emitting at 1.3 [micro sign]m, Electronics Letters 36 (16), 1384 (2000), ISSN 00135194.

[Lot05] J. Lott, A. Kovsh, N. Ledentsov, and D. Bimberg, GaAs-Based InAs/InGaAs Quantum Dot Vertical Cavity and Vertical External Cavity Surface Emitting Lasers Emitting Near 1300 nm, Lasers and Electro-Optics, 2005. CLEO/Pacific Rim 2005. Pacific Rim Conference on p. 160 - 161 (2005)

[Lum87] R. M. Lum, J. K. Klingert, and M. G. Lamont, Use of tertiarybutylarsine in the metalorganic chemical vapor deposition growth of GaAs, Applied Physics Letters 50 (5), 284 (1987). 
[Mai60] T. H. Maiman, Stimulated Optical Radiation in Ruby, Nature 187 (4736), 493 (1960), ISSN 0028-0836.

[Mak96] T. Makino, Analytical formulas for the optical gain of quantum wells, IEEE Journal of Quantum Electronics 32 (3), 493 (1996), ISSN 00189197.

[Mal88] R. J. Malik, R. N. Nottenberg, E. F. Schubert, J. F. Walker, and R. W. Ryan, Carbon doping in molecular beam epitaxy of GaAs from a heated graphite filament, Applied Physics Letters 53 (26), 2661 (1988), ISSN 00036951.

[Ma197] S. Malik, C. Roberts, R. Murray, and M. Pate, Tuning self-assembled InAs quantum dots by rapid thermal annealing, Applied Physics Letters 71 (14), 1987 (1997).

[Mas83] W. T. Masselink, Y.-C. Chang, and H. Morkoç, Binding energies of acceptors in GaAs - $A l_{x} G a_{1-x} A s$ quantum wells, Physical Review B 28 (12), 7373 (1983), ISSN 0163-1829.

[Mas95] M. Mashita, H. Ishikawa, and T. Izumiya, Comparative study on carbon incorporation in MOCVD AlGaAs layers between arsine and tertiarybutylarsine, Journal of Crystal Growth 155 (3-4), 164 (1995), ISSN 0022-0248.

[Max00] M. V. Maximov, A. F. Tsatsul'nikov, B. V. Volovik, D. S. Sizov, Y. M. Shernyakov, I. N. Kaiander, A. E. Zhukov, A. R. Kovsh, S. S. Mikhrin, V. M. Ustinov, Z. I. Alferov, R. Heitz, V. A. Shchukin, N. N. Ledentsov, D. Bimberg, Y. G. Musikhin, and W. Neumann, Tuning quantum dot properties by activated phase separation of an InGa(Al)As alloy grown on InAs stressors, Phys. Rev. B 62 (24), 16671 (2000).

[Maz96] A. Mazuelas, H. Nörenberg, R. Hey, and H. T. Grahn, Growth and x-ray characterization of strain compensated GaAs/AlAs distributed Bragg reflectors, Applied Physics Letters 68 (6), 806 (1996), ISSN 00036951.

[Mc'75] J. A. Mc'Hugh, Secondary Ion Mass Spectroscopy, in A. W. Czanderna (editor), Methods of Surface Analysis, Elsevier (1975).

[Mil84] D. A. B. Miller, D. S. Chemla, T. C. Damen, A. C. Gossard, W. Wiegmann, T. H. Wood, and C. A. Burrus, Band-Edge Electroabsorption in Quantum Well Structures: The Quantum-Confined Stark Effect, Phys. Rev. Lett. 53 (22), 2173 (1984).

[Mil85] D. A. B. Miller, D. S. Chemla, T. C. Damen, A. C. Gossard, W. Wiegmann, T. H. Wood, and C. A. Burrus, Electric field dependence of optical absorption near the band gap of quantum-well structures, Phys. Rev. B 32 (2), 1043 (1985) 
[Mol98] N. Moll, M. Scheffler, and E. Pehlke, Influence of surface stress on the equilibrium shape of strained quantum dots, Physical Review B 58 (8), 4566 (1998), ISSN 0163-1829.

[Mon81] B. Monemar, Degradation Processes in Semiconductor Lasers, Physica Scripta 24 (2), 367 (1981), ISSN 0031-8949.

[Muk94] K. Mukai, N. Ohtsuka, M. Sugawara, and S. Yamazaki, Self-Formed $\mathrm{In}_{0.5} \mathrm{Ga} a_{0.5}$ As Quantum Dots on GaAs Substrates Emitting at $1.3 \mu \mathrm{m}$, Jpn. J. Appl. Phys. 33 (12A), L1710 (1994).

[Muk99] K. Mukai, Y. Nakata, K. Otsubo, M. Sugawara, N. Yokoyama, and H. Ishikawa, 1.3- $\mu m$ CW lasing of InGaAs-GaAs quantum dots at room temperature with a threshold current of $8 \mathrm{~mA}$, IEEE Photonics Technology Letters 11 (10), 1205 (1999), ISSN 10411135.

[Mun89] S. Munnix, R. K. Bauer, D. Bimberg, J. J. S. Harris, R. Köhrbrück, E. C. Larkins, C. Maierhofer, D. E. Mars, and J. N. Miller, Growth kinetics, impurity incorporation, defect generation, and interface quality of molecular-beam epitaxy grown AlGaAs/GaAs quantum wells: Role of group III and group V fluxes, J. Vac. Sci. Technol. B 7 (4), 704 (1989).

[Nun05] N. Nuntawong, S. Huang, Y. B. Jiang, C. P. Hains, and D. L. Huffaker, Defect dissolution in strain-compensated stacked InAs/GaAs quantum dots grown by metalorganic chemical vapor deposition, Applied Physics Letters 87 (11), 113105 (2005)

[Nun07] N. Nuntawong, J. Tatebayashi, P. S. Wong, and D. L. Huffaker, Localized strain reduction in strain-compensated InAs/GaAs stacked quantum dot structures, Applied Physics Letters 90 (16), 163121 (2007)

[Osh94] J. Oshinowo, M. Nishioka, S. Ishida, and Y. Arakawa, Highly uniform InGaAs/GaAs quantum dots ( $15 \mathrm{~nm}$ ) by metalorganic chemical vapor deposition, Applied Physics Letters 65 (11), 1421 (1994)

[Ou92] Z. Y. Ou, S. F. Pereira, E. S. Polzik, and H. J. Kimble, $85 \%$ efficiency for $\mathrm{cw}$ frequency doubling from 1.08 to 0.54 , $\mu m$, Opt. Lett. 17 (9), 640 (1992).

[Pa197] E. D. Palik (editor), Handbook of Optical Constants of Solids, Academic Press (1997), ISBN 978-0-12-544415-6.

[Par00] G. Park, O. Shchekin, D. Huffaker, and D. Deppe, Low-threshold oxide-confined 1.3- $\mu$ m quantum-dot laser, IEEE Photonics Technology Letters 12 (3), 230 (2000), ISSN 10411135. 
[Par04] S.-K. Park, J. Tatebayashi, and Y. Arakawa, Formation of ultrahigh-density InAs/AlAs quantum dots by metalorganic chemical vapor deposition, Applied Physics Letters 84 (11), 1877 (2004), ISSN 00036951.

[Pas94] R. Paschotta, P. Kürz, R. Henking, S. Schiller, and J. Mlynek, 82\% Efficient continuous-wave frequency doubling of $1.06 \mu \mathrm{m}$ with a monolithic $\mathrm{MgO}: \mathrm{LiNbO3}$ resonator, Opt. Lett. 19 (17), 1325 (1994)

[Pas04] A. Passaseo, V. Tasco, M. D. Giorgi, M. T. Todaro, M. D. Vittorio, and R. Cingolani, Long wavelength emission in $\operatorname{In}_{x} G a_{1-x}$ As quantum dot structures grown in a GaAs barrier by metalorganic chemical vapor deposition, Applied Physics Letters 84 (11), 1868 (2004)

[Pat99] A. Patanè, A. Polimeni, P. C. Main, M. Henini, and L. Eaves, High-temperature light emission from InAs quantum dots, Applied Physics Letters 75 (6), 814 (1999), ISSN 00036951.

[Poh08a] J. Pohl, MOCVD-Wachstum von Quantenpunkt-basierten Oberflächenemittern, Diploma thesis, Technische Universität Berlin (2008).

[Poh08b] U. W. Pohl, S. Rodt, and A. Hoffmann, Optical properties of III-V Quantum Dots, in D. Bimberg (editor), Semiconductor Nanostructures, Springer (2008), ISBN 978-3-540-77898-1.

[Po199] A. Polimeni, A. Patanè, M. Henini, L. Eaves, and P. Main, Temperature dependence of the optical properties of InAs/AlyGa1-yAs self-organized quantum dots, Physical Review B 59 (7), 5064 (1999), ISSN 0163-1829.

[Pöt04] K. Pötschke, L. Müller-Kirsch, R. Heitz, R. L. Sellin, U. W. Pohl, D. Bimberg, N. Zakharov, and P. Werner, Ripening of self-organized InAs quantum dots, Physica E: Low-dimensional Systems and Nanostructures 21 (2-4), 606 (2004), ISSN 13869477.

[Qia88] G.-X. Qian, R. Martin, and D. Chadi, First-principles study of the atomic reconstructions and energies of Ga- and As-stabilized GaAs(100) surfaces, Physical Review B 38 (11), 7649 (1988), ISSN 0163-1829.

[Rau08] J. Rautiainen, J. Lyytikäinen, A. Sirbu, A. Mereuta, A. Caliman, E. Kapon, and O. G. Okhotnikov, $2.6 \mathrm{~W}$ optically-pumped semiconductor disk laser operating at 1.57- $\mu$ m using wafer fusion, Optics Express 16 (26), 21881 (2008), ISSN 1094-4087. 
[Rau10] J. Rautiainen, I. Krestnikov, M. Butkus, E. U. Rafailov, and O. G. Okhotnikov, Optically pumped semiconductor quantum dot disk laser operating at $1180 \mathrm{~nm}$., Optics letters 35 (5), 694 (2010), ISSN 1539-4794.

[Rib02] C. Ribbat, Hochleistungs-Quantenpunkt-Halbleiterlaser, Dissertation, Technische Universität Berlin (2002)

[Rus03] S. A. Rushworth, L. M. Smith, M. S. Ravetz, K. M. Coward, R. Odedra, R. Kanjolia, S. W. Bland, F. Dimroth, and A. W. Bett, Correlation of reduced oxygen content in precursors with improved MOVPE layer quality, Journal of Crystal Growth 248, 86 (2003), ISSN 0022-0248.

[Rut06] A. Rutz, V. Liverini, D. Maas, B. Rudin, A.-R. Bellancourt, S. Schon, and U. Keller, Passively modelocked GaInNAs VECSEL at centre wavelength around $1.3 \mu \mathrm{m}$, Electronics Letters 42 (16), 926 (2006), ISSN 0013-5194.

[Ryu95] S.-W. Ryu, I. Kim, B.-D. Choe, and W. G. Jeong, The effect of strain on the interdiffusion in InGaAs/GaAs quantum wells, Applied Physics Letters 67 (10), 1417 (1995)

[San99] S. Sanguinetti, M. Henini, M. Grassi Alessi, M. Capizzi, P. Frigeri, and S. Franchi, Carrier thermal escape and retrapping in self-assembled quantum dots, Physical Review B 60 (11), 8276 (1999), ISSN 0163-1829.

[Sas07] H. Sasakura, S. Kayamori, S. Adachi, and S. Muto, Effect of indium-flush method on the control of photoluminescence energy of highly uniform selfassembled InAs quantum dots by slow molecular beam epitaxy growth, Journal of Applied Physics 102 (1), 013515 (2007), ISSN 00218979.

[Sch58] A. Schawlow and C. Townes, Infrared and Optical Masers, Physical Review 112 (6), 1940 (1958), ISSN 0031-899X.

[Sch07] A. Schliwa, M. Winkelnkemper, and D. Bimberg, Impact of size, shape, and composition on piezoelectric effects and electronic properties of In $(G a) A s / G a A s$ quantum dots, Phys. Rev. B 76 (20), 205324 (2007).

[Sch08] P. Schlosser, S. Calvez, J. E. Hastie, S. Jin, T. D. Germann, A. Strittmatter, U. W. Pohl, D. Bimberg, and M. D. Dawson, Characterisation of an InAs quantum dot semiconductor disk laser, in Conference on Lasers and Electro-Optics, $\mathrm{p}$. CWD5, Optical Society of America (2008), ISBN 978-1-55752-859-9.

[Seg06] R. Seguin, A. Schliwa, T. D. Germann, S. Rodt, K. Pötschke, A. Strittmatter, U. W. Pohl, D. Bimberg, M. Winkelnkemper, T. Hammerschmidt, and P. Kratzer, 
Control of fine-structure splitting and excitonic binding energies in selected individual InAs/GaAs quantum dots, Applied Physics Letters 89 (26), 263109 (2006), ISSN 00036951.

[Sel00] R. Sellin, F. Heinrichsdorff, C. Ribbat, M. Grundmann, U. W. Pohl, and D. Bimberg, Surface flattening during MOCVD of thin GaAs layers covering InGaAs quantum dots, Journal of Crystal Growth 221 (1-4), 581 (2000), ISSN 00220248 .

[Shc95] V. A. Shchukin, N. N. Ledentsov, P. S. Kop'ev, and D. Bimberg, Spontaneous Ordering of Arrays of Coherent Strained Islands, Phys. Rev. Lett. 75 (16), 2968 (1995).

[Shc98a] V. Shchukin and D. Bimberg, Strain-driven self-organization of nanostructures on semiconductor surfaces, Applied Physics A: Materials Science \& Processing 67, 687 (1998), ISSN 0947-8396.

[Shc98b] V. Shchukin, D. Bimberg, V. Malyshkin, and N. Ledentsov, Vertical correlations and anticorrelations in multisheet arrays of two-dimensional islands, Physical Review B 57 (19), 12262 (1998), ISSN 0163-1829.

[Shc99] V. Shchukin and D. Bimberg, Spontaneous ordering of nanostructures on crystal surfaces, Reviews of Modern Physics 71 (4), 1125 (1999), ISSN 0034-6861.

[Shc08] V. A. Shchukin, N. N. Ledentsov, J. A. Lott, H. Quast, F. Hopfer, L. Y. Karachinsky, M. Kuntz, P. Moser, A. Mutig, A. Strittmatter, V. P. Kalosha, and D. Bimberg, Ultra high-speed electro-optically modulated VCSELs: modeling and experimental results, SPIE Proceedings, Physics and Simulation of Optoelectronic Devices XVI 6889 (2008).

[Smi04] S. Smith, J. Hopkins, J. Hastie, D. Burns, S. Calvez, M. Dawson, T. Jouhti, J. Kontinnen, and M. Pessa, Diamond-microchip GaInNAs vertical externalcavity surface-emitting laser operating $C W$ at $1315 \mathrm{~nm}$, Electronics Letters 40 (15), 935 (2004), ISSN 00135194.

[Smo01] P. M. Smowton, E. Herrmann, Y. Ning, H. D. Summers, P. Blood, and M. Hopkinson, Optical mode loss and gain of multiple-layer quantum-dot lasers, Applied Physics Letters 78 (18), 2629 (2001).

[Son03] R. Songmuang, S. Kiravittaya, and O. Schmidt, Shape evolution of InAs quantum dots during overgrowth, Journal of Crystal Growth 249 (3-4), 416 (2003), ISSN 00220248 . 
[Ste97] E. Steimetz, F. Schienle, J. T. Zettler, and W. Richter, Stranski-Krastanov formation of InAs quantum dots monitored during growth by reflectance anisotropy spectroscopy and spectroscopic ellipsometry, Journal of Crystal Growth 170 (14), 208 (1997), ISSN 0022-0248.

[Str38] I. N. Stranski and L. Krastanow, Zur Theorie der orientierten Ausscheidung von Ionenkristallen aufeinander, Monatshefte für Chemie / Chemical Monthly 71, 351 (1938), ISSN 0026-9247.

[Str99] G. B. Stringfellow, Organometallic Vapor-Phase Epitaxy: Theory and Practice, Academic Press, London, second edition (1999).

[Str06] A. Strittmatter, T. D. Germann, T. Kettler, K. Posilovic, U. W. Pohl, and D. Bimberg, Alternative precursor metal-organic chemical vapor deposition of InGaAs/GaAs quantum dot laser diodes with ultralow threshold at $1.25 \mu \mathrm{m}$, Applied Physics Letters 88 (26), 262104 (2006).

[Str08a] A. Strittmatter, T. Germann, T. Kettler, K. Posilovic, J. Pohl, U. Pohl, and D. Bimberg, Suppression of the wavelength blue shift during overgrowth of InGaAs-based quantum dots, Journal of Crystal Growth 310 (23), 5066 (2008), ISSN 0022-0248, 14th ICMOVPE.

[Str08b] A. Strittmatter, T. Germann, J. Pohl, U. Pohl, D. Bimberg, J. Rautiainen, M. Guina, and O. Okhotnikov, $1040 \mathrm{~nm}$ vertical external cavity surface emitting laser based on inGaAs quantum dots grown in Stranski-Krastanow regime, Electronics Letters 44 (4), 290 (2008), ISSN 0013-5194.

[Sun91] S. Z. Sun, E. A. Armour, K. Zheng, and C. F. Schaus, Zinc and tellurium doping in GaAs and AlxGal-xAs grown by MOCVD, Journal of Crystal Growth 113 (1-2), 103 (1991), ISSN 0022-0248.

[Sun05] Y. Sun, S. F. Cheng, G. Chen, R. F. Hicks, J. G. Cederberg, and R. M. Biefeld, The effect of antimony in the growth of indium arsenide quantum dots in gallium arsenide (001), Journal of Applied Physics 97 (5), 053503 (2005)

[Suz77] T. Suzuki and M. Ogawa, Degradation of photoluminescence intensity caused by excitation-enhanced oxidation of GaAs surfaces, Applied Physics Letters 31 (7), 473 (1977), ISSN 00036951.

[Suz08] R. Suzuki, T. Miyamoto, T. Sengoku, and F. Koyama, Reduction of spacer layer thickness of InAs quantum dots using GaNAs strain compensation layer, Applied Physics Letters 92 (14), 141110 (2008) 
[Tak62] S. Takagi, Dynamical theory of diffraction applicable to crystals with any kind of small distortion, Acta Crystallographica 15 (12), 1311 (1962), ISSN 0365110X.

[Tak69] S. Takagi, A Dynamical Theory of Diffraction for a Distorted Crystal, Journal of the Physical Society of Japan 26 (5), 1239 (1969), ISSN 0031-9015.

[Tak99] T. Takamoto, M. Yumaguchi, E. Ikeda, T. Agui, H. Kurita, and M. Al-Jassim, Mechanism of $\mathrm{Zn}$ and Si diffusion from a highly doped tunnel junction for InGaP/GaAs tandem solar cells, Journal of Applied Physics 85 (3), 1481 (1999), ISSN 00218979.

[Tak11] K. Takada, Y. Tanaka, T. Matsumoto, M. Ekawa, H. Song, Y. Nakata, M. Yamaguchi, K. Nishi, T. Yamamoto, M. Sugawara, and Y. Arakawa, Widetemperature-range $10.3 \mathrm{Gbit} / \mathrm{s}$ operations of $1.3 \mu \mathrm{m}$ high-density quantum-dot DFB lasers, Electronics Letters 47 (3), 206 (2011), ISSN 0013-5194.

[Tan86] H. Tanaka, Y. Kawamura, and H. Asahi, Refractive indices of $I n_{0.49} G a_{0.51-x} A l_{x} P$ lattice matched to GaAs, Journal of Applied Physics 59 (3), 985 (1986), ISSN 00218979.

[Tan91] T. Y. Tan, U. Gösele, and S. Yu, Point defects, diffusion mechanisms, and superlattice disordering in gallium arsenide-based materials, Critical Reviews in Solid State and Materials Sciences 17 (1), 47 (1991)

[Tat01] J. Tatebayashi, M. Nishioka, and Y. Arakawa, Over 1.5 mm light emission from InAs quantum dots embedded in InGaAs strain-reducing layer grown by metalorganic chemical vapor deposition, Applied Physics Letters 78 (22), 3469 (2001).

[Tat04] J. Tatebayashi, Y. Arakawa, N. Hatori, H. Ebe, M. Sugawara, H. Sudo, and A. Kuramata, InAs/GaAs self-assembled quantum-dot lasers grown by metalorganic chemical vapor deposition-Effects of postgrowth annealing on stacked InAs quantum dots, Applied Physics Letters 85 (6), 1024 (2004).

[Tat05a] J. Tatebayashi, N. Hatori, M. Ishida, H. Ebe, M. Sugawara, Y. Arakawa, H. Sudo, and A. Kuramata, $1.28 \mu$ m lasing from stacked InAs/GaAs quantum dots with low-temperature-grown AlGaAs cladding layer by metalorganic chemical vapor deposition, Applied Physics Letters 86 (5), 053107 (2005).

[Tat05b] J. Tatebayashi, M. Ishida, N. Hatori, H. Ebe, H. Sudou, A. Kuramata, M. Sugawara, and Y. Arakawa, Lasing at $1.28 \mu \mathrm{m}$ of InAs-GaAs quantum dots with AlGaAs cladding layer grown by metal-organic chemical vapor deposition, Selected Topics in Quantum Electronics 11 (5), 1027 (2005), ISSN 1077-260X. 
[Tau64] D. Taupin, Théorie dynamique de la diffraction des rayons X par les cristaux, Bulletin de la Societé française de Minéralogie et de Cristallographie 87, 469 (1964).

[Tou07] J. Tourrenc, S. Bouchoule, A. Khadour, J. Decobert, A. Miard, J. Haimand, and $\mathrm{X}$. Oudar, High power single-longitudinal-mode OP-VECSEL at $1.55 \mathrm{~lm}$ with hybrid metal-metamorphic Bragg mirror, Electronics Letters 43 (14) (2007), ISSN 0013-5194.

[Tro04] A. C. Tropper, H. D. Foreman, A. Garnache, K. G. Wilcox, and S. H. Hoogland, Vertical-external-cavity semiconductor lasers, J. Phys. D 37, R75 (2004).

[Ust99] V. M. Ustinov, N. A. Maleev, A. E. Zhukov, A. R. Kovsh, A. Y. Egorov, A. V. Lunev, B. V. Volovik, I. L. Krestnikov, Y. G. Musikhin, N. A. Bert, P. S. Kop'ev, Z. I. Alferov, N. N. Ledentsov, and D. Bimberg, InAs/InGaAs quantum dot structures on GaAs substrates emitting at $1.3 \mu \mathrm{m}$, Applied Physics Letters 74 (19), 2815 (1999)

[vdP59] L. J. van der Pauw, A method of measuring the resistivity and hall coefficient on lamellae of arbitrary shape, Philips Technical Review 20 (8), 220 (1959).

[vE07] J. van Eisden, M. Yakimov, V. Tokranov, M. Varanasi, E. M. Mohammed, I. a. Young, and S. Oktyabrsky, Modulation properties of VCSEL with intracavity modulator, Proceedings of SPIE 6484, 64840A (2007), ISSN 0277786X.

[vE08] J. van Eisden, M. Yakimov, V. Tokranov, M. Varanasi, O. Rumyantsev, E. M. Mohammed, I. a. Young, and S. R. Oktyabrsky, High frequency resonance-free loss modulation in a duo-cavity VCSEL, Proceedings of SPIE 6908, 69080M (2008), ISSN 0277786X.

[Ven84] J. A. Venables, G. D. Spiller, and M. Hanbücken, Nucleation and Growth of Thin Films, Rep. Prog. Phys. 47, 399 (1984)

[vG88] G. J. van Gurp, D. L. A. Tjaden, G. M. Fontijn, and P. R. Boudewijn, Zinc diffusion in InGaAsP, Journal of Applied Physics 64 (7), 3468 (1988), ISSN 00218979 .

[vO83] A. H. van Ommen, Examination of models for Zn diffusion in GaAs, Journal of Applied Physics 54 (9), 5055 (1983).

[Vur01] I. Vurgaftman, J. R. Meyer, and L. R. Ram-Mohan, Band parameters for III-V compound semiconductors and their alloys, Journal of Applied Physics 89 (11), 5815 (2001), ISSN 00218979. 
[Wal01] T. Walther, A. Cullis, D. Norris, and M. Hopkinson, Nature of the StranskiKrastanow Transition during Epitaxy of InGaAs on GaAs, Physical Review Letters 86 (11), 2381 (2001), ISSN 0031-9007.

[Wan04] X.-D. Wang, N. Liu, C. K. Shih, S. Govindaraju, and J. A. L. Holmes, Spatial correlation-anticorrelation in strain-driven self-assembled InGaAs quantum dots, Applied Physics Letters 85 (8), 1356 (2004).

[Wes09] P. Westbergh, J. Gustavsson, A. Haglund, M. Skold, A. Joel, and A. Larsson, High-Speed, Low-Current-Density $850 \mathrm{~nm}$ VCSELs, IEEE Journal of Selected Topics in Quantum Electronics 15 (3), 694 (2009), ISSN 1077-260X.

[Wog97] U. Woggon, W. Langbein, J. M. Hvam, A. Rosenauer, T. Remmele, and D. Gerthsen, Electron microscopic and optical investigations of the indium distribution in GaAs capped $I n_{x} G a_{1-x}$ As islands, Applied Physics Letters 71 (3), 377 (1997)

[Xie95] Q. Xie, A. Madhukar, P. Chen, and N. P. Kobayashi, Vertically Self-Organized InAs Quantum Box Islands on GaAs(100), Phys. Rev. Lett. 75 (13), 2542 (1995).

[Xu96] Z. Xu, Z. Lu, X. Yang, Z. Yuan, B. Zheng, J. Xu, W. Ge, Y. Wang, J. Wang, and L. Chang, Carrier relaxation and thermal activation of localized excitons in self-organized InAs multilayers grown on GaAs substrates., Physical review. B, Condensed matter 54 (16), 11528 (1996), ISSN 0163-1829.

[Xu03a] Z. Xu, D. Birkedal, J. r. M. Hvam, Z. Zhao, Y. Liu, K. Yang, A. Kanjilal, and J. Sadowski, Structure and optical anisotropy of vertically correlated submonolayer InAs/GaAs quantum dots, Applied Physics Letters 82 (22), 3859 (2003). ISSN 00036951.

[Xu03b] Z. Xu, K. Leosson, D. Birkedal, V. Lyssenko, J. r. M. Hvam, and J. Sadowski, InGaAs/GaAs quantum-dot-quantum-well heterostructure formed by submonolayer deposition, Nanotechnology 14 (12), 1259 (2003), ISSN 0957-4484.

[Xu06] Z. Xu, Y. Zhang, J. M. Hvam, J. Xu, X. Chen, and W. Lu, Carrier dynamics in submonolayer InGaAs/GaAs quantum dots, Applied Physics Letters 89 (1), 013113 (2006), ISSN 00036951.

[Yan90] R. Yan, S. Corzine, L. Coldren, and I. Suemune, Corrections to the expression for gain in GaAs, IEEE Journal of Quantum Electronics 26 (2), 213 (1990), ISSN 00189197.

[Yan06] T. Yang, J. Tatebayashi, M. Nishioka, and Y. Arakawa, Improved surface morphology of stacked $1.3 \mathrm{mu} \mathrm{m}$ InAs/GaAs quantum dot active regions by introducing annealing processes, Applied Physics Letters 89 (8), 081902 (2006). 
[Yeh02] N.-T. Yeh, W.-S. Liu, S.-H. Chen, P.-C. Chiu, and J.-I. Chyi, InAs/GaAs quantum dot lasers with InGaP cladding layer grown by solid-source molecular-beam epitaxy, Applied Physics Letters 80 (4), 535 (2002).

[Yoo97] I. Yoon, B. Jeong, and H. Park, Zn diffusion of In0.5Ga0.5P investigated by photoluminescence measurements, Thin Solid Films 300 (1-2), 284 (1997), ISSN 00406090.

[Yu99] P. Yu, W. Langbein, K. Leosson, J. Hvam, N. Ledentsov, D. Bimberg, V. Ustinov, A. Egorov, A. Zhukov, A. Tsatsul'nikov, and Y. Musikhin, Optical anisotropy in vertically coupled quantum dots, Physical Review B 60 (24), 16680 (1999), ISSN 0163-1829.

[Zak05] A. Zakharian, J. Hader, J. Moloney, and S. Koch, VECSEL threshold and output power-shutoff dependence on the carrier recombination rates, IEEE Photonics Technology Letters 17 (12), 2511 (2005), ISSN 1041-1135. 


\section{Acknowledgments}

I am especially grateful to Prof. Dr. Dieter Bimberg who gave me the great opportunity to learn and work in his group on the exciting field of III-V epitaxy for nano-photonic devices, and who always supported the progress of my work.

My sincere gratitude is due to DR. ANDRÉ STRITTMATTER, the ideal partner for epitaxy, who contributed substantially to the achievements of this work, introduced me to epitaxy, and was always open for discussions.

Prof. DR. Udo PoHL was at all times open for discussions, knew always the right paper to cite, and provided many fruitful inputs into this work.

I am grateful to Prof. ShUn LiEn ChUANG, PhD for refereeing this thesis, and for many instructive discussions on the physics of nano-photonic devices.

Prof. DR. Michael Lehmann is acknowledged for presiding over my defense, and for providing outstanding TEM pictures.

I want to thank the whole epitaxial team for the excellent team play and say: Thanks a lot! Many thanks to KATHRIN SCHATKE for excellent support and assistance on all epitaxial problems, and JAN-HINDRIK SCHULZE for being a good friend and perfect colleague, for his outstanding efforts on the coding of the online lab book and for the great time as office roommate.

No lasers without processing (except for VECSEL ;-) many thanks for the fastest laser processing on earth to DR. THORSTEN KETTLER and KRISTIJAN POSILOVIC, and many thanks for processing of the EOM VCSEL to DR. AlEX MUTIG.

Thanks to DR. WERner HofmAnn and AleXey NADTOCHIY for characterization of the EOM VCSEL in order to reveal the intrinsic quality.

OLEG OKHOTNIKOV and his team in Tampere, Finland were great partners measuring all VECSEL devices.

Special thanks to the whole AG BIMBERG for being an outstanding team to work with.

Thank you TRACIE PENNIMPEDE, PHD for night long English proof-reading.

I am very grateful to my parents Rosemarie BARTHEL and HERBERT GERMANN for always helping and supporting me outside the physics world and many thanks to the very best brother JONATHAN GERMANN.

I want to thank my wonderful wife CONSTANZE NANDY for her love, and my lovely son EMIL MALO for being here. 